\title{
TITANIA: Model Free Interpretation of Residual Dipolar Couplings in the context of Organic Compounds
}

\author{
Felix A. Roth, Volker Schmidts, Christina M. Thiele* \\ Clemens-Schöpf-Institut für Organische Chemie und Biochemie, Technical University of Darmstadt, Alarich-Weiss-Str. 16, 64287 \\ Darmstadt (Germany), E-mail: cthiele@thielelab.de \\ NMR spectroscopy, configuration determination, residual dipolar couplings, model-free, vector orientation
}

\begin{abstract}
Residual dipolar couplings (RDCs) become increasingly important as additional NMR parameter in the structure elucidation of organic compounds, but are usually used in fitting procedures to discriminate between (computed) structures that are in accordance with RDCs and others that can be ruled out. Thus determination of configurations requires prior structural information. The direct use of RDCs as restraint to construct structures based on RDCs has only recently begun also in organic compounds. No protocol has been published though, which uses the vector and dynamics information available in multi alignment data sets directly for the joint determination of conformation and configuration of organic compounds. This is proposed within the current manuscript. We show that employing these data even a flat or random start structure converges into the correctly configured structure when employing multiple alignment data sets in our iterative procedure. The requirements in terms of number of RDCs and alignment media necessary are discussed in detail.
\end{abstract}

\section{INTRODUCTION}

Nuclear magnetic resonance spectroscopy (NMR) provides a wealth of spectroscopic observables encoding the molecular structure and dynamics of the investigated compounds. Complementary information is accessible from chemical shifts, isotropic scalar $J$ coupling constants and the nuclear Overhauser effect (nOe).1.4 Additionally, anisotropic observables of compounds aligned with respect to the magnetic field like residual dipolar couplings (RDCs) have proven to be a reliable tool for the determination ${ }^{5-10}$ and refinement ${ }^{11-15}$ of both small molecules and biomolecular structures. Standard protocols for RDC-based structure determination assume rigid structure models and a single tensor (e.g. the alignment tensor A) describing the molecular alignment following the classical formalism by Saupe. ${ }^{16-17}$ This tensor is generally unknown and calculated by a fitting procedure utilizing e.g. singular value decomposition (SVD) as introduced for RDC order matrix analyses by Losonczi in $1999 .{ }^{18}$ Discrimination of structure models is based on correlation of experimental RDCs $D_{\exp }$ and RDCs backcalculated from the order matrix model, $D_{\text {calc }}$ (see Figure 1). The description of conformational flexibility or other forms of intramolecular dynamics is often modeled by ensemble averaging of rigid equilibrium geometries ${ }^{10,19-20}$ thus limiting the information from experimental RDCs by the choice or parametrization of the models employed in fitting.

For the interpretation of RDCs in biomacromolecules, the so called "direct interpretation of dipolar couplings" (DIDC) $)^{21-22}$ and "model free approach" (MFA) $)^{23-24}$ have been proposed, which do not rely on a strict definition of an alignment tensor and a molecular geometry but rather directly express the experimental RDCs as a function of dynamically averaged vector orientations.
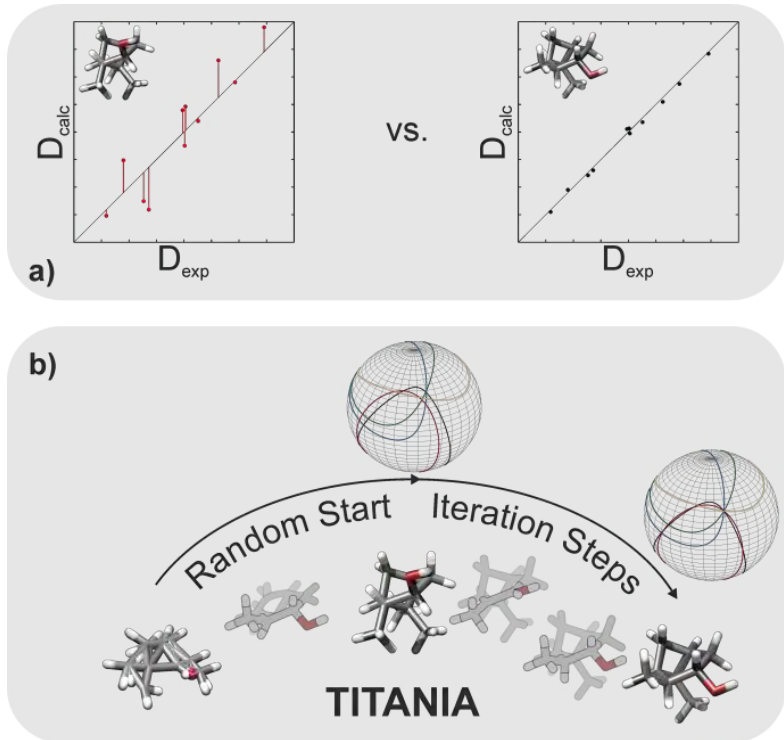

Figure 1. Conventional RDC analyses validate experimental data by correlating them with back calculated data (see top a). TITANIA directly interprets $\mathrm{RDC}$ data to derive $\mathrm{RDC}$ vector orientation and dynamic information to determine the correct stereochemistry of the compound (see bottom $\mathbf{b}$ ).

The DIDC minimizes the RDC vector motion needed to explain the experimental data while the MFA uses spherical harmonics, as proposed by Lipari and Szabo for the interpretation of relaxation data, ${ }^{25-26}$ to describe internal motion. The underlying algorithms were extended to iterative schemes to improve robustness and quality of results..$^{27-29}$ 
At the core of these methods is the requirement for at least five linearly independent sets of experimental RDCs (alignment conditions) and a large amount of RDC vectors, ${ }^{30}$ to simultaneously evaluate the corresponding relative vector orientations and their respective distribution(s). The wealth of RDC data experimentally accessible in labelled biomacromolecules enabled the development and broad application of these methods in that field. Modern developments in alignment media ${ }^{31-39}$ compatible with small organic molecules, introducing different alignment conditions combined with new NMR pulse sequences ${ }^{40-49}$ designed to give easier access to crucial long-range ${ }^{\mathrm{n}} D_{\mathrm{XH}}$ and ${ }^{\mathrm{n}} D_{\mathrm{HH}} \mathrm{RDCs}$ may now finally allow to extend the model free approach also to organic compounds. This is investigated within the current manuscript. The crucial linear independence of alignment conditions is assessed here by methods based on principle component analysis, like the self-consistency of dipolar couplings analysis (SECONDA) protocol by Hus and Brüschweiler. ${ }^{50-51}$

When applying the model free approach to small molecule RDC analysis, the additional challenge stems from the fact that the relative configuration of stereogenic centers is introduced as additional unknown, the determination of which is coupled to the determination of unknown conformation. Recent approaches for organic compounds focused on alternative algorithms employing (pseudo) force fields (FF). Cornilescu et al. introduced progressive stereo locking (PSL) in combination with FF methods in 2017 to resolve the relative configuration and conformation of small molecules in Xplor-NIH. ${ }^{52}$ In 2018 Immel et al. used a floating chirality restrained distance geometry and distance bound driven dynamics (fc-rDG/DDD) protocol to generate structural models and optimize them according to RDC restraints in configurational and conformational architect $\left(\mathrm{ConArch}{ }^{+}\right){ }^{53}$ Furthermore, tensorial restraints are employed in the MD protocol published as computer simulation of molecular structures (COSMOS) by Sternberg and Luy. ${ }^{54-56}$ These algorithms have in common that the optimization of the structures is performed on the difference of user defined experimental NMR observables and the back-calculated values to define or extend a (pseudo) force field. In addition, multiple alignment media are treated as individual sources of information for the refinement of structures and not globally as is proposed here.

We present a new five alignment media MFA, which has to the best of our knowledge not been established for organic compounds. It is implemented in the new $\mathrm{C}++$ program TITANIA (TITANIA performs iterative analysis of independent alignments), which interprets RDCs by directly calculating individual $R D C$ vector orientations and their dynamics. For the given sets of RDC data and an arbitrary starting geometry, this enables the combined investigation and refinement of conformation, relative configuration and internal dynamics from five of more RDC sets, which are interpreted as one global source of information on the $3 \mathrm{D}$ structure and the dynamical averaging of small molecules. We demonstrate the MFAbased RDC analysis with TITANIA on several small to mediumsized organic compounds with well-known stereochemistry and varying degrees of molecular complexity.
Theory. RDCs are anisotropic interactions described by the relation of inter-nuclear vector orientations with respect to the external magnetic field $B_{0}$ :

$$
D_{\mathrm{ij}}=\frac{\kappa_{\mathrm{ij}}}{\left\langle r_{\mathrm{ij}}^{3}\right\rangle} \frac{\left\langle 3 \cos ^{2} \Theta_{\mathrm{ij}}-1\right\rangle}{2}
$$

where $\kappa_{i j}=-\frac{\gamma_{i} \gamma_{j} \mu_{0} \hbar}{8 \pi^{2}}$ groups physical constants for two nuclei $i$ and $\mathrm{j}, r_{\mathrm{ij}}$ is the distance between the nuclei and $\Theta_{\mathrm{ij}}$ is the angle enclosed by the RDC vector and $B_{0}$. The \langle\rangle brackets denote time and ensemble averaging of the respective parameters. A common approximation neglects an explicit time average of the distances $r$ and instead uses their equilibrium distance.

Assuming a rigid geometry, the orientational order imposed onto the solute by the alignment medium is expressed by the Saupe order tensor $\mathbf{S}^{57-58}$ or its scaled analogue, the alignment tensor $\mathbf{A}^{17}$ The alignment tensor $\mathbf{A}$ is a traceless symmetric second-rank tensor, often expressed in terms of the Euler rotations $\boldsymbol{R}(\alpha, \beta, \gamma)$ necessary to rotate its principle axis system onto the arbitrary molecular frame and its axial $\left(A_{a}\right)$ and rhombic components $\left(A_{r}\right.$, or more generally, the rhombicity $R$ ) denoting its shape. This common property defined relative to an external reference is the reason for the global structure information contained in RDCs. Internal dynamics lead to deviations from this simple alignment model and have to be modeled appropriately (see below). In the classically used cross validation of structures with RDCs these dynamical aspects are described by multiple conformers. . $^{10,19-20,59-62}$

When dealing with rotations of tensors and inter nuclear vectors, as well as the overall molecular tumbling in the alignment medium, it is convenient to express equation 1 in terms of spherical harmonics: $:^{63}$

$$
\left\langle D_{\mathrm{k}}\right\rangle=A_{\mathrm{a}} D_{\max , \mathrm{k}} \sqrt{\frac{4 \pi}{5}}\left[\sqrt{\frac{3}{8} R\left(\left\langle Y_{2,2}\left(\theta_{\mathrm{k}}, \phi_{\mathrm{k}}\right)\right\rangle+\left\langle Y_{2,2}^{*}\left(\theta_{\mathrm{k}}, \phi_{\mathrm{k}}\right)\right\rangle\right)}\right]
$$

Here $D_{\max , \mathrm{k}}$ is the maximum possible dipolar coupling of the spin pair $\mathrm{k}$ (short for the nuclei $\mathrm{i}$ and $\mathrm{j}$ ), and $Y$ are the spherical harmonics of the spherical coordinates $\theta$ and $\phi$ (in the principle axis system (PAS) of A). Equivalent expressions can be formulated using Cartesian coordinates resulting in direction cosines. ${ }^{17-18}$ The time and ensemble averages in equation 2 may be resolved by utilizing Wigner rotations for the spherical harmonics, thus giving a mathematical separation of the overall molecular tumbling (alignment parameters) from the individual vector orientation (internal dynamics). ${ }^{59}$ 61

$$
\boldsymbol{R}(\alpha, \beta, \gamma) Y_{2, \mathrm{~m}}^{(2)}(\theta, \phi)=\sum_{\mathrm{M}=-2}^{2} e^{-\mathrm{i} \alpha \mathrm{M}} d_{\mathrm{M}, \mathrm{m}}^{(2)}(\beta) e^{-\mathrm{i} \gamma \mathrm{m}} Y_{2, \mathrm{M}}^{(2)}(\theta, \phi)
$$

Where $\boldsymbol{R}$ is a rotation applied to spherical harmonics, represented by the reduced Wigner elements $d_{M, m}^{(2)}$ using the Euler angles $\alpha$, $\beta$ and $\gamma$. This formalism simplifies the transformation into any arbitrary reference system. Common reference systems (see Figure 2 ) are the alignment frame (AF), the vector frame (VF) and arbitrary molecular frames (MF). The AF is the principle axis system of 
the alignment tensor where all non-diagonal elements become zero. In this frame, equation 2 is valid and no rotation has to be applied to describe the alignment. A more convenient frame when discussing molecular structure is the MF, which - barring any symmetryrelated arguments ${ }^{64}$ - can be chosen arbitrarily. By default, TITANIA uses the PAS of the inertia tensor of the input structure. The transformation between the MF and the AF is defined by the Euler angles $\alpha, \beta$ and $\gamma$ (see equation 3 ) obtained from the eigenvectors of $\mathbf{A}$. The additional VF, which is essential in the approach described herein, is unique for every $\mathrm{RDC}$ vector and describes the PAS of the individual vectors where $Y_{2, \mathrm{~m}}=0$ for $\mathrm{m} \neq 0$. The respective transformation between the VF and the MF is defined by the spherical coordinates $\theta$ and $\phi$. In principle a third rotation angle $\varphi$ can be defined, which describes the direction of the anisotropic $\mathrm{RDC}$ vector motion, i.e. the angle needed to align the major semiaxis with the $y$-axis of the anisotropic RDC vector cone (see red cone in Figure 2). This angle may be neglected if only RDC vector orientations are of interest (vide infra).

Starting from the individual VF of the respective RDC vectors a sequence of the described rotations ( $\mathrm{VF} \rightarrow \mathrm{MF} \rightarrow \mathrm{AF}$ ) can be used to obtain the general matrix equation 4 . The derivation of equation 4 is conducted as in the literature $\mathrm{e}^{23-24}$ (see supporting information).

$$
\mathbf{D}(M, K)=\langle\mathbf{F}\rangle(M, 5) \cdot\langle\mathbf{Y}\rangle(5, M)
$$

Here $\mathbf{D}$ contains $K$ RDCs in $M$ alignment conditions and is normalized to $A_{a}$ (row wise) and $D_{\max }$ (column wise). F combines the $M$ Wigner rotations and rhombicity $R$ of the respective alignment tensor and $\mathbf{Y}$ the spherical harmonics of the $K$ RDC vectors. This denotes the previously mentioned separation of internal (local) and external (global) motion(al averaging). The normalization of D fully removes external, alignment media dependent averaging and allows for a model free extraction of structural parameters, defined by the rotations between the MF and the VF, and internal dynamics, encoded in the magnitude of $Y_{2, \mathrm{~m}}$.
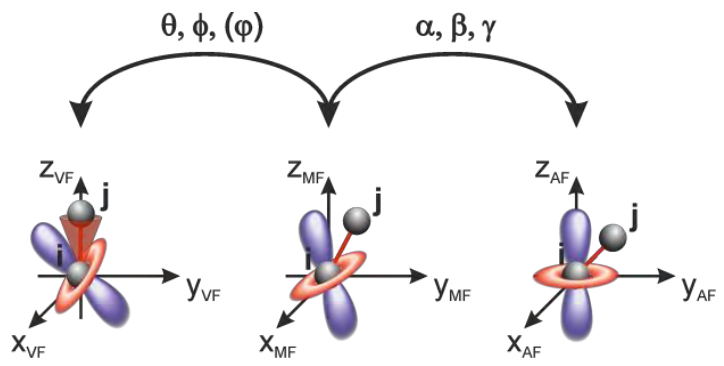

Figure 2. Reference frames used in the MFA for the determination of RDC vector orientations of the spin pair $i$ and $j$. The arbitrary molecular frame (MF, middle) can be transformed into either the vector frame (VF, left-hand side) or the alignment frame (AF, right hand side). The AF is defined as the PAS of $\boldsymbol{A}$ (represented by the surface plot of $Y_{2,0}$ with color encoding the sign) where all non-diagonal elements vanish. The respective rotation is defined by the Euler angles $\alpha, \beta$ and $\gamma$. In the VF the RDC vector is parallel to the z-axis and all spherical harmonics $Y_{2, \mathrm{~m}}$ become zero for $\mathrm{m} \neq 0$. The spherical coordinates $\theta$ and $\phi$ define the rotation between the VF and the MF. Dynamics of the RDC vector is represented by the red cone in the VF. The third angle $\varphi$, expressing the orientation of the anisotropic RDC motion, can be neglected when just describing the vector orientation.
With data from at least five independent alignment conditions, the spherical harmonics $\mathbf{Y}$ can be refined by SVD of $\mathbf{F}$. If additional alignment media are used for the optimization, the accuracy and precision can be improved, but no further structure information can be obtained. ${ }^{21}$ To extract the mean vector orientation from the respective spherical harmonics the transformation between MF and the respective VF has to be found (e.g. by the LevenbergMarquardt algorithm).

While there are recent approaches to simulate interactions of small molecules with alignment media and predict RDCs based on a combination of molecular dynamics simulations and potential based methods $s^{65-66}$ the individual alignment conditions of equation 4 are usually a priori unknown and have to be estimated before employing the MFA. ${ }^{23-24}$ To increase robustness of the MFA, Lakomek et al. proposed the self-consistent residual dipolar coupling based model-free analysis (SCRM), which essentially is the iterative implementation of the MFA to reduce a possible bias induced by the initial alignment tensor (and thereby the initial structure model). ${ }^{27}$ In this protocol, the initial MFA step estimates the starting alignment based on an initial model (e.g. a rigid X-ray structure, but as shown here any arbitrary set of coordinates is possible). The resulting $\theta$ and $\phi$ are used to start the iterative cycle, in which the refined spherical coordinates are used to recalculate the alignment tensors via SVD. The Wigner rotations are updated to refine the spherical harmonics and to finally update the spherical coordinates.

In addition to the structure parameters $\theta$ and $\phi$, the parameters $S_{R D C}^{2}$ and $\eta$ describing RDC dynamics, are calculated from the spherical harmonics, where $S_{R D C}^{2}$ is the order parameter for RDCs, following the definition of the Lipari-Szabo parameter $S_{L S}^{2} \cdot{ }^{25-26} S_{R D C}^{2}$ can be interpreted as the axial component of a local RDC tensor. The same is true for the anisotropy parameter $\eta$, which is directly linked to the third rotation angle $\varphi$ (see Figure 2).

For biomacromolecules, the challenge is often the large conformational space and distribution of internal dynamics sampled by RDCs. Small organic compounds on the other hand usually have fewer intrinsic degrees of freedom but the analysis of relative vector orientation from RDCs is complicated by the smaller number of RDCs available and the unknown relative configuration of stereogenic centers. The use of long-range RDCs needed for the MFA in combination with comparatively large structural changes of small organic compounds associated to these internal degrees of freedom may lead to instabilities of the optimization algorithm when updating the Wigner rotations. Thus TITANIA not only uses the refined spherical coordinates to recalculate the Wigner rotations but also performs a full structure optimization on every step for a superior estimation of the corresponding $D_{\max }$ values. The algorithm to update the structure and the interpretation of the dynamic information is discussed in the following sections.

\section{RESULTS AND DISCUSSION}

Interpretation of structural parameters. The spherical coordinates $\theta$ and $\varphi$ are the two parameters needed to define the directional vector of the respective spin pairs. One problem in RDC analysis is the indistinguishability of this vector and its inverse. 
A simple algorithm for updating structures would be to string the updated ${ }^{1} D_{\mathrm{ij}} \mathrm{RDC}$ vectors together, while keeping vectors constant that are not defined by RDCs (for more information on how this vector addition algorithm is implemented and on which flag needs to be used to activate it, see SI section 1.3.5). This approach has the downside that small errors in individual vector orientations can lead to major distortions in the overall structure due to propagation of errors. The differentiation of the two degenerate RDC vector directions, when updating the position of an atom i, is performed by assessing the agreement of long range $\mathrm{RDC}$ direction vectors $\left(\vec{r}_{\mathrm{li}, \mathrm{RDC}}\right)$ and the respective vectors in the updated structure model $\left(\vec{r}_{\mathrm{li}, \mathrm{struc}}\right)$ for the two possible vectors $\vec{r}_{\mathrm{ij}, \mathrm{RDC}}$ and $-\vec{r}_{\mathrm{ij}, \mathrm{RDC}}$. This assessment only affects the sign of the direction vector as determined by MFA and not the individual Cartesian coefficients.

A more advanced algorithm, implemented in the TITANIA workflow, uses redundant internal coordinates known from quantum chemical computing protocols. ${ }^{67-68}$ In this approach standard internal coordinates ${ }^{69}$, namely bond lengths, bond angles or dihedral angles of more atom tuples than needed to unambiguously define a structure are combined to form an overdetermined representation of a structure model. The holonomic terms (used synonymously for internal coordinates in the following) may easily be extended by additional parameters and subjected to optimization algorithms when calculating Cartesian coordinates. Obviously RDC direction vectors are a good choice in this context, with additional parameters like exclusion distances ${ }^{70}$ or chiral volumes (especially for planar centers and moieties). ${ }^{71}$

With the Cartesian coordinates of a starting geometry and a set of redundant internal coordinates (including RDC-based restraints) as input, the Cartesian displacement associated to the (small) internal coordinate displacement is evaluated following the iterative protocol by Peng et al. ${ }^{67}$ (see supporting information for implementation details). The iterative implementation allows for the full utilization of long-range couplings (see Figure 3 ) to discriminate the two possible vectors $\vec{r}_{\mathrm{ij}, \mathrm{RDC}}$ (for green position) and $-\vec{r}_{\mathrm{ij}, \mathrm{RDC}}$ (for red position). This global treatment not only discriminates the previously indistinguishable vector orientations but also leads to the best-fit solution of all RDCs for one nucleus and may also optimize the Cartesian coefficients in the process.
As shown in Figure 3 the best-fit position of all holonomic terms (bond lengths and angles; these were not marked to keep the figure simple) and all RDC information (solid arrows: ${ }^{1} D_{\mathrm{CH}}$ and dotted arrows: $\left.{ }^{n} D_{\mathrm{HH}}\right)$ is calculated using redundant internal coordinates. To achieve lower inversion barriers (i.e. to more easily sample the full conformational and configurational space), dihedral angle restraints were not included in the implemented algorithm.

Redundant internal coordinates in combination with sufficient experimental data should thus enable determination of the conformation and relative configuration of small organic compounds. As with all other approaches, absolute configurations cannot be derived by this algorithm. ${ }^{72}$ The two major downsides of this algorithm are the poor robustness of the algorithm towards large changes in internal coordinates and the lack of scalability towards larger compounds with increased degrees of freedom. The first point can lead to instabilities if large changes occur (e.g. due to large errors). This can be bypassed by downscaling the changes (see supporting information for more details) or by choosing the non-default vector addition algorithm. The second point is due to the SVD applied in this algorithm, which slows down drastically when optimizing large molecules. Improvements to the underlying algorithm to boost performance are currently under investigation.

Treatment of dynamics. As described by Meiler et al. ${ }^{23}$ dynamics interpretation is conducted analogous to the Lipari-Szabo interpretation of nOe data: ${ }^{25-26}$

$$
S_{\mathrm{RDC}, \mathrm{k}}^{2}=\frac{4 \pi}{5} \sum_{\mathrm{M}=-2}^{2}\left\langle Y_{2, \mathrm{M}}\left(\theta_{\mathrm{k}}, \phi_{\mathrm{k}}\right)\right\rangle \cdot\left\langle Y_{2, \mathrm{M}}^{*}\left(\theta_{\mathrm{k}}, \phi_{\mathrm{k}}\right)\right\rangle
$$

$S_{\mathrm{RDC}}^{2}$ is the local order parameter describing the dynamics of each individual RDC. In biomacromolecular NMR this parameter is crucial for the assessment of conformational changes and the target of all multi alignment approaches. ${ }^{21,23-24,27-29,73}$ Here we are primarily interested in obtaining the correct conformation and configuration, but nevertheless need to correctly account for dynamics. In MFA analysis the alignment is derived from a rigid structural model while experimental RDCs are additionally scaled by internal motion. This reduces the effective alignment magnitude. Therefore the refined spherical harmonics obtained from equation 4 and $S_{\mathrm{RDC}}^{2}$ are both equally scaled by the same factor.
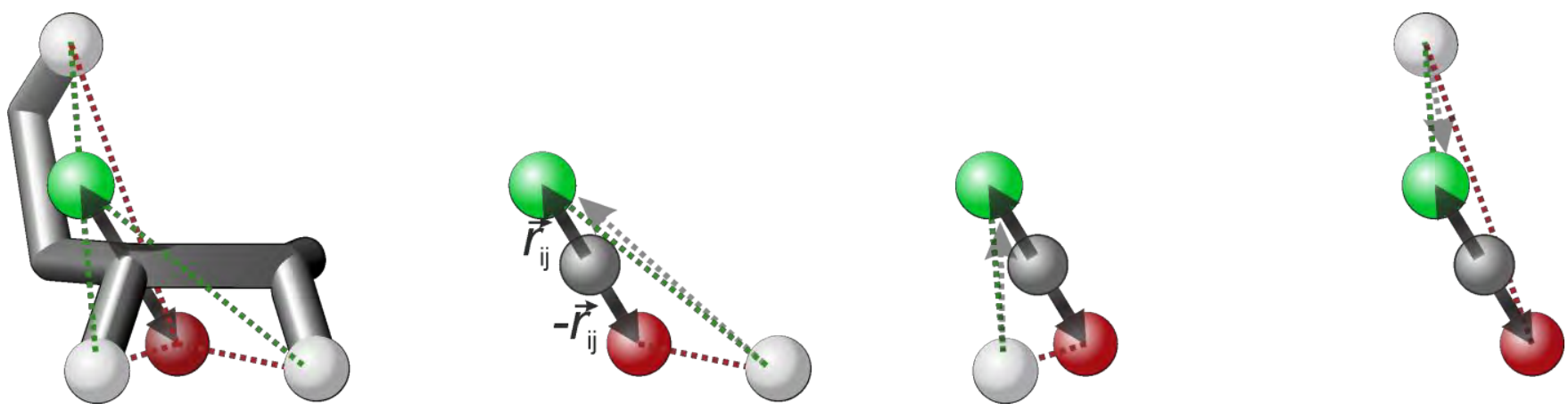

Figure 3. Representation of degenerate RDC vector orientations in the case of a ${ }^{1} D_{\mathrm{CH}}$ coupling (black vectors) resulting in two mathematically indistinguishable solutions (green and red atoms). When adding three additional ${ }^{\mathrm{n}} \mathrm{D}_{\mathrm{HH}}$ couplings (grey vectors in right hand part) the solution with the least mean error squares (green vs. red vectors) can be determined. Thereby the correct atom position can be determined. 
To remove this effect and extract the correct order parameter the scaling parameter $S_{\text {overall }}$ reflecting this reduction has to be estimat$\mathrm{ed}^{23}$ (see SI on how this is done within TITANIA).

Use of SECONDA to investigate linear independence. As described earlier the linear independence of the alignment media is crucial for a successful optimization by RDCs, especially when experimental errors are present. The simplest method to compare two alignments in the generalized angle $\beta$ as defined by Sass et al. ${ }^{74}$ This measure is useful for the pairwise comparison of two alignment conditions, e.g. when assessing enantiodifferentiation in chiral alignment media. ${ }^{75}$ When extending this to a large number of RDC sets the interpretation becomes difficult and is only possible qualitatively (see SI Figure S-3). Ideally one would want to analyze a data matrix containing all RDC sets (or information on those) at once. Tolman used the condition number of the RDC matrix obtained by SVD. ${ }^{21}$ A related but more adaptable ${ }^{76}$ method as proposed here is the principle component analysis (PCA) ${ }^{77-78}$ of the covariance matrix $\mathbf{C}$. The goal is to find a low dimensional pattern in a data matrix of $K$ RDCs measured in $M$ alignment media, to assess an underlying model - in our case five linearly independent alignment conditions. The PCA of $\mathbf{C}$ was implemented by Hus et al. in the SECONDA protocol to investigate the influence of structural noise (computed using a MD trajectory) on RDCs. ${ }^{50}$ The normalized covariance matrix of the RDCs (equation 6) is calculated and diagonalized to obtain its principle variances (eigenvalues $\lambda_{q}$ ) and eigenmodes (eigenvectors $|q\rangle$ ). Here, the principle variances indicate the amount of data variance that is captured by the respective dimension. In addition, the eigenmodes form the reference frame that captures the principle variances along their axes.

$$
C_{i j}=\frac{1}{M-1} \sum_{m}^{M} w_{m}\left(D_{i}^{m}-\left\langle D_{i}\right\rangle\right)\left(D_{j}^{m}-\left\langle D_{j}\right\rangle\right)
$$

The weighting $w_{m}$ of the individual RDC sets can be defined in different ways. TITANIA's standard implementation is reported in the SI (see equation 1). Closer inspection of the principle variances and their corresponding eigenmodes is required to disentangle the individual contributions to the overall RDC dataset. The distribution of the first five eigenvalues is a measure of the individual sampling of the dimensions. If five non-zero eigenvalues are obtained and these have similar contributions to the cumulative sum (see equation 7) this confirms linear independence of the five data sets. Additional eigenvalues will occur (dimensionality $>5$ ) if the RDCs contain heterogeneous behavior, like experimental error or structural noise.

The corresponding eigenvalues are evaluated either by the $\lambda_{5} / \lambda_{6}$ ratio (defined as $\rho_{5 / 6}$ gap by Hus and Brüschweiler) or their percentage of the total variance. The contribution of the RDC pairs to the individual principle variances, can be assessed in a local fashion, using the eigenvector components, or by using the global collectivity $\kappa_{\mathrm{q}}$ (see equation 8 ), which quantifies the contribution of the RDC pairs to the corresponding principle variance in percent (with the range $\left.\kappa_{\mathrm{q}}=[100 / \mathrm{K}, 100] \%\right){ }^{50,79}$

$$
S_{\mathrm{c}}\left(\lambda_{\mathrm{k}}\right)=\frac{\sum_{\mathrm{i}=1}^{\mathrm{k}} \lambda_{\mathrm{i}}}{\sum_{\mathrm{i}=1}^{K} \lambda_{\mathrm{i}}} \cdot 100 \%
$$

$$
\left.\left.\kappa_{\mathrm{q}}=\left.\left.\frac{1}{K} \exp \left[-\sum_{k=1}^{K}|| q\right\rangle_{\mathrm{k}}\right|^{2} \log (|| q\rangle_{\mathrm{k}}\right|^{2}\right)\right] \cdot 100 \%
$$

This allows the representation of an eigenvalue and eigenvector pair by the two values $\lambda_{q}$ and $\kappa_{q}$. The overall heterogeneity of individual RDC pairs can be expressed by the cumulative sum of the heterogeneous modes $a^{2}:{ }^{51}$

$$
a_{\mathrm{k}}^{2}=\sum_{\mathrm{q}=6}^{K} \lambda_{\mathrm{q}}\left|q_{\mathrm{k}}\right\rangle^{2}
$$

To the best of our knowledge there is no comprehensive application of SECONDA to the often limited RDC datasets available for organic compounds. Some examples for SECONDA analyses are given below, while a more extensive discussion on the interpretation of SECONDA runs is given in the supporting information.

The TITANIA protocol. The mathematical framework described herein is combined and extended in the TITANIA C++ software. A general flow scheme representing the major blocks of TITANIA is shown Figure 4.

TITANIA is a command line program that is started with a user defined input file containing RDCs, an initial structure model (coordinates and connectivity) and keywords to adapt the behavior of the optimization scheme. Examples for input files can be found in the supplementary material.

For a proper run a parametrization is performed, in which scaled and normalized RDC matrices are calculated, the structure parameters (holonomic terms) and redundant internal coordinates are set up. Additionally a molecular frame is defined by transforming the initial structure to the PAS of the inertia tensor. Using the in-house implementation of the SECONDA protocol the (normalized) RDC data matrix is assessed.

From there on the iterative refinement of the RDC and structure parameters starts. The orientation parameters of all RDC sets are calculated simultaneously using the SVD of the normalized cosine matrix $\mathbf{B}$ (see SI equations $2 \mathrm{a} / \mathrm{c}$ ). ${ }^{18,21}$ The alignment tensors obtained are used to build the Wigner rotations and update the spherical harmonics via SVD of the $\mathbf{F}$ matrix in equations 4. It is important to note that the spherical coordinates, and thereby the $\mathrm{RDC}$ direction vectors, are derived from the entirety of RDCs (see SI equation 17) and not by consideration of single datasets as individual sources of information. The spherical coordinates are used to construct the Cartesian coordinates based on redundant internal coordinates (see SI section 1.3.4). To estimate the uncertainty of the calculated parameters and check for convergence of the procedure, the refined structure models are used for a MonteCarlo bootstrap based on the user estimated experimental errors.

Application on Isopinocampheol. We have chosen Isopinocampheol (IPC) $\mathbf{1}$ as a test case as it is a bicyclic compound with a highly rigid scaffold, containing four chiral centers (C1, C2, C3, $\mathrm{C} 5$, with $\mathrm{C} 1$ and $\mathrm{C} 5$ being interdependent) and three pro-chiral centers ( $\mathrm{C} 4, \mathrm{C} 6$ and $\mathrm{C} 7)$. The rigidity allows for a separation of the configurational analysis from any conformational investigations. Furthermore plenty of RDC data are available, thus we assume that enough independent orientations are available (see below). 


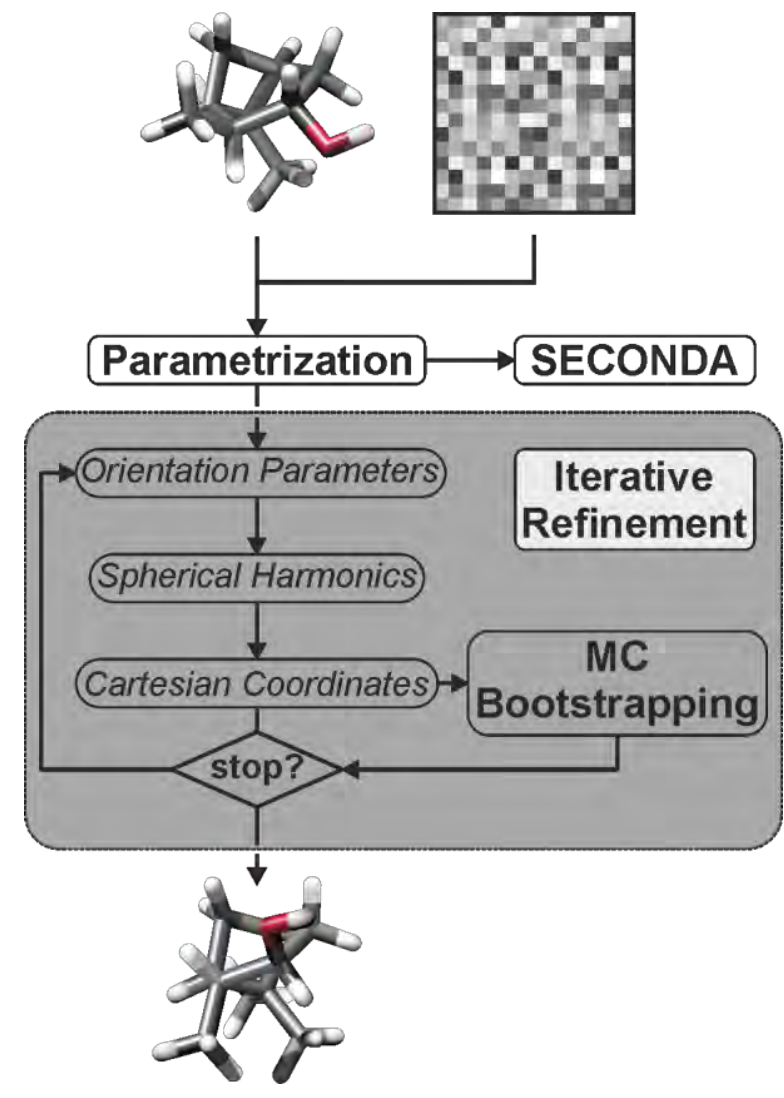

Figure 4. Schematic flowchart of the TITANIA protocol. The user has to define an initial input structure and RDCs from at least five alignment media (indicated as heatmap). TITANIA starts a parametrization of these data and performs SECONDA. The iterative refinement of the structure starts by updating the orientational parameters and calculating the refined spherical harmonics from this. Using redundant internal coordinates the updated Cartesian coordinates are calculated and a Monte-Carlo bootstrap is performed to check for convergence. The final output contains the updated mean structure and additional RDC information like $S_{\mathrm{RDC}}^{2}$, the SECONDA results and statistical information on the RDC vectors.

Several scenarios will be investigated: 20 artificial orientations for maximum sampling of the orientational space (setup 1-A), real orientation which are expected to be largely independent (setup 1B) and real orientations, which are chosen in a way to maximize linear dependence (setup 1-C). Additionally error was added to these data sets (setups 1-D to 1-F, see experimental section for more details). The amount of data is varied for the setups resulting in different runs with the number indicating the number of RDCs used (for a table which RDCs were used in which run, see SI Table S-81).

First we will discuss the optimization runs 1-A which employ 20 artificial RDC sets. Since linear independence is crucial for reliable optimizations, this will be investigated first by applying SECONDA to each RDC run (see Figure 5). Only the runs with 11, 23 and 39 RDCs per set are shown here. The respective data for all runs can be found in the supporting information (see section 2.2.1).
All plots show a similar trend. In the absence of heterogeneities like structural noise or experimental error on the RDCs, no more than five eigenvalues $\left(\lambda_{1-5}\right)$ differ from zero. In the presence of heterogeneities additional non-zero eigenvalues $\left(\lambda_{n}\right.$ with $\left.n \geq 6\right)$ would be expected. As data sets are artificial no experimental error is present and a rigid compound is investigated there are no heterogeneities. The ratio of the largest $\left(\lambda_{1}\right)$ and the lowest eigenvalue $\left(\lambda_{5}\right)$ gives a direct assessment of the sampling of independent orientations. In all runs for 1-A excellent ratios $\lambda_{1} / \lambda_{5}$ of $<10$ are found, indicating sufficient sampling of orientations.

When evaluating the cumulative sum $S_{\mathrm{c}}$ (see equation 7), the 1A examples all show a contribution of the smallest variance $\left(\lambda_{5}\right)$ to the overall variance of at least $6 \%$. This indicates that the five alignment media are independent. If linear dependence would be present, the contribution of the last eigenvalues to the overall variance would be much smaller (see run 1-C).

The associated collectivities $\kappa_{q}$ quantify if the variation of the $\mathrm{K}$ RDCs occurs in a more local or rather global fashion. When analyzing the $\kappa_{\mathrm{q}}$ values of Figure 5 it becomes clear, that the distribution of the collectivities becomes wider when adding ${ }^{\mathrm{n}} D_{\mathrm{HH}} \mathrm{RDCs}$ (1A23, see SI for further SECONDA plots: 1-A17 to 1-A39), while the overall pattern remains similar.

Based on the SECONDA analysis the 20 RDC sets of setup 1-A should form a solid basis for the iterative optimization using TITANIA which is conducted next. As a first test the C3-epimer was chosen. The chiral volumes are monitored to visualize the course of the optimization (see Figure 6). It is expected that the correct stereochemistry is reliably achieved for all centers, if sufficient data is used. If this condition is not met (see 1-A11 which uses only ${ }^{1} D_{\mathrm{CH}} \mathrm{RDC}$ ) the inverse $\mathrm{RDC}$ vector can be a possible result (see Figure 3 ). Adding even a few ${ }^{\mathrm{n}} \mathrm{D}_{\mathrm{HH}} \mathrm{RDC}$ improves the situation significantly, such that the correct relative configuration is obtained straight away (shown here for 23 RDCs, 1-A23). It works already with 17 RDCs (see 1-A17, Figure S-12 in the SI).

All runs show an inversion in the sign of the chiral volume of $\mathrm{C} 3$ in the course of the optimization (blue lines in Figure 6). In the case of 1-A11 (only ${ }^{1} D_{\mathrm{CH}}$ data) this inversion occurs instantaneously but remains correct only for the first 10 steps. The final structure contains the wrong configuration at $\mathrm{C} 3$. Still the correct orientation information on the $\mathrm{RDC}$ vector $\mathrm{C} 3-\mathrm{H} 3$ is obtained (for a similar finding in tubocurarine, see Figure 12). This is a direct consequence of the mathematically indistinguishable solutions discussed above and can be improved by inclusion of long-range RDCs. A hint on the inverse vector orientation is the distortion of the respective tetrahedral geometry. After the initial steps, a nearly planar arrangement of the $\mathrm{C} 3-\mathrm{C} 2, \mathrm{C} 3-\mathrm{O} 3$ and $\mathrm{C} 3-\mathrm{C} 4$ vectors is obtained. Without additional restraints, the redundant internal coordinates retain the optimization (in this case) in the wrong minimum / configuration. This becomes even clearer when no redundant coordinates are used for the optimization (see SI section 1.3.5). To assess geometry violations of the resulting structures, TITANIA calculates the magnitude of the chiral volumes for all possible permutations of the bond vectors and determines the respective rmsd between those values. ${ }^{80}$ 


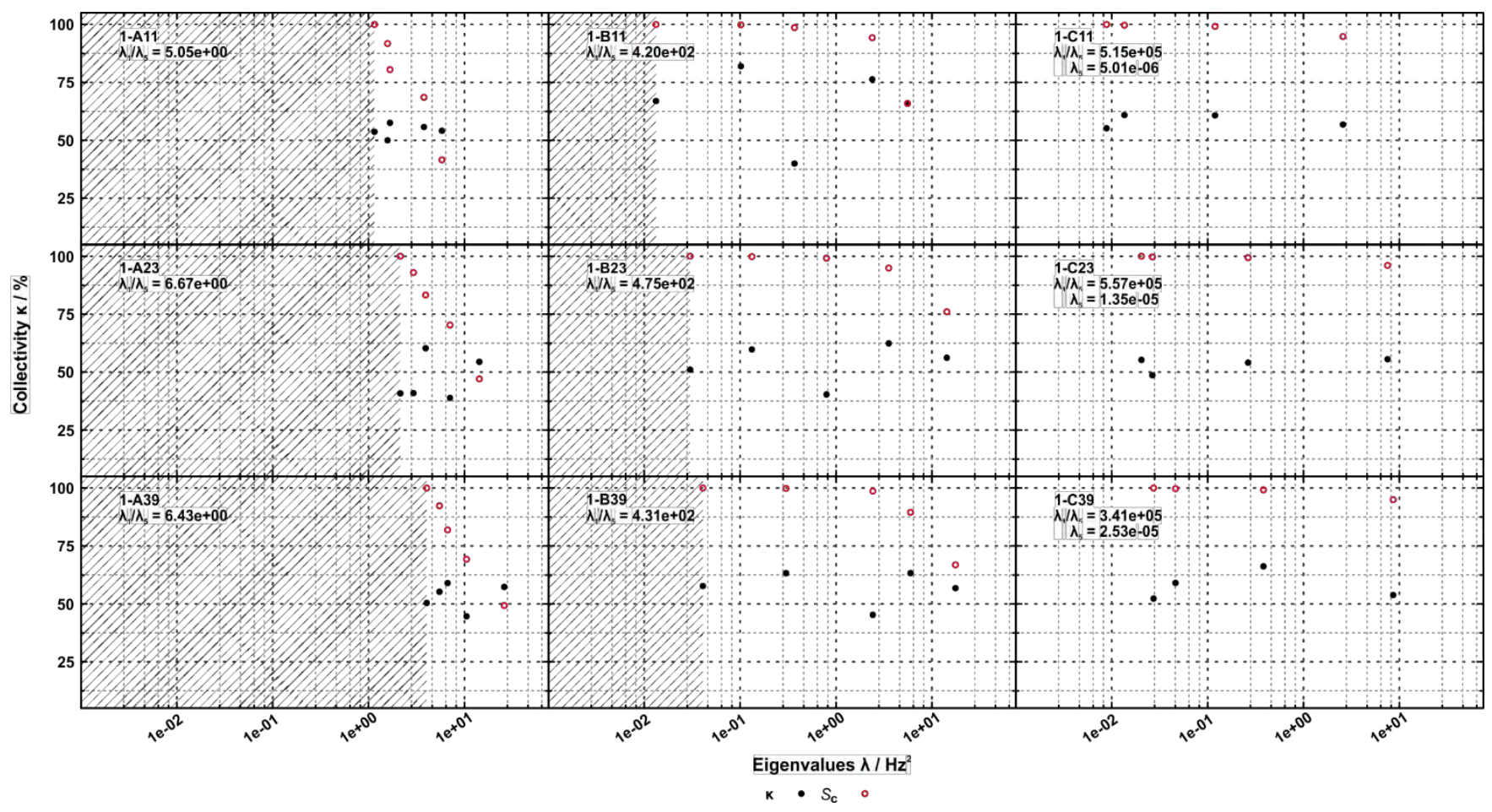

Figure 5. SECONDA plots for three runs of 1-A to 1-C using 11, 23 and 39 RDCs. The collectivities $\kappa(\bullet)$ are plotted with respect to the eigenvalues $\lambda$ of the RDC covariance matrix. All eigenvalues $\lambda_{n}<1 \mathrm{e}-3$ are not plotted to retain the same scaling for all SECONDA plots. In addition the cumulative sum $S_{c}(\circ)$ of the eigenvalues are plotted to estimate the contribution of the principle variances to the overall variance of the RDC matrix. In run $1-B 11$, the points of $\kappa(\lambda 1)$ and $S_{c}(\lambda 1)$ overlap coincidentally.

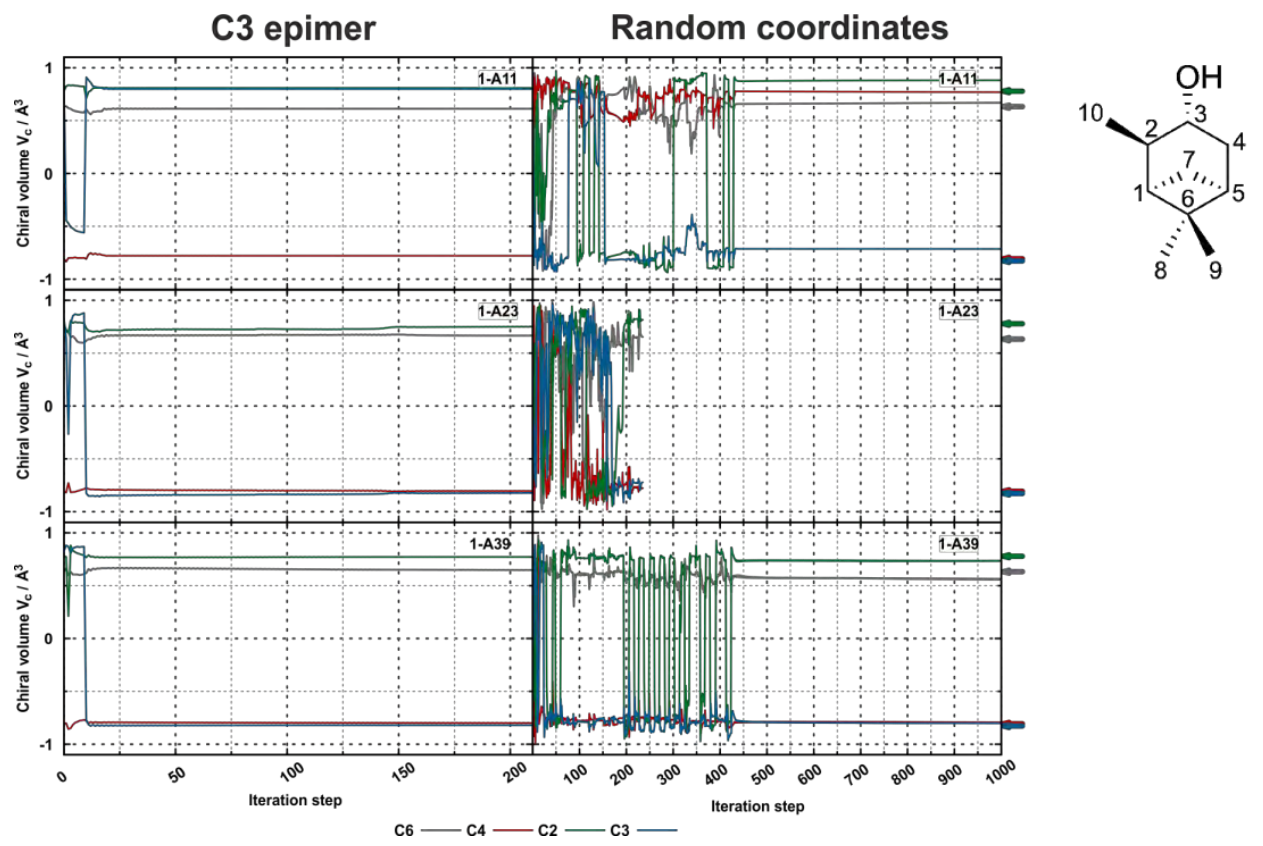

Figure 6. Trajectories of the chiral volumes of interest for setup 1-A. All plots on the left hand side monitor optimization starting with the deliberately inverted structure at $\mathrm{C} 3$. Additionally the two attached carbons (C2 and C4) and the quaternary carbon C6 (only two ${ }^{1} D_{\mathrm{CC}}$ in all runs) are shown. The correct chiral volumes are indicated by an arrow at the right hand side. Note that the results for starting from random coordinates are shown on the left hand side.

Centers with strongly distorted tetrahedral geometry will result in larger deviations and therefore are less likely to represent a proper result. For 1-A11 the rmsd of C3 is $0.20 \AA^{3}$ while well-defined centers like C4 in the same run have an rmsd of $0.05 \AA^{3}$.The runs utilizing ${ }^{\mathrm{n}} D_{\mathrm{HH}}$ RDCs (1-A17 to 39, see SI Figure S-12 for 1-A23 and 1-A31) all show their final inversion in iteration step 10. All 
following iterations confirm the correct stereochemistry with low values on the rmsds of chiral volumes.

The progression of the optimization trajectory can be monitored closely by the Monte-Carlo bootstrapping (see SI Figure S-10 for the corresponding plots for all runs). For all runs of 1-A, the orientation and structure parameters quickly achieve convergence even though there are some fluctuations in the initial iterations. In addition to the stop criteria the $S_{\text {overall }}$ parameter converges towards its theoretically expected limit of 1.0 (since the RDCs were predicted by using a single rigid structure in the absence of heterogeneities) with deviations due to counteractions of the RDC information and the holonomic terms in the redundant internal coordinates. This constantly leads to small fluctuations in the coordinates and subsequently a slight underestimation of the $S_{\text {overall }}$ parameter.

Notably, the above optimization results are only marginally affected by the starting geometry. When starting from a set of random coordinates (or a flat geometry without any stereochemical information), the calculations may take several hundred iteration steps to achieve similar thresholds of convergence. However, the runs arrive at the same geometries and orientation conditions as the corresponding runs starting from the $\mathrm{C} 3$ epimer. The 1-A11 run starting from a random geometry again results in the wrong configuration, while all runs involving ${ }^{\mathrm{n}} D_{\mathrm{HH}} \mathrm{RDC}$ s produce the correct configuration (see Figure 6). The resulting geometries and orientation parameters coincide very closely with the results from the previous runs (see SI section 2.3), highlighting the robustness of the algorithm.

From the setup 1-A with artificial data it can be clearly concluded that the correct stereochemical configuration is obtained from sufficiently large and well-sampled RDC data result in the correct stereochemical configuration, while poor data may either not converge at all or lead to a distorted geometry as in the $\mathrm{C} 3$ case.

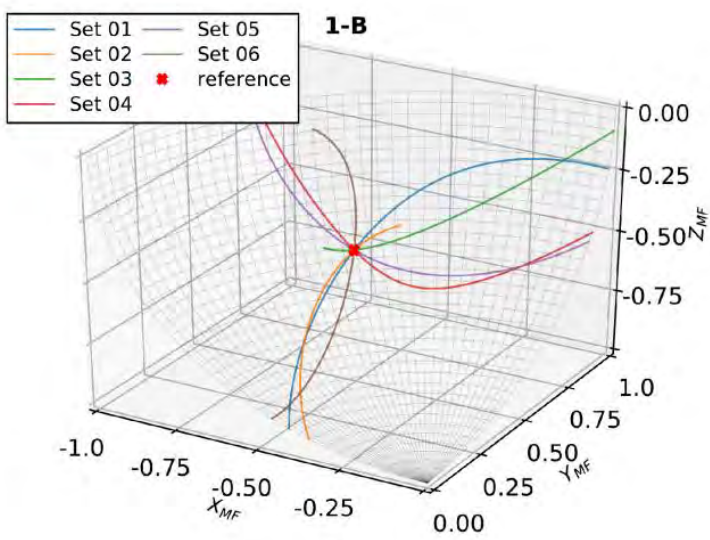

Application on IPC: realistic data. The applicability to a more realistic number and choice of RDC sets is investigated in setups 1B and 1-C. Both underlying data sets are based on synthetic data with tensor orientations from real samples. Setup 1-B represents $\mathrm{RDC}$ data with linearly independent orientations using six alignment conditions. Setup 1-C on the other side showcases high linear dependency by using similar alignment conditions. For 1-B, the SECONDA plots (see Figure 5) show large differences to the respective 1-A runs in the magnitude of the eigenvalues $\lambda_{1}$ to $\lambda_{5}$. The collectivities show no extraordinary values for the runs containing ${ }^{n} D_{\mathrm{HH}}$ RDCs. As expected for data without experimental error and structural noise no more than five non-zero eigenvalues are found. In 1-A the $\lambda_{1} / \lambda_{5}$ gap was lower than ten for all runs. This was interpreted as excellent linear independence. Setup 1-B on the other hand shows $\lambda_{1} / \lambda_{5}$ gaps larger than 400 , indicating that the experimental data used in the synthetic setups 1-B (and 1-C) exhibit a lower degree of linear independence than the corresponding artificial runs (1-A). This is mainly apparent in the magnitudes of the eigenvalues $\lambda_{2}-\lambda_{5}$, while $\lambda_{1}$ is of comparable size for the individual runs of 1-A and 1-B. The question for a suitable measure for linear independence arises, thus we have chosen a threshold of $1 \%$ contribution of the individual eigenvalues in the overall variance in order to be considered. In setup 1-A the fifth (smallest) eigenvalue contributes at least $6 \%$, which we consider significant. In setup 1-B the $1 \%$ threshold is already reached with eigenvalue $\lambda_{3}$ for most runs, thus $\lambda_{4}$ and $\lambda_{5}$ have little contribution to the overall variance. The apparent reduction to only three notable eigenvalues (roughly equating to only three significantly contributing independent alignment conditions) should still be sufficient for a TITANIA optimization as noted previously be Ruan et al. ${ }^{30}$ For setup 1-C the fifth eigenvalue is more than five orders of magnitude smaller than the first one leading to extraordinarily large $\lambda_{1} / \lambda_{5}$ gaps. The eigenvalue with a contribution of more than $1 \%$ is $\lambda_{2}$ in all cases.

Figure 7. Threedimensional plot of possible directions for the $\mathrm{C} 3 \mathrm{H} 3 \mathrm{RDC}$ vector based on synthetic data from experimental orientations (see SI equation 6a). The intersection of the cones represent the mean vector orientation obtained by RDC data. The exact same intersection is found for 1 $\mathrm{B}$ and 1-C as encountered in the artificial RDC data (1-A), which perfectly matches the orientation of the C3H3 RDC vector in the reference structure. The orientation data used to generate the plot were all obtained from the reference structure. 
This indicates that only two independent (based on our deliberate threshold) orientations are present; thus structure elucidation is expected to fail using TITANIA. Thus it needs to be investigated whether the optimizations in TITANIA confirm our expectations from the SECONDA analysis.

Surprisingly, the chiral volume trajectories of 1-B and 1-C for the C3-epimer as starting geometry (see SI Figure S-12) show nearly the exact same progression as 1-A did. The differences are rather small (except 1-B39 vs. 1-C39) and can only be revealed by examination of the exact chiral volumes (for example $\mathrm{C} 3$ iteration 1: $\left.V_{c}[1-B 11]=-0.44438 \AA^{3}, V_{c}[1-C 11]=-0.44500 \AA^{3}\right)$. The final structures of 1-B11 and 1-C11 show the same distorted tetrahedron with incorrect configuration at C3 (as shown in Figure 12 for tubocurarine).

The similarity of results is, furthermore, illustrated by the intersection cones generated by all possible $\theta, \phi$ combinations obtained when solving equation 2 for the respective RDCs of C3-H3. When plotting all possible orientations of this RDC vector calculated in the setups 1-B and 1-C on a unit sphere, two unique intersections are found, coinciding with the reference vector orientation in the molecular frame (see Figure 7). ${ }^{81-82}$ These are the two degenerate solutions of the RDC vector (correct orientation and its inverse).

Interestingly, one intercept is found for the individual runs despite the rather dramatic dependence in 1-C. This illustrates, that as long as homogeneous RDC data (i.e. free of heterogeneous behavior (in this case error)) are available, even small differences in the alignment conditions (like in 1-C) are sufficient for the optimization with TITANIA. The impact of heterogeneities in the form of data uncertainty on the intersection and thereby on the optimization using TITANIA will be discussed below on the runs 1-E and 1-F. The counter acting of the linear dependence and heterogeneities in the RDCs on the ability to superiorly define the RDC vectors is observed in the trajectory plot (see SI Figure S-11). Here changes in the mean vector length $(r m s d(R))$ as well as the fluctuations in the change of the spherical coordinates $(\operatorname{rms} d(\mathrm{p}))$ increase with linear dependence. No perfect intersection of the individual cones (defined by the respective alignment media) will be obtained since the Monte-Carlo bootstrap adds errors to the RDCs. This originates from the fact that 1-A will still define the orientation with high accuracy due to an expected error cancelation by the 20 RDC sets. When reducing the number of sets this error cancelation will not be as efficient as in 1-A. This reduction of the error cancelation is even stronger when the degree of linear dependence grows (1C). This can be observed when calculating the average of the Monte-Carlo mean vector lengths for all RDCs vectors in one setup $\left(\left\langle R_{1-\mathrm{A}}\right\rangle=0.965,\left\langle R_{1-\mathrm{B}}\right\rangle=0.906,\left\langle R_{1-\mathrm{C}}\right\rangle=0.862\right)$.

As before for setup 1-A, all runs were also performed with random starting coordinates (see SI figure S-14 and S-15). The optimization trajectories are much rougher for 1-B and for 1-C, and generally show even more inversion steps before reaching convergence. Interestingly, some 1-B runs arrive at the enantiomeric form, while converging towards the correct relative configuration. This is not unexpected due to the nature of the random start and because RDCs are inherently unable to determine the absolute configuration without enantiospecific interactions with the alignment medi- um being used to drive the optimization towards the correct absolute configuration. ${ }^{72}$ For 1-B11 again the incorrect (relative) configuration at C3 is observed. The deliberately chosen poor linear independence of runs 1-C has a stronger impact for the optimization, than observed for 1-B. 1-C11 shows two incorrect configurations and 1-C23 shows one. The other 1-C runs converge to the correct configurations and show that even relying on such a limited set of contributing alignment media still allows for a successful structure optimization with TITANIA. The results, however, must carefully be evaluated and the optimization trajectory may depend strongly on the RDC data set and choice of starting geometry.

The analyses detailed above demonstrate the ability of TITANIA to optimize the structure of IPC resulting in the correct configuration when using a sufficient amount of error-free data.

Inducing experimental error. The next step is now to investigate whether and how experimental error together with varying degrees of linear dependence impacts the optimizations. Therefore Gaussian random noise is added as synthetic error to the RDCs in setups 1-A to 1-C generating the setups 1-D to 1-F, respectively. It is expected that 1-F (1-C with error) will not converge to the correct solution since data were deliberately chosen to be linearly dependent. It is reported that the propagation of errors strongly depends on the linear (in-)dependence of the RDC data sets. ${ }^{24}$ Since the contribution of the linear independence in alignment conditions is known and SECONDA is capable of detecting heterogeneities in 1-E to 1-F, the impact of errors can directly be analyzed.

The SECONDA plots of the RDC data in the presence of error are shown in the SI (section 2.2.1). While the eigenvalues $\lambda_{1}$ to $\lambda_{5}$ of 1-D only show minor changes compared to 1-A the collectivities change significantly. As expected the $\lambda_{5} / \lambda_{6}$-gaps now become observable due to the RDC errors in 1-D. It shrinks in magnitude with increasing set sizes. As the $\kappa_{\mathrm{q}}$ values associated with the latter eigenvalues drop in magnitude with increasing set sizes, we assume heterogeneities of a localized nature to be present.

The SECONDA data for 1-E unexpectedly show barely any change in magnitude or the respective $\kappa_{\mathrm{q}}$ value of the first three eigenvalues when compared to 1-B. The eigenvalues $\lambda_{4}$ and $\lambda_{5}$ on the other side increase while $\kappa_{q}$ drops when adding more RDCs and the sixth eigenvalue remains smaller than the cutoff of 1e-9. The latter is also true for the runs 1-F. Most likely, the low degree of linear independence in the RDC data leads to heterogeneities being observed in the first eigenvalues and not generating additional eigenvalues. This behavior was not observed by Hus et al., who reported that heterogeneities will result in additional eigenvalues but $\lambda_{1}$ to $\lambda_{5}$ remain nearly unchanged for linear independent data (i.e. see $1-D) .^{50}$

The data containing errors on the RDCs show huge differences to the error-free data. The trajectories of setup 1-F did not show convergence or ended in highly distorted geometries. Thus linearly dependent data with experimental error will not lead to a valid solution as expected. The respective trajectories are not plotted but are shown in the supporting information. Instead the vector addition algorithm, only optimizing the orientation of the RDC vectors, is shown for setup 1-E (explanation see SI section 1.3.5). 


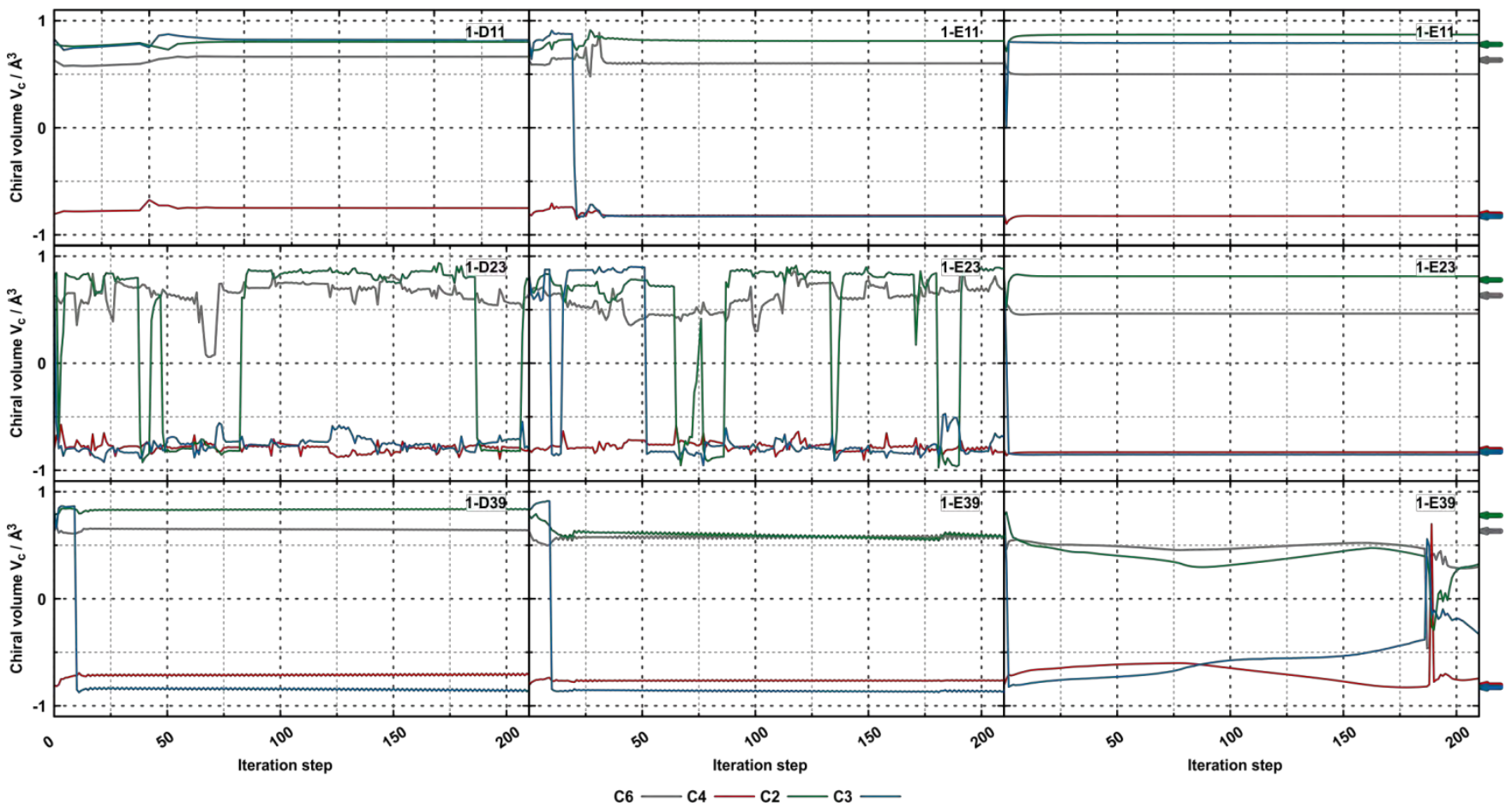

Figure 8. Trajectories of the chiral volumes of interest for the runs 1-D and 1-E using 11, 23 and 39 RDCs. All plots monitor optimization starting with the deliberately inverted structure at $\mathrm{C} 3$, the two attached carbons (C2 and C4) and the quaternary carbon $\mathrm{C} 6$ (only two ${ }^{1} D_{\mathrm{CC}}$ in all runs). In addition to the standard algorithm to generate Cartesian coordinates (redundant internal coordinates, left and middle panel) the vector addition algorithm was also used (right panel). The vector addition shows a much smoother trajectory. The correct chiral volumes are indicated by an arrow at the right hand side.

The trajectories are now more distinct for individual runs with the same set sizes. In the runs utilizing 11 RDCs the previous behavior is observed again; with the curious exception that 1-E11 in the redundant coordinate algorithm (middle panel) now achieves the inversion to the correct stereochemistry, while the center is obtained in the wrong configuration in the vector addition algorithm. The runs with $23 \mathrm{RDCs}$ undergo several flips of methyl group $\mathrm{RDC}$ vectors $(\mathrm{C} 2 / \mathrm{C} 6)$ for the 1-D and 1-E sub run in the redundant coordinates algorithm. While the interchange of the methyl groups at $\mathrm{C} 6$ is of no consequence for structure determination here, the inversion at $\mathrm{C} 2$ leads to a wrong configuration (see structures in supplementary material). Thus it is essential to check the final structure for inconsistencies (distorted geometries or wrong bond length), but also an inspection of the chiral volume trajectories is recommended to detect such problems. This - admittedly problematic - behavior of the optimization when using redundant coordinates is resolved when using the vector addition algorithm (see Figure 8 right hand side). The correct relative configuration is obtained quickly. The downside of this algorithm is that only a subset of coordinates (those for which RDC data is available) is optimized. This can lead to distortions in the overall structure (see inset of 1-E39), while retaining the proper orientations between the RDC vectors. The problems in properly defining methyl group orientations are not encountered when extending the set sizes (see 1-D39 and 1-E39). This shows that a structure optimization resulting in the correct final configuration is possible not only with ideal and error-free data but also with more realistic data of moderate linear independence.

As mentioned before the 1-F runs did not converge or lead to proper structures. These differences between the runs (1-D/1-E vs. 1-F) are also observed when looking at the intersection of the possible orientation cones (see Figure 9). In the case of 1-E five of the six alignment media intersect very close to the reference orientation. In contrast, in setup 1-F no common unique intersection is found anymore due to the added errors (compare Figure 7 to Figure 9). An extended discussion of the stop criteria and the respective trajectories are presented in the supporting information (see section 2.2.3). The data in this chapter leads to the conclusion that the quality of data as chosen in 1-E (experimental orientations with moderate dependency with (artificial) experimental error) are already sufficient to obtain the correct structure, whereas linearly dependent data (1-F with artificial experimental error) do not allow to converge to the correct structure. Thus already a limited set of different orientations may be adequate for structure optimization of small organic compounds if enough RDCs are available and the sampled alignment conditions are sufficiently independent. Larger set sizes increase the accuracy of the refined structure when experimental heterogeneities are present. In the absence of experimental errors the need for linear independence is reduced to an extent, while the presence of such sources of heterogeneity exacerbates this requirement. 

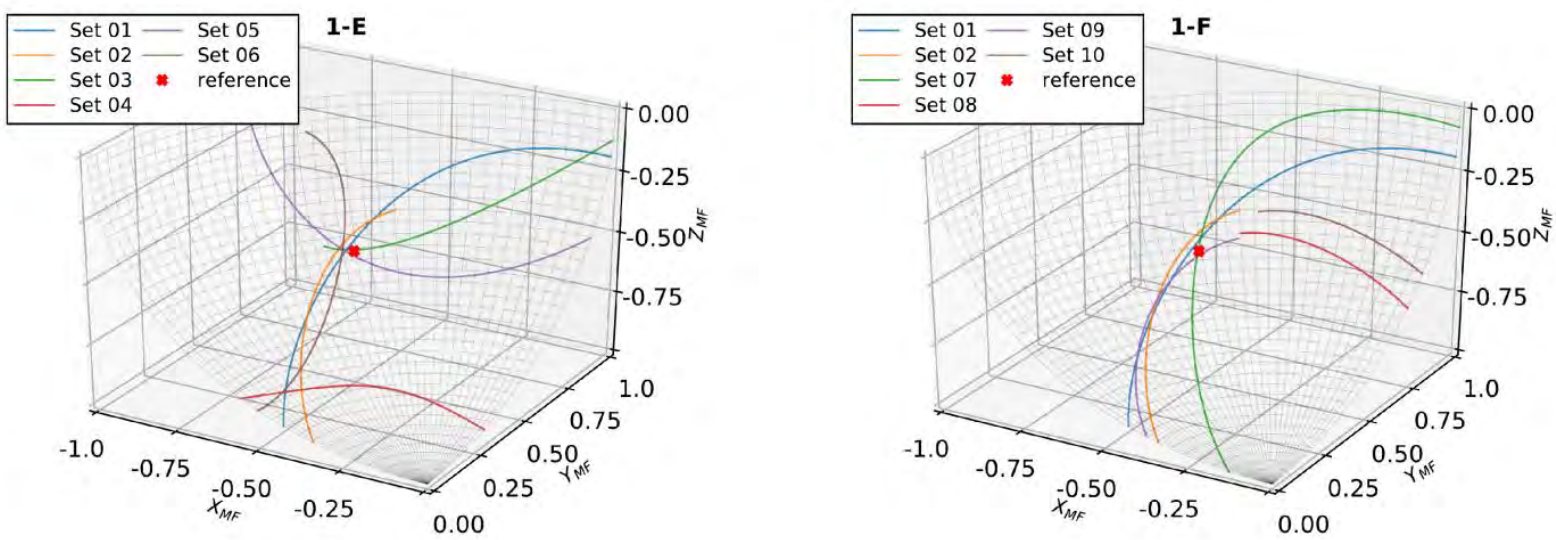

Figure 9. Three-dimensionall plot of possible directions for the $\mathrm{C} 3 \mathrm{H} 3 \mathrm{RDC}$ vector based on the synthetic data utilizing $11 \mathrm{RDCs}$ each with varying degrees of linear dependency (see SI equation 6a). The intersection of the cones represent the mean vector orientation obtained by RDC data. Using RDC data containing heterogeneities the optimized solution stronly depends on the difference of the alignment conditions. Therefore the intersection found for 1-E (the linearly less dependent data set) is similar to the correct one (see Figure 7), with only one alignment set (set 04) showing a large deviation. 1-F (the data set that is linearly dependent on purpose) on the other hand has no well defined array fitting the data. Again the orientation of the $\mathrm{C} 3 \mathrm{H} 3 \mathrm{RDC}$ vector in the reference structure is plotted as red cross. The orientation data used to generate the plot were all obtained from the reference structure.

Application on Tubocurarine. Tubocurarine 2 is a cyclic neurotoxin that consists of four cyclic subunits containing aromatic rings and piperidinium ions. The crystal structure was solved 1975 by Reynold and Palmer ${ }^{83}$ and in 2019 tubocurarine was used for a proof of concept by Immel et al. for a configurational and conformational analysis using fc-rDG/DDD with special interest on the orientation of the rings $\mathbf{C}$ and $\mathbf{F}$ with respect to $\mathbf{A} / \mathbf{B}$ and $\mathbf{D} / \mathbf{E}$. It was shown that at least three linearly independent RDC sets have to be used (successively) to properly assign the correct relative configuration. $^{53}$

Tubocurarine is capable of undergoing large conformational changes, emphasizing the need to jointly determine the conformation and relative configuration. The compound features two separated, rigid RDC subunits (rings $\mathbf{A} / \mathbf{B}$ and $\mathbf{E} / \mathbf{D}$ ), which are connected by flexible linkers (bridging methylene groups 18 and 36 and rings $\mathbf{C}$ and F). Lastly, the introduction of heteronuclear couplings to nitrogen is accompanied by large relative errors.

Eight alignment conditions were used to generate artificial RDCs which were directly used (2-A) and extended to model experimental errors as described above (2-B). Each set contained a total of 30 RDCs using ${ }^{1} D_{\mathrm{CH}},{ }^{1} D_{\mathrm{NH}},{ }^{1} D_{\mathrm{CN}}$ and all ${ }^{\mathrm{n}} D_{\mathrm{HH}}$ couplings within a cutoff radius of $3.0 \AA$. Notably, the methylene groups 18 and 36 were only defined by ${ }^{1} D_{\mathrm{CH}} \mathrm{RDC}$ (see SI for the exact assignment). The RDCs were calculated from a single rigid structure and the respective errors were explicitly generated to not mimic any conformational noise, i.e. no additional down-scaling of RDCs in flexible groups was added. The effect of the large relative error is immediately observed in the difference of the SECONDA plots for 2-A and 2-B. The 2-A plot shows a $\lambda_{1} / \lambda_{5}$ ratio which lies in-between the ratios of 1-A (around 5) and 1-B (above 400). All eigenvalues contribute with more than $1 \%$, with the fourth eigenvalue having a contribution of $5.9 \%$ to the overall variance, and all collectivities $\kappa$ are larger than $50 \%$. This suggests a high degree of linear independence for 2-A. Interestingly, the pattern of the eigenvalue-collectivity pairs changes drastically when random noise is added in $\mathbf{2 - B}$. As expected more than five non-zero eigenvalues are observed, revealing heterogeneities in the RDC sets used for the run 2-B. The eigenvalue $\lambda_{1}$ drops in collectivity while $\lambda_{2}$ remains nearly unchanged. $\lambda_{3}$ increases in size but the collectivity is reduced by a factor of $\sim 3$. In addition, the fourth eigenvalue $\lambda_{4,2-\mathrm{B}}$ has no correspondent in run 2-A, while $\lambda_{4,2-A}$ is represented in $\lambda_{5,2-B}$. Similarly, $\lambda_{5,2-\mathrm{A}}$ ends up close to the values of $\lambda_{7,2-\mathrm{B}}$. This means, that $\lambda_{4,2-\mathrm{B}}$ and $\lambda_{6,2-\mathrm{B}}$ reflect the random noise and $\kappa_{3,2-\mathrm{B}}$ is strongly reduced due to the error. To support this interpretation, the respective squared eigenmodes are plotted in the supporting information. Both the SECONDA and eigenmodes plots reflect the strong influence of the large ${ }^{1} D_{\mathrm{CN}}$ relative errors.

The chiral volumes also show the expected behavior. No fast inversions of all stereogenic centers can be observed anymore and multiple inversions occur on N1. For 2-A the chiral volume of the nitrogen centers remain stable after the final configuration was reached. In 2-B small fluctuations are observed throughout the whole optimization.

All chiral centers end up in the correct configuration. Only the pro-chiral center $\mathrm{C} 18$ does not converge to the correct assignment of the diastereotopic protons. This behavior was already observed for C3 of IPC. After a stable configuration is achieved on C18 the chiral volume is extraordinarily high for an unstrained $\mathrm{sp}^{3}$ carbon. Figure 12 shows the respective centers for $\mathbf{2 - A}$ and $\mathbf{2 - B}$ (solid structures) and the reference structure used to derive the RDCs (transparent). The planar geometry, resulting in a chiral volume close to $1.0 \AA^{3}$, shows that the correct vector orientations are found 


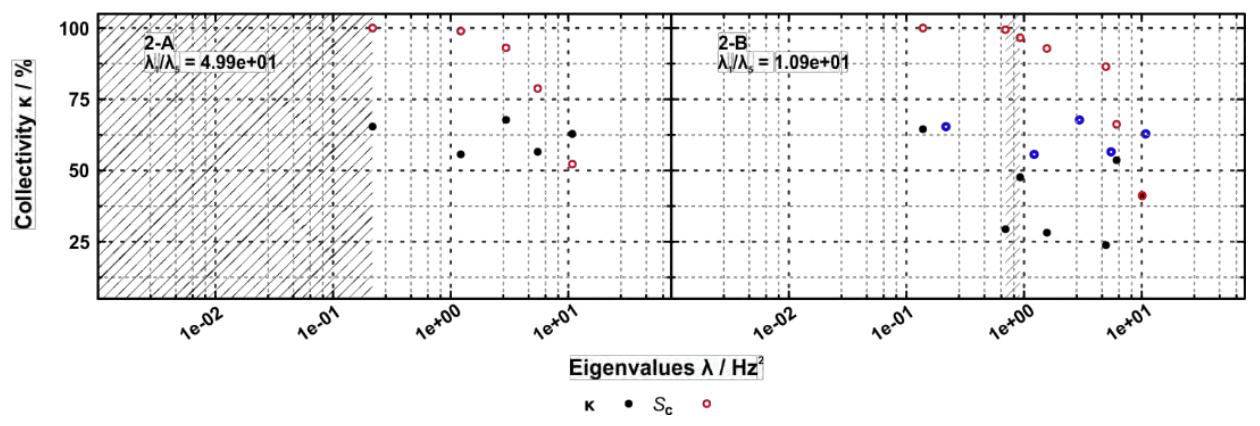

Figure 10. SECONDA plots for the two runs 2-A (without noise) and 2-B (with Gaussian random noise) of tubocurarine 2. Plotted are the collectivities $\kappa(\bullet)$ with respect to the eigenvalues $\lambda$ of the RDC covariance matrix (blue eigenvalues in the right hand side plot are from 2-A). In addition the cumulative sum $(\circ)$ of the eigenvalues were plotted to measure the participation of the principle variances to the overall variance of the RDC matrix.

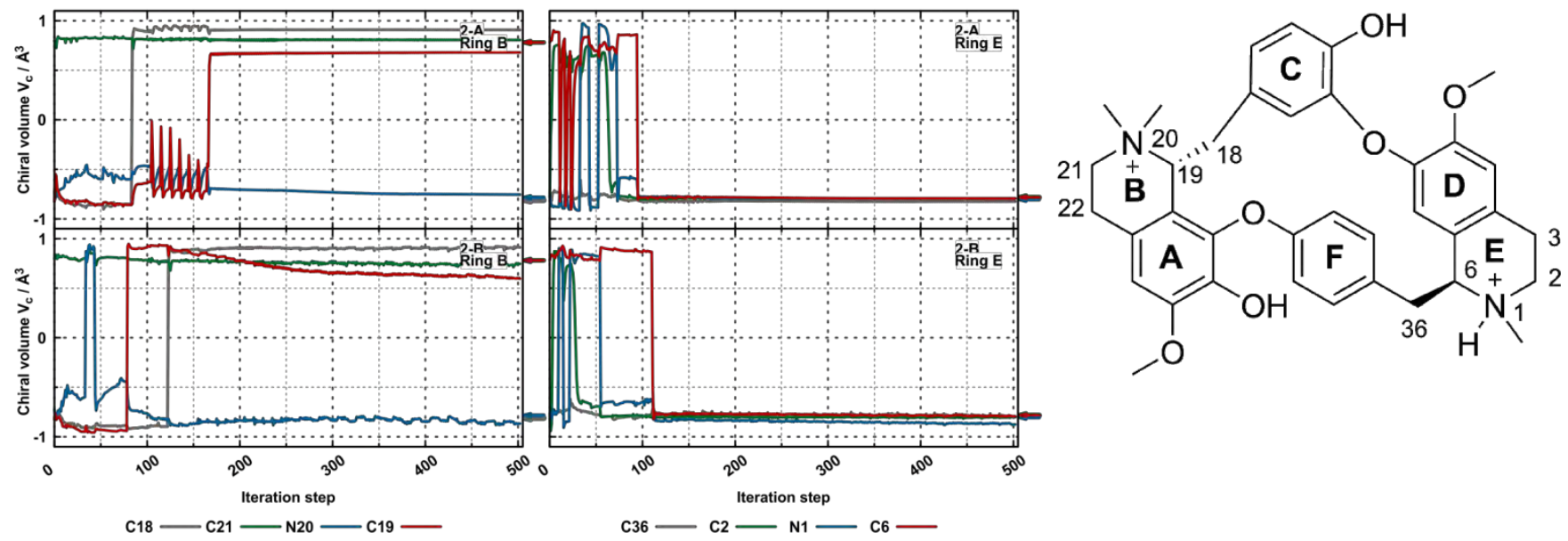

Figure 11. Trajectories of the chiral volumes of interest (rings $\mathbf{B}$ and $\mathbf{E}$ ) for the runs 2-A (top side) and 2-B (bottom side). Additionally the respective methylene groups C18 and C36 are plotted.

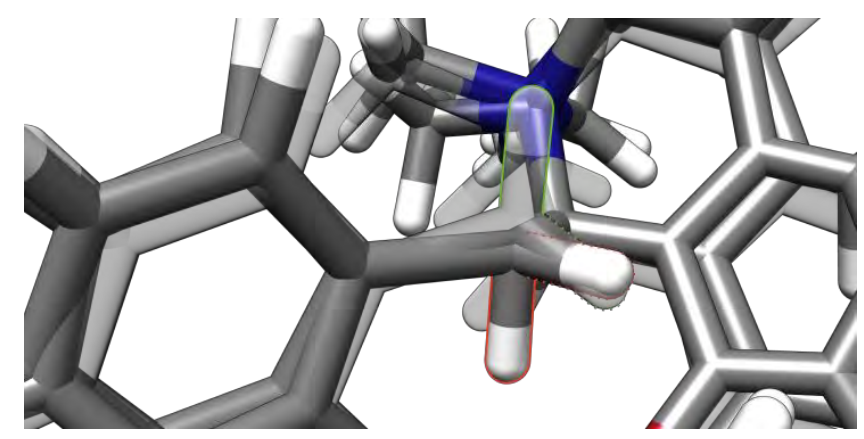

consequence of the high relative errors. Additional plots showing the reorientation of the aromatic rings $\mathbf{C}$ and $\mathbf{D}$ as well as the corresponding full trajectories and Monte-Carlo rmsds are given in the supporting information.

Application on Strychnine. The final example is strychnine 3 which contains six chiral and six pro-chiral centers. In contrast to IPC 1, in which inversions of stereogenic centers will not lead to large distortions of the overall geometry, this example showcases a compound with chiral centers bridging two or more rings. Inversions on centers like C16 in strychnine 3 will lead to major distortions of the whole scaffold's geometry. The effect of the resulting large rearrangements on the overall optimization in combination with the full use of the stop criteria, set to a feasible limit to demonstrate the capability to detect convergence, will be discussed here. Eleven artificial RDC sets with 43 RDCs were used for the TITANIA run (called 3-A here for consistency with the other examples). The 43 RDCs were again generated by using ${ }^{1} D_{\mathrm{CH}}$ couplings and long range ${ }^{n} D_{\mathrm{HH}}$ couplings limited to those with internuclear distances of not more than $3 \AA$. As in the previous examples the SECONDA plot was used to confirm the linear independence in combination to the absence of errors in the RDC sets (see SI Figure S-22). 

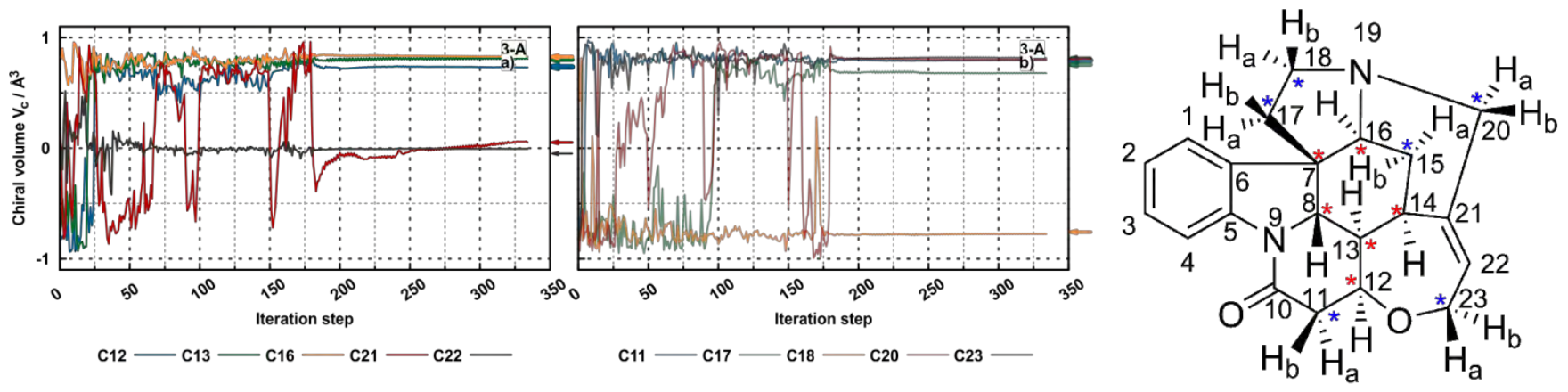

Figure 13. Trajectories of the chiral volumes of interest for strychnine (run 3-A). The subplot a) shows the chiral volumes of the tertiary and quaternary carbons ( $\mathrm{C} 21$ and $\mathrm{C} 22$ are $\mathrm{sp}^{2}$ carbons and therefore should have a chiral volume $V_{\mathrm{c}}=0 \AA^{3}$ ). The second subplot b) shows the chiral volumes of the pro-chiral centers.

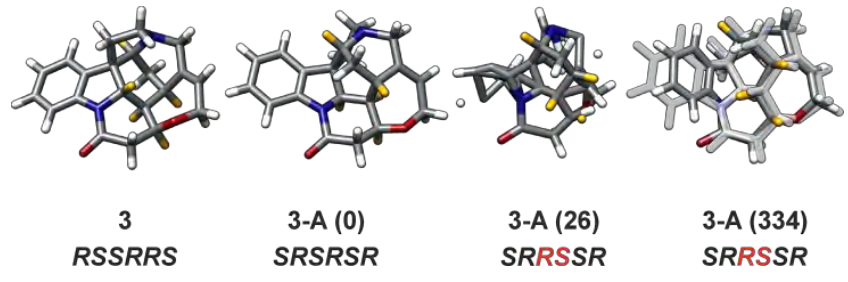

Figure 14. Comparison of different strychnine structures, with hydrogens of the chiral centers marked in gold. The first structure is the reference structure taken from literature. The other structures are encountered at different points of the iterations (denoted in brackets) in run 3-A with the second structure depicting the deliberately "inverted" scaffold of the starting geometry. As the resulting final structure is the enantiomer, it was superimposed with the enantiomer of 3 (transparent) generated from the literature coordinates. For a better assessment of the structures the stereo descriptors of the chiral centers, which are ordered according to the atom numbering, are reported. The red descriptors are the inverted ones.

The eigenvalues of 3-A show excellent linear independence, comparable to the IPC runs 1-A. All eigenvalues contribute to the overall variance and do not show extraordinarily low collectivities. This implies that a fast convergence should be possible.

As starting geometry for this test (see Figure 14, 3-A(0)), four stereogenic centers of the scaffold were deliberately inverted (for more information see experimental section). The optimization of 3-A shows much more complex trajectories than the previous runs. The majority of the chiral centers invert in the first part of the optimization (see Figure 13 subplot a). This can also be seen in the 3D structure of 3-A at iteration 26 in Figure 14, where the chiral centers have been rearranged into the correct configuration by TITANIA. In contrast, the $\mathrm{sp}^{2}$ carbon $\mathrm{C} 21$ shows large fluctuations and loses the expected planar geometry. This is likely elicited by the late convergence into the correct diastereotopic assignment and tetrahedral geometry of the neighboring pro-chiral center C20. Figure 14 illustrates an example for the large distortions of the 3D structure accompanied. The structure first loses its molecular shape. This is impressively observed at the aryl ring of iteration step 26 in Figure 14. In the following steps TITANIA reassembles strychnine achieving the correct vector orientations and relative configuration. As observed before for runs of 1-B using random start coordinates, the enantiomer of $\mathbf{3}$ is obtained in this run. The relatively late inversion of the pro-chiral $\mathrm{C} 20$, however, would be of little consequence for structure elucidation if it was overlooked.

Strychnine shows how TITANIA is able to perform optimizations on rather complex compounds. In the course of the optimization the molecular shape is lost and rebuild to a proper structure based on the RDCs.

\section{CONCLUSION}

We demonstrate the use of multiple alignment media to determine the vector orientations solely from RDC data and use them to optimize the structure of small organic compounds with the newly developed software TITANIA on three representative examples. It was expected that such an approach is only feasible if enough linearly independent data sets are available. We could show here, that in an error-free scenario the linear independence is not as crucial in the structure refinement of IPC (setup 1-C). Interestingly, above a certain threshold an increase in the number of alignment media does not affect the result in the absence of error. The set size, however, does: especially the inclusion of long-range couplings has a significant beneficial effect on the optimization (see 1-X11 runs with $\mathbf{X}$ equals $\mathbf{A}$ to $\mathbf{C}$ ). More importantly, the process is not only possible using reasonable starting geometries, but also is successful when using random coordinates. This shows the enormous potential of the multiple alignment approach proposed here as this allows structure determination even in the absence of any prior information on stereogenic relations.

These very encouraging findings change when more realistic data with (artificial) experimental error are used. Here linearly dependent data may not lead to a reliable result (see 1-F). Random inversions may occur in the optimization due to the incomplete sampling of possible alignments. RDC data with better linear independence (see 1-D and 1-E) converge to the correct solution when using long-range couplings. Even few long-range couplings, which are indeed available from modern experiments, ${ }^{48}$ will improve accuracy in the vector orientations. The few examples which converged to the wrong configuration showed strong distortions of the tetrahedral geometries indicating the correct structure by showing the inverse RDC vector solution at the respective stereogenic center thus allowing an easy identification by the operator. 
The assessment of the linear independence within the TITANIA protocol is based on the SECONDA approach. Here the linear (in)dependence is analyzed simultaneously to possible heterogeneities. While initially developed to asses internal motions leading to heterogeneities captured in the RDCs, we use the combination of the eigenvalues and eigenmodes to identify linear (in)dependence of the alignment media used. Here the eigenvalues can be assessed by the distribution of the eigenvector elements. A global character (uniform distribution) shows eigenvalues that are caused by a change in orientation, thus showing a new linear independent orientation. Localized characters (few elements with large magnitude) are more likely to be related to heterogeneities in terms of structural or RDC noise.

Additionally to the small, rigid geometry like IPC (1) larger compounds with separated stereogenic domains and flexibility like tubocurarine (2) or complex and dependent scaffold geometries like strychnine (3) can be optimized using TITANIA. The trajectories become more complex and show more inversions until the final configuration is reached. Future work aims at using real experimental datasets. Modern alignment media, especially stimuli responsive media, in combination with the development of new pulse sequences, allowing the measurement of a large variety of longrange homo- and heteronuclear RDCs, build a toolbox to test TITANIA in the limits of modern NMR spectroscopy and structure elucidation. Additionally TITANIA reveals not only relative configurations and conformations of small organic compounds, but also allows for the determination of local order parameters, which will certainly be a very valuable source of information on dynamics in the future. All of the above features make TITANIA an optimal tool for the interpretation of RDC data in the context of a full structure elucidation.

\section{EXPERIMENTAL SECTION}

For the structure optimizations using TITANIA different kinds of RDC data are used. One is referred to as synthetic RDC set, where a set is a collection of RDCs under the same alignment condition. These synthetic values are predicted by utilizing a reference structure and alignment parameters derived from RDC sets reported in literature. The second kind of RDCs is referred to as artificial. The respective $\mathrm{RDC}$ sets are predicted by randomly generated orientation parameters $\left(\mathrm{Azz}<10-2, \mathrm{R}=[0,2 / 3], \alpha=\left[0^{\circ}, 180^{\circ}\right], \beta=\right.$ $\left[-180^{\circ}, 180^{\circ}\right], \gamma=\left[-180^{\circ}, 180^{\circ}\right]$ with ZYZ Euler convention for the AF to $\mathrm{MF}$ rotation). Unless stated otherwise, the optimizations were performed without proper stop criteria (only maximum number of iterations) for a better demonstration of the trajectories. The implemented stop criteria will be discussed in the results section.

The first example to demonstrate the concepts of TITANIA is isopinocampheol 1 which is well studied with RDCs. The input structure for this task was generated by inverting the chiral center C3 of a structure published earlier. ${ }^{75} 20$ artificial RDC sets were predicted using the literature structure and used directly in the setup called 1-A (information on how RDCs were chosen, see below). Additional data sets were generated from literature data, ${ }^{37,}$ ${ }^{75,84}$ resulting in one setup with the goal to achieve linear independence (1-B) and another one expected to show linear dependence
(1-C) by utilizing only six RDC sets each. The assignments of the sets to the respective references can be found in the supporting information (see Table S-1). Additionally, the RDC setups 1-A to 1-C were also optimized starting from random coordinates with $\mathrm{x}$, $y, z=[0.0,1.0] \AA$ to show the ability of TITANIA to optimize structures without any prior stereochemical information.

To simulate experimental error of the RDCs Gaussian distributed random numbers $(\sigma=0.5 \mathrm{~Hz})$ was added to the setups 1-A to 1C resulting in 1-D (1-A with errors), 1-E (1-B with errors) and 1-F (1-C with errors). The error for the homonuclear RDCs were downscaled due to their lower $D_{\max }$ compared to ${ }^{1} D_{\mathrm{CH}}$ couplings (common scaling factor for ${ }^{1} D_{\mathrm{CC}} 25 \%$ and ${ }^{n} D_{\mathrm{HH}} 50 \%$ ).

All setups were optimized in five individual runs utilizing different set sizes (run 1-A11, A17, A23, A31 and A39 and analogous for setups $\mathbf{B}$ to $\mathbf{F}$ ) where the respective identifier encodes the number of RDCs per set of alignment conditions. The basis for all sets consists of all ${ }^{1} D_{\mathrm{CH}}$ (and derived methyl ${ }^{1} D_{\mathrm{CC}}$ ) couplings (1-A11). Additional six ${ }^{3} D_{\mathrm{HH}}$ couplings wrapping the structure once (1A17), all ${ }^{2} D_{\mathrm{HH}}$ and ${ }^{3} D_{\mathrm{HH}}$ couplings (1-A23), an experimental set defined by 31 RDCs reported in literature ${ }^{48}$ (1-A31) and all possible ${ }^{n} D_{\mathrm{HH}}$ couplings (1-A39) were chosen as meaningful set sizes. The RDC nuclei of the individual runs are listed in the supporting information (see Table S-81).

Tubocurarine 2 was optimized using eight artificial RDC sets with 37 RDCs each (see Table S-87). The RDCs were predicted using the published, modified solid state structure ${ }^{53}$ with the original labels. ${ }^{83}$ The chiral centers C6 and C19 were inverted and the resulting structure was minimized using the MMFF94 force field. The RDC sets contain the ${ }^{1} D_{\mathrm{CH}}$ and additional nine ${ }^{\mathrm{n}} \mathrm{D}_{\mathrm{HH}}$ couplings of the piperidinium protons. As for IPC, artificial error was added to the underlying RDC sets of run 2-A to generate 2-B. The added errors (Gaussian distributed with $\sigma=0.5 \mathrm{~Hz}$ ) were not scaled for ${ }^{1} D_{\mathrm{NC}}$ and ${ }^{1} D_{\mathrm{NH}}$ to discuss the impact of the larger and more localized relative errors (especially ${ }^{1} D_{\mathrm{CN}}$ ) and we only rejected random numbers exceeding $1.5 \mathrm{~Hz}$ to prevent unrealistic high errors.

Strychnine 3 was optimized using eleven artificial RDC sets with 43 RDCs each in run 3-A (see Table S-91). The RDCs were predicted using a structure from literature. ${ }^{7}$ The RDC sets were generated using all ${ }^{1} D_{\mathrm{CH}}$ couplings and the ${ }^{2 / 3} D_{\mathrm{HH}}$ couplings of the protons with distances lower than $3 \AA$. The input structure was generated by inverting the chiral centers $\mathrm{C} 7, \mathrm{C} 8, \mathrm{C} 14$ and $\mathrm{C} 16$ of the ring scaffold. The resulting structure was minimized using the MMFF94 force field.

All input and output files containing the presented data (RDCs, structures, alignment conditions and SECONDA) are available as supplementary material. More details on the TITANIA protocol, the keywords to choose, further SECONDA analyses and all (further) trajectories can be found in the supporting information. As supplementary material we provide a zip archive, that contains all input and output files used here.

The source code for the TITANIA program as well as precompiled binaries are available from the authors upon request. 


\section{ASSOCIATED CONTENT}

Supporting Information. This material is available free of charge via the Internet at http://pubs.acs.org.

Detailed description of the TITANIA protocol (chapter 1), experimental orientations in 1-B/1-C (table S-1), SECONDA data of IPC 1 (section 2.2.1), all orientations associated to IPC using the C3-epimer as starting structure (section 2.2.2-2.2.5), data and discussion when starting from random coordinates (section 2.3), data and discussion when using non-default vector addition algorithm (section 2.4), detailed list of RDCs used for IPC (table S-81), final IPC structures (section 2.6), SECONDA eigenmodes for tubocurarine 2 (figure $\mathrm{S}$ 20 ), all orientations associated to tubocurarine (section 3.2), list of RDCs used for tubocurarine (table S-87), SECONDA plot for strychnine 3 (figure S-22), all orientations associated to strychnine (section 4.2), list of RDCs used for strychnine (table S-91), content of supplementary material zip-archive (chapter 5).

\section{AUTHOR INFORMATION}

\section{Corresponding Author}

Prof. Dr. Christina. M. Thiele

Clemens-Schöpf-Institut für Organische Chemie und Biochemie

Technical University of Darmstadt, Alarich-Weiss-Str. 16, 64287

Darmstadt (Germany); http://orcid.org/0000-0001-7876-536X; Email: cthiele@thielelab.de

\section{Authors}

\section{Felix A. Roth}

Clemens-Schöpf-Institut für Organische Chemie und Biochemie Technical University of Darmstadt, Alarich-Weiss-Str. 16, 64287 Darmstadt (Germany)

\section{Dr. Volker Schmidts}

Clemens-Schöpf-Institut für Organische Chemie und Biochemie Technical University of Darmstadt, Alarich-Weiss-Str. 16, 64287 Darmstadt (Germany); https://orcid.org/0000-0002-7195-312X

\section{Author Contributions}

The manuscript was written through contributions of all authors.

\section{ACKNOWLEDGMENT}

The authors thank Rafael Brüschweiler for helpful discussions on SECONDA.

\section{ABBREVIATIONS}

RDC residual dipolar couplings, MFA model-free approach, TITANIA TITANIA performs iterative analysis of independent alignments, SECONDA self-consistency of dipolar couplings analysis, AF alignment frame, VF vector frame, MF molecular frame.

\section{REFERENCES}

1. Levitt, M. H., Spin Dynamics: basics of nuclear magnetic resonance. 2 ed.; Wiley VCH: 2008.

2. Neuhaus, D.; Williamson, M. P., The Nuclear Overhauser Effect in Structural and Conformational Analysis. 2000.

3. Karplus, M., Contact Electron - Spin Coupling of Nuclear Magnetic Moments. J. Chem. Phys. 1959, 30, 11-15.

4. Bifulco, G.; Dambruoso, P.; Gomez-Paloma, L.; Riccio, R., Determination of relative configuration in organic compounds by NMR spectroscopy and computational methods. Chem. Rev. 2007, 107 (9), 37443779 .

5. Tolman, J. R.; Flanagan, J. M.; Kennedy, M. A.; Prestegard, J. H., NMR evidence for slow collective motions in cyanometmyoglobin. Nature Structural Biology 1997, 4 (4), 292-297.

6. Tjandra, N.; Bax, A., Direct measurement of distances and angles in biomolecules by NMR in a dilute liquid crystalline medium. Science 1997, 278 (5340), 1111-4.

7. Thiele, C. M.; Berger, S., Probing the Diastereotopicity of Methylene Protons in Strychnine Using Residual Dipolar Couplings. Org. Lett. 2003, 5 (5), 705-708.

8. Verdier, L.; Sakhaii, P.; Zweckstetter, M.; Griesinger, C., Measurement of long range $\mathrm{H}, \mathrm{C}$ couplings in natural products in orienting media: a tool for structure elucidation of natural products. J. Magn. Reson. 2003, $163(2), 353-9$.

9. Aroulanda, C.; Boucard, V.; Guibé, F.; Courtieu, J.; Merlet, D., Weakly oriented liquid-crystal NMR solvents as a general tool to determine relative configurations. Chem. Eur. J. 2003, 9 (18), 4536-9.

10. Böttcher, B.; Thiele, C. M., Determining the Stereochemistry of Molecules from Residual Dipolar Couplings (RDCs). Encyclopedia of Magnetic Resonance 2012.

11. Chen, K.; Tjandra, N., The Use of Residual Dipolar Coupling in Studying Proteins by NMR. In NMR of Proteins and Small Biomolecules. Topics in Current Chemistry, G., Z., Ed. SpringerBerlin: Berlin, Heidelberg, 2001; Vol. 326.

12. Ban, D.; Sabo, T. M.; Griesinger, C.; Lee, D., Measuring dynamic and kinetic information in the previously inaccessible supra-tau(c) window of nanoseconds to microseconds by solution NMR spectroscopy. Molecules 2013, 18 (10), 11904-37.

13. Huben, K.; Jewgiński, M.; Pabis, A.; Paluch, P.; Luy, B.; Jankowski, S., The structure of cyclolinopeptide $\mathrm{A}$ in chloroform refined by $\mathrm{RDC}$ measurements. J. Pept. Sci. 2014, 20 (11), 901-907.

14. Salmon, L.; Blackledge, M., Investigating protein conformational energy landscapes and atomic resolution dynamics from NMR dipolar couplings: a review. Rep Prog Phys 2015, 78 (12), 126601.

15. Fredersdorf, M.; Göstl, R.; Kolmer, A.; Schmidts, V.; Monecke, P.; Hecht, S.; Thiele, C. M., Exploring the conformational space of bridgesubstituted dithienylcyclopentenes. Chem. Eur. J. 2015, 21 (41), 14545-54.

16. Saupe, A., Recent Results in the Field of Liquid Crystals. Angew. Chem. Int. Ed. 1968, 7 (2), 97-112.

17. Kramer, F.; Deshmukh, M. V.; Kessler, H.; Glaser, S. J., Residual dipolar coupling constants: An elementary derivation of key equations. Conc. Magn. Reson. A 2004, 21A (1), 10-21.

18. Losonczi, J. A.; Andrec, M.; Fischer, M. W. F.; Prestegard, J. H., Order Matrix Analysis of Residual Dipolar Couplings Using Singular Value Decomposition. J. Magn. Reson. 1999, 138 (2), 334-342.

19. Thiele, C. M.; Schmidts, V.; Böttcher, B.; Louzao, I.; Berger, R.; Maliniak, A.; Stevensson, B., On the Treatment of Conformational Flexibility when Using Residual Dipolar Couplings for Structure Determination. Angew. Chem. Int. Ed. 2009, 48 (36), 6708-6712.

20. Sun, H.; Reinscheid, U. M.; Whitson, E. L.; d'Auvergne, E. J.; Ireland, C. M.; Navarro-Vázquez, A.; Griesinger, C., Challenge of Large-Scale Motion for Residual Dipolar Coupling Based Analysis of Configuration: The Case of Fibrosterol Sulfate A. J. Am. Chem. Soc. 2011, 133 (37), 14629-14636.

21. Tolman, J. R., A Novel Approach to the Retrieval of Structural and Dynamic Information from Residual Dipolar Couplings Using Several Oriented Media in Biomolecular NMR Spectroscopy. J. Am. Chem. Soc. 2002, 124 (40), 12020-12030.

22. Briggman, K. B.; Tolman, J. R., De Novo Determination of Bond Orientations and Order Parameters from Residual Dipolar Couplings with High Accuracy. J. Am. Chem. Soc. 2003, 125 (34), 10164-10165.

23. Meiler, J.; Prompers, J. J.; Peti, W.; Griesinger, C.; Brüschweiler, R. Model-Free Approach to the Dynamic Interpretation of Residual Dipolar Couplings in Globular Proteins. J. Am. Chem. Soc. 2001, 123 (25), 6098-6107.

24. Peti, W.; Meiler, J.; Brüschweiler, R.; Griesinger, C., Model-Free Analysis of Protein Backbone Motion from Residual Dipolar Couplings. J. Am. Chem. Soc. 2002, 124 (20), 5822-5833. 
25. Lipari, G.; Szabo, A., Model-free approach to the interpretation of nuclear magnetic resonance relaxation in macromolecules. 1 . Theory and range of validity. J. Am. Chem. Soc. 1982, 104 (17), 4546-4559.

26. Lipari, G.; Szabo, A., Model-free approach to the interpretation of nuclear magnetic resonance relaxation in macromolecules. 2. Analysis of experimental results. J. Am. Chem. Soc. 1982, 104 (17), 4559-4570.

27. Lakomek, N.-A.; Walter, K. F. A.; Farès, C.; Lange, O. F.; de Groot, B. L.; Grubmüller, H.; Brüschweiler, R.; Munk, A.; Becker, S.; Meiler, J.; Griesinger, C., Self-consistent residual dipolar coupling based model-free analysis for the robust determination of nanosecond to microsecond protein dynamics. J. Biomol. NMR 2008, 41 (3), 139.

28. Sabo, T. M.; Smith, C. A.; Ban, D.; Mazur, A.; Lee, D.; Griesinger, C., ORIUM: Optimized RDC-based Iterative and Unified Model-free analysis. J. Biomol. NMR 2014, 58 (4), 287-301.

29. Yao, L.; Vögeli, B.; Torchia, D. A.; Bax, A., Simultaneous NMR Study of Protein Structure and Dynamics Using Conservative Mutagenesis. J. Phys. Chem. B 2008, 112 (19), 6045-6056.

30. Ruan, K.; Briggman, K. B.; Tolman, J. R., De novo determination of internuclear vector orientations from residual dipolar couplings measured in three independent alignment media. J. Biomol. NMR 2008, 41 (2), 61-76.

31. Marx, A.; Böttcher, B.; Thiele, C. M., Enhancing the orienting properties of poly(gamma-benzyl-L-glutamate) by means of additives. Chem. Eur. J. 2010, 16 (5), 1656-63.

32. Meyer, N.-C.; Krupp, A.; Schmidts, V.; Thiele, C. M.; Reggelin, M., Polyacetylenes as Enantiodifferentiating Alignment Media. Angew. Chem. Int. Ed. 2012, 51 (33), 8334-8338.

33. Merle, C.; Kummerlöwe, G.; Freudenberger, J. C.; Halbach, F.; Stöwer, W.; Gostomski, C. L. v.; Höpfner, J.; Beskers, T.; Wilhelm, M.; Luy, B., Crosslinked Poly(ethylene oxide) as a Versatile Alignment Medium for the Measurement of Residual Anisotropic NMR Parameters. Angew. Chem. Int. Ed. 2013, 52 (39), 10309-10312.

34. Lei, X.; Xu, Z.; Sun, H.; Wang, S.; Griesinger, C.; Peng, L.; Gao, C.; Tan, R. X., Graphene Oxide Liquid Crystals as a Versatile and Tunable Alignment Medium for the Measurement of Residual Dipolar Couplings in Organic Solvents. J. Am. Chem. Soc. 2014, 136 (32), 11280-11283.

35. Jeziorowski, S.; Thiele, C. M., Poly- $\gamma$-p-Biphenylmethyl-Glutamate as Enantiodifferentiating Alignment Medium for NMR Spectroscopy with Temperature-Tunable Properties. Chem. Eur. J. 2018, 24 (58), 15631-15637.

36. Schwab, M.; Schmidts, V.; Thiele, C. M., Thermoresponsive Alignment Media in NMR Spectroscopy: Helix Reversal of a Copolyaspartate at Ambient Temperatures. Chem. Eur. J. 2018, 24 (54), 14373-14377.

37. Schwab, M.; Herold, D.; Thiele, C. M., Polyaspartates as Thermoresponsive Enantiodifferentiating Helically Chiral Alignment Media for Anisotropic NMR Spectroscopy. Chem. Eur. J. 2017, 23 (58), 14576-14584.

38. Gil-Silva, L. F.; Santamaría-Fernández, R.; Navarro-Vázquez, A.; Gil, R. R., Collection of NMR Scalar and Residual Dipolar Couplings Using a Single Experiment. Chem. Eur. J. 2016, 22 (2), 472-476.

39. $\quad$ Reller, M.; Wesp, S.; Koos, M. R. M.; Reggelin, M.; Luy, B., Biphasic Liquid Crystal and the Simultaneous Measurement of Isotropic and Anisotropic Parameters by Spatially Resolved NMR Spectroscopy. Chem. Eur. J. 2017, 23 (54), 13351-13359.

40. Kurz, M.; Schmieder, P.; Kessler, H., HETLOC, an Efficient Method for Determining Heteronuclear Long-Range Couplings with Heteronuclei in Natural Abundance. Angew. Chem. Int. Ed. 1991, 30 (10), 13291331.

41. Uhrin, D.; Batta, G.; Hruby, V. J.; Barlow, P. N.; Kövér, K. E., Sensitivity- and Gradient-Enhanced Hetero ( $\omega 1)$ Half-Filtered TOCSY Experiment for Measuring Long-Range Heteronuclear Coupling Constants. J. Magn. Reson. 1998, 130 (2), 155-161.

42. Meissner, A.; Sørensen, O. W., Measurement of J(H,H) and longrange $\mathrm{J}(\mathrm{X}, \mathrm{H})$ coupling constants in small molecules. Broadband XLOC and $\mathrm{J}$ HMBC. Magn. Reson. Chem. 2001, 39 (1), 49-52.

43. Fehér, K.; Berger, S.; Kövér, K. E., Accurate determination of small one-bond heteronuclear residual dipolar couplings by F1 coupled HSQC modified with a G-BIRD(r) module. J. Magn. Reson. 2003, 163 (2), 340-346.
44. Kövér, K. E.; Fehér, K., Measurement of one-bond heteronuclear dipolar coupling contributions for amine and diastereotopic methylene protons. J. Magn. Reson. 2004, 168 (2), 307-313.

45. Enthart, A.; Freudenberger, J. C.; Furrer, J.; Kessler, H.; Luy, B., The CLIP/CLAP-HSQC: pure absorptive spectra for the measurement of onebond couplings. J. Magn. Reson. 2008, 192 (2), 314-22.

46. Thiele, C. M.; Bermel, W., Speeding up the measurement of onebond scalar (1J) and residual dipolar couplings (1D) by using non-uniform sampling (NUS). J. Magn. Reson. 2012, 216, 134-143.

47. Castañar, L.; Sauri, J.; Williamson, R. T.; Virgili, A.; Parella, T., Pure in-phase heteronuclear correlation NMR experiments. Angew. Chem. Int. Ed. 2014, 53 (32), 8379-82.

48. $\quad$ Sinnaeve, D.; Ilgen, J.; Di Pietro, M. E.; Primozic, J. J.; Schmidts, V.; Thiele, C. M.; Luy, B., Probing Long-Range Anisotropic Interactions: a General and Sign-Sensitive Strategy to Measure (1) H-(1) H Residual Dipolar Couplings as a Key Advance for Organic Structure Determination. Angew. Chem. Int. Ed. 2020, 59 (13), 5316-5320.

49. Koźmiński, W.; Nanz, D., Sensitivity Improvement and New Acquisition Scheme of Heteronuclear Active-Coupling-Pattern-Tilting Spectroscopy. J. Magn. Reson. 2000, 142 (2), 294-299.

50. Hus, J.-C.; Brüschweiler, R., Principal component method for assessing structural heterogeneity across multiple alignment media. J. Biomol. NMR 2002, 24 (2), 123-132.

51. Hus, J.-C.; Peti, W.; Griesinger, C.; Brüschweiler, R., SelfConsistency Analysis of Dipolar Couplings in Multiple Alignments of Ubiquitin. J.Am. Chem. Soc. 2003, 125 (19), 5596-5597.

52. Cornilescu, G.; Ramos Alvarenga, R. F.; Wyche, T. P.; Bugni, T. S.; Gil, R. R.; Cornilescu, C. C.; Westler, W. M.; Markley, J. L.; Schwieters, C. D., Progressive Stereo Locking (PSL): A Residual Dipolar Coupling Based Force Field Method for Determining the Relative Configuration of Natural Products and Other Small Molecules. ACS Chem. Biol. 2017, 12 (8), 2157-2163.

53. Immel, S.; Köck, M.; Reggelin, M., Configurational Analysis by Residual Dipolar Coupling Driven Floating Chirality Distance Geometry Calculations. Chem. Eur. J. 2018, 24 (52), 13918-13930.

54. Tzvetkova, P.; Sternberg, U.; Gloge, T.; Navarro-Vázquez, A.; Luy, B., Configuration determination by residual dipolar couplings: accessing the full conformational space by molecular dynamics with tensorial constraints. Chem. Sci. 2019, 10 (38), 8774-8791.

55. Fares, C.; Lingnau, J. B.; Wirtz, C.; Sternberg, U., Conformational Investigations in Flexible Molecules Using Orientational NMR Constraints in Combination with (3)J-Couplings and NOE Distances. Molecules 2019, 24 (23).

56. Di Pietro, M. E.; Sternberg, U.; Luy, B., Molecular Dynamics with Orientational Tensorial Constraints: A New Approach to Probe the Torsional Angle Distributions of Small Rotationally Flexible Molecules. J. Phys. Chem. B 2019, 123 (40), 8480-8491.

57. Saupe, A.; Englert, G., High-Resolution Nuclear Magnetic Resonance Spectra of Orientated Molecules. Phys. Rev. Lett. 1963, 11 (10), 462464.

58. Saupe, A., Kernresonanzen in kristallinen Flüssigkeiten und in kristallinflüssigen Lösungen. Teil I. In Zeitschrift für Naturforschung A, 1964; Vol. 19, p 161.

59. Burnell, E. E., Effects of Interaction between Molecular Internal Motion and Reorientation on Nmr of Anisotropic Liquids. J. Magn. Reson. 1980, 39 (3), 461-480.

60. Emsley, J. W.; Luckhurst, G. R., The effect of internal motion on the orientational order parameters for liquid crystalline systems. Mol. Phys. 1980, 41 (1), 19-29.

61. Burnell, E. E.; de Lange, C. A.; Mouritsen, O. G., Effects of intramolecular motion on the magnetic resonance of anisotropic liquids: The equivalence of kinetic and equilibrium statistical mechanical approaches. J. Mag. Reson. 1982, 50 (2), 188-196.

62. Thiele, C. M.; Marx, A.; Berger, R.; Fischer, J.; Biel, M.; Giannis, A., Determination of the Relative Configuration of a Five-Membered Lactone from Residual Dipolar Couplings. Angew. Chem. Int. Ed. 2006, 45 (27), 4455-4460.

63. Skrynnikov, N. R; Goto, N. K.; Yang, D.; Choy, W.-Y.; Tolman, J. R.; Mueller, G. A.; Kay, L. E., Orienting domains in proteins using dipolar 
couplings measured by liquid-state NMR: differences in solution and crystal forms of maltodextrin binding protein loaded with $\beta$-cyclodextrin11Edited by $P$. E. Wright. J. Mol. Biol. 2000, 295 (5), 1265-1273.

64. Merlet, D.; Emsley, J. W.; Lesot, P.; Courtieu, J., The relationship between molecular symmetry and second-rank orientational order parameters for molecules in chiral liquid crystalline solvents. J. Chem. Phys. 1999, 111 (15), 6890-6896.

65. Zweckstetter, M.; Bax, A., Prediction of Sterically Induced Alignment in a Dilute Liquid Crystalline Phase: Aid to Protein Structure Determination by NMR. J. Am. Chem. Soc. 2000, 122 (15), 3791-3792.

66. Ibáñez de Opakua, A.; Klama, F.; Ndukwe, I. E.; Martin, G. E.; Williamson, R. T.; Zweckstetter, M., Determination of Complex SmallMolecule Structures Using Molecular Alignment Simulation. Angew. Chem. Int. Ed. 2020, 59 (15), 6172-6176.

67. Peng, C.; Ayala, P. Y.; Schlegel, H. B.; Frisch, M. J., Using redundant internal coordinates to optimize equilibrium geometries and transition states. $J$. Comput. Chem. 1996, 17 (1), 49-56.

68. Bakken, V.; Helgaker, T., The efficient optimization of molecular geometries using redundant internal coordinates. J. Chem. Phys. 2002, 117 (20), 9160-9174.

69. Eyring, H., The resultant electric moment of complex molecules. Phys. Rev. 1932, 39 (4), 746--748.

70. Exclusion distances are implemented as a linear term equivalent to bond lengths, when interatomic distances fall below a predefined threshold (on average $~ 70 \%$ of standard 1-3 distances).

71. Havel, T. F.; Kuntz, I. D.; Crippen, G. M., The theory and practice of distance geometry. Bull. Math. Biol. 1983, 45 (5), 665-720.

72. Berger, R.; Courtieu, J.; Gil, R. R.; Griesinger, C.; Köck, M.; Lesot, P.; Luy, B.; Merlet, D.; Navarro-Vázquez, A.; Reggelin, M.; Reinscheid, U. M.; Thiele, C. M.; Zweckstetter, M., Is Enantiomer Assignment Possible by NMR Spectroscopy Using Residual Dipolar Couplings from Chiral Nonracemic Alignment Media?-A Critical Assessment. Angew. Chem. Int. Ed. 2012, 51 (33), 8388-8391.

73. Lakomek, N. A.; Carlomagno, T.; Becker, S.; Griesinger, C.; Meiler, J., A Thorough Dynamic Interpretation of Residual Dipolar Couplings in Ubiquitin. J. Biomol. NMR 2006, 34 (2), 101-115.

74. Sass, J.; Cordier, F.; Hoffmann, A.; Rogowski, M.; Cousin, A.; Omichinski, J. G.; Löwen, H.; Grzesiek, S., Purple Membrane Induced Alignment of Biological Macromolecules in the Magnetic Field. J. Am. Chem. Soc. 1999, 121 (10), 2047-2055.

75. Marx, A.; Schmidts, V.; Thiele, C. M., How different are diastereomorphous orientations of enantiomers in the liquid crystalline phases of PBLG and PBDG: a case study. Magn. Reson. Chem. 2009, 47 (9), 734-740.

76. F.A. Roth, V. Schmidts, J. Rettig, C.M. Thiele, Manuscript in preparation.

77. Pearson, K., LIII. On lines and planes of closest fit to systems of points in space. The London, Edinburgh, and Dublin Philosophical Magazine and Journal of Science 1901, 2 (11), 559-572.

78. Hotelling, H., Analysis of a complex of statistical variables into principal components. Journal of Educational Psychology 1933, 24 (6), 417-441.

79. Brüschweiler, R, Collective protein dynamics and nuclear spin relaxation. J. Chem. Phys. 1995, 102 (8), 3396-3403.

80. The magnitude of the chiral Volume $|V c|=\left|a *\left(\begin{array}{lll}b & x & c\end{array}\right)\right|$ is calculated for all possible permutations of the bondvectors. The rmsd of all $|\mathrm{Vc}|$ is calculated to assess the geometry of the respective center. If the geometry is well defined the rmsd will result in a low value.

81. Ulmer, T. S.; Ramirez, B. E.; Delaglio, F.; Bax, A., Evaluation of Backbone Proton Positions and Dynamics in a Small Protein by Liquid Crystal NMR Spectroscopy. J. Am. Chem. Soc. 2003, 125 (30), 9179-9191.

82. Tolman, J. R.; Ruan, K., NMR Residual Dipolar Couplings as Probes of Biomolecular Dynamics. Chem. Rev. 2006, 106 (5), 1720-1736.

83. Reynolds, C. D.; Palmer, R. A., The crystal structure, absolute configuration and stereochemistry of (+)-tubocurarine dibromide methanol solvate: a potent neuromuscular blocking agent. Acta Cryst. B 1976, 32 (5), 1431-1439.
84. Hansmann, S.; Larem née Montag, T.; Thiele, C. M., Enantiodifferentiating Properties of the Alignment Media PELG and PBLG - A Comparison. Eur.J. Org. Chem. 2016, 2016 (7), 1324-1329. 


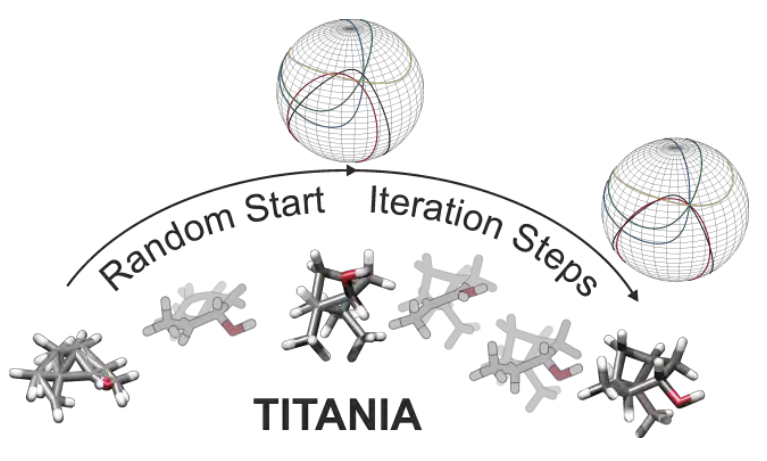




\title{
Supporting information TITANIA
}

\author{
Felix A. Roth, Volker Schmidts, Christina M. Thiele
}

\section{Contents}

$1 \quad$ TITANIA protocol $\quad 3$

1.1 Flowchart .................... . . 3

1.2 Input and output files . . . . . . . . . . . . . . . 4

1.2 .1 Input file . . . . . . . . . . . . . . . 4

1.2 .2 Output files . . . . . . . . . . . . . 7

1.3 Mathematics ................... . . 8

1.3.1 SECONDA weighting scheme . . . . . . . 8

1.3 .2 RDC theory . . . . . . . . . . . . . . 8

1.3.3 Information content of $Y \ldots \ldots \ldots 12$

1.3.4 Use of Redundant internal coordinates in TITANIA . . 13

1.3.5 Structures from vector addition . . . . . . . . . . 16

1.3.6 Statistics of directional data . . . . . . . . . . 17

2 Isopinocampheol (1) $\quad 19$

2.1 Orientations . . . . . . . . . . . . . . . . . . . . . 19

2.2 epi-C3 start structure . . . . . . . . . . . . . . . 22

2.2.1 SECONDA plots .................. 23

2.2 .2 Orientations . . . . . . . . . . . . . . . . . . 27

2.2.3 Optimization Trajectories . . . . . . . . . 48

2.2.4 Change in Orientation . . . . . . . . . . . . 54

2.2.5 Change induced by error . . . . . . . . . . . 59

2.3 Random coordinates structure . . . . . . . . . . . 63

2.3.1 Keyword adjustments . . . . . . . . . . 63

2.3.2 Output orientations . . . . . . . . . . . 64

2.3.3 Change in Orientation . . . . . . . . . . . . 73

2.3.4 Optimization Trajectories . . . . . . . . 76

2.4 Vector addition algorithm . . . . . . . . . . . . . 78

2.4.1 Output orientations . . . . . . . . . . . 78

2.4.2 Change in Orientation . . . . . . . . . . . 88

2.4.3 Optimization Trajectories ............ 91

2.5 RDC list . . . . . . . . . . . . . . . . . . . . . . . 94

2.6 Final structures . . . . . . . . . . . . . . . . . . . 95 
3 Tubocurarine (2) $\quad 97$

3.1 SECONDA eigenmodes . . . . . . . . . . . . . . . . . . 98

3.2 Orientations . . . . . . . . . . . . . . . . 100

3.2 .1 Tubocurarine run $2-\mathrm{A} \ldots \ldots \ldots . . \ldots 100$

3.2 .2 Tubocurarine run $2-\mathrm{B} \ldots \ldots . \ldots 101$

3.2.3 Change in Orientations . . . . . . . . . . . . 102

3.3 RDC list . . . . . . . . . . . . . . . . . . . . . 104

4 Strychnine (3) 105

4.1 SECONDA plot . . . . . . . . . . . . . . 105

4.2 Orientations . . . . . . . . . . . . . . . . . . 105

4.2.1 Strychnine run 3-A . . . . . . . . . . . . . 105

4.2.2 Change in Orientations . . . . . . . . . . 107

4.3 RDC list . . . . . . . . . . . . . . . . . 107

5 Content of Supplementary Material 109 


\section{TITANIA protocol}

\section{$1.1 \quad$ Flowchart}

TITANIA performs three major steps (parametrization, iterative refinement and Monte-Carlo bootstrap. See figure 4 in main text) which can be further extended as shown in fig. S-1.

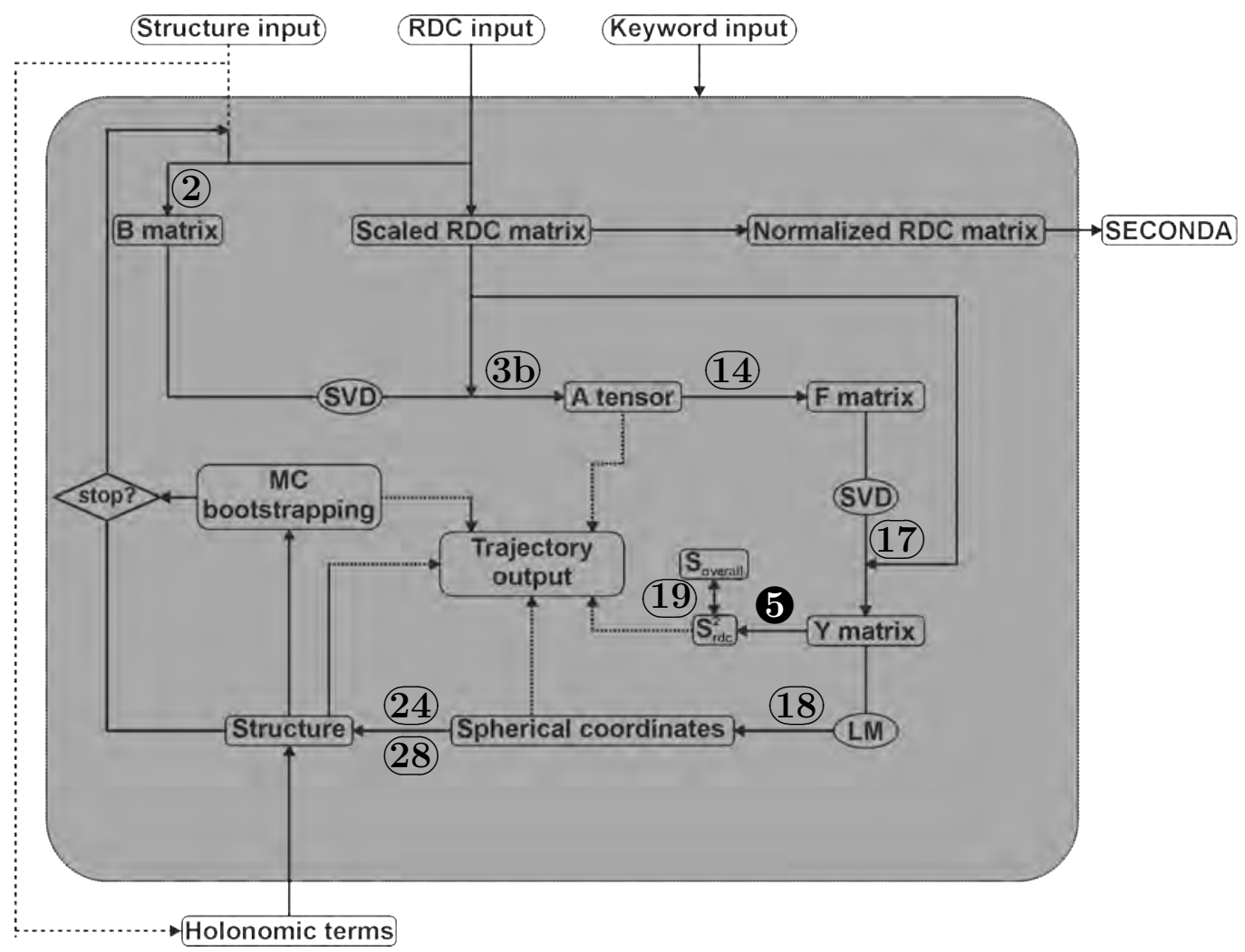

Figure S-1: Extended flowchart for TITANIA. The numbers shown in the scheme refer to the respective equation in the SI (gray) and main text (black).

The user has to define an initial structure (default: Cartesian coordinates and connectivity, see section 1.2.1) and the RDC data. In addition keywords can be used to adapt nearly every step of the TITANIA run to the current optimization. The first step is the parametrization of the structure, for which the holonomic terms (standard bond lengths, bond angles) are extracted from the initial input structure or the MMFF94 ${ }^{[1]}$ force field, the B-matrix is defined ${ }^{[2]}$ and initial parameters are calculated (e.g. bond lengths and 
$\left.D_{\max }\right)$. These initial parameters are used with the user defined RDCs to build a scaled and normalized RDC matrix, which is needed for the SECONDA analysis, the calculation of orientations and refined spherical harmonics $\mathbf{Y}$.

The alignment tensor $\mathbf{A}$ is calculated using the scaled RDC matrix $\mathbf{D}$ (for more information on the RDC matrix see section 1.3.2) and the normalized cosine matrix $\mathbf{B}$ and is analyzed via eigenvalue decomposition (describing the alignment frame AF). Using the resulting eigenvectors and rhombicity $R$ the F-matrix is constructed and utilized to determine the refined spherical harmonics $\mathbf{Y}$ by SVD of $\mathbf{F}$. To extract the spherical coordinates $\theta$ and $\phi$ from $\mathbf{Y}$ the Levenberg-Marquardt (LM) algorithm is used to find the Wigner rotation between the vector frame (VF) and molecular frame (MF). The structure can directly be derived from these angles using different algorithms, which can be chosen by the user by keywords. The default algorithm are the redundant internal coordinates (see section 1.3.4). An additional algorithm uses vector addition (see section 1.3.5) which leaves the carbon scaffold untouched. Thus no large changes required to correct wrong ring junctions (as in the strychnine example, see $\mathbf{3 - A}$ in the main text) are possible.

To check for convergence in the optimization and to estimate uncertainties of the previously calculated orientation and structure parameters a MonteCarlo bootstrap is performed. Some uncertainties can be determined directly (e.g. the uncertainty of the alignment tensor elements $\Delta A_{\mathrm{ij}}$ ). Others have to be estimated using Gaussian propagation of errors (e.g. the uncertainty of the spherical coordinates $\Delta p$ ). If convergence is reached, the iterative cylce is stopped (after $\mathrm{n}$ additional user defined cycles), an additional SECONDA analysis is performed (since the normalization factors depend on the structure) and the output files are finalized. In the course of the optimization the calculated parameters of all individual iteration steps are written to a trajectory file.

\subsection{Input and output files}

All input and output files of the individual runs reported in the main text are part of the supplementary material. The information content is described in the following.

\subsubsection{Input file}

TITANIA uses three types of inputs:

- Structure input: contains Cartesian coordinates of an initial structure. The structure contains labels for the individual atoms and follows a simple syntax: 


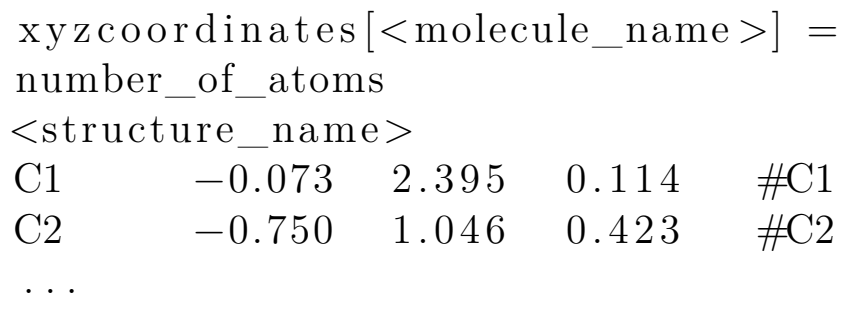

Additionally a connectivity matrix is defined.

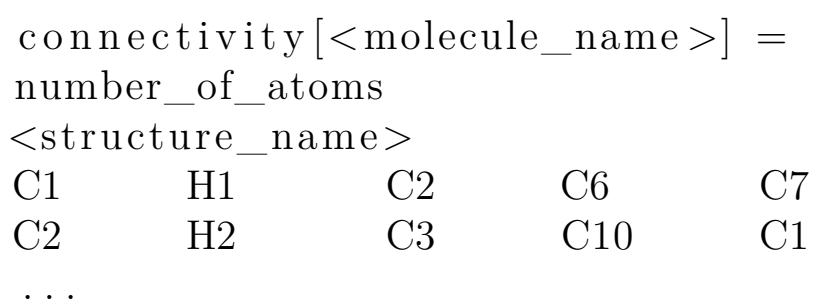

- RDC input: defines the RDCs, their uncertainty and weighting. The respective spin pair is defined by the identifier defined in the structure.

$\mathrm{C} 1 ; \mathrm{H} 1 ; 14.6 ; 0.5 ; 1.0$

$\mathrm{C} 2 ; \mathrm{H} 2 ;-11.0 ; 0.5 ; 1.0$

..

- Keyword input: controls the behavior of TITANIA. Important (case insensitive) keywords are:

- MaxTITANIAiterations: defines the number of iterations TITANIA performes before it is stopped (independent of convergence).

- overoptimizationSteps: defines the number of iterations TITANIA performes after the maximum number of iterations or convergence is reached.

- QfactorConvergence: defines convergence threshold for the rmsd(Q-factors) of two consecutive iteration steps.

- MeanAlignmentConvergence: defines convergence threshold for the $\mathrm{rmsd}\left(\mathbf{S}_{\mathrm{MC}}\right)$ (rmsd of the Saupe tensor elements) of two consecutive iteration steps.

- SigmaAlignmentConvergence: defines convergence threshold for the $r m s d\left(\sigma\left[\mathbf{S}_{\mathrm{MC}}\right]\right)$ ( rmsd of the Saupe tensor element uncertainties) of two consecutive iteration steps.

- MeanAngleConvergence: defines convergence threshold for the $r m s d\left(\mathbf{p}_{\mathrm{MC}}\right)$ (rmsd of the spherical coordinates) of two consecutive iteration steps. 
- SigmaAngleConvergence: defines convergence threshold for the $r m s d\left(\sigma\left[\mathbf{p}_{\mathrm{MC}}\right]\right)$ (rmsd of the spherical coordinate uncertainties) of two consecutive iteration steps.

- SpreadAngleConvergence: defines convergence threshold for the $\operatorname{rmsd}\left(R_{\mathrm{s}}\right)$ (rmsd of the mean RDC vector lengths) of two consecutive iteration steps.

- useRedundantsOnlyAfter defines the number of iterations after which TITANIA only perform redundant internal coordinates structure optimization and skips a prior vector addition step (section 1.3.5).

- useDistances: defines if lower distance bounds are considered in the optimization:

* 0: lower distance bounds are ignored.

* 1: lower distance bounds are fully considered (combination of $\mathbf{2}$ and 3 ).

* 2: lower distance bounds are used in redundant internal coordinates as additional internal coordinate type.

* 3: lower distance bounds are used to force an inversion on atom (groups) if violations are present for consecutive steps.

- redundantsDamping: defines the damping factor $\delta$ (see eq. (26)) for the redundant internal coordinates.

- StaticBondWeighting: defines the static weighting $\left(w_{\text {bond }}\right)$ for bond lengths of redundant internal coordinates (see eq. (25)).

- StaticAngleWeighting: defines the static weighting $\left(w_{\text {angle }}\right)$ for bond angles of redundant internal coordinates (see eq. (25)).

- StaticRDCWeighting: defines the static weighting $\left(w_{\mathrm{RDC}}\right)$ for RDC orientations of redundant internal coordinates (see eq. (25)).

- StaticChiralVolumeWeighting: defines the static weighting $\left(w_{\mathrm{V}_{\mathrm{c}}}\right)$ for chiral volumes of redundant internal coordinates (see eq. (25)).

- StaticDistanceWeighting: defines the static weighting $\left(w_{\text {dist. }}\right)$ for lower distances of redundant internal coordinates (see eq. (25)).

- floatingRDCangles: defines if an angle enclosed by an RDC defined and undefined bond is weighted.

- CalculateFullMatrix: defines how eq. (3b) and eq. (17) are solved:

* 0: solved as a set of vector equations. Enables the use of individual RDC weighting (and even neglecting RDCs).

* 1: solved as full matrix equations. 
- errorWeightInSVD: weights RDCs using their user defined errors. CalculateFullMatrix $=0$ has to be set.

- MonteCarloBootstrapping: defines if a Monte-Carlo bootstrap is performed on every iteration step.

This inputs are gathered in the main input (.tna) file which is used as argument when starting TITANIA. The output file is generated by extending the input file with the respective suffixes (vide infra).

\subsubsection{Output files}

TIANIA has four output files:

- . out contains general informations on the optimization, the input file and keywords. Additionally it contains information on the structure, RDC sampling and final results. Besides this the trajectory of the Q-factors and chiral volumes are reported.

- .out.<setLabel> ali is a output for the individual RDC sets.

- .out.trj contains the full SECONDA analysis (since it is performed on the initial and final structure). In addition it gives information on transformations (initial reference frame and PAS of intertia tensor, change of frames between the iteration steps). The main content are all relevant results obtained from the individual steps:

- Polar angles (with Monte-Carlo results).

- Levenberg-Marquardt parameters.

- Redundant internal coordinate information (including individual damping factors).

- Cartesian coordinates and transformation information.

- Cosine matrix.

- Q-Factors.

- Alignment information (with Monte-Carlo results).

- Dynamics information.

- Stop criteria and the corresponding parameters monitored.

- . out .xyz contains the Cartesian coordinates of the individual iteration steps in the standard xyz-convention. 


\subsection{Mathematics}

\subsubsection{SECONDA weighting scheme}

The covariance matrix used for the SECONDA analysis (see equation 6 in the main text) uses the mean of an individual RDC vector in all media (eq. (1a)) and the weighting factor $w_{\mathrm{m}}$ (eq. (1c)) which uses the mean of all RDCs in one medium (eq. (1b)).

$$
\begin{gathered}
\left\langle D_{\mathrm{k}}\right\rangle=\frac{1}{M} \sum_{m}^{M} D_{\mathrm{k}}^{\mathrm{m}} \\
\left\langle D^{\mathrm{m}}\right\rangle=\frac{1}{K} \sum_{k}^{K} D_{\mathrm{k}}^{\mathrm{m}} \\
w_{\mathrm{m}}=\frac{1}{\sigma_{\mathrm{m}}^{2}}=\frac{1}{\frac{1}{K-1} \sum_{\mathrm{k}}^{\mathrm{K}}\left(D_{\mathrm{k}}^{\mathrm{m}}-\left\langle D^{\mathrm{m}}\right\rangle\right)^{2}}
\end{gathered}
$$

The weighting can in principle be chosen in other ways, but eq. (1c) is the hard coded implementation of TITANIA.

For a proper analysis of the eigenvalues a cutoff of $1 \mathrm{e}-9$ is implemented as numerical threshold.

\subsubsection{RDC theory}

RDCs are defined via the normalized cosine matrix $\boldsymbol{B}$ and alignment matrix $\boldsymbol{A}$, which contain the independent elements of the second-rank alignment tensor $\mathbf{A}$ and the structure related cosine tensor $\mathbf{B}$. These independent elements of a second rank tensor $\mathbf{T}$ are expressed in $\boldsymbol{T}^{(2)}$ by the corresponding normalized elements $T_{\mathrm{ij}}$. ${ }^{[2]}$

$$
\begin{aligned}
\boldsymbol{T}^{(2)} & =\left[T_{\mathrm{zz}}, \frac{1}{\sqrt{3}}\left(T_{\mathrm{xx}}-T_{\mathrm{yy}}\right), \frac{2}{\sqrt{3}} T_{\mathrm{xy}}, \frac{2}{\sqrt{3}} T_{\mathrm{xz}}, \frac{2}{\sqrt{3}} T_{\mathrm{yz}}\right] \\
A_{\mathrm{ij}} & =\left\langle\frac{1}{2}\left(3 \cos \alpha_{\mathrm{i}} \cos \alpha_{\mathrm{j}}-\delta_{\mathrm{ij}}\right)\right\rangle \\
B_{\mathrm{ij}} & =\left\langle\frac{1}{2}\left(3 \cos \beta_{\mathrm{i}} \cos \beta_{\mathrm{j}}-\delta_{\mathrm{ij}}\right)\right\rangle
\end{aligned}
$$

Here $\alpha_{\mathrm{i}}$ is the angles enclosed by the magnetic field vector and the reference axes $(\mathrm{i}=\{x, y, z\})$ of the molecular frame, $\beta_{\mathrm{u}}$ is the angle enclosed by the RDC vector and the reference axes and $\delta_{\mathrm{ij}}$ is the Kronecker symbol. 


$$
\begin{array}{r}
\mathbf{D}_{\mathbf{A}}=\operatorname{diag}\left[D_{\max }\right] \boldsymbol{B} \boldsymbol{A} \\
\left(\boldsymbol{B}^{+} \boldsymbol{B}\right) \boldsymbol{A}=\boldsymbol{B}^{+} \operatorname{diag}\left[D_{\max }^{-1}\right] \mathbf{D}_{\mathbf{A}}
\end{array}
$$

Note that $\mathbf{D}_{\mathbf{A}}$ is the non-normalized RDC matrix, which is the transpose of $\mathbf{D}$ used in equation 4 of the main text. Transposing is not needed but allows for an easier recognition of the orthogonal projector $\left(\boldsymbol{B}^{+} \boldsymbol{B}\right)$ (and later the respective operator $\left.\boldsymbol{F}^{+} \boldsymbol{F}\right) \cdot{ }^{[2]} \operatorname{diag}\left[D_{\max }\right]$ is a matrix containing the maximum possible dipolar coupling for the individual spin pairs on its diagonal. By performing an eigenvalue decomposition on $\mathbf{A}$ the eigenvalue $A_{\mathrm{zz}}$, the rhombicity $R$ as well as the Euler angles $\alpha, \beta$ and $\gamma$ can be calculated. Utilizing the second-rank spherical harmonics $Y_{2, \mathrm{~m}}(\theta, \phi)$, with the spherical coordinates $\theta$ and $\phi$, the general rotations $\mathbf{R}$, using the Wigner elements $D_{\mathrm{M}, \mathrm{m}}^{(2)}$, can be formulated:

$$
\begin{aligned}
& Y_{2,0}^{(2)}(\theta, \phi)=\sqrt{\frac{5}{16 \pi}}\left(3 \cos ^{2}(\theta)-1\right) \\
& Y_{2,1}^{(2)}(\theta, \phi)=-\sqrt{\frac{15}{8 \pi}} \cos (\theta) \sin (\theta) \exp ^{2 \mathrm{i} \phi} \\
& Y_{2,-1}^{(2)}(\theta, \phi)=-Y_{2,1}^{*(2)}(\theta, \phi) \\
& Y_{2,2}^{(2)}(\theta, \phi)=\sqrt{\frac{15}{32 \pi}} \sin ^{2}(\theta) \exp ^{-2 \mathrm{i} \phi} \\
& Y_{2,-2}^{(2)}(\theta, \phi)=Y_{2,2}^{*(2)}(\theta, \phi)
\end{aligned}
$$

$$
\begin{aligned}
\mathbf{R}(\alpha, \beta, \gamma) Y_{2, \mathrm{~m}}^{(2)}(\theta, \phi) & =\sum_{\mathrm{M}=-2}^{2} \exp ^{-\mathrm{i} \alpha \mathrm{M}} d_{\mathrm{M}, \mathrm{m}}^{(2)}(\beta) \exp ^{-\mathrm{i} \gamma \mathrm{m}} Y_{2, \mathrm{M}}^{(2)}(\theta, \phi) \\
& =\sum_{\mathrm{M}=-2}^{2} D_{\mathrm{M}, \mathrm{m}}^{(2)}(\alpha, \beta, \gamma) Y_{2, \mathrm{M}}^{(2)}(\theta, \phi)
\end{aligned}
$$

This allows for the definition of eq. (6b) (which is the static formulation 
of equation 2 in the main text) in any arbitrary reference frame:

$$
\begin{gathered}
D=A_{\mathrm{a}} D_{\max }\left[3 \cos ^{2} \theta^{\mathrm{AF}}-1+\frac{3}{2} R \sin ^{2} \theta^{\mathrm{AF}} \cos 2 \phi^{\mathrm{AF}}\right] \\
D=A_{\mathrm{a}} D_{\max } \sqrt{\frac{4}{5 \pi}}\left[Y_{2,0}^{(2)}+\sqrt{\frac{3}{8}} R\left(Y_{2,2}^{(2)}+Y_{2,2}^{*(2)}\right)\right]
\end{gathered}
$$

The full rotation sequence from the VF to the MF and finally to the AF (eqs. (7) to (11)) for the individual spherical harmonics used in eq. (6b) can be formulated by:

$$
\begin{aligned}
& \mathbf{R}_{\mathrm{VF} \rightarrow \mathrm{MF}}(\alpha, \beta, \gamma) \mathbf{R}_{\mathrm{MF} \rightarrow \mathrm{AF}}(0, \theta, \phi) Y_{2,0}^{(2)}(0,0) \\
&= \mathbf{R}_{\mathrm{VF} \rightarrow \mathrm{MF}}(\alpha, \beta, \gamma) \sum_{\mathrm{M}=-2}^{2} D_{\mathrm{M}, 0}^{(2)}(0, \theta, \phi) Y_{2, \mathrm{M}}^{(2)}(0,0) \\
&= \mathbf{R}_{\mathrm{VF} \rightarrow \mathrm{MF}}(\alpha, \beta, \gamma) D_{0,0}^{(2)}(0, \theta, \phi) \\
&= \sum_{\mathrm{M}=-2}^{2} D_{\mathrm{M}, 0}^{(2)}(\alpha, \beta, \gamma) Y_{2, \mathrm{M}}^{(2)}(\theta, \phi) \\
& \mathbf{R}_{\mathrm{VF} \rightarrow \mathrm{MF}}(\alpha, \beta, \gamma) \mathbf{R}_{\mathrm{MF} \rightarrow \mathrm{AF}}^{(2)}(0, \theta, \phi) Y_{2,2}^{(2)}(0,0) \\
&=\mathbf{R}_{\mathrm{VF} \rightarrow \mathrm{MF}}(\alpha, \beta, \gamma) \sum_{\mathrm{M}=-2}^{2} D_{\mathrm{M}, 2}^{(2)}(0, \theta, \phi) Y_{2, \mathrm{M}}^{(2)}(0,0) \\
&=\mathbf{R}_{\mathrm{VF} \rightarrow \mathrm{MF}}^{(2)}(\alpha, \beta, \gamma) D_{0,2}^{(2)}(0, \theta, \phi) \\
&=\sum_{\mathrm{M}=-2}^{2} D_{\mathrm{M}, 2}^{(2)}(\alpha, \beta, \gamma) Y_{2, \mathrm{M}}^{(2)}(\theta, \phi) \\
& D_{0,2}^{(2)}(0, \theta, \phi)=Y_{2,2}^{(2)}(\theta, \phi)
\end{aligned}
$$




$$
\begin{aligned}
\mathbf{R}_{\mathrm{VF} \rightarrow \mathrm{MF}}(\alpha, \beta, \gamma) \mathbf{R}_{\mathrm{MF} \rightarrow \mathrm{AF}}(0, \theta, \phi) Y_{2,-2}^{(2)}(0,0) \\
=\mathbf{R}_{\mathrm{VF} \rightarrow \mathrm{MF}}(\alpha, \beta, \gamma) \sum_{\mathrm{M}=-2}^{2} D_{\mathrm{M},-2}^{(2)}(0, \theta, \phi) Y_{2, \mathrm{M}}^{(2)}(0,0) \\
=\mathbf{R}_{\mathrm{VF} \rightarrow \mathrm{MF}}(\alpha, \beta, \gamma) D_{0,-2}^{(2)}(0, \theta, \phi) \\
=\sum_{\mathrm{M}=-2}^{2} D_{\mathrm{M},-2}^{(2)}(\alpha, \beta, \gamma) Y_{2, \mathrm{M}}^{(2)}(\theta, \phi) \\
D_{0,-2}^{(2)}(0, \theta, \phi)=Y_{2,-2}^{(2)}(\theta, \phi)
\end{aligned}
$$

Using these rotations the final expression for an RDC in an arbitrary reference frame is obtained:

$$
\begin{aligned}
& D=A_{\mathrm{a}} D_{\max } \sqrt{\frac{4}{5 \pi}}\left[\sum_{\mathrm{M}=-2}^{2}\left\langle D_{\mathrm{M}, 0}^{(2)}\right\rangle\left\langle Y_{2, \mathrm{M}}^{(2)}\right\rangle+\right. \\
& \left.\sqrt{\frac{3}{8}} R\left(\sum_{\mathrm{M}=-2}^{2}\left\langle D_{\mathrm{M}, 2}^{(2)}\right\rangle\left\langle Y_{2, \mathrm{M}}^{(2)}\right\rangle+\sum_{\mathrm{M}=-2}^{2}\left\langle D_{\mathrm{M},-2}^{(2)}\right\rangle\left\langle Y_{2, \mathrm{M}}^{(2)}\right\rangle\right)\right] \\
& D=A_{\mathrm{a}} D_{\max } \sqrt{\frac{4}{5 \pi}} \\
& {\left[\left\langle D_{-2,0}^{(2)}\right\rangle\left\langle Y_{2,-2}^{(2)}\right\rangle+\left\langle D_{-1,0}^{(2)}\right\rangle\left\langle Y_{2,-1}^{(2)}\right\rangle+\left\langle D_{0,0}^{(2)}\right\rangle\left\langle Y_{2,0}^{(2)}\right\rangle+\right.} \\
& \left\langle D_{1,0}^{(2)}\right\rangle\left\langle Y_{2,1}^{(2)}\right\rangle+\left\langle D_{2,0}^{(2)}\right\rangle\left\langle Y_{2,2}^{(2)}\right\rangle+\sqrt{\frac{3}{8}} R \\
& {\left[\left\langle D_{-2,2}^{(2)}\right\rangle\left\langle Y_{2,-2}^{(2)}\right\rangle+\left\langle D_{-1,2}^{(2)}\right\rangle\left\langle Y_{2,-1}^{(2)}\right\rangle+\left\langle D_{0,2}^{(2)}\right\rangle\left\langle Y_{2,0}^{(2)}\right\rangle+\right.} \\
& \left\langle D_{1,2}^{(2)}\right\rangle\left\langle Y_{2,1}^{(2)}\right\rangle+\left\langle D_{2,2}^{(2)}\right\rangle\left\langle Y_{2,2}^{(2)}\right\rangle+ \\
& \left\langle D_{-2,-2}^{(2)}\right\rangle\left\langle Y_{2,-2}^{(2)}\right\rangle+\left\langle D_{-1,-2}^{(2)}\right\rangle\left\langle Y_{2,-1}^{(2)}\right\rangle+\left\langle D_{0,-2}^{(2)}\right\rangle\left\langle Y_{2,0}^{(2)}\right\rangle+ \\
& \left.\left.\left\langle D_{1,-2}^{(2)}\right\rangle\left\langle Y_{2,1}^{(2)}\right\rangle+\left\langle D_{2,-2}^{(2)}\right\rangle\left\langle Y_{2,2}^{(2)}\right\rangle\right]\right] \\
& D=A_{\mathrm{a}} D_{\max } \overrightarrow{\mathbf{F}} \overrightarrow{\mathbf{Y}}
\end{aligned}
$$


where the Wigner rotation elements are combined in $\overrightarrow{\mathbf{F}}$ and the spherical harmonics in $\overrightarrow{\mathbf{Y}}$, respectively.

$$
\begin{aligned}
& \overrightarrow{\mathbf{F}}=\left[\begin{array}{lllll}
F_{-2} & F_{-1} & F_{0} & F_{1} & F_{2}
\end{array}\right] \\
& F_{\mathrm{i}}=D_{\mathrm{i}, 0}^{(2)}+\sqrt{\frac{3}{8}} R\left(D_{\mathrm{i}, 2}^{(2)}+D_{\mathrm{i},-2}^{(2)}\right) \\
& \mathbf{Y}_{\mathrm{ij}}=\left[\begin{array}{c}
Y_{-2,0, \mathrm{ij}} \\
Y_{-1,0, \mathrm{ij}} \\
Y_{0,0, \mathrm{ij}} \\
Y_{1,0, \mathrm{ij}} \\
Y_{2,0, \mathrm{ij}}
\end{array}\right]
\end{aligned}
$$

By normalizing $D$ on the axial component of $\mathbf{A}$ and $D_{\max }$ and extending the dimensions of $D$ the final matrix equation (equation 4 in the main text) is obtained. Note that the resulting matrix $\mathbf{D}_{\mathbf{Y}}$ has the transposed shape of $\mathbf{D}_{\mathbf{A}}$ used in eq. (3). This is not necessary for mathematical correctness but the use of orthogonal projectors ${ }^{[2]}\left(\mathbf{F}^{+} \mathbf{F}\right)$ can be seen easier.

$$
\mathbf{D}_{\mathbf{Y}}=\mathbf{F}\langle\mathbf{Y}\rangle
$$

By SVD of $\mathbf{F}$ refined spherical harmonics $\mathbf{Y}_{\text {ref }}$ are calculated.

$$
\left(\mathbf{F}^{+} \mathbf{F}\right)\left\langle\mathbf{Y}_{\text {ref }}\right\rangle=\mathbf{F}^{+} \mathbf{D}_{\mathbf{Y}}
$$

\subsubsection{Information content of $Y$}

To extract the spherical coordinates $\theta_{\mathrm{av}}$ and $\phi_{\mathrm{av}}$ the Wigner rotations are used to maximize $Y_{2,0}^{(2)}$ in its vector frame (compare figure 2 in main text):

$$
\max \left(Y_{2,0}^{(2)}\left(\theta^{\mathrm{VF}}, \phi^{\mathrm{VF}}\right)\right)=\sum_{\mathrm{M}=-2}^{2} D_{\mathrm{M}, 0}^{(2)}\left(\phi_{\mathrm{av}}^{\mathrm{MF}}, \theta_{\mathrm{av}}^{\mathrm{MF}}, 0\right)\left\langle Y_{2, \mathrm{M}}^{(2)}\left(\theta^{\mathrm{MF}}, \phi^{\mathrm{MF}}\right)\right\rangle
$$

This maximization is performed by using an in-house implementation of the Levenberg-Marquardt algorithm. Additionally the local order parameter of the individual RDCs (see equation 5 in main text), $S_{\text {overall, the asymmetry }}$ parameter $\eta$ (see eq. (20)) and the direction of the asymmetry axis $\varphi$ (see eq. (21)) can be calculated from the spherical harmonics. 


$$
\begin{gathered}
S_{\text {overall }}=\sqrt{\frac{1}{S_{\text {rdc,MFA,max }}^{2}}} \\
\eta=\sqrt{\frac{\sum_{M=-2,2}\left\langle Y_{2, \mathrm{M}}\left(\Theta^{\mathrm{VF}}\right)\right\rangle\left\langle Y_{2, \mathrm{M}}\left(\Theta^{\mathrm{VF}}\right)\right\rangle}{\sum_{M=-2}^{2}\left\langle Y_{2, \mathrm{M}}\left(\Theta^{\mathrm{VF}}\right)\right\rangle\left\langle Y_{2,-\mathrm{M}}\left(\Theta^{\mathrm{VF}}\right)\right\rangle}} \\
\varphi=\frac{1}{2} \operatorname{atan} \frac{\left\langle Y_{2,2}\left(\Theta^{\mathrm{VF}}\right)\right\rangle-\left\langle Y_{2,-2}\left(\Theta^{\mathrm{VF}}\right)\right\rangle}{\mathrm{i}\left(\left\langle Y_{2,2}\left(\Theta^{\mathrm{VF}}\right)\right\rangle+\left\langle Y_{2,-2}\left(\Theta^{\mathrm{VF}}\right)\right\rangle\right)} \\
=\frac{1}{2} \operatorname{atan} \frac{\operatorname{Im}\left(\left\langle Y_{2,2}\left(\Theta^{\mathrm{VF}}\right)\right\rangle\right)}{\operatorname{Re}\left(\left\langle Y_{2,2}\left(\Theta^{\mathrm{VF}}\right)\right\rangle\right)}
\end{gathered}
$$

Here $S_{\text {rdc,MFA,max }}^{2}$ is the largest $S_{\text {rdc,MFA }}^{2}$ directly obtained from the MFA utilizing eq. (17). The spherical coordinates $\theta$ and $\phi$ are combined as tuple in $\Theta$.

\subsubsection{Use of Redundant internal coordinates in TITANIA}

A proper way to represent structures of $\mathrm{N}$ atoms is by using a set of $3 \mathrm{~N}-6$ internal coordinates (which herein are also called holonomic terms). In this case the conversion to Cartesian coordinates can be performed by simple geometric considerations. A more advanced algorithm, implemented in the TITANIA workflow, uses redundant internal coordinates known from quantum chemical computation protocols. In this approach standard internal coordinates, namely bond lengths, bond angles or dihedral angles of more atom tuples than needed to unambiguously define a structure are combined to form an overdetermined representation (more than 3N-6 internal coordinates) of a structure model. As reported in literature, ${ }^{[3,4]}$ the conversion of redundant internal coordinates $q$, containing standard structure parameters (like bond lengths and angles), into Cartesian coordinates is done by the Wilson matrix $\mathbf{B}_{\mathrm{w}}$ :

$$
B_{\mathrm{w}}\left(q_{i}, x_{j}\right)=\frac{\partial q_{i}}{\partial x_{j}}
$$

The change $\Delta \vec{X}$ in Cartesian coordinates $x_{\mathrm{j}}$ (with $\mathrm{j}=\{\mathrm{x}, \mathrm{y}, \mathrm{z}\}$ ) can be calculated utilizing the Moore-Penrose inverse of $\mathbf{B}_{\mathrm{w}}$. For this the deviation between the current and optimal internal coordinates $\left(\Delta q=q_{\mathrm{opt}}-q_{\mathrm{cur}}\right)$ are summarized in the vector $\Delta \overrightarrow{\mathbf{q}}$. This is also called the internal displacement vector. 


$$
\Delta \overrightarrow{\mathrm{X}}=\mathbf{B}_{\mathrm{w}}^{+} \Delta \overrightarrow{\mathbf{q}}
$$

Therefore the iterative optimization of the Cartesian coordinates can be formulated according to:

$$
\overrightarrow{\mathbf{X}}_{\mathbf{i}+\mathbf{1}}=\overrightarrow{\mathrm{X}}_{\mathbf{i}}+\mathbf{B}_{\mathrm{w}}^{+} \Delta \overrightarrow{\mathbf{q}}_{\mathrm{i}}
$$

Since the algorithm is designed to handle small changes (see eq. (22)) its stability can be increased by damping of the individual structure updates (see eq. (24)).

$$
\Delta \overrightarrow{\mathbf{q}}=D \cdot \operatorname{diag}\left[w_{\mathrm{i}}\right] \Delta \overrightarrow{\widetilde{\mathbf{q}}}
$$

Where $w_{\mathrm{i}}$ is the static weighting for the type of $q_{\mathrm{i}}, \Delta \overrightarrow{\widetilde{\mathbf{q}}}$ is the undamped internal displacement vector and $D$ is the global damping factor, which is updated in every step:

$$
D=\frac{\exp \left(3.5 \frac{\text { iter }}{\text { maxiter }}\right)^{2}}{\delta+\exp \left(3.5 \frac{\text { iter }}{\text { maxiter }}\right)^{2}} \frac{\delta+\exp (3.5)^{2}}{\exp (3.5)^{2}}
$$

The course of the function for the damping factors is shown in fig. S-2 for different (user defined) damping constants $\delta$. The factor 3.5 was determined empirically and results in a rather smooth curve of the global damping factor $D$, while allowing for flexible adjustments of it by the user.

Orthogonal projectors $\mathbf{P}$ of the type $\mathbf{P}=\mathbf{B}_{\mathrm{w}} \mathbf{B}_{\mathrm{w}}^{+}$are also used to achieve higher stablity of the algorithm, by projecting the internal displacement vector $\Delta \overrightarrow{\mathbf{q}}$ on the range of $\mathbf{B}_{\mathrm{w}}$. This results in the overall equation for one redundant internal coordinate iteration step:

$$
\overrightarrow{\mathbf{X}}_{\mathbf{i}+\mathbf{1}}=\overrightarrow{\mathbf{X}}_{\mathbf{i}}+\mathbf{B}_{\mathbf{w}}^{+} D \cdot \operatorname{diag}\left[w_{\mathrm{i}}\right]\left(\mathbf{B}_{\mathbf{w}} \mathbf{B}_{\mathbf{w}}^{+} \Delta \overrightarrow{\widetilde{\mathbf{q}}}_{\mathbf{i}}\right)
$$




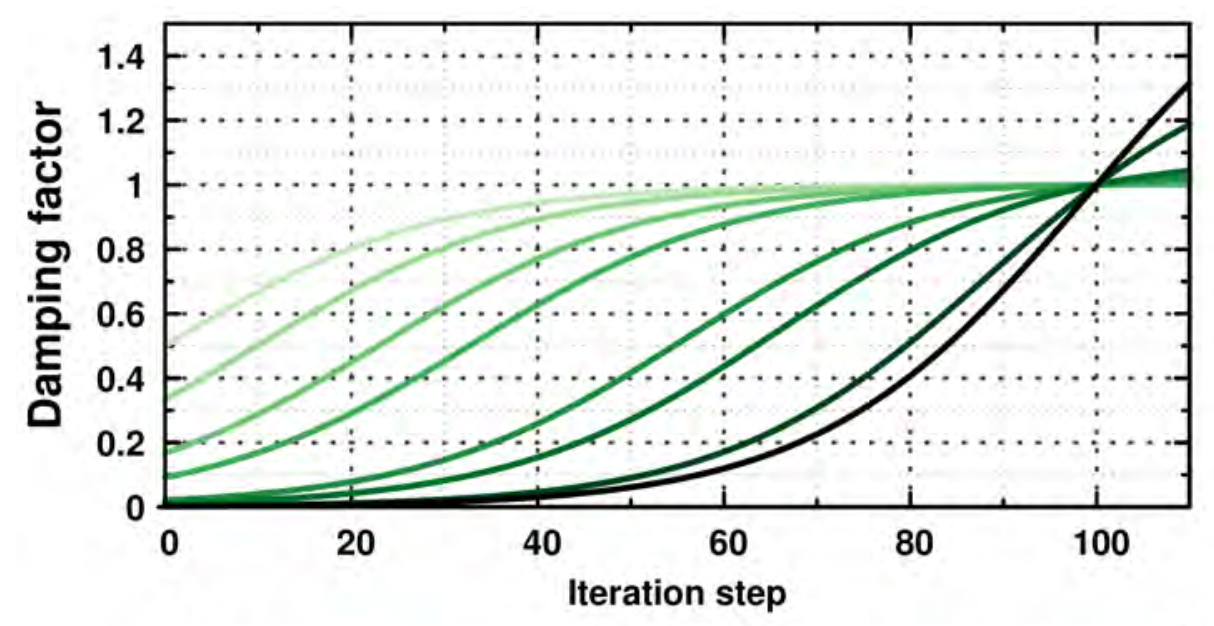

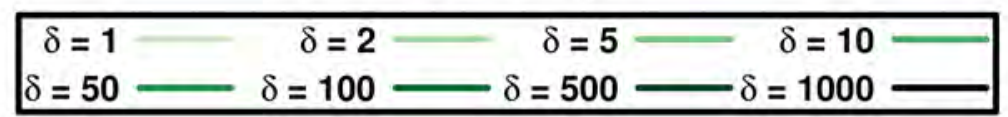

Figure S-2: Damping factors $D$ for a set of damping constants $\delta$. For the calculations a maximum of 100 iterations was assumed.

Care has to be taken when defining the damping constant $\delta$ for an optimization run. Eq (26) is designed to achieve a weighting of 1.0 in the last iteration step. Very large damping constants (approximatly larger than 100) will lead to stronger weighting when defining additional steps (see keyword overoptimizationSteps in section 1.2.1) that can lead to instabilities in the redundant internal coordinates within these additional steps. Another result can be convergence to a local minimum, that cannot be escaped using redundant internal coordinates due to too high damping in the first steps of the TITANIA optimization.

Additionally to the ability to optimize a structure based on an overdetermined set of internal coordinates the algorithm allows for the definition of further terms as restraints. These can (in this context) be RDC-vector orientations or chiral volumes of planar centers to combine the holonomic terms with experimental data. The implementation of RDCs in TITANIA is done by the angle enclosed by $\vec{r}_{i j}(\theta, \phi)$ (determined by the MFA) and the vector $\vec{r}_{i j, c u r}$ calculated from the current structure model. This angle defines a new internal coordinate $q_{\text {cur }}$ which has to be minimized, resulting in $q_{\text {opt }}=0$ and hence $\Delta q=-q_{\text {cur }}$, which is added to the internal displacement vector $\Delta \vec{q}$ 
described above.

\subsubsection{Structures from vector addition}

Alternatively to the default use of redundant internal coordinates TITANIA can use a vector addition algorithm to update structures. It can be very helpful to start an optimization with low maximum iteration steps using this algorithm if redundant internal coordinates do not converge. In this algorithm the non-RDC defined bond vectors (mainly the carbon scaffold) are retained in the initial geometry and only RDC defined bond vectors are updated. This can additionally be combined with the redundant internal coordinate optimization (see keyword useRedundants0nlyAfter in section 1.2.1), where the following algorithm is performed prior to the redundant internal coordinates step.

$$
\begin{aligned}
\overrightarrow{\mathbf{r}}_{\mathrm{RDC}, \mathrm{i}} & =\left\|\overrightarrow{\mathbf{r}}_{\mathrm{i}-1}\right\| \operatorname{sgn}\left(\frac{\overrightarrow{\mathbf{r}}\left(\theta_{\mathrm{i}}, \phi_{\mathrm{i}}\right)^{\mathrm{t}} \cdot \overrightarrow{\mathbf{r}}_{\mathrm{i}-1}}{\left\|\overrightarrow{\mathbf{r}}_{\mathrm{i}-1}\right\|}\right) \overrightarrow{\mathbf{r}}\left(\theta_{\mathrm{i}}, \phi_{\mathrm{i}}\right) \\
\overrightarrow{\mathbf{r}}\left(\theta_{\mathrm{i}}, \phi_{\mathrm{i}}\right) & =\left(\begin{array}{c}
\sin (\theta) \cos (\phi) \\
\sin (\theta) \sin (\phi) \\
\cos (\theta)
\end{array}\right)
\end{aligned}
$$

The resulting vectors are used to update the structure. As first estimation for the correct vector orientation ( $\overrightarrow{\mathbf{r}}_{\mathrm{RDC}}$ vs. $-\overrightarrow{\mathbf{r}}_{\mathrm{RDC}}$ ) the smaller deviation to the previous orientation is used (see sign function in eq. (28)). The bond vectors can be validated and inverted by assessing the mean vector deviation of long range $\mathrm{RDCs}{ }^{\mathrm{n}} D_{\mathrm{HH}}$.

$$
\langle\Delta \overrightarrow{\mathbf{r}}\rangle=\frac{1}{N} \sum_{i}^{N}\left\|\frac{\overrightarrow{\mathbf{r}}_{\mathrm{i}}}{\left\|\overrightarrow{\mathbf{r}}_{\mathrm{i}}\right\|}-\overrightarrow{\mathbf{r}}_{\mathrm{RDC}, \mathrm{i}}\right\|
$$

By this measure the vector orientation is reevaluated. This vector addition algorithm is deactivated by default. The user can activate this algorithm prior to redundant internal coordinates for $\mathrm{N}$ iterations if needed (see above). Positions of atoms which do not contribute to RDC vectors (e.g. hydroxy groups or methyl protons) are updated by a MMFF94 minimization in the course of this algorithm to retain chemically meaningful functional groups / structure moieties. 


\subsubsection{Statistics of directional data}

To estimate $\Delta \theta$ and $\Delta \phi$ (and other uncertainties) a Monte-Carlo bootstrap (with normal distributed sampling in the range of $\Delta D$ ) is used in combination with Gaussian propagation of errors.

$$
\mathbf{D}_{\mathrm{MC}}=\mathbf{D}+\text { rand }_{\text {norm }}[-1.0,1.0] \Delta \mathrm{D}
$$

For the resulting $\mathbf{D}_{\mathrm{MC}}$ matrix equations (3b) and (17) are solved. The uncertainties, which cannot be accessed directly (e.g. spherical coordinates or Euler angles) the Gaussian propagation of errors is employed. For this the general form to estimate the uncertainty for a value $Y$ (not to be confused with the spherical harmonics $\left.Y_{1, \mathrm{~m}}\right)$ with respect to the dependent measurements $x_{\mathrm{i}}$ is used:

$$
\begin{aligned}
\Delta Y & =\sqrt{\sum_{\mathrm{i}}^{\mathrm{m}}\left(\frac{\partial Y}{\partial x_{\mathrm{i}}} \sigma\left(x_{\mathrm{i}}\right)\right)^{2}+2 \sum_{\mathrm{i}}^{\mathrm{m}-1} \sum_{\mathrm{j}=\mathrm{i}+1}^{\mathrm{m}}\left(\frac{\partial Y}{\partial x_{\mathrm{i}}} \frac{\partial Y}{\partial x_{\mathrm{j}}} \operatorname{cov}\left(x_{\mathrm{i}}, x_{\mathrm{j}}\right)\right)} \\
& =\sqrt{\sum_{\mathrm{i}}^{\mathrm{m}} \sum_{\mathrm{j}}^{\mathrm{m}}\left(\frac{\partial Y}{\partial x_{\mathrm{i}}} \frac{\partial Y}{\partial x_{\mathrm{j}}} \operatorname{cov}\left(x_{\mathrm{i}}, x_{\mathrm{j}}\right)\right)}
\end{aligned}
$$

With the covariance cov:

$$
\operatorname{cov}\left(x_{i}, x_{j}\right)=\frac{1}{n} \sum_{k}^{n}\left[\left(\bar{x}_{i}-x_{i, k}\right)\left(\bar{x}_{j}-x_{j, k}\right)\right]
$$

For the Euler angles the numerical gradient is estimated. For the spherical coordinates the analytical gradient is used. Therefore Cartesian vectors representing the orientation of the individual steps are averaged to obtain the mean coordinates:

$$
\begin{aligned}
\bar{X}_{o} & =\frac{1}{n} \sum_{i}^{n} \sin \left(\theta_{i}\right) \cos \left(\phi_{i}\right) \\
\bar{Y}_{o} & =\frac{1}{n} \sum_{i}^{n} \sin \left(\theta_{i}\right) \sin \left(\phi_{i}\right) \\
\bar{Z}_{o} & =\frac{1}{n} \sum_{i}^{n} \cos \left(\theta_{i}\right)
\end{aligned}
$$


The spherical coordinates of the mean vector and its length are accessible by eqs. (34) to (35).

$$
\begin{gathered}
\bar{R}_{s}=\sqrt{\bar{X}_{o}^{2}+\bar{Y}_{o}^{2}+\bar{Z}_{o}^{2}} \\
\bar{\theta}_{\bar{X}, \bar{Y}, \bar{Z}}=\operatorname{acos}\left(\bar{Z}_{o} \bar{R}_{s}^{-1}\right) \\
\bar{\phi}_{\bar{X}, \bar{Y}, \bar{Z}}=\operatorname{atan} 2\left(\bar{Y}_{o} \bar{R}_{s}^{-1}, \bar{X}_{o} \bar{R}_{s}^{-1}\right)
\end{gathered}
$$

The Gaussian propagation of errors for the spherical coordinates $\Delta \theta$ and $\Delta \phi$ can be expressed by:

$$
\begin{aligned}
\Delta \theta & =\sqrt{\sum_{i}^{n} \sum_{j}^{n}\left(\frac{\partial \theta}{\partial x_{i}} \frac{\partial \theta}{\partial x_{j}} \operatorname{cov}\left(x_{i}, x_{j}\right)\right)} \\
\Delta \phi & =\sqrt{\sum_{i}^{n} \sum_{j}^{n}\left(\frac{\partial \phi}{\partial x_{i}} \frac{\partial \phi}{\partial x_{j}} \operatorname{cov}\left(x_{i}, x_{j}\right)\right)}
\end{aligned}
$$

The derivatives in eq. (36) (where $x_{i}=\{x, y, z\}$ ) are:

$$
\begin{aligned}
\frac{\partial \bar{R}_{s}}{\partial x_{i}} & =\frac{x_{i}}{\bar{R}_{s}} \\
\frac{\partial \theta}{\partial x_{i}} & =-\left(\frac{1}{\sqrt{1-\left(\frac{\bar{Z}_{o}}{\bar{R}_{s}}\right)^{2}}}\right)\left(\frac{\frac{\partial \bar{Z}_{o}}{\partial x_{i}} \bar{R}_{s}-\frac{\partial \bar{R}_{s}}{\partial x_{i}} \hat{Z}_{o}}{\bar{R}_{s}^{2}}\right) \\
\frac{\partial \phi}{\partial x_{i}} & =\left(\frac{\frac{\bar{X}_{o}}{\bar{R}_{s}}}{\left(\frac{\bar{X}_{o}}{\bar{R}_{s}}\right)^{2}+\left(\frac{\bar{Y}_{o}}{\bar{R}_{s}}\right)^{2}}\right)\left(\frac{\frac{\partial \bar{Y}_{o}}{\partial x_{i}} \bar{R}_{s}-\frac{\partial \bar{R}_{s}}{\partial x_{i}} \bar{Y}_{o}}{\bar{R}_{s}^{2}}\right) \\
& -\left(\frac{\frac{\bar{Y}_{o}}{\bar{R}_{s}}}{\left(\frac{\bar{X}_{o}}{\bar{R}_{s}}\right)^{2}+\left(\frac{\bar{Y}_{o}}{\bar{R}_{s}}\right)^{2}}\right)\left(\frac{\frac{\partial \bar{X}_{o}}{\partial x_{i}} \bar{R}_{s}-\frac{\partial \bar{R}_{s}}{\partial x_{i}} \bar{X}_{o}}{\bar{R}_{s}^{2}}\right)
\end{aligned}
$$

The spherical variance (eq. (38a)) and spherical standard deviation (eq. (38b)) are calculated as: ${ }^{[5]}$

$$
\begin{aligned}
& \bar{\sigma}_{o}^{2}=1-\bar{R}_{s} \\
& \bar{\sigma}_{o}=\sqrt{-2 \ln \bar{R}_{s}}
\end{aligned}
$$




\section{Isopinocampheol (1)}

Isopinocampheol (IPC) was optimized in six setups, the preparation of which will be briefly explained here. The setup 1-A was generated from 20 randomly generated alignment tensors (refered to as artificial data), to achieve an optimum sampling of the orientations. Two additional setups, to represent experimentally achievable data (1-B) and highly linear dependent data (1C). The orientations are taken from literature, but the number of RDCs was increased. This was done by back-calculation of the full RDC set (referred to as synthetic data). These setups were all calculated from a single rigid structure and alignment tensors, leading to RDCs completly free of errors. Normal distributed random values $\left(\sigma\left({ }^{1} D_{\mathrm{CH}}\right)=0.5 \mathrm{~Hz}, \sigma\left({ }^{1} D_{\mathrm{CC}}\right)=0.125 \mathrm{~Hz}\right.$, $\left.\sigma\left({ }^{n} D_{\mathrm{HH}}\right)=0.25 \mathrm{~Hz}, \mu=0.0\right)$ were added to the RDCs of the setups $\mathbf{1 - A}$ to 1-C to generate the setups $\mathbf{1 - D}$ to $\mathbf{1 - F}$. By this the impact of experimental error on the optimization is investigated.

\subsection{Orientations}

\section{RDC sets}

The alignment parameters of the IPC setups $\mathbf{1 - B}$ and $\mathbf{1 - C}$ were derived from RDCs reported in literature. ${ }^{[6-8]}$ These were used to determine the orientation via SVD. This was done using the in-house RDC module (RDC@hotFCHT) of the hotFCHT software. ${ }^{[9]}$ The recalculation of the orientations might result in different orientations than reported previously due to different weighting of the cosine matrix (to match the TITANIA implementation) and the use of the all-positive Euler angle permutation. 
Table S-1: RDC sets and alignment parameters used for the synthetic RDC sets of run 1-B and 1-C.

\begin{tabular}{l|ccccc} 
Set & Medium & Solvent & Analyte & $T / \mathrm{K}$ & Ref. \\
\hline set 1 & PELG & $\mathrm{CDCl}_{3}$ & $(+)-\mathrm{IPC}$ & 300 & {$[6]$} \\
set 2 & PBDG & $\mathrm{CDCl}_{3}$ & $(+)-\mathrm{IPC}$ & 300 & {$[8]$} \\
set 3 & PPLA & TCE- $d_{2}$ & $(+)-\mathrm{IPC}$ & 300 & {$[7]$} \\
set 4 & PPLA & TCE- $d_{2}$ & $(+)-\mathrm{IPC}$ & 383 & {$[7]$} \\
set 5 & PPDA & TCE- $d_{2}$ & $(+)-\mathrm{IPC}$ & 383 & {$[7]$} \\
set 6 & PPDA & TCE- $d_{2}$ & $(+)-\mathrm{IPC}$ & 300 & {$[7]$} \\
set 7 & PELG & $\mathrm{CDCl}_{3}$ & $(-)-\mathrm{IPC}$ & 300 & {$[6]$} \\
set 8 & PBDG & $\mathrm{CDCl}_{3}$ & $(-)-\mathrm{IPC}$ & 300 & {$[8]$} \\
set 9 & PBLG & $\mathrm{CDCl}_{3}$ & $(+)-\mathrm{IPC}$ & 300 & {$[8]$} \\
set 10 & PBLG & $\mathrm{CDCl}_{3}$ & $(-)-\mathrm{IPC}$ & 300 & {$[8]$}
\end{tabular}

The linear (in)dependence of the respective sets used for the setups 1-B and $\mathbf{1 - C}$ were assessed using the generalized angle $\beta$ :

$$
\begin{gathered}
\cos \beta=\frac{\left\langle\mathbf{A}_{\mathbf{1}} \mid \mathbf{A}_{\mathbf{2}}\right\rangle}{\sqrt{\left\langle\mathbf{A}_{\mathbf{1}} \mid \mathbf{A}_{\mathbf{1}}\right\rangle} \sqrt{\left\langle\mathbf{A}_{\mathbf{2}} \mid \mathbf{A}_{\mathbf{2}}\right\rangle}} \\
\left\langle\mathbf{A}_{\mathbf{1}} \mid \mathbf{A}_{\mathbf{2}}\right\rangle=\operatorname{tr}\left(\mathbf{A}_{\mathbf{1}}^{\mathbf{t}} \cdot \mathbf{A}_{\mathbf{2}}\right)
\end{gathered}
$$




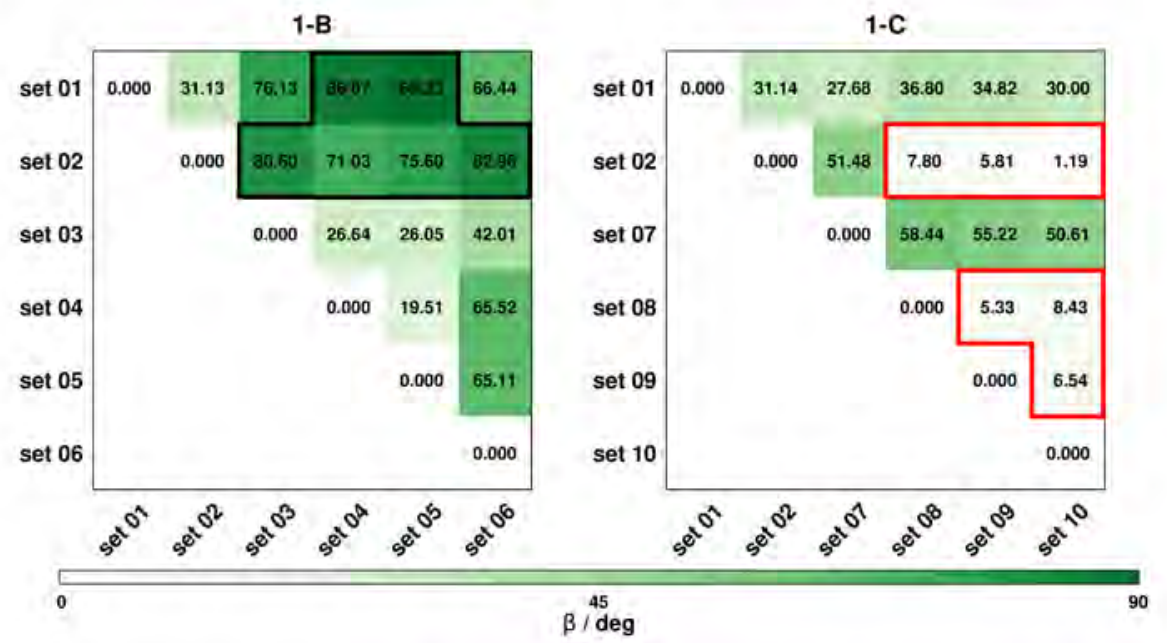

Figure S-3: Pairwise $\beta$ angles of the sets used for setup 1-B and 1-C. The marked fields (black) on the left side show extraordinary large $\beta$ angles. The marks (red) on the right side show the purposefully low angles to ensure linear dependency of the setup. 


\section{2 epi-C3 start structure}

The standard runs were performed with a C3-inverted structure. For runs starting from random coordinates see below (section 2.3) These runs are used to plot the SECONDA data (discussion of $\mathbf{1 - A}$ can be found in the main text) and the change of orientations induced by error. SECONDA is performed on the normalized RDC matrix. The change induced by error is discussed for the epi-C3 structure only, since the random coordinates were used for the error free data only. The setups $\mathbf{1 - D}$ to $\mathbf{1 - F}$ were additionally optimized using vector addition. The change induced by error (see section 2.2.5) is not discussed for the vector addition algorithm as a bias would be added due to the change of the structure generation algorithm. 


\subsubsection{SECONDA plots}

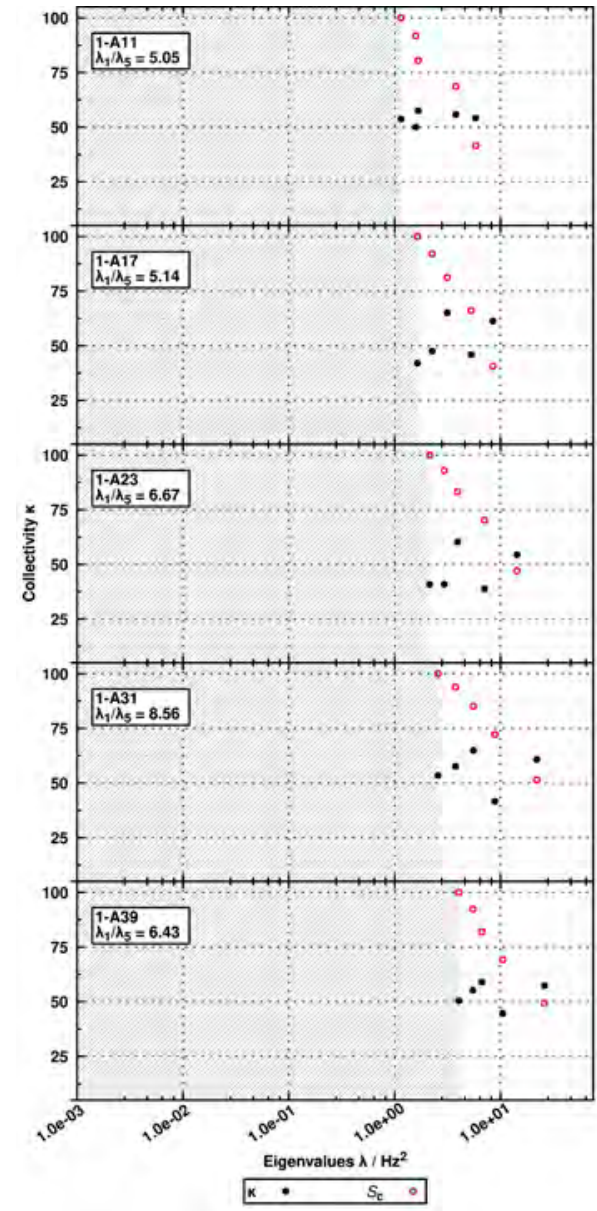

Figure S-4: SECONDA plot of the individual runs in setup 1-A.

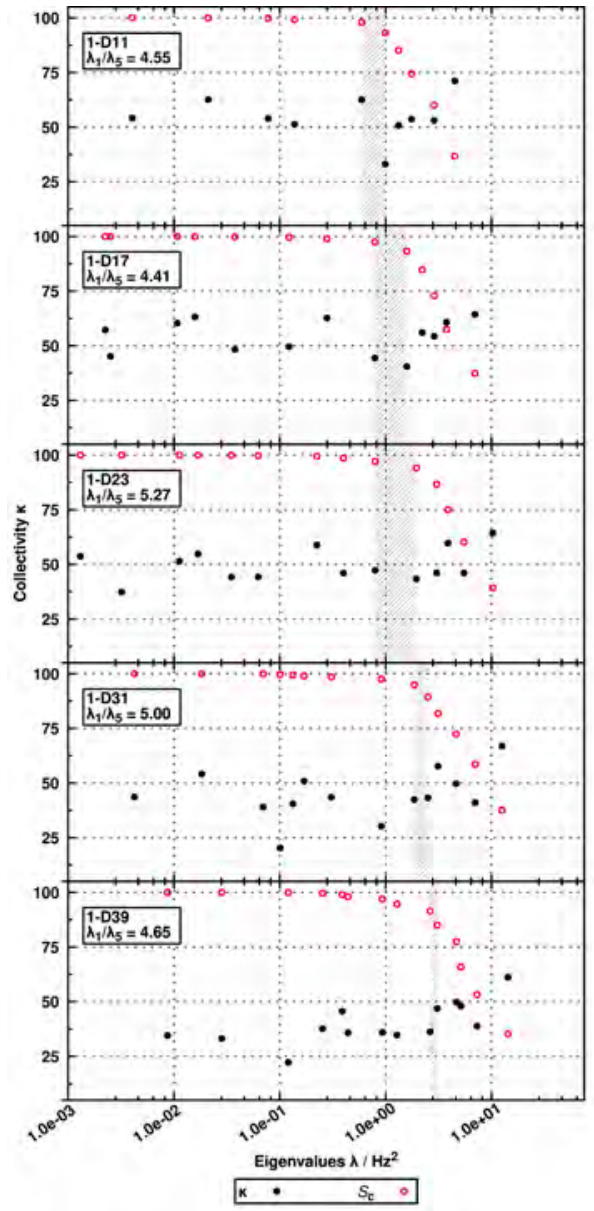

Figure S-5: SECONDA plot of the individual runs in setup 1-D. 


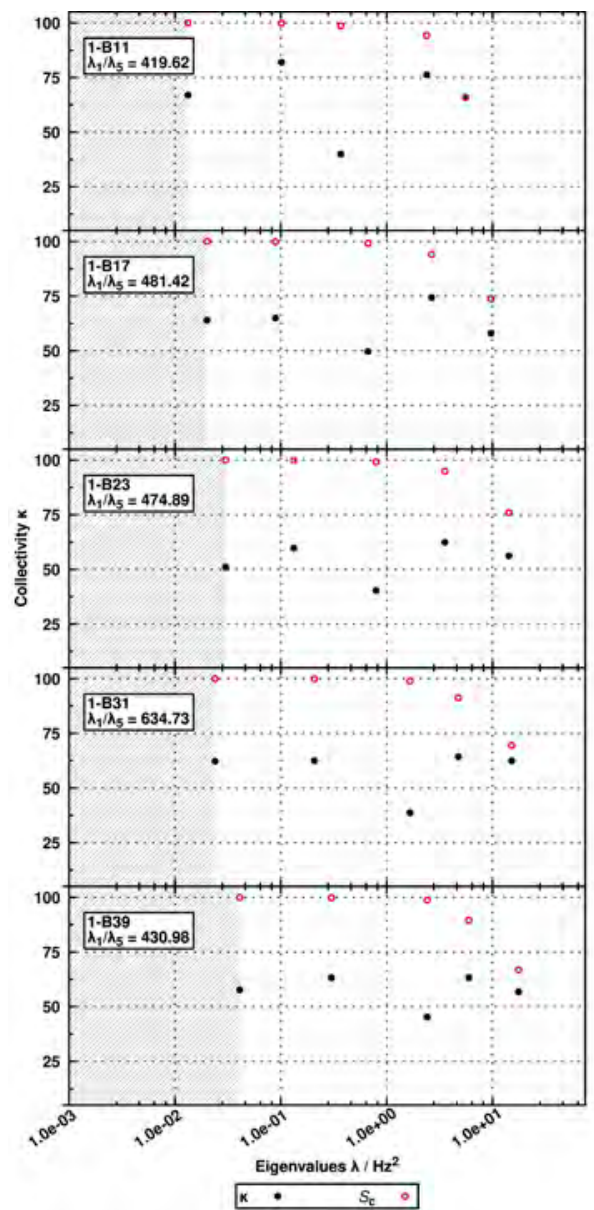

Figure S-6: SECONDA plot of the individual runs in setup 1-B.

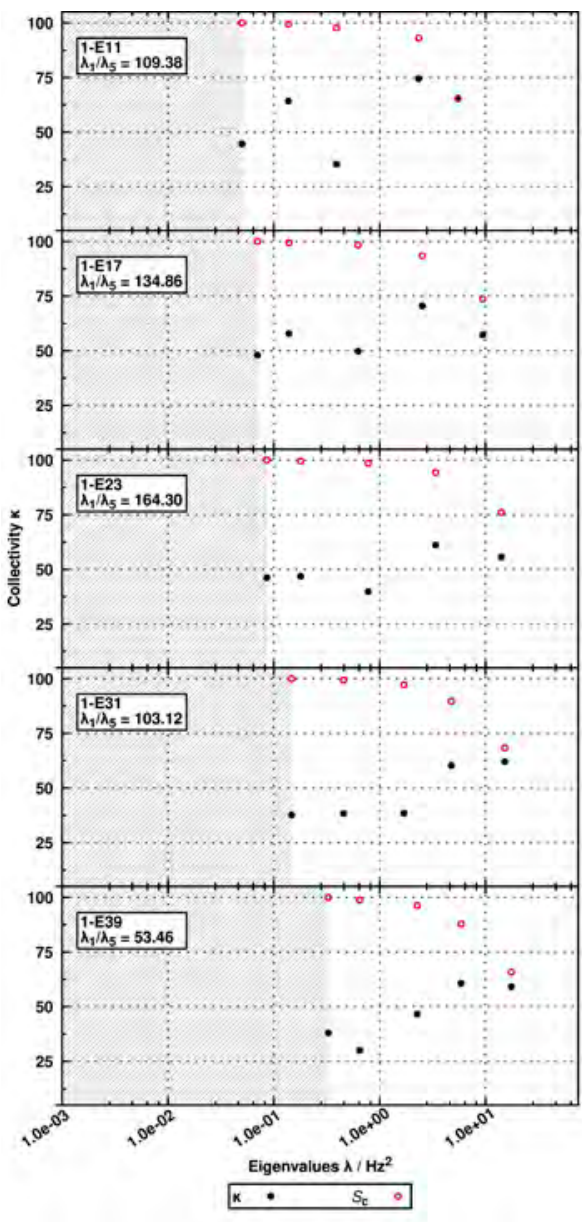

Figure S-7: SECONDA plot of the individual runs in setup 1-E. 


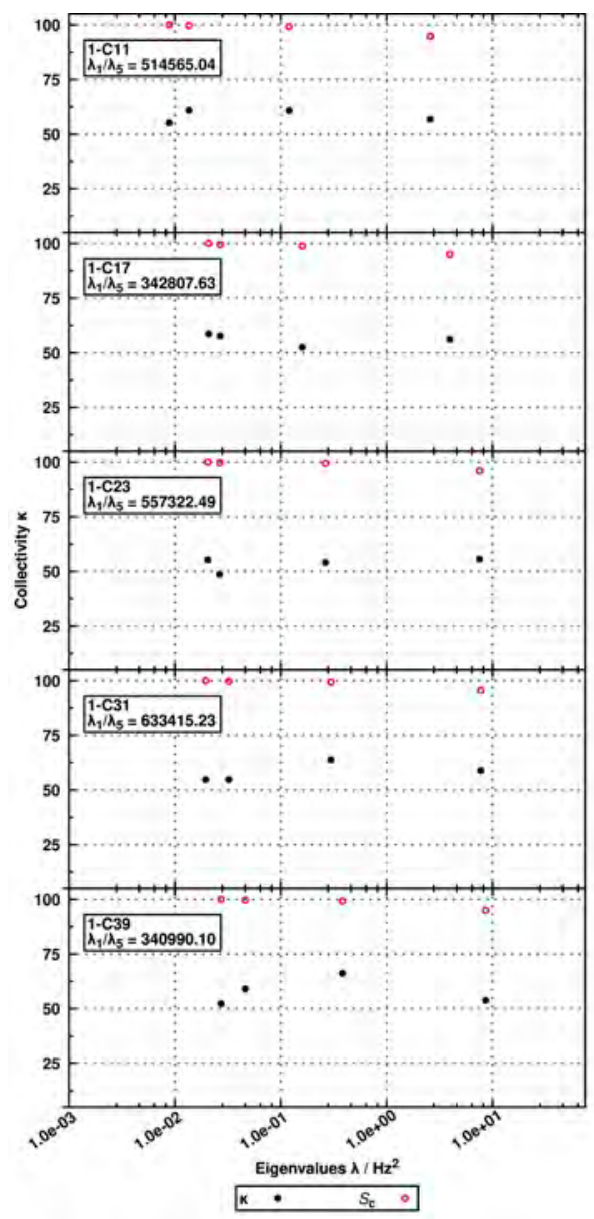

Figure S-8: SECONDA plot of the individual runs in setup 1-C.

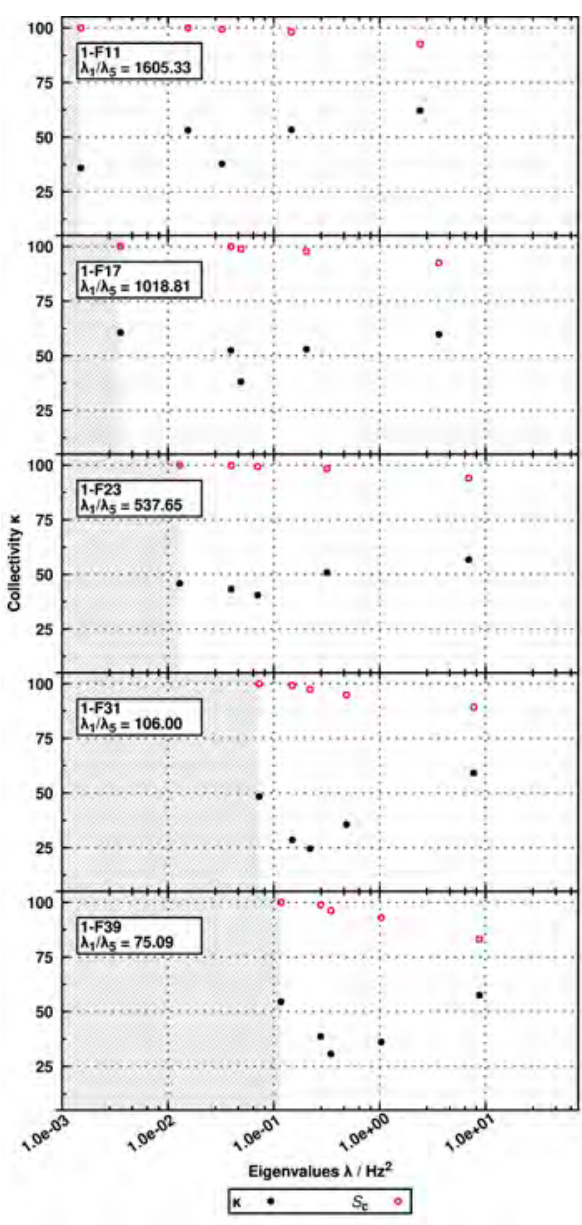

Figure S-9: SECONDA plot of the individual runs in setup 1-F. 
The SECONDA plots give insight into the RDC information content of the setups 1-A to 1-F. In the main text we focused on the assessment of the linear independence of the setups. Here we investigate the impact of synthetic heterogeneity (in this case error, added via normal distributed random numbers) on the error free setups $\mathbf{1 - A}$ to $\mathbf{1 - C}$, by comparing these to the respective setups $\mathbf{1 - D}$ to $\mathbf{1 - F}$. Hus and Brüschweiler have shown earlier that the addition of heterogeneity to a back-calculated RDC matrix of full rank has little impact on the principle variances $\lambda_{1}$ to $\lambda_{5}{ }^{[10]}$

This result can as well be found in the comparison of fig. S-4 and S-5 (comparison of $\mathbf{1 - A}$ and $\mathbf{1 - D}$ ). Here the eigenvalues $\lambda_{1}$ to $\lambda_{5}$ are nearly unchanged by the Gaussian random numbers. As expected for 1-D additional eigenvalues $\lambda_{n}(n>5)$ become non-zero. This is typical for RDC matrices with heterogeneity. The high number of additional eigenvalues can be explained by the large relative errors (see table S-41).

The setups 1-B and 1-E show the above behavior for the first three principle variances. The eigenvalues $\lambda_{4}$ and $\lambda_{5}$ in contrast already show a slight dependence on the heterogeneity. These are the same eigenvalues which had a contribution of less than $1.0 \%$ to the overall variance for these setups (see threshold discussed in main text).

This, in combination with the rather large $\lambda_{1} / \lambda_{5}$ ratio, implies that no complete linear independence of five orientations is achieved for the setup. In contrast to 1-D no additional non-zero eigenvalues $\left(\lambda_{6}<1 e-9\right.$ for all runs of 1-E) are found. To the best of our knowledge no (published) investigation of synthetic RDC data containing only 6 sets, to which synthetic errors were added, exists. In the setup 1-B, 1-C, 1-E and $\mathbf{1 - F}$ we used the minimum number of RDC sets needed to allow for a detection of heterogeneity (The rank of an $n \times m$ matrix cannot exceed $n$ if $n<m$ or $m$ if $m<n$, respectively. Therefore an RDC matrix of 5 media can only achieve a maximum rank of 5.). It is thus assumed that the minimum number of RDC sets in combination with the mediocre sampling of the orientations was not sufficient to detect the heterogeneity.

This behavior is even more pronounced for the setups 1-C and 1-F. Additionally to not showing a non-zero eigenvalue $\lambda_{6}$ when adding synthetic error, 1-C does not show $\lambda_{5}$ within the scale used in the plot $\left(\lambda_{5}<1 e-3\right)$. For the sets with 11 to 23 RDCs the principle variances $\lambda_{1}$ and $\lambda_{2}$ only show slight dependence on the heterogeneity. For the last two setups 1-C31 and 1-C39 this is only true for $\lambda_{1}$. This behavior coincides with the high linear dependence desired for the setup 1-C. Again the values dependent on the heterogeneity have less than $1.0 \%$ contribution to the overall variance as discussed in the main text. 


\subsubsection{Orientations}

\section{1-A}

\section{Input orientations}

The RDCs of setup 1-A were calculated by the orientations summarized in table S-2.

Table S-2: Orientational data used for the synthetic RDC sets of setup 1-A and 1-D.

\begin{tabular}{l|ccrrr} 
Set & $\boldsymbol{A}_{\text {zz }}$ & \multicolumn{1}{c}{$\boldsymbol{\alpha}$} & \multicolumn{1}{c}{$\boldsymbol{\alpha} /^{\circ}$} & \multicolumn{1}{c}{$\boldsymbol{\beta} /{ }^{\circ}$} & \multicolumn{1}{c}{$\boldsymbol{\gamma} /{ }^{\circ}$} \\
\hline set 1 & $6.502 \mathrm{e}-04$ & $2.810 \mathrm{e}-01$ & 8.144 & 141.340 & 82.889 \\
set 2 & $8.426 \mathrm{e}-05$ & $2.222 \mathrm{e}-01$ & 145.757 & 110.718 & 24.512 \\
set 3 & $8.788 \mathrm{e}-05$ & $3.201 \mathrm{e}-01$ & 7.168 & 99.611 & 155.036 \\
set 4 & $4.350 \mathrm{e}-04$ & $5.409 \mathrm{e}-01$ & 100.047 & 51.633 & 116.928 \\
set 5 & $3.411 \mathrm{e}-04$ & $3.893 \mathrm{e}-01$ & 140.036 & 73.152 & 84.947 \\
set 6 & $8.659 \mathrm{e}-04$ & $4.858 \mathrm{e}-01$ & 165.509 & 48.826 & 27.761 \\
set 7 & $8.074 \mathrm{e}-04$ & $7.404 \mathrm{e}-03$ & 44.055 & 123.341 & 119.405 \\
set 8 & $4.177 \mathrm{e}-04$ & $2.348 \mathrm{e}-01$ & 87.834 & 54.652 & 157.368 \\
set 9 & $4.881 \mathrm{e}-05$ & $2.929 \mathrm{e}-01$ & 111.992 & 33.749 & 60.348 \\
set 10 & $7.993 \mathrm{e}-04$ & $5.760 \mathrm{e}-01$ & 12.000 & 148.761 & 72.686 \\
set 11 & $3.255 \mathrm{e}-04$ & $1.066 \mathrm{e}-01$ & 90.282 & 22.718 & 122.776 \\
set 12 & $4.468 \mathrm{e}-04$ & $4.043 \mathrm{e}-01$ & 9.639 & 62.911 & 98.912 \\
set 13 & $3.196 \mathrm{e}-04$ & $1.107 \mathrm{e}-01$ & 82.100 & 168.690 & 6.311 \\
set 14 & $6.600 \mathrm{e}-05$ & $2.687 \mathrm{e}-01$ & 107.921 & 153.048 & 77.132 \\
set 15 & $7.857 \mathrm{e}-04$ & $2.918 \mathrm{e}-02$ & 69.136 & 138.490 & 101.059 \\
set 16 & $4.146 \mathrm{e}-04$ & $4.251 \mathrm{e}-01$ & 30.733 & 107.546 & 178.566 \\
set 17 & $6.562 \mathrm{e}-04$ & $5.205 \mathrm{e}-01$ & 89.664 & 38.741 & 107.788 \\
set 18 & $5.977 \mathrm{e}-04$ & $4.170 \mathrm{e}-01$ & 54.711 & 118.926 & 144.841 \\
set 19 & $8.809 \mathrm{e}-04$ & $1.118 \mathrm{e}-01$ & 63.241 & 75.212 & 163.185 \\
set 20 & $6.492 \mathrm{e}-04$ & $6.360 \mathrm{e}-01$ & 117.915 & 146.575 & 88.796
\end{tabular}

\section{Output orientations}

All orientiations of setup 1-A (11-39) obtained from the full TITANIA optimization runs are listed in the following section. Changes of the Euler angles compared to the data listed in table S-2 are due to the change of the reference frames (TITANIA used the principle axis system of the molecule) and changes in the structure. The comparison of the input and output data is summarized in table $\mathrm{S}-35$ (section 2.2.4). 
Table S-3: Orientational data of IPC 1 obtained by TITANIA on the last optimization iteration for the run 1-A11.

\begin{tabular}{l|ccrrr} 
Set & $\boldsymbol{A}_{\mathrm{zz}}$ & $\boldsymbol{R}$ & \multicolumn{1}{c}{$\boldsymbol{\alpha} /{ }^{\circ}$} & \multicolumn{1}{c}{$\boldsymbol{\beta} /{ }^{\circ}$} & \multicolumn{1}{c}{$\boldsymbol{\gamma} /{ }^{\circ}$} \\
\hline set 1 & $6.987 \mathrm{e}-04$ & $2.391 \mathrm{e}-01$ & 173.230 & 35.413 & 98.063 \\
set 2 & $8.006 \mathrm{e}-05$ & $3.196 \mathrm{e}-01$ & 148.225 & 72.877 & 149.183 \\
set 3 & $8.250 \mathrm{e}-05$ & $3.865 \mathrm{e}-01$ & 9.152 & 78.483 & 19.577 \\
set 4 & $4.395 \mathrm{e}-04$ & $5.370 \mathrm{e}-01$ & 91.183 & 122.082 & 58.114 \\
set 5 & $3.324 \mathrm{e}-04$ & $2.391 \mathrm{e}-01$ & 121.320 & 105.117 & 85.795 \\
set 6 & $8.935 \mathrm{e}-04$ & $5.056 \mathrm{e}-01$ & 170.915 & 126.443 & 142.591 \\
set 7 & $8.092 \mathrm{e}-04$ & $1.625 \mathrm{e}-01$ & 158.125 & 52.441 & 55.599 \\
set 8 & $4.153 \mathrm{e}-04$ & $2.421 \mathrm{e}-01$ & 83.303 & 121.542 & 21.879 \\
set 9 & $4.413 \mathrm{e}-05$ & $2.669 \mathrm{e}-01$ & 120.063 & 140.779 & 114.967 \\
set 10 & $8.821 \mathrm{e}-04$ & $5.122 \mathrm{e}-01$ & 2.805 & 31.745 & 110.056 \\
set 11 & $2.943 \mathrm{e}-04$ & $2.059 \mathrm{e}-01$ & 100.496 & 153.250 & 47.404 \\
set 12 & $4.430 \mathrm{e}-04$ & $3.750 \mathrm{e}-01$ & 15.503 & 110.386 & 71.077 \\
set 13 & $3.267 \mathrm{e}-04$ & $1.696 \mathrm{e}-01$ & 96.550 & 16.491 & 168.600 \\
set 14 & $7.347 \mathrm{e}-05$ & $2.476 \mathrm{e}-01$ & 101.082 & 25.566 & 111.816 \\
set 15 & $8.259 \mathrm{e}-04$ & $8.799 \mathrm{e}-02$ & 135.239 & 36.597 & 79.886 \\
set 16 & $4.041 \mathrm{e}-04$ & $3.886 \mathrm{e}-01$ & 144.814 & 111.189 & 171.088 \\
set 17 & $5.990 \mathrm{e}-04$ & $6.274 \mathrm{e}-01$ & 90.037 & 135.426 & 69.611 \\
set 18 & $5.901 \mathrm{e}-04$ & $3.496 \mathrm{e}-01$ & 51.474 & 57.966 & 30.026 \\
set 19 & $8.517 \mathrm{e}-04$ & $2.136 \mathrm{e}-01$ & 53.130 & 101.840 & 13.466 \\
set 20 & $7.067 \mathrm{e}-04$ & $6.281 \mathrm{e}-01$ & 108.920 & 28.612 & 100.249
\end{tabular}


Table S-4: Orientational data of IPC 1 obtained by TITANIA on the last optimization iteration of run 1-A17.

\begin{tabular}{l|ccrrr} 
Set & $\boldsymbol{A}_{\mathrm{zz}}$ & $\boldsymbol{R}$ & \multicolumn{1}{c}{$\boldsymbol{\alpha} /{ }^{\circ}$} & \multicolumn{1}{c}{$\boldsymbol{\beta} /{ }^{\circ}$} & \multicolumn{1}{c}{$\boldsymbol{\gamma} /{ }^{\circ}$} \\
\hline set 1 & $5.996 \mathrm{e}-04$ & $2.893 \mathrm{e}-01$ & 4.063 & 33.244 & 89.880 \\
set 2 & $8.144 \mathrm{e}-05$ & $2.329 \mathrm{e}-01$ & 143.398 & 65.458 & 150.476 \\
set 3 & $8.304 \mathrm{e}-05$ & $3.081 \mathrm{e}-01$ & 10.468 & 78.033 & 18.078 \\
set 4 & $3.899 \mathrm{e}-04$ & $5.990 \mathrm{e}-01$ & 104.113 & 125.515 & 58.970 \\
set 5 & $3.217 \mathrm{e}-04$ & $4.017 \mathrm{e}-01$ & 139.266 & 101.826 & 89.719 \\
set 6 & $8.305 \mathrm{e}-04$ & $4.677 \mathrm{e}-01$ & 159.538 & 127.394 & 142.928 \\
set 7 & $7.815 \mathrm{e}-04$ & $3.050 \mathrm{e}-02$ & 18.681 & 54.198 & 51.713 \\
set 8 & $3.877 \mathrm{e}-04$ & $2.212 \mathrm{e}-01$ & 93.107 & 125.785 & 17.000 \\
set 9 & $4.655 \mathrm{e}-05$ & $3.118 \mathrm{e}-01$ & 110.950 & 140.973 & 113.043 \\
set 10 & $7.367 \mathrm{e}-04$ & $5.681 \mathrm{e}-01$ & 8.403 & 25.069 & 102.677 \\
set 11 & $3.118 \mathrm{e}-04$ & $1.368 \mathrm{e}-01$ & 93.194 & 154.583 & 57.714 \\
set 12 & $4.167 \mathrm{e}-04$ & $3.884 \mathrm{e}-01$ & 13.190 & 112.505 & 75.969 \\
set 13 & $3.121 \mathrm{e}-04$ & $8.711 \mathrm{e}-02$ & 129.026 & 169.064 & 13.957 \\
set 14 & $6.307 \mathrm{e}-05$ & $2.912 \mathrm{e}-01$ & 109.675 & 21.197 & 97.510 \\
set 15 & $7.344 \mathrm{e}-04$ & $2.431 \mathrm{e}-02$ & 163.417 & 37.521 & 69.041 \\
set 16 & $3.938 \mathrm{e}-04$ & $3.937 \mathrm{e}-01$ & 143.396 & 107.184 & 173.467 \\
set 17 & $6.030 \mathrm{e}-04$ & $5.700 \mathrm{e}-01$ & 94.811 & 137.993 & 69.793 \\
set 18 & $5.850 \mathrm{e}-04$ & $4.410 \mathrm{e}-01$ & 59.823 & 59.975 & 27.285 \\
set 19 & $8.042 \mathrm{e}-04$ & $7.467 \mathrm{e}-02$ & 72.594 & 104.486 & 10.478 \\
set 20 & $6.241 \mathrm{e}-04$ & $6.500 \mathrm{e}-01$ & 121.371 & 28.026 & 83.300
\end{tabular}


Table S-5: Orientational data of IPC 1 obtained by TITANIA on the last optimization iteration of run 1-A23.

\begin{tabular}{l|ccrrr} 
Set & $\boldsymbol{A}_{\mathrm{zz}}$ & $\boldsymbol{R}$ & \multicolumn{1}{c}{$\boldsymbol{\alpha} /{ }^{\circ}$} & \multicolumn{1}{c}{$\boldsymbol{\beta} /{ }^{\circ}$} & \multicolumn{1}{c}{$\boldsymbol{\gamma} /{ }^{\circ}$} \\
\hline set 1 & $6.169 \mathrm{e}-04$ & $2.594 \mathrm{e}-01$ & 10.075 & 29.966 & 89.231 \\
set 2 & $8.209 \mathrm{e}-05$ & $2.349 \mathrm{e}-01$ & 138.138 & 62.814 & 154.005 \\
set 3 & $8.056 \mathrm{e}-05$ & $3.130 \mathrm{e}-01$ & 15.740 & 79.008 & 19.822 \\
set 4 & $4.053 \mathrm{e}-04$ & $5.708 \mathrm{e}-01$ & 106.846 & 122.819 & 63.396 \\
set 5 & $3.164 \mathrm{e}-04$ & $3.903 \mathrm{e}-01$ & 139.577 & 98.677 & 91.613 \\
set 6 & $8.379 \mathrm{e}-04$ & $4.699 \mathrm{e}-01$ & 156.880 & 124.592 & 143.713 \\
set 7 & $7.870 \mathrm{e}-04$ & $1.140 \mathrm{e}-02$ & 25.731 & 51.631 & 51.858 \\
set 8 & $3.923 \mathrm{e}-04$ & $2.540 \mathrm{e}-01$ & 97.656 & 125.724 & 23.552 \\
set 9 & $4.746 \mathrm{e}-05$ & $3.121 \mathrm{e}-01$ & 109.038 & 137.538 & 115.013 \\
set 10 & $7.529 \mathrm{e}-04$ & $5.458 \mathrm{e}-01$ & 13.058 & 21.762 & 102.001 \\
set 11 & $3.142 \mathrm{e}-04$ & $1.186 \mathrm{e}-01$ & 100.494 & 151.715 & 67.139 \\
set 12 & $4.166 \mathrm{e}-04$ & $4.047 \mathrm{e}-01$ & 16.801 & 109.722 & 78.695 \\
set 13 & $3.105 \mathrm{e}-04$ & $1.000 \mathrm{e}-01$ & 148.187 & 169.372 & 36.946 \\
set 14 & $6.393 \mathrm{e}-05$ & $2.737 \mathrm{e}-01$ & 112.892 & 18.225 & 95.869 \\
set 15 & $7.556 \mathrm{e}-04$ & $1.102 \mathrm{e}-02$ & 108.974 & 34.654 & 68.109 \\
set 16 & $3.866 \mathrm{e}-04$ & $4.241 \mathrm{e}-01$ & 139.306 & 105.166 & 174.307 \\
set 17 & $6.235 \mathrm{e}-04$ & $5.474 \mathrm{e}-01$ & 97.284 & 134.667 & 74.761 \\
set 18 & $5.779 \mathrm{e}-04$ & $4.344 \mathrm{e}-01$ & 64.341 & 59.185 & 27.119 \\
set 19 & $8.060 \mathrm{e}-04$ & $1.201 \mathrm{e}-01$ & 77.509 & 105.948 & 14.444 \\
set 20 & $6.347 \mathrm{e}-04$ & $6.223 \mathrm{e}-01$ & 124.753 & 25.287 & 82.279
\end{tabular}


Table S-6: Orientational data of IPC 1 obtained by TITANIA on the last optimization iteration of run 1-A31.

\begin{tabular}{l|ccrrr} 
Set & $\boldsymbol{A}_{\mathrm{zz}}$ & $\boldsymbol{R}$ & \multicolumn{1}{c}{$\boldsymbol{\alpha} /{ }^{\circ}$} & \multicolumn{1}{c}{$\boldsymbol{\beta} /{ }^{\circ}$} & \multicolumn{1}{c}{$\boldsymbol{\gamma} /{ }^{\circ}$} \\
\hline set 1 & $6.190 \mathrm{e}-04$ & $2.811 \mathrm{e}-01$ & 13.017 & 30.870 & 89.129 \\
set 2 & $7.992 \mathrm{e}-05$ & $2.262 \mathrm{e}-01$ & 139.312 & 62.830 & 154.086 \\
set 3 & $8.364 \mathrm{e}-05$ & $3.134 \mathrm{e}-01$ & 15.105 & 80.683 & 20.210 \\
set 4 & $4.125 \mathrm{e}-04$ & $5.521 \mathrm{e}-01$ & 108.291 & 123.393 & 65.004 \\
set 5 & $3.227 \mathrm{e}-04$ & $3.929 \mathrm{e}-01$ & 142.675 & 99.528 & 92.638 \\
set 6 & $8.156 \mathrm{e}-04$ & $4.773 \mathrm{e}-01$ & 159.481 & 124.356 & 145.477 \\
set 7 & $7.676 \mathrm{e}-04$ & $2.125 \mathrm{e}-03$ & 126.045 & 52.395 & 51.820 \\
set 8 & $3.918 \mathrm{e}-04$ & $2.516 \mathrm{e}-01$ & 97.559 & 125.949 & 25.152 \\
set 9 & $4.639 \mathrm{e}-05$ & $2.922 \mathrm{e}-01$ & 110.659 & 138.006 & 115.806 \\
set 10 & $7.618 \mathrm{e}-04$ & $5.721 \mathrm{e}-01$ & 15.238 & 22.999 & 100.518 \\
set 11 & $3.107 \mathrm{e}-04$ & $1.059 \mathrm{e}-01$ & 110.160 & 152.268 & 68.564 \\
set 12 & $4.223 \mathrm{e}-04$ & $3.985 \mathrm{e}-01$ & 15.312 & 110.826 & 79.999 \\
set 13 & $3.054 \mathrm{e}-04$ & $1.247 \mathrm{e}-01$ & 141.816 & 170.547 & 36.014 \\
set 14 & $6.320 \mathrm{e}-05$ & $2.628 \mathrm{e}-01$ & 115.528 & 19.124 & 93.566 \\
set 15 & $7.486 \mathrm{e}-04$ & $2.382 \mathrm{e}-02$ & 84.084 & 35.278 & 68.484 \\
set 16 & $3.946 \mathrm{e}-04$ & $4.158 \mathrm{e}-01$ & 141.338 & 103.733 & 176.264 \\
set 17 & $6.258 \mathrm{e}-04$ & $5.190 \mathrm{e}-01$ & 98.737 & 135.059 & 75.673 \\
set 18 & $5.745 \mathrm{e}-04$ & $4.025 \mathrm{e}-01$ & 64.180 & 60.459 & 26.946 \\
set 19 & $8.290 \mathrm{e}-04$ & $1.175 \mathrm{e}-01$ & 73.393 & 106.150 & 15.491 \\
set 20 & $6.221 \mathrm{e}-04$ & $6.258 \mathrm{e}-01$ & 126.260 & 26.241 & 80.907
\end{tabular}


Table S-7: Orientational data of IPC 1 obtained by TITANIA on the last optimization iteration of run 1-A39.

\begin{tabular}{l|ccrrr} 
Set & $\boldsymbol{A}_{\mathrm{zz}}$ & $\boldsymbol{R}$ & \multicolumn{1}{c}{$\boldsymbol{\alpha} /{ }^{\circ}$} & \multicolumn{1}{c}{$\boldsymbol{\beta} /{ }^{\circ}$} & \multicolumn{1}{c}{$\boldsymbol{\gamma} /{ }^{\circ}$} \\
\hline set 1 & $6.214 \mathrm{e}-04$ & $2.645 \mathrm{e}-01$ & 13.041 & 30.120 & 90.406 \\
set 2 & $7.904 \mathrm{e}-05$ & $2.375 \mathrm{e}-01$ & 138.195 & 62.436 & 154.814 \\
set 3 & $8.340 \mathrm{e}-05$ & $3.220 \mathrm{e}-01$ & 16.744 & 79.902 & 20.496 \\
set 4 & $4.130 \mathrm{e}-04$ & $5.483 \mathrm{e}-01$ & 107.672 & 122.359 & 64.690 \\
set 5 & $3.220 \mathrm{e}-04$ & $3.914 \mathrm{e}-01$ & 141.730 & 98.213 & 92.767 \\
set 6 & $8.254 \mathrm{e}-04$ & $4.833 \mathrm{e}-01$ & 157.970 & 124.428 & 144.468 \\
set 7 & $7.727 \mathrm{e}-04$ & $1.184 \mathrm{e}-02$ & 103.052 & 51.495 & 51.989 \\
set 8 & $3.957 \mathrm{e}-04$ & $2.374 \mathrm{e}-01$ & 98.850 & 125.046 & 26.086 \\
set 9 & $4.671 \mathrm{e}-05$ & $3.037 \mathrm{e}-01$ & 110.708 & 137.287 & 115.432 \\
set 10 & $7.640 \mathrm{e}-04$ & $5.643 \mathrm{e}-01$ & 13.978 & 22.442 & 102.834 \\
set 11 & $3.104 \mathrm{e}-04$ & $1.169 \mathrm{e}-01$ & 103.444 & 151.370 & 67.952 \\
set 12 & $4.174 \mathrm{e}-04$ & $3.896 \mathrm{e}-01$ & 15.513 & 109.408 & 79.920 \\
set 13 & $3.053 \mathrm{e}-04$ & $9.968 \mathrm{e}-02$ & 146.212 & 169.491 & 37.517 \\
set 14 & $6.325 \mathrm{e}-05$ & $2.725 \mathrm{e}-01$ & 111.862 & 18.260 & 95.724 \\
set 15 & $7.509 \mathrm{e}-04$ & $3.716 \mathrm{e}-02$ & 89.311 & 34.377 & 68.987 \\
set 16 & $3.894 \mathrm{e}-04$ & $4.392 \mathrm{e}-01$ & 139.903 & 104.546 & 175.895 \\
set 17 & $6.266 \mathrm{e}-04$ & $5.300 \mathrm{e}-01$ & 97.707 & 134.203 & 75.327 \\
set 18 & $5.734 \mathrm{e}-04$ & $4.141 \mathrm{e}-01$ & 65.209 & 59.596 & 26.902 \\
set 19 & $8.262 \mathrm{e}-04$ & $1.166 \mathrm{e}-01$ & 72.375 & 105.585 & 16.560 \\
set 20 & $6.232 \mathrm{e}-04$ & $6.364 \mathrm{e}-01$ & 125.130 & 25.316 & 81.669
\end{tabular}

Note that the Euler angles differ due to the different reference frames. Thus a comparison can here only be based on $A_{\mathrm{ZZ}}$ and $R$, which shows a good agreement for the runs 1-A17 to 1-A39 between the input orientations used to back-calculate the artificial RDCs and the final orientations determined by TITANIA. The comparison of orientations with using a common reference frame is found in section 2.2.4. 


\section{1-B}

\section{Input orientations}

The RDCs of setup 1-B were calculated by the orientations summarized in table S-8.

Table S-8: Orientational data used for the synthetic RDC sets of setup 1-B and 1-E.

\begin{tabular}{l|rcrrr} 
Set & \multicolumn{1}{|c}{$\boldsymbol{A}_{\mathrm{zz}}$} & \multicolumn{1}{c}{$\boldsymbol{R}$} & \multicolumn{1}{c}{$\boldsymbol{\alpha} /{ }^{\circ}$} & \multicolumn{1}{c}{$\boldsymbol{\beta} /{ }^{\circ}$} & \multicolumn{1}{c}{$\boldsymbol{\gamma} /^{\circ}$} \\
\hline set 1 & $-8.166 \mathrm{e}-04$ & $4.731 \mathrm{e}-01$ & 62.238 & 154.576 & 121.551 \\
set 2 & $9.519 \mathrm{e}-04$ & $4.642 \mathrm{e}-01$ & 76.734 & 78.357 & 140.473 \\
set 3 & $-1.583 \mathrm{e}-03$ & $3.447 \mathrm{e}-01$ & 148.367 & 118.389 & 150.530 \\
set 4 & $6.259 \mathrm{e}-04$ & $6.557 \mathrm{e}-01$ & 165.587 & 54.317 & 77.300 \\
set 5 & $6.454 \mathrm{e}-04$ & $5.588 \mathrm{e}-01$ & 164.877 & 71.897 & 73.177 \\
set 6 & $-8.756 \mathrm{e}-04$ & $4.057 \mathrm{e}-01$ & 42.558 & 122.051 & 136.107
\end{tabular}

\section{Output orientations}

All orientiations of setup 1-B (11-39) obtained from the full TITANIA optimization runs are listed in the following section. Changes of the Euler angles compared to the literature data listed above are due to the change of the reference frames (TITANIA used the principle axis system of the molecule). The comparison of the input and output data is summarized in table S-36 (section 2.2.4).

Table S-9: Orientational data of IPC 1 obtained by TITANIA on the last optimization iteration for the run 1-B11.

\begin{tabular}{l|rrrrr} 
Set & \multicolumn{1}{|c}{$\boldsymbol{A}_{\mathrm{zz}}$} & \multicolumn{1}{c}{$\boldsymbol{\alpha}$} & \multicolumn{1}{c}{$/^{\circ}$} & \multicolumn{1}{c}{$\boldsymbol{\beta} /{ }^{\circ}$} & \multicolumn{1}{c}{$\boldsymbol{\gamma} /^{\circ}$} \\
\hline set 1 & $-8.244 \mathrm{e}-04$ & $5.073 \mathrm{e}-01$ & 99.505 & 37.190 & 147.052 \\
set 2 & $1.002 \mathrm{e}-03$ & $4.400 \mathrm{e}-01$ & 95.072 & 115.134 & 162.170 \\
set 3 & $-1.619 \mathrm{e}-03$ & $3.989 \mathrm{e}-01$ & 168.306 & 83.003 & 140.187 \\
set 4 & $-7.062 \mathrm{e}-04$ & $5.614 \mathrm{e}-01$ & 160.472 & 99.745 & 137.362 \\
set 5 & $7.119 \mathrm{e}-04$ & $5.892 \mathrm{e}-01$ & 174.633 & 87.953 & 47.321 \\
set 6 & $-7.918 \mathrm{e}-04$ & $2.987 \mathrm{e}-01$ & 61.637 & 72.685 & 153.102
\end{tabular}


Table S-10: Orientational data of IPC 1 obtained by TITANIA on the last optimization iteration of run 1-B17.

\begin{tabular}{l|rrrrr} 
Set & \multicolumn{1}{|c}{$\boldsymbol{A}_{\mathrm{zz}}$} & \multicolumn{1}{c}{$\boldsymbol{R}$} & \multicolumn{1}{c}{$\boldsymbol{\alpha} /{ }^{\circ}$} & \multicolumn{1}{c}{$\boldsymbol{\beta} /{ }^{\circ}$} & \multicolumn{1}{c}{$\boldsymbol{\gamma} /^{\circ}$} \\
\hline set 1 & $-8.108 \mathrm{e}-04$ & $4.409 \mathrm{e}-01$ & 99.643 & 34.298 & 144.649 \\
set 2 & $9.064 \mathrm{e}-04$ & $5.071 \mathrm{e}-01$ & 95.256 & 110.676 & 163.366 \\
set 3 & $-1.505 \mathrm{e}-03$ & $3.608 \mathrm{e}-01$ & 163.909 & 74.951 & 141.586 \\
set 4 & $6.048 \mathrm{e}-04$ & $6.488 \mathrm{e}-01$ & 176.158 & 67.332 & 49.982 \\
set 5 & $6.081 \mathrm{e}-04$ & $5.628 \mathrm{e}-01$ & 178.879 & 84.302 & 48.337 \\
set 6 & $-8.311 \mathrm{e}-04$ & $3.977 \mathrm{e}-01$ & 63.102 & 66.832 & 151.953
\end{tabular}

Table S-11: Orientational data of IPC 1 obtained by TITANIA on the last optimization iteration of run 1-B23.

\begin{tabular}{l|rrrrr} 
Set & \multicolumn{1}{|c}{$\boldsymbol{A}_{\mathrm{zz}}$} & \multicolumn{1}{c}{$\boldsymbol{R}$} & \multicolumn{1}{c}{$\boldsymbol{\alpha} /{ }^{\circ}$} & \multicolumn{1}{c}{$\boldsymbol{\beta} /{ }^{\circ}$} & \multicolumn{1}{c}{$\boldsymbol{\gamma} /^{\circ}$} \\
\hline set 1 & $-8.042 \mathrm{e}-04$ & $4.571 \mathrm{e}-01$ & 96.528 & 31.560 & 149.039 \\
set 2 & $9.047 \mathrm{e}-04$ & $4.945 \mathrm{e}-01$ & 92.854 & 108.102 & 164.157 \\
set 3 & $-1.527 \mathrm{e}-03$ & $3.440 \mathrm{e}-01$ & 161.588 & 71.675 & 144.470 \\
set 4 & $6.048 \mathrm{e}-04$ & $6.509 \mathrm{e}-01$ & 179.884 & 65.253 & 50.975 \\
set 5 & $6.104 \mathrm{e}-04$ & $5.683 \mathrm{e}-01$ & 2.499 & 83.064 & 50.439 \\
set 6 & $-8.505 \mathrm{e}-04$ & $3.968 \mathrm{e}-01$ & 62.457 & 63.994 & 154.884
\end{tabular}

Table S-12: Orientational data of IPC 1 obtained by TITANIA on the last optimization iteration of run 1-B31.

\begin{tabular}{l|rrrrr} 
Set & \multicolumn{1}{|c}{$\boldsymbol{A}_{\mathrm{zz}}$} & \multicolumn{1}{c}{$\boldsymbol{R}$} & \multicolumn{1}{c}{$\boldsymbol{\alpha} /{ }^{\circ}$} & \multicolumn{1}{c}{$\boldsymbol{\beta} /{ }^{\circ}$} & \multicolumn{1}{c}{$\boldsymbol{\gamma}{ }^{\circ}$} \\
\hline set 1 & $-7.644 \mathrm{e}-04$ & $4.726 \mathrm{e}-01$ & 98.228 & 31.031 & 149.711 \\
set 2 & $8.819 \mathrm{e}-04$ & $4.809 \mathrm{e}-01$ & 92.649 & 107.750 & 165.933 \\
set 3 & $-1.468 \mathrm{e}-03$ & $3.562 \mathrm{e}-01$ & 163.680 & 71.780 & 144.397 \\
set 4 & $5.857 \mathrm{e}-04$ & $6.520 \mathrm{e}-01$ & 179.713 & 66.985 & 51.253 \\
set 5 & $6.025 \mathrm{e}-04$ & $5.575 \mathrm{e}-01$ & 2.748 & 84.854 & 50.808 \\
set 6 & $-7.994 \mathrm{e}-04$ & $4.049 \mathrm{e}-01$ & 62.748 & 64.052 & 155.672
\end{tabular}


Table S-13: Orientational data of IPC 1 obtained by TITANIA on the last optimization iteration of run 1-B39.

\begin{tabular}{l|rcrrr} 
Set & \multicolumn{1}{|c}{$\boldsymbol{A}_{\mathrm{zz}}$} & \multicolumn{1}{c}{$\boldsymbol{R}$} & \multicolumn{1}{c}{$\boldsymbol{\alpha} /{ }^{\circ}$} & \multicolumn{1}{c}{$\boldsymbol{\beta} /{ }^{\circ}$} & \multicolumn{1}{c}{$\boldsymbol{\gamma} /^{\circ}$} \\
\hline set 1 & $-7.947 \mathrm{e}-04$ & $4.634 \mathrm{e}-01$ & 97.953 & 34.319 & 144.673 \\
set 2 & $9.067 \mathrm{e}-04$ & $4.808 \mathrm{e}-01$ & 95.439 & 110.469 & 162.358 \\
set 3 & $-1.522 \mathrm{e}-03$ & $3.504 \mathrm{e}-01$ & 163.308 & 74.337 & 141.465 \\
set 4 & $6.066 \mathrm{e}-04$ & $6.455 \mathrm{e}-01$ & 176.995 & 66.514 & 49.234 \\
set 5 & $6.213 \mathrm{e}-04$ & $5.514 \mathrm{e}-01$ & 0.137 & 84.036 & 47.788 \\
set 6 & $-8.415 \mathrm{e}-04$ & $4.056 \mathrm{e}-01$ & 62.515 & 66.982 & 152.650
\end{tabular}

Note that as before the Euler angles differ due to the different reference frames. Thus a comparison can here only be based on $A_{\mathrm{ZZ}}$ and $R$, which again show a good agreement between the input orientations used for backcalculation and the final orientations for the runs utilizing 17 to 39 RDCs. The comparison of orientations with using a common reference frame is found in section 2.2.4. 


\section{1-C}

\section{Input orientations}

The RDCs of setup 1-C were calculated by the orientations summarized in table S-14.

Table S-14: Orientational used for the synthetic RDC sets of setup 1-C and 1-F.

\begin{tabular}{l|rcrrr} 
Set & \multicolumn{1}{|c}{$\boldsymbol{A}_{\mathrm{zz}}$} & \multicolumn{1}{c}{$\boldsymbol{R}$} & $\boldsymbol{\alpha} /{ }^{\circ}$ & \multicolumn{1}{c}{$\boldsymbol{\beta} /{ }^{\circ}$} & \multicolumn{1}{c}{$\boldsymbol{\gamma}{ }^{\circ}$} \\
\hline set 1 & $-8.166 \mathrm{e}-04$ & $4.731 \mathrm{e}-01$ & 62.238 & 154.576 & 121.551 \\
set 2 & $9.519 \mathrm{e}-04$ & $4.642 \mathrm{e}-01$ & 76.734 & 78.357 & 140.473 \\
set 7 & $-6.930 \mathrm{e}-04$ & $6.207 \mathrm{e}-01$ & 67.088 & 140.025 & 118.143 \\
set 8 & $9.806 \mathrm{e}-04$ & $5.071 \mathrm{e}-01$ & 77.192 & 81.428 & 137.636 \\
set 9 & $1.036 \mathrm{e}-03$ & $5.134 \mathrm{e}-01$ & 72.293 & 80.309 & 139.775 \\
set 10 & $9.246 \mathrm{e}-04$ & $4.815 \mathrm{e}-01$ & 77.352 & 77.964 & 140.567
\end{tabular}

\section{Output orientations}

All orientations of setup 1-C (11-39) obtained from the full TITANIA optimization runs are listed in the following section. Changes of the Euler angles compared to the literature data listed above are due to the change of the reference frames (TITANIA used the principle axis system of the molecule). The comparison of the input and output data is summarized in table S-37 (section 2.2.4).

Table S-15: Orientational data of IPC 1 obtained by TITANIA on the last optimization iteration for the runs 1-C11.

\begin{tabular}{l|rcrrr} 
Set & \multicolumn{1}{|c}{$\boldsymbol{A}_{\mathrm{zz}}$} & \multicolumn{1}{c}{$\boldsymbol{R}$} & \multicolumn{1}{c}{$\boldsymbol{\alpha} /{ }^{\circ}$} & \multicolumn{1}{c}{$\boldsymbol{\beta} /{ }^{\circ}$} & \multicolumn{1}{c}{$\boldsymbol{\gamma}{ }^{\circ}$} \\
\hline set 1 & $-7.943 \mathrm{e}-04$ & $5.097 \mathrm{e}-01$ & 102.085 & 37.179 & 143.291 \\
set 2 & $9.609 \mathrm{e}-04$ & $4.326 \mathrm{e}-01$ & 100.119 & 113.990 & 162.818 \\
set 7 & $-6.135 \mathrm{e}-04$ & $6.637 \mathrm{e}-01$ & 98.069 & 48.535 & 160.754 \\
set 8 & $9.945 \mathrm{e}-04$ & $4.811 \mathrm{e}-01$ & 101.804 & 110.641 & 164.537 \\
set 9 & $1.050 \mathrm{e}-03$ & $4.726 \mathrm{e}-01$ & 96.439 & 112.234 & 163.051 \\
set 10 & $9.343 \mathrm{e}-04$ & $4.500 \mathrm{e}-01$ & 100.587 & 114.356 & 162.903
\end{tabular}


Table S-16: Orientational data of IPC 1 obtained by TITANIA on the last optimization iteration of run 1-C17.

\begin{tabular}{l|rcrrr} 
Set & \multicolumn{1}{|c}{$\boldsymbol{A}_{\mathrm{zz}}$} & $\boldsymbol{R}$ & $\boldsymbol{\alpha} /{ }^{\circ}$ & \multicolumn{1}{c}{$\boldsymbol{\beta} /{ }^{\circ}$} & \multicolumn{1}{c}{$\boldsymbol{\gamma} /^{\circ}$} \\
\hline set 1 & $-6.630 \mathrm{e}-04$ & $5.320 \mathrm{e}-01$ & 92.012 & 27.294 & 149.210 \\
set 2 & $6.131 \mathrm{e}-04$ & $4.682 \mathrm{e}-01$ & 81.440 & 102.747 & 153.765 \\
set 7 & $6.536 \mathrm{e}-04$ & $6.242 \mathrm{e}-01$ & 92.776 & 128.778 & 165.380 \\
set 8 & $5.919 \mathrm{e}-04$ & $5.567 \mathrm{e}-01$ & 82.873 & 96.917 & 153.684 \\
set 9 & $6.396 \mathrm{e}-04$ & $5.731 \mathrm{e}-01$ & 74.931 & 98.582 & 151.072 \\
set 10 & $5.998 \mathrm{e}-04$ & $4.847 \mathrm{e}-01$ & 82.711 & 103.363 & 153.929
\end{tabular}

Table S-17: Orientational data of IPC 1 obtained by TITANIA on the last optimization iteration of run 1-C23.

\begin{tabular}{l|rrrrr} 
Set & \multicolumn{1}{|c}{$\boldsymbol{A}_{\mathrm{zz}}$} & \multicolumn{1}{c}{$\boldsymbol{\alpha}$} & $\boldsymbol{\alpha} /{ }^{\circ}$ & \multicolumn{1}{c}{$\boldsymbol{\beta} /{ }^{\circ}$} & \multicolumn{1}{c}{$\boldsymbol{\gamma}{ }^{\circ}$} \\
\hline set 1 & $-8.047 \mathrm{e}-04$ & $4.573 \mathrm{e}-01$ & 96.356 & 31.573 & 149.123 \\
set 2 & $9.060 \mathrm{e}-04$ & $4.933 \mathrm{e}-01$ & 92.842 & 108.112 & 164.115 \\
set 7 & $-6.871 \mathrm{e}-04$ & $5.963 \mathrm{e}-01$ & 93.761 & 43.221 & 162.469 \\
set 8 & $9.305 \mathrm{e}-04$ & $5.356 \mathrm{e}-01$ & 93.201 & 104.329 & 165.855 \\
set 9 & $9.846 \mathrm{e}-04$ & $5.441 \mathrm{e}-01$ & 88.538 & 105.973 & 164.218 \\
set 10 & $8.806 \mathrm{e}-04$ & $5.106 \mathrm{e}-01$ & 93.458 & 108.524 & 164.143
\end{tabular}

Table S-18: Orientational data of IPC 1 obtained by TITANIA on the last optimization iteration of run 1-C31.

\begin{tabular}{l|rcrrr} 
Set & \multicolumn{1}{|c}{$\boldsymbol{A}_{\text {zz }}$} & \multicolumn{1}{c}{} & $\boldsymbol{\alpha} /{ }^{\circ}$ & \multicolumn{1}{c}{$\boldsymbol{\beta} /{ }^{\circ}$} & \multicolumn{1}{c}{$\boldsymbol{\gamma}{ }^{\circ}$} \\
\hline set 1 & $-7.950 \mathrm{e}-04$ & $4.621 \mathrm{e}-01$ & 97.104 & 31.388 & 149.865 \\
set 2 & $9.152 \mathrm{e}-04$ & $4.760 \mathrm{e}-01$ & 93.208 & 108.092 & 165.652 \\
set 7 & $-6.720 \mathrm{e}-04$ & $6.171 \mathrm{e}-01$ & 93.379 & 42.998 & 164.303 \\
set 8 & $9.408 \mathrm{e}-04$ & $5.198 \mathrm{e}-01$ & 93.779 & 104.373 & 167.514 \\
set 9 & $9.951 \mathrm{e}-04$ & $5.254 \mathrm{e}-01$ & 88.804 & 106.031 & 165.771 \\
set 10 & $8.891 \mathrm{e}-04$ & $4.935 \mathrm{e}-01$ & 93.852 & 108.487 & 165.691
\end{tabular}


Table S-19: Orientational data of IPC 1 obtained by TITANIA on the last optimization iteration of run 1-C39.

\begin{tabular}{l|rcrrr} 
Set & \multicolumn{1}{|c}{$\boldsymbol{A}_{\mathrm{zz}}$} & \multicolumn{1}{c}{$\boldsymbol{\alpha}$} & $\boldsymbol{\alpha} /{ }^{\circ}$ & \multicolumn{1}{c}{$\boldsymbol{\beta} /{ }^{\circ}$} & \multicolumn{1}{c}{$\boldsymbol{\gamma} /^{\circ}$} \\
\hline set 1 & $-7.839 \mathrm{e}-04$ & $4.699 \mathrm{e}-01$ & 96.697 & 31.199 & 149.514 \\
set 2 & $8.978 \mathrm{e}-04$ & $4.811 \mathrm{e}-01$ & 93.376 & 107.944 & 165.281 \\
set 7 & $-6.608 \mathrm{e}-04$ & $6.242 \mathrm{e}-01$ & 92.838 & 42.771 & 164.059 \\
set 8 & $9.221 \mathrm{e}-04$ & $5.260 \mathrm{e}-01$ & 93.844 & 104.220 & 167.183 \\
set 9 & $9.754 \mathrm{e}-04$ & $5.309 \mathrm{e}-01$ & 88.906 & 105.870 & 165.412 \\
set 10 & $8.726 \mathrm{e}-04$ & $4.984 \mathrm{e}-01$ & 93.995 & 108.343 & 165.304
\end{tabular}

Note that as before the Euler angles differ due to the different reference frames. Thus a comparison can here only be based on $A_{\mathrm{ZZ}}$ and $R$, which again show a good agreement between the input orientations used for backcalculation and the final orientations for the runs utilizing 17 to 39 RDCs. The comparison of orientations with using a common reference frame is found in section 2.2.4. 


\section{$1-\mathrm{D}$}

\section{Input orientations}

The RDCs of run 1-D were calculated by adding random Gaussian error to the RDCs of 1-A (for more information see the start of section 2). Therefore the orientation data are reported in table S-2.

\section{Output orientations}

All orientiations of run 1-D (11-39) obtained from the full TITANIA optimization runs are listed in the following section. Changes of the Euler angles compared to the literature data listed above are due to the change of the reference frames (TITANIA used the principle axis system of the molecule) and the added errors. The comparison of the input and output data is summarized in table S-38 (section 2.2.4).

Table S-20: Orientational data of IPC 1 obtained by TITANIA on the last optimization iteration for the runs 1-D11.

\begin{tabular}{l|rcrrr} 
Set & \multicolumn{1}{|c}{$\boldsymbol{A}_{\text {zz }}$} & \multicolumn{1}{c}{$\boldsymbol{\alpha} /{ }^{\circ}$} & \multicolumn{1}{c}{$\boldsymbol{\beta} /{ }^{\circ}$} & \multicolumn{1}{c}{$\boldsymbol{\gamma} /{ }^{\circ}$} \\
\hline set 1 & $6.563 \mathrm{e}-04$ & $1.709 \mathrm{e}-01$ & 17.261 & 34.845 & 91.053 \\
set 2 & $-5.517 \mathrm{e}-05$ & $3.735 \mathrm{e}-01$ & 87.915 & 26.830 & 9.600 \\
set 3 & $-9.313 \mathrm{e}-05$ & $9.125 \mathrm{e}-02$ & 171.939 & 98.010 & 98.051 \\
set 4 & $4.663 \mathrm{e}-04$ & $5.462 \mathrm{e}-01$ & 93.750 & 121.115 & 64.374 \\
set 5 & $3.359 \mathrm{e}-04$ & $3.032 \mathrm{e}-01$ & 133.863 & 104.485 & 88.760 \\
set 6 & $7.921 \mathrm{e}-04$ & $4.444 \mathrm{e}-01$ & 166.107 & 124.691 & 143.805 \\
set 7 & $7.918 \mathrm{e}-04$ & $1.332 \mathrm{e}-01$ & 147.979 & 49.227 & 54.222 \\
set 8 & $3.386 \mathrm{e}-04$ & $3.093 \mathrm{e}-01$ & 71.237 & 122.130 & 20.822 \\
set 9 & $-6.140 \mathrm{e}-05$ & $2.967 \mathrm{e}-01$ & 105.865 & 34.709 & 133.496 \\
set 10 & $8.866 \mathrm{e}-04$ & $4.802 \mathrm{e}-01$ & 11.296 & 28.311 & 108.368 \\
set 11 & $2.675 \mathrm{e}-04$ & $1.420 \mathrm{e}-01$ & 98.058 & 149.404 & 64.884 \\
set 12 & $4.638 \mathrm{e}-04$ & $1.826 \mathrm{e}-01$ & 10.888 & 114.606 & 78.810 \\
set 13 & $2.887 \mathrm{e}-04$ & $1.143 \mathrm{e}-01$ & 168.817 & 13.701 & 165.191 \\
set 14 & $8.921 \mathrm{e}-05$ & $2.601 \mathrm{e}-01$ & 37.800 & 23.631 & 136.646 \\
set 15 & $8.573 \mathrm{e}-04$ & $1.154 \mathrm{e}-01$ & 92.175 & 34.978 & 73.255 \\
set 16 & $4.080 \mathrm{e}-04$ & $3.538 \mathrm{e}-01$ & 137.808 & 107.276 & 176.617 \\
set 17 & $6.827 \mathrm{e}-04$ & $5.409 \mathrm{e}-01$ & 92.825 & 132.102 & 73.204 \\
set 18 & $5.554 \mathrm{e}-04$ & $2.171 \mathrm{e}-01$ & 49.083 & 55.873 & 29.584 \\
set 19 & $8.126 \mathrm{e}-04$ & $2.133 \mathrm{e}-01$ & 55.817 & 101.724 & 14.943 \\
set 20 & $7.043 \mathrm{e}-04$ & $6.064 \mathrm{e}-01$ & 108.558 & 27.698 & 88.831
\end{tabular}


Table S-21: Orientational data of IPC 1 obtained by TITANIA on the last optimization iteration of run 1-D17.

\begin{tabular}{l|rcrrr} 
Set & \multicolumn{1}{c}{$\boldsymbol{A}_{\mathrm{zz}}$} & $\boldsymbol{R}$ & \multicolumn{1}{c}{$\boldsymbol{\alpha} /{ }^{\circ}$} & \multicolumn{1}{c}{$\boldsymbol{\beta} /{ }^{\circ}$} & \multicolumn{1}{c}{$\boldsymbol{\gamma} /{ }^{\circ}$} \\
\hline set 1 & $6.059 \mathrm{e}-04$ & $3.268 \mathrm{e}-01$ & 13.221 & 33.839 & 89.181 \\
set 2 & $8.460 \mathrm{e}-05$ & $3.694 \mathrm{e}-01$ & 129.989 & 70.139 & 140.494 \\
set 3 & $-1.176 \mathrm{e}-04$ & $6.150 \mathrm{e}-01$ & 164.392 & 86.995 & 117.859 \\
set 4 & $4.040 \mathrm{e}-04$ & $4.402 \mathrm{e}-01$ & 96.585 & 130.964 & 59.756 \\
set 5 & $2.841 \mathrm{e}-04$ & $4.845 \mathrm{e}-01$ & 156.371 & 104.724 & 98.880 \\
set 6 & $8.591 \mathrm{e}-04$ & $4.121 \mathrm{e}-01$ & 165.522 & 129.724 & 150.550 \\
set 7 & $8.145 \mathrm{e}-04$ & $6.008 \mathrm{e}-02$ & 11.020 & 56.983 & 56.894 \\
set 8 & $4.182 \mathrm{e}-04$ & $1.440 \mathrm{e}-01$ & 81.533 & 126.561 & 20.323 \\
set 9 & $7.065 \mathrm{e}-05$ & $2.541 \mathrm{e}-01$ & 123.750 & 146.150 & 161.212 \\
set 10 & $7.675 \mathrm{e}-04$ & $5.517 \mathrm{e}-01$ & 15.325 & 21.610 & 99.638 \\
set 11 & $3.710 \mathrm{e}-04$ & $2.226 \mathrm{e}-01$ & 43.364 & 158.730 & 65.707 \\
set 12 & $4.143 \mathrm{e}-04$ & $4.879 \mathrm{e}-01$ & 14.950 & 119.941 & 83.078 \\
set 13 & $3.195 \mathrm{e}-04$ & $1.952 \mathrm{e}-01$ & 166.385 & 168.871 & 10.701 \\
set 14 & $7.742 \mathrm{e}-05$ & $4.860 \mathrm{e}-01$ & 111.691 & 10.102 & 93.346 \\
set 15 & $7.571 \mathrm{e}-04$ & $5.801 \mathrm{e}-02$ & 178.908 & 40.032 & 71.258 \\
set 16 & $4.329 \mathrm{e}-04$ & $3.027 \mathrm{e}-01$ & 33.782 & 72.276 & 2.236 \\
set 17 & $6.593 \mathrm{e}-04$ & $4.945 \mathrm{e}-01$ & 93.732 & 142.102 & 72.712 \\
set 18 & $6.516 \mathrm{e}-04$ & $3.550 \mathrm{e}-01$ & 50.779 & 61.785 & 35.678 \\
set 19 & $8.827 \mathrm{e}-04$ & $8.288 \mathrm{e}-02$ & 77.402 & 103.840 & 16.179 \\
set 20 & $-6.316 \mathrm{e}-04$ & $5.429 \mathrm{e}-01$ & 110.204 & 115.878 & 122.231
\end{tabular}


Table S-22: Orientational data of IPC 1 obtained by TITANIA on the last optimization iteration of run 1-D23.

\begin{tabular}{l|rcrrr} 
Set & \multicolumn{1}{c}{$\boldsymbol{A}_{\mathrm{zz}}$} & $\boldsymbol{R}$ & \multicolumn{1}{c}{$\boldsymbol{\alpha} /{ }^{\circ}$} & \multicolumn{1}{c}{$\boldsymbol{\beta} /{ }^{\circ}$} & \multicolumn{1}{c}{$\boldsymbol{\gamma} /{ }^{\circ}$} \\
\hline set 1 & $5.426 \mathrm{e}-04$ & $2.362 \mathrm{e}-01$ & 11.316 & 32.424 & 88.929 \\
set 2 & $7.098 \mathrm{e}-05$ & $2.009 \mathrm{e}-01$ & 109.314 & 70.870 & 152.829 \\
set 3 & $-9.066 \mathrm{e}-05$ & $5.977 \mathrm{e}-01$ & 159.380 & 70.140 & 116.061 \\
set 4 & $4.470 \mathrm{e}-04$ & $3.137 \mathrm{e}-01$ & 97.587 & 127.566 & 57.891 \\
set 5 & $2.761 \mathrm{e}-04$ & $3.854 \mathrm{e}-01$ & 140.338 & 105.367 & 88.578 \\
set 6 & $7.891 \mathrm{e}-04$ & $4.065 \mathrm{e}-01$ & 172.713 & 124.737 & 147.775 \\
set 7 & $7.637 \mathrm{e}-04$ & $3.626 \mathrm{e}-02$ & 178.086 & 53.274 & 50.632 \\
set 8 & $4.147 \mathrm{e}-04$ & $1.731 \mathrm{e}-01$ & 85.358 & 128.168 & 25.998 \\
set 9 & $-6.384 \mathrm{e}-05$ & $5.674 \mathrm{e}-01$ & 114.262 & 46.157 & 128.209 \\
set 10 & $7.141 \mathrm{e}-04$ & $5.443 \mathrm{e}-01$ & 9.243 & 22.310 & 105.819 \\
set 11 & $3.569 \mathrm{e}-04$ & $1.704 \mathrm{e}-01$ & 36.869 & 156.062 & 61.174 \\
set 12 & $4.286 \mathrm{e}-04$ & $4.063 \mathrm{e}-01$ & 15.881 & 115.892 & 75.844 \\
set 13 & $3.301 \mathrm{e}-04$ & $9.256 \mathrm{e}-02$ & 160.233 & 167.061 & 13.655 \\
set 14 & $8.555 \mathrm{e}-05$ & $4.025 \mathrm{e}-01$ & 73.338 & 6.196 & 134.808 \\
set 15 & $7.036 \mathrm{e}-04$ & $5.993 \mathrm{e}-02$ & 120.607 & 36.416 & 67.786 \\
set 16 & $4.320 \mathrm{e}-04$ & $2.289 \mathrm{e}-01$ & 131.685 & 109.974 & 176.345 \\
set 17 & $6.525 \mathrm{e}-04$ & $4.270 \mathrm{e}-01$ & 90.678 & 138.119 & 67.640 \\
set 18 & $6.318 \mathrm{e}-04$ & $3.737 \mathrm{e}-01$ & 61.248 & 58.735 & 28.034 \\
set 19 & $7.326 \mathrm{e}-04$ & $1.426 \mathrm{e}-01$ & 55.639 & 103.787 & 15.440 \\
set 20 & $-5.794 \mathrm{e}-04$ & $6.602 \mathrm{e}-01$ & 102.764 & 113.157 & 117.951
\end{tabular}


Table S-23: Orientational data of IPC 1 obtained by TITANIA on the last optimization iteration of run 1-D31.

\begin{tabular}{l|rcrrr} 
Set & \multicolumn{1}{c}{$\boldsymbol{A}_{\mathrm{zz}}$} & $\boldsymbol{R}$ & \multicolumn{1}{c}{$\boldsymbol{\alpha} /{ }^{\circ}$} & \multicolumn{1}{c}{$\boldsymbol{\beta} /{ }^{\circ}$} & \multicolumn{1}{c}{$\boldsymbol{\gamma} /{ }^{\circ}$} \\
\hline set 1 & $8.047 \mathrm{e}-04$ & $3.336 \mathrm{e}-01$ & 25.222 & 61.033 & 95.877 \\
set 2 & $-8.554 \mathrm{e}-05$ & $6.285 \mathrm{e}-01$ & 5.810 & 60.847 & 87.329 \\
set 3 & $-1.565 \mathrm{e}-04$ & $5.936 \mathrm{e}-01$ & 3.039 & 51.339 & 115.432 \\
set 4 & $4.875 \mathrm{e}-04$ & $3.627 \mathrm{e}-01$ & 77.128 & 157.071 & 53.823 \\
set 5 & $2.976 \mathrm{e}-04$ & $1.638 \mathrm{e}-01$ & 148.088 & 137.136 & 106.951 \\
set 6 & $1.004 \mathrm{e}-03$ & $4.777 \mathrm{e}-01$ & 155.895 & 59.952 & 0.334 \\
set 7 & $8.694 \mathrm{e}-04$ & $1.285 \mathrm{e}-01$ & 2.828 & 86.872 & 71.023 \\
set 8 & $4.560 \mathrm{e}-04$ & $4.040 \mathrm{e}-02$ & 56.083 & 144.047 & 12.286 \\
set 9 & $8.979 \mathrm{e}-05$ & $5.555 \mathrm{e}-01$ & 71.705 & 21.314 & 96.499 \\
set 10 & $-9.124 \mathrm{e}-04$ & $6.013 \mathrm{e}-01$ & 38.910 & 122.316 & 30.387 \\
set 11 & $3.752 \mathrm{e}-04$ & $1.111 \mathrm{e}-01$ & 72.986 & 10.509 & 106.165 \\
set 12 & $4.872 \mathrm{e}-04$ & $3.780 \mathrm{e}-01$ & 23.796 & 143.958 & 94.570 \\
set 13 & $4.100 \mathrm{e}-04$ & $6.639 \mathrm{e}-02$ & 145.202 & 32.008 & 103.647 \\
set 14 & $-9.756 \mathrm{e}-05$ & $3.743 \mathrm{e}-01$ & 144.083 & 110.067 & 148.322 \\
set 15 & $9.326 \mathrm{e}-04$ & $1.250 \mathrm{e}-01$ & 36.552 & 68.904 & 85.908 \\
set 16 & $4.417 \mathrm{e}-04$ & $4.748 \mathrm{e}-01$ & 176.989 & 90.463 & 10.252 \\
set 17 & $-6.937 \mathrm{e}-04$ & $6.565 \mathrm{e}-01$ & 87.432 & 78.067 & 93.327 \\
set 18 & $6.253 \mathrm{e}-04$ & $6.106 \mathrm{e}-01$ & 46.556 & 89.597 & 46.569 \\
set 19 & $1.034 \mathrm{e}-03$ & $1.708 \mathrm{e}-01$ & 22.402 & 124.759 & 16.561 \\
set 20 & $7.612 \mathrm{e}-04$ & $5.575 \mathrm{e}-01$ & 134.633 & 56.445 & 93.873
\end{tabular}


Table S-24: Orientational data of IPC 1 obtained by TITANIA on the last optimization iteration of run 1-D39.

\begin{tabular}{l|rcrrr} 
Set & \multicolumn{1}{c}{$\boldsymbol{A}_{\text {zz }}$} & \multicolumn{1}{c}{$\boldsymbol{\alpha} /{ }^{\circ}$} & \multicolumn{1}{c}{$\boldsymbol{\beta} /{ }^{\circ}$} & \multicolumn{1}{c}{$\boldsymbol{\gamma} /{ }^{\circ}$} \\
\hline set 1 & $6.137 \mathrm{e}-04$ & $2.661 \mathrm{e}-01$ & 11.846 & 33.966 & 86.087 \\
set 2 & $1.069 \mathrm{e}-04$ & $2.113 \mathrm{e}-01$ & 115.186 & 85.384 & 125.380 \\
set 3 & $-1.376 \mathrm{e}-04$ & $6.411 \mathrm{e}-01$ & 10.793 & 50.272 & 101.518 \\
set 4 & $4.296 \mathrm{e}-04$ & $5.611 \mathrm{e}-01$ & 104.454 & 130.564 & 51.757 \\
set 5 & $2.668 \mathrm{e}-04$ & $4.541 \mathrm{e}-01$ & 135.754 & 111.709 & 81.312 \\
set 6 & $8.533 \mathrm{e}-04$ & $4.253 \mathrm{e}-01$ & 163.643 & 127.678 & 143.549 \\
set 7 & $7.836 \mathrm{e}-04$ & $3.283 \mathrm{e}-02$ & 3.277 & 58.422 & 46.265 \\
set 8 & $4.234 \mathrm{e}-04$ & $2.592 \mathrm{e}-01$ & 106.522 & 131.223 & 15.142 \\
set 9 & $-9.219 \mathrm{e}-05$ & $3.046 \mathrm{e}-01$ & 90.650 & 69.495 & 30.447 \\
set 10 & $6.654 \mathrm{e}-04$ & $5.674 \mathrm{e}-01$ & 26.150 & 22.286 & 86.476 \\
set 11 & $3.389 \mathrm{e}-04$ & $9.875 \mathrm{e}-02$ & 80.190 & 157.003 & 48.210 \\
set 12 & $4.771 \mathrm{e}-04$ & $1.638 \mathrm{e}-01$ & 172.144 & 120.901 & 75.174 \\
set 13 & $3.494 \mathrm{e}-04$ & $2.126 \mathrm{e}-01$ & 89.884 & 3.648 & 111.646 \\
set 14 & $8.111 \mathrm{e}-05$ & $4.186 \mathrm{e}-01$ & 155.709 & 15.863 & 62.243 \\
set 15 & $7.576 \mathrm{e}-04$ & $1.021 \mathrm{e}-01$ & 127.889 & 40.833 & 62.219 \\
set 16 & $3.193 \mathrm{e}-04$ & $4.718 \mathrm{e}-01$ & 149.408 & 97.659 & 168.665 \\
set 17 & $6.339 \mathrm{e}-04$ & $5.178 \mathrm{e}-01$ & 106.807 & 138.658 & 76.344 \\
set 18 & $5.853 \mathrm{e}-04$ & $4.836 \mathrm{e}-01$ & 56.279 & 68.670 & 23.333 \\
set 19 & $8.351 \mathrm{e}-04$ & $1.388 \mathrm{e}-01$ & 82.687 & 111.111 & 9.466 \\
set 20 & $-6.623 \mathrm{e}-04$ & $6.587 \mathrm{e}-01$ & 111.998 & 113.253 & 112.885
\end{tabular}

In contrast to the setups $\mathbf{1 - A}$ to $\mathbf{1 - C}$ the Euler angles not only differ due to the different reference frames but also due to the heterogeneity added purposefully. This heterogeneity will also have an impact on $A_{\mathrm{ZZ}}$ and $R$, which is why larger differences between the input orientations used for backcalculation and the final orientations are found. These differences show variations between the respective sets of one run. The reason for this finding is the different magnitudes of $A_{\mathrm{ZZ}}$ used for the back-calculation of the data (see table S-2). This circumstance is discussed below in more detail (see table S-41). The comparison of the orientations using a common reference frame as discussed before is found in section 2.2.4. 


\section{1-E}

\section{Input orientations}

The RDCs of run 1-E were calculated by adding random Gaussian error to the RDCs of 1-B (for more information see the start of section 2). Therefore the orientation data are reported in table S-8.

\section{Output orientations}

All orientiations of setup 1-E (11-39) obtained from the full TITANIA optimization runs are listed in the following section. Changes of the Euler angles compared to the literature data listed above are due to the change of the reference frames (TITANIA used the principle axis system of the molecule) and the added errors. The comparison of the input and output data is summarized in table S-39 (section 2.2.4).

Table S-25: Orientational data of IPC 1 obtained by TITANIA on the last optimization iteration for the runs 1-E11.

\begin{tabular}{l|rcrrr} 
Set & \multicolumn{1}{|c}{$\boldsymbol{A}_{\mathrm{zz}}$} & \multicolumn{1}{c}{$\boldsymbol{\alpha}$} & \multicolumn{1}{c}{$/^{\circ}$} & \multicolumn{1}{c}{$\boldsymbol{\beta} /{ }^{\circ}$} & \multicolumn{1}{c}{$\boldsymbol{\gamma} /{ }^{\circ}$} \\
\hline set 1 & $-7.950 \mathrm{e}-04$ & $4.150 \mathrm{e}-01$ & 98.308 & 39.844 & 148.548 \\
set 2 & $1.075 \mathrm{e}-03$ & $3.454 \mathrm{e}-01$ & 95.275 & 110.848 & 170.903 \\
set 3 & $-1.715 \mathrm{e}-03$ & $2.654 \mathrm{e}-01$ & 166.563 & 81.713 & 150.363 \\
set 4 & $6.906 \mathrm{e}-04$ & $5.243 \mathrm{e}-01$ & 174.590 & 61.131 & 62.663 \\
set 5 & $-6.504 \mathrm{e}-04$ & $6.572 \mathrm{e}-01$ & 171.204 & 92.325 & 148.200 \\
set 6 & $-1.112 \mathrm{e}-03$ & $4.359 \mathrm{e}-01$ & 57.118 & 78.351 & 160.360
\end{tabular}

Table S-26: Orientational data of IPC 1 obtained by TITANIA on the last optimization iteration of run 1-E17.

\begin{tabular}{l|rrrrr} 
Set & \multicolumn{1}{|c}{$\boldsymbol{A}_{\mathrm{zz}}$} & \multicolumn{1}{c}{$\boldsymbol{R}$} & \multicolumn{1}{c}{$\boldsymbol{\alpha} /{ }^{\circ}$} & \multicolumn{1}{c}{$\boldsymbol{\beta} /{ }^{\circ}$} & \multicolumn{1}{c}{$\boldsymbol{\gamma} /^{\circ}$} \\
\hline set 1 & $-8.416 \mathrm{e}-04$ & $4.807 \mathrm{e}-01$ & 104.721 & 32.283 & 145.280 \\
set 2 & $1.028 \mathrm{e}-03$ & $4.582 \mathrm{e}-01$ & 93.978 & 109.339 & 167.592 \\
set 3 & $-1.505 \mathrm{e}-03$ & $3.815 \mathrm{e}-01$ & 171.934 & 74.350 & 138.887 \\
set 4 & $6.274 \mathrm{e}-04$ & $5.836 \mathrm{e}-01$ & 177.183 & 69.383 & 50.276 \\
set 5 & $6.333 \mathrm{e}-04$ & $5.853 \mathrm{e}-01$ & 1.499 & 89.880 & 48.709 \\
set 6 & $-8.158 \mathrm{e}-04$ & $3.788 \mathrm{e}-01$ & 64.006 & 66.422 & 148.850
\end{tabular}


Table S-27: Orientational data of IPC 1 obtained by TITANIA on the last optimization iteration of run 1-E23.

\begin{tabular}{l|rrrrr} 
Set & \multicolumn{1}{|c}{$\boldsymbol{A}_{\text {zz }}$} & \multicolumn{1}{c}{$\boldsymbol{\alpha}$} & \multicolumn{1}{c}{$/^{\circ}$} & \multicolumn{1}{c}{$\boldsymbol{\beta} /{ }^{\circ}$} & \multicolumn{1}{c}{$\boldsymbol{\gamma} /^{\circ}$} \\
\hline set 1 & $-7.845 \mathrm{e}-04$ & $5.005 \mathrm{e}-01$ & 111.550 & 33.680 & 133.099 \\
set 2 & $9.726 \mathrm{e}-04$ & $4.230 \mathrm{e}-01$ & 103.895 & 107.315 & 165.964 \\
set 3 & $-1.378 \mathrm{e}-03$ & $3.799 \mathrm{e}-01$ & 167.469 & 76.387 & 142.910 \\
set 4 & $6.171 \mathrm{e}-04$ & $5.638 \mathrm{e}-01$ & 174.011 & 63.799 & 53.724 \\
set 5 & $5.885 \mathrm{e}-04$ & $5.784 \mathrm{e}-01$ & 176.935 & 83.091 & 50.360 \\
set 6 & $-8.206 \mathrm{e}-04$ & $3.945 \mathrm{e}-01$ & 62.038 & 68.148 & 152.355
\end{tabular}

Table S-28: Orientational data of IPC 1 obtained by TITANIA on the last optimization iteration of run 1-E31.

\begin{tabular}{l|rrrrr} 
Set & \multicolumn{1}{|c}{$\boldsymbol{A}_{\mathrm{zz}}$} & \multicolumn{1}{c}{$\boldsymbol{R}$} & \multicolumn{1}{c}{$\boldsymbol{\alpha} /{ }^{\circ}$} & \multicolumn{1}{c}{$\boldsymbol{\beta} /{ }^{\circ}$} & \multicolumn{1}{c}{$\boldsymbol{\gamma} /^{\circ}$} \\
\hline set 1 & $-7.148 \mathrm{e}-04$ & $4.847 \mathrm{e}-01$ & 111.265 & 34.675 & 139.686 \\
set 2 & $8.980 \mathrm{e}-04$ & $4.268 \mathrm{e}-01$ & 96.474 & 108.629 & 170.759 \\
set 3 & $-1.481 \mathrm{e}-03$ & $3.565 \mathrm{e}-01$ & 174.799 & 79.481 & 140.420 \\
set 4 & $-5.675 \mathrm{e}-04$ & $6.589 \mathrm{e}-01$ & 165.145 & 96.850 & 141.009 \\
set 5 & $5.897 \mathrm{e}-04$ & $6.332 \mathrm{e}-01$ & 174.093 & 95.134 & 48.894 \\
set 6 & $-8.324 \mathrm{e}-04$ & $3.884 \mathrm{e}-01$ & 71.951 & 70.827 & 148.756
\end{tabular}

Table S-29: Orientational data of IPC 1 obtained by TITANIA on the last optimization iteration of run 1-E39.

\begin{tabular}{l|rrrrr} 
Set & \multicolumn{1}{|c}{$\boldsymbol{A}_{\mathrm{zz}}$} & \multicolumn{1}{c}{$\boldsymbol{R}$} & \multicolumn{1}{c}{$\boldsymbol{\alpha} /{ }^{\circ}$} & \multicolumn{1}{c}{$\boldsymbol{\beta} /{ }^{\circ}$} & \multicolumn{1}{c}{$\boldsymbol{\gamma}{ }^{\circ}$} \\
\hline set 1 & $-7.372 \mathrm{e}-04$ & $4.911 \mathrm{e}-01$ & 101.629 & 34.887 & 153.570 \\
set 2 & $9.537 \mathrm{e}-04$ & $4.505 \mathrm{e}-01$ & 91.551 & 112.601 & 176.219 \\
set 3 & $-1.408 \mathrm{e}-03$ & $4.446 \mathrm{e}-01$ & 174.426 & 81.373 & 150.820 \\
set 4 & $-6.019 \mathrm{e}-04$ & $6.482 \mathrm{e}-01$ & 164.776 & 98.050 & 146.706 \\
set 5 & $6.340 \mathrm{e}-04$ & $6.218 \mathrm{e}-01$ & 172.237 & 91.176 & 55.083 \\
set 6 & $-8.176 \mathrm{e}-04$ & $3.882 \mathrm{e}-01$ & 63.855 & 72.320 & 157.256
\end{tabular}




\section{1-F}

\section{Input orientations}

The RDCs of run 1-F were calculated by adding random Gaussian error to the RDCs of 1-C (for more information see the start of section 2). Therefore the orientation data are reported in table S-14.

\section{Output orientations}

All orientiations of run 1-F (11-39) obtained from the full TITANIA optimization runs are listed in the following section. Changes of the Euler angles compared to the literature data listed above are due to the change of the reference frames (TITANIA used the principle axis system of the molecule) and the added errors. Of high impact for this setup is the missing convergence and thereby large distortions of the structure. This leads to wrong orientation parameters. The comparison of the input and output data is summarized in table S-40 (section 2.2.4).

Table S-30: Orientational data of IPC 1 obtained by TITANIA on the last optimization iteration for the runs 1-F11.

\begin{tabular}{l|ccrcc} 
Set & $\boldsymbol{A}_{\mathrm{zz}}$ & \multicolumn{1}{c}{$\boldsymbol{\alpha}$} & \multicolumn{1}{c}{$\boldsymbol{\alpha}{ }^{\circ}$} & \multicolumn{1}{c}{$\boldsymbol{\beta}{ }^{\circ}$} & \multicolumn{1}{c}{$\boldsymbol{\gamma}{ }^{\circ}$} \\
\hline set 1 & $-7.323 \mathrm{e}-04$ & $1.757 \mathrm{e}-01$ & 21.959 & 53.078 & 131.176 \\
set 2 & $-7.112 \mathrm{e}-04$ & $5.412 \mathrm{e}-01$ & 9.253 & 46.213 & 103.567 \\
set 7 & $-6.834 \mathrm{e}-04$ & $2.104 \mathrm{e}-01$ & 134.133 & 64.583 & 133.945 \\
set 8 & $-7.553 \mathrm{e}-04$ & $5.281 \mathrm{e}-01$ & 10.523 & 32.605 & 100.127 \\
set 9 & $-7.792 \mathrm{e}-04$ & $6.015 \mathrm{e}-01$ & 14.527 & 36.754 & 103.745 \\
set 10 & $-7.135 \mathrm{e}-04$ & $4.771 \mathrm{e}-01$ & 8.645 & 42.312 & 105.833
\end{tabular}

Table S-31: Orientational data of IPC 1 obtained by TITANIA on the last optimization iteration of run 1-F17.

\begin{tabular}{l|rcrrr} 
Set & \multicolumn{1}{|c}{$\boldsymbol{A}_{\mathrm{zz}}$} & \multicolumn{1}{c}{$\boldsymbol{c} \boldsymbol{\alpha} /{ }^{\circ}$} & \multicolumn{1}{c}{$\boldsymbol{\beta} /{ }^{\circ}$} & \multicolumn{1}{c}{$\boldsymbol{\gamma} /^{\circ}$} \\
\hline set 1 & $-5.315 \mathrm{e}-04$ & $4.740 \mathrm{e}-01$ & 108.017 & 51.100 & 147.127 \\
set 2 & $6.644 \mathrm{e}-04$ & $3.148 \mathrm{e}-01$ & 56.389 & 64.107 & 0.762 \\
set 7 & $-5.094 \mathrm{e}-04$ & $6.181 \mathrm{e}-01$ & 114.681 & 59.992 & 160.917 \\
set 8 & $6.937 \mathrm{e}-04$ & $4.115 \mathrm{e}-01$ & 47.726 & 69.585 & 3.639 \\
set 9 & $7.393 \mathrm{e}-04$ & $4.202 \mathrm{e}-01$ & 61.175 & 66.555 & 2.115 \\
set 10 & $6.356 \mathrm{e}-04$ & $4.076 \mathrm{e}-01$ & 54.700 & 64.377 & 0.618
\end{tabular}


Table S-32: Orientational data of IPC 1 obtained by TITANIA on the last optimization iteration of run 1-F23.

\begin{tabular}{l|ccccc} 
Set & $\boldsymbol{A}_{\mathrm{zz}}$ & $\boldsymbol{R}$ & $\boldsymbol{\alpha} /{ }^{\circ}$ & $\boldsymbol{\beta} /{ }^{\circ}$ & $\boldsymbol{\gamma} /{ }^{\circ}$ \\
\hline set 1 & $1.595 \mathrm{e}-03$ & $2.485 \mathrm{e}-01$ & 28.009 & 54.848 & 28.371 \\
set 2 & $2.116 \mathrm{e}-03$ & $1.006 \mathrm{e}-01$ & 23.912 & 61.662 & 12.787 \\
set 7 & $1.510 \mathrm{e}-03$ & $2.810 \mathrm{e}-01$ & 18.880 & 53.793 & 40.299 \\
set 8 & $2.033 \mathrm{e}-03$ & $1.485 \mathrm{e}-01$ & 24.423 & 64.106 & 11.306 \\
set 9 & $2.362 \mathrm{e}-03$ & $1.640 \mathrm{e}-01$ & 21.980 & 63.071 & 14.331 \\
set 10 & $1.989 \mathrm{e}-03$ & $1.162 \mathrm{e}-01$ & 20.411 & 61.822 & 13.466
\end{tabular}

Table S-33: Orientational data of IPC 1 obtained by TITANIA on the last optimization iteration of run 1-F31.

\begin{tabular}{l|rcrrr} 
Set & \multicolumn{1}{|c}{$\boldsymbol{A}_{\mathrm{zz}}$} & \multicolumn{1}{c}{$\boldsymbol{\alpha}$} & \multicolumn{1}{c}{${ }^{\circ}$} & \multicolumn{1}{c}{$\boldsymbol{\beta} /{ }^{\circ}$} & \multicolumn{1}{c}{$\boldsymbol{\gamma} /{ }^{\circ}$} \\
\hline set 1 & $-6.765 \mathrm{e}-04$ & $4.767 \mathrm{e}-01$ & 87.261 & 37.011 & 148.785 \\
set 2 & $7.922 \mathrm{e}-04$ & $5.650 \mathrm{e}-01$ & 106.172 & 110.947 & 177.261 \\
set 7 & $-5.425 \mathrm{e}-04$ & $4.219 \mathrm{e}-01$ & 79.068 & 48.417 & 169.225 \\
set 8 & $8.220 \mathrm{e}-04$ & $6.492 \mathrm{e}-01$ & 67.184 & 74.229 & 0.356 \\
set 9 & $8.507 \mathrm{e}-04$ & $6.143 \mathrm{e}-01$ & 106.840 & 108.146 & 176.660 \\
set 10 & $7.558 \mathrm{e}-04$ & $6.047 \mathrm{e}-01$ & 111.864 & 109.909 & 177.867
\end{tabular}

Table S-34: Orientational data of IPC 1 obtained by TITANIA on the last optimization iteration of run 1-F39.

\begin{tabular}{l|rcrrr} 
Set & \multicolumn{1}{|c}{$\boldsymbol{A}_{\text {zz }}$} & \multicolumn{1}{c}{$\boldsymbol{R}$} & \multicolumn{1}{c}{$\boldsymbol{\alpha} /{ }^{\circ}$} & \multicolumn{1}{c}{$\boldsymbol{\beta}{ }^{\circ}$} & \multicolumn{1}{c}{$\boldsymbol{\gamma}{ }^{\circ}$} \\
\hline set 1 & $-7.593 \mathrm{e}-04$ & $4.385 \mathrm{e}-01$ & 156.619 & 68.190 & 122.764 \\
set 2 & $9.258 \mathrm{e}-04$ & $3.843 \mathrm{e}-01$ & 24.901 & 86.472 & 21.230 \\
set 7 & $-5.855 \mathrm{e}-04$ & $6.374 \mathrm{e}-01$ & 162.446 & 64.609 & 138.184 \\
set 8 & $9.469 \mathrm{e}-04$ & $5.005 \mathrm{e}-01$ & 21.550 & 90.237 & 16.287 \\
set 9 & $9.947 \mathrm{e}-04$ & $4.520 \mathrm{e}-01$ & 27.344 & 88.479 & 18.041 \\
set 10 & $8.596 \mathrm{e}-04$ & $4.968 \mathrm{e}-01$ & 18.928 & 90.301 & 19.966
\end{tabular}




\subsubsection{Optimization Trajectories}

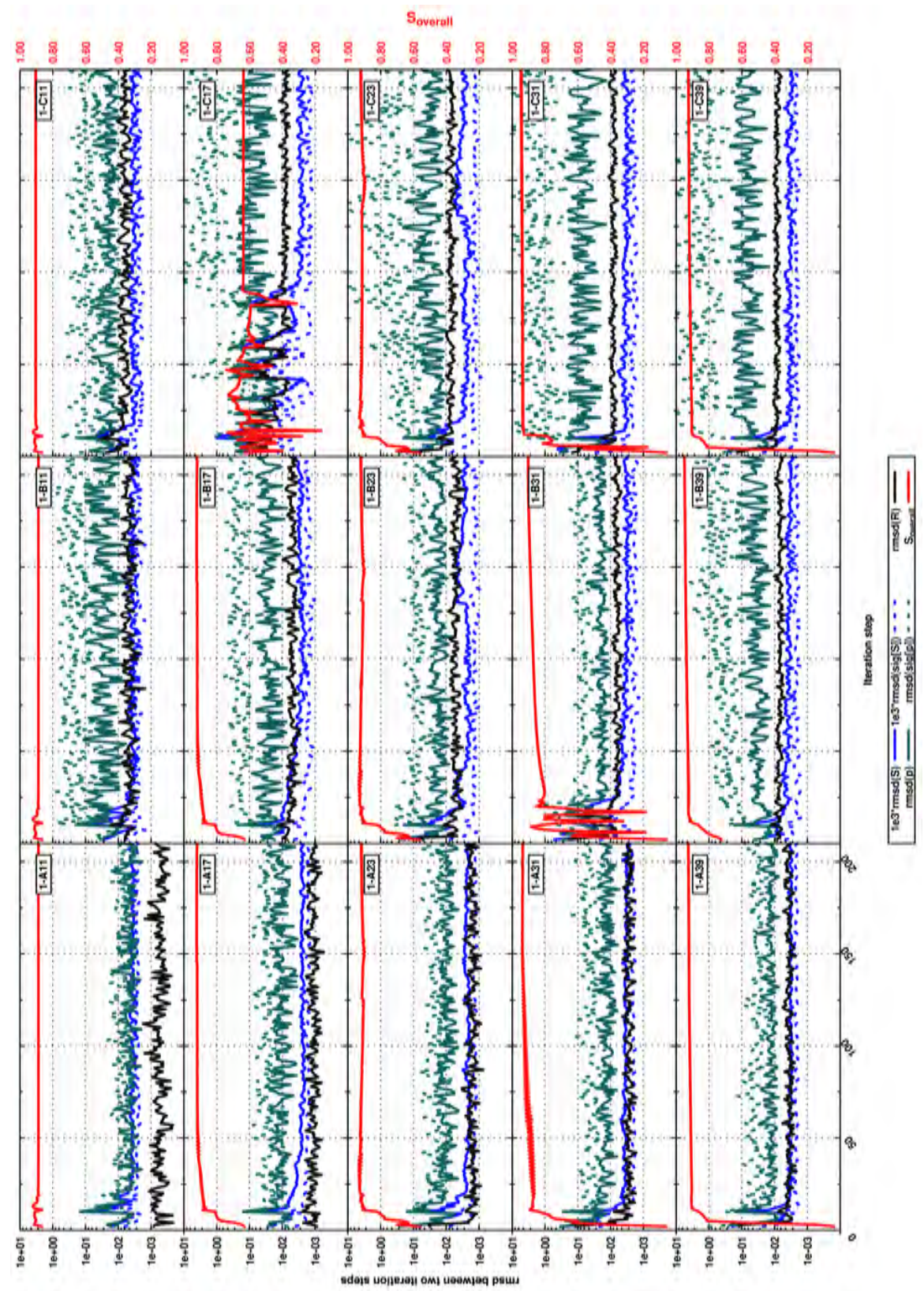

Figure S-10: Trajectory of the Monte-Carlo $\mathrm{rmsds}$ obtained for the individual runs of the setups $\mathbf{1 - A}, \mathbf{B}$ and $\mathbf{C}$. Shown are the data obtained by the default redundant internal coordinates algorithm. 


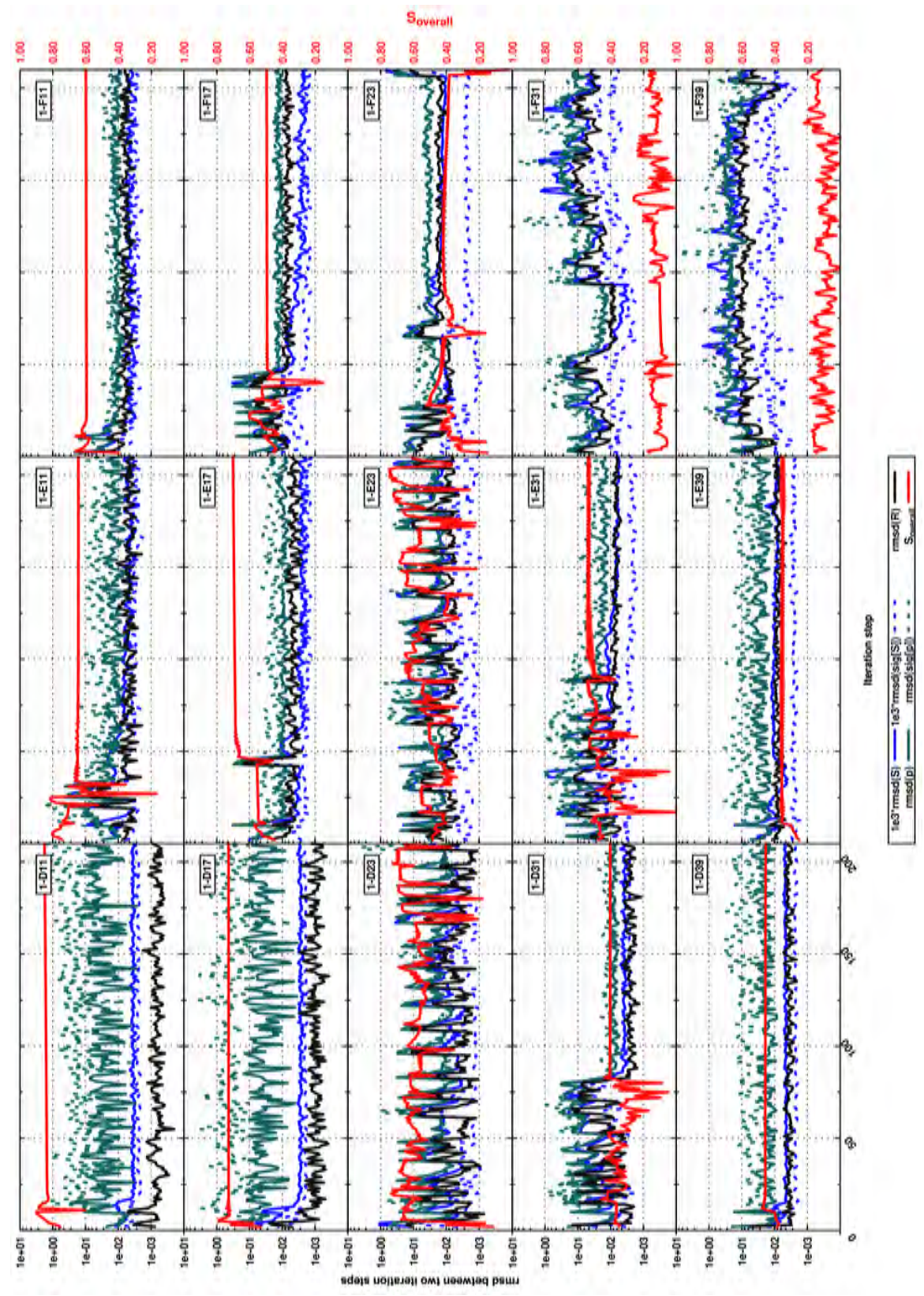

Figure S-11: Trajectory of the Monte-Carlo rmsds obtained for the individual runs of the setups $\mathbf{1 - D}, \mathbf{E}$ and $\mathbf{F}$. Shown are the data obtained by the default redundant internal coordinates algorithm. 


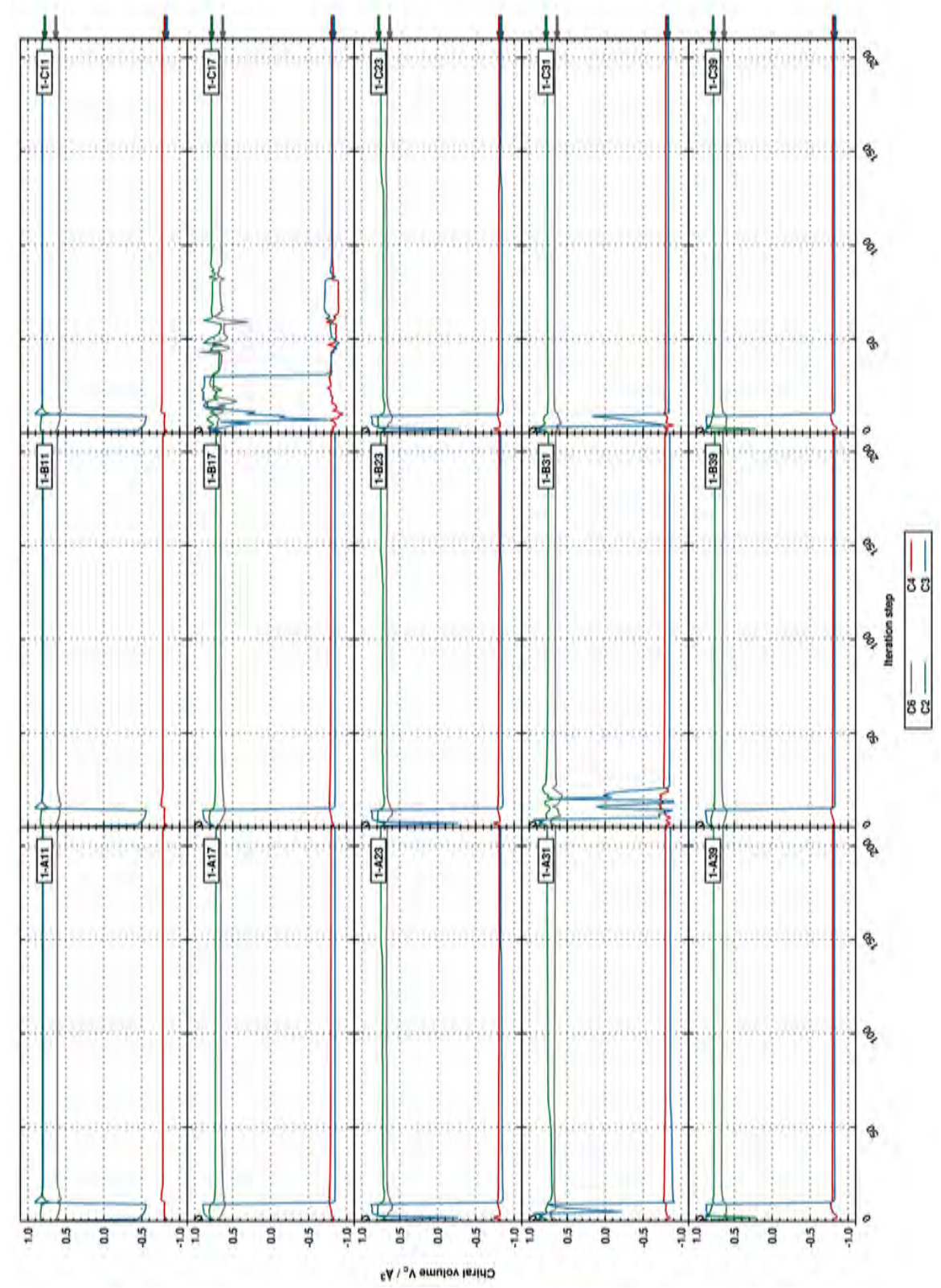

Figure S-12: Trajectory of the normalized chiral volumes obtained for the individual runs of the setups $\mathbf{1 - A}, \mathbf{B}$ and $\mathbf{C}$. Shown are the data obtained by the default redundant internal coordinates algorithm. The arrows at the top indicate the values of the reference structure (correct configuration). 


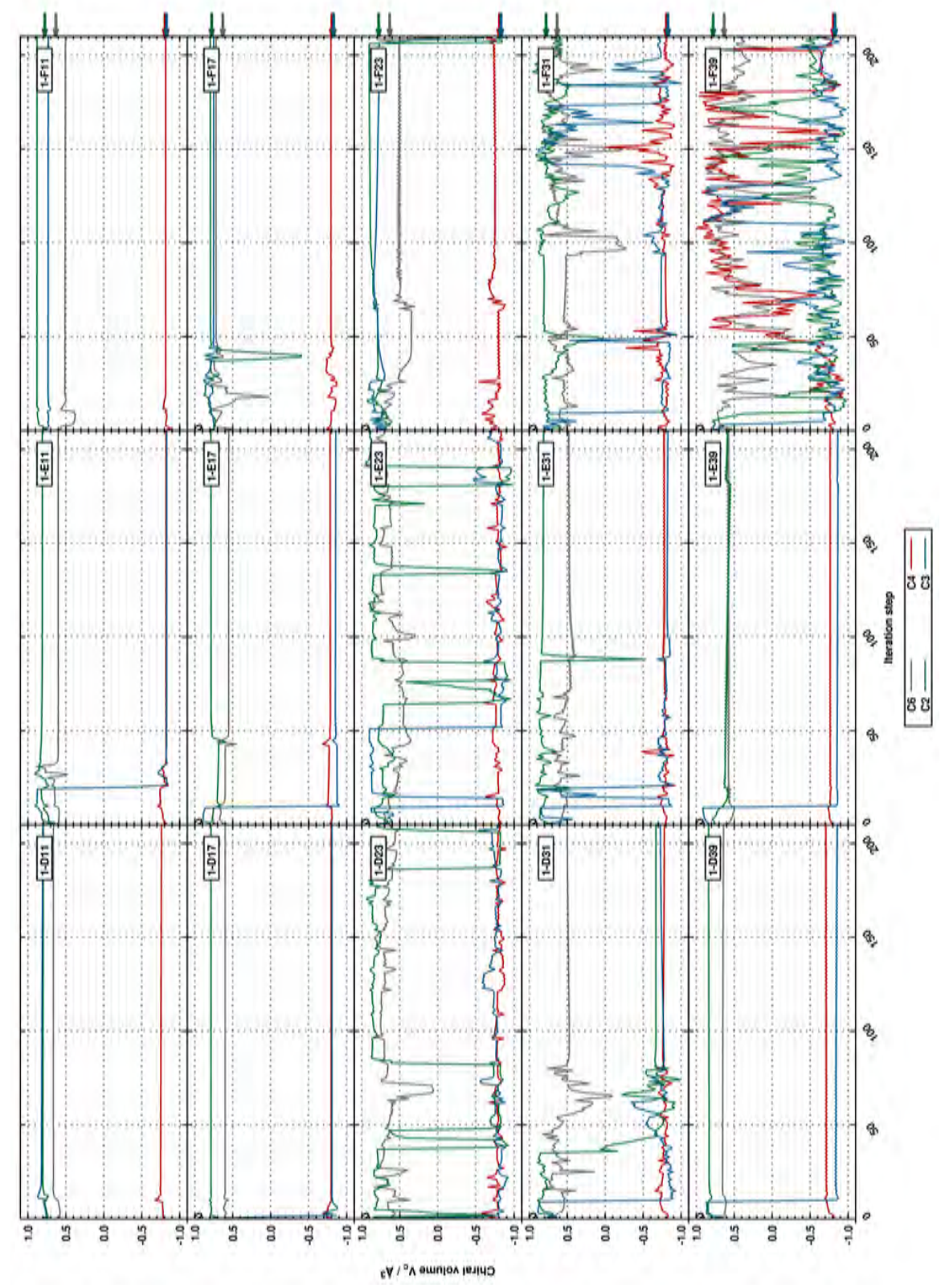

Figure S-13: Trajectory of the normalized chiral volumes obtained for the individual runs of the setups $\mathbf{1 - D}, \mathbf{E}$ and $\mathbf{F}$. Shown are the data obtained by the default redundant internal coordinates algorithm. The arrows at the top indicate the values of the reference structure (correct configuration). 
The iterative progression of TITANIA is assessed by a Monte-Carlo bootstrap after every iteration step. In this bootstrap all RDCs are varied simultaneously by normal distributed random numbers, with the user estimated experimental errors of the individual RDCs as standard deviation, and used for the determination of the alignment (tensor shape and Euler angles) and from that the resulting structure parameters (spherical harmonics and spherical coordinates). The parameters monitored (which can be used as stop criteria, for respective flags see section 1.2.1) are the change (rmsd) in the reduced Saupe matrix $\left(\mathrm{S}_{\mathrm{zz}}, \mathrm{S}_{\mathrm{xx}}-\mathrm{S}_{\mathrm{yy}}, \mathrm{S}_{\mathrm{xy}}, \mathrm{S}_{\mathrm{xz}}, \mathrm{S}_{\mathrm{yz}}\right)$, the trigonometric functions of the polar angles $p$ (e.g. $\cos (\theta))$ and the standard deviation of the respective values. The fifth parameter monitored is the change of the mean length $R_{s}$ of the averaged Monte-Carlo RDC direction vectors (see eq. (34)). In the present runs the thresholds for the convergence were set to zero to show the full trajectories of the runs 1-A to $\mathbf{1 - F}$ (stop criteria not used).

Of special interest in fig. S-10 is the behavior of the structure related parameters. As expected the rmsd of $R_{\mathrm{s}}$ shows less fluctuation when increasing the number of RDCs per set. If convergence is not achieved large spikes in the Monte-Carlo trajectories (see first steps in fig. S-10) and significant changes in the chiral volumes throughout the whole optimization would be observed. All trajectories except 1-C17 show the last significant spike in $S_{\text {overall }}$ around iteration 10, which is the point at which the final configurations are reached. As expected the trajectories $\mathbf{1 - X} \mathbf{1 7}$ to $\mathbf{1 - X 3 9}$ (with $\mathbf{X}$ equals $\mathbf{A}$ to $\mathbf{C}$ ) clearly show convergence to the correct structure. Convergence is also achieved in $\mathbf{1 - A 1 1}$, but the wrong configuration is obtained with a distorted geometry at the center C3.

Compared to the Monte-Carlo plots of setup 1-A the runs of $\mathbf{1 - B}$ and 1-C show larger fluctuations in the parameters monitored. This is due to the fact that here fewer RDC sets are used. $S_{\text {overall }}$ is not as strongly affected by the number of sets as the Monte-Carlo results, as it is determined from the experimental RDCs instead of the Monte-Carlo bootstrap. Only 1-C17 seems to show an unexpected behavior due to a rather low $S_{\text {overall }}$ parameter and high fluctuations in the orientations of the RDC vector obtained from the Monte-Carlo bootstrap ( $r m s d[p]$ and $\operatorname{rmsd}(\operatorname{sig}[p]))$.

These instabilities are based on the linear dependence and the number of RDC sets. This can be seen in the comparison of all runs in fig. S-10. Here the fluctuations of the vector orientations increases when reducing the number of sets (1-A to 1-B) and again when increasing the linear dependence of the sets $(\mathbf{1}-\mathbf{B}$ to $\mathbf{1}-\mathbf{C})$.

This trend seems to change when adding heterogeneity. Here $\mathbf{1 - F}$ shows fast convergence with comparably low $S_{\text {overall values. By investigation of the }}$ geometries it becomes clear that this a random local minimum. This will be 
discussed in more detail on the chiral volume plots. The two last runs (1-F31 and 39) in fig. S-11 show instabilities in the full course of the optimization. This is a hint towards random inversions. This will also be discussed below.

The runs with 11 and 17 RDCs for 1-D and 1-E show an increase in the fluctuations compared to the homogeneous data, with 1-D being more affected. The reason might be the large variety of axial components for 1-D (see table S-41 for more details). This fluctuation is reduced with the number of RDCs, demonstrating that larger set sizes lead to a higher error tolerance. An exception are the runs using 23 RDC, which show no convergence at all. A close look on the xyz coordinates (see supplementary materials section 5) and the chiral volume trajectories allows a better assessment of these two runs. By this it becomes clear that 1-E23 undergoes several inversions of different centers accompanied by large distortions of the geometry (including large variations in the bond lengths of the ring scaffold). 1-D23 on the other hand only shows large fluctuations at C6 which influences the whole trajectory, especially the center $\mathrm{C} 2$. $\mathrm{C} 2$ and $\mathrm{C} 6$ have in common that ${ }^{1} D_{\mathrm{CC}}$ couplings are used to define the configuration. These are lower in magnitude than ${ }^{1} D_{\mathrm{CH}}$ RDCs. Furthermore no long-range RDCs are available to further define or confirm the configuration. Additionally the $S_{\text {overall }}$ is reduced in size, especially when long-range RDCs are added in the larger setups with 31 or 39 RDCs.

The trajectories of the $\mathbf{1 - F}$ runs with 11 to 23 RDCs imply a fast convergence. This behavior is due to the convergence into a local minimum barely optimizing the structure at all (see below). The runs 1-F31 and 1-F39 show the other scenario where no convergence is achieved at all. Here only random inversions occur during the optimization. Additionally the lowest $S_{\text {overall }}$ for IPC are found in these runs. This shows that the MFA is neither capable to determine structure parameters for heterogeneous, dependent alignment conditions nor to properly determine any dynamic parameters in this scenario.

The chiral volume plots of the homogeneous data (fig. S-12) show, as expected from the main text discussion, very stable configurations. Therefore the trajectories of the heterogeneous setups $\mathbf{1 - D}$ to $\mathbf{1 - F}$ are of larger interest. It is noteworthy that 1-E11 converged into the correct relative configuration. This is unexpected and should not be over-interpreted. More important is that the setups with long-range couplings show a reduction in stability compared to the homogeneous data while still achieving the correct relative configuration. An exception are the runs using 23 RDCs. The trajectories of both the chiral volume and the rmsds shown above, are not stable at any point of the optimization. In such a case the number of RDCs per set should be varied (in a real scenario lowered) or the vector addition algorithm should 
be used. The reduction of the number of RDCs would allows to identify RDCs that disturb the trajectories, most likely due to high local heterogeneity. The alternative vector addition algorithm is more robust and thereby could help to find the correct configurations. This algorithm is discussed later for the heterogeneous setups.

The setup 1-F shows very interesting results. Here the first three trajectories converge very fast without a change in any configurations. If the set size is increased further (1-F31 and 39) rapid inversion without any pattern can be observed. This demonstrates how linear dependence in combination with heterogeneity does not allow to obtain correct vector orientations by using TITANIA.

\subsubsection{Change in Orientation}

To quantify the similarity of the input and the output orientations a common reference frame has to be used. Therefore an all atom rmsd structure alignment was performed to match the TITANIA output structure with the reference structure (or its enantiomer). The alignment tensors of the transformed structures are recalculated and the differences in the orientations (TITANIA vs. reference) are expressed by the $\beta$ angle. 


\section{1-A}

Table S-35: Generalized angle $\beta$ enclosed by the reference structure of IPC and the structure obtained by TITANIA from the last optimization step of the runs 1-A. The data were obtained by transformation of the respective structures to a common reference frame, followed by recalculating the alignment tensors with the in-house software RDC@hotFCHT. ${ }^{[9]}$

\begin{tabular}{l|rrrrr} 
& \multicolumn{5}{|c}{$\boldsymbol{\beta}$ / $^{\circ}$} \\
Set & $\mathbf{1 - A 1 1}$ & $\mathbf{1 - A 1 7}$ & $\mathbf{1 - A} \mathbf{2 3}$ & $\mathbf{1 - A 3 1}$ & $\mathbf{1 - A 3 9}$ \\
\hline set 1 & 5.923 & 3.185 & 2.991 & 5.292 & 1.331 \\
set 2 & 8.151 & 1.968 & 2.981 & 5.732 & 1.067 \\
set 3 & 3.245 & 2.592 & 0.915 & 4.253 & 1.597 \\
set 4 & 9.843 & 2.437 & 1.429 & 2.718 & 0.754 \\
set 5 & 12.561 & 2.022 & 1.855 & 3.214 & 1.393 \\
set 6 & 11.638 & 2.107 & 2.136 & 4.630 & 0.637 \\
set 7 & 8.578 & 1.787 & 1.701 & 5.329 & 0.848 \\
set 8 & 5.394 & 3.385 & 3.354 & 3.429 & 1.138 \\
set 9 & 8.501 & 2.442 & 2.134 & 4.568 & 0.714 \\
set 10 & 5.250 & 3.403 & 2.512 & 4.475 & 1.362 \\
set 11 & 7.617 & 1.904 & 1.687 & 3.285 & 0.802 \\
set 12 & 11.348 & 1.805 & 2.673 & 2.424 & 1.115 \\
set 13 & 9.771 & 2.135 & 1.961 & 4.408 & 0.776 \\
set 14 & 6.866 & 3.345 & 2.227 & 5.294 & 0.604 \\
set 15 & 8.051 & 3.053 & 2.651 & 5.344 & 1.000 \\
set 16 & 7.947 & 2.034 & 2.853 & 5.279 & 1.558 \\
set 17 & 7.454 & 2.524 & 1.594 & 3.482 & 0.716 \\
set 18 & 4.324 & 1.631 & 1.313 & 5.337 & 0.731 \\
set 19 & 6.208 & 2.596 & 3.187 & 3.588 & 1.730 \\
set 20 & 7.094 & 2.881 & 2.136 & 5.383 & 0.438
\end{tabular}

It can be seen that only $\mathbf{1 - A 1 1}$ shows $\beta$ angles of larger magnitude. The reason is the incorrect configuration on C3. All other runs only have very small deviations between the reference and output structures. This confirms the agreement of the TITANIA structure and the reference. 


\section{1-B}

Table S-36: Generalized angle $\beta$ enclosed by the reference structure of IPC and the structure obtained by TITANIA from the last optimization step of the runs 1-B. The data were obtained by transformation of the respective structures to a common reference frame, followed by recalculating the alignment tensors with the in-house software RDC@hotFCHT. ${ }^{[9]}$

\begin{tabular}{l|rrrrr} 
& \multicolumn{5}{|c}{$\boldsymbol{\beta} /{ }^{\circ}$} \\
Set & 1-B11 & 1-B17 & 1-B23 & 1-B31 & 1-B39 \\
\hline set 1 & 2.994 & 2.709 & 2.424 & 2.520 & 2.034 \\
set 2 & 5.193 & 2.339 & 2.763 & 2.234 & 1.953 \\
set 3 & 7.820 & 1.842 & 2.553 & 4.310 & 2.417 \\
set 4 & 7.238 & 1.254 & 2.075 & 3.899 & 2.373 \\
set 5 & 6.125 & 2.185 & 1.767 & 3.641 & 2.174 \\
set 6 & 6.159 & 2.707 & 3.605 & 3.559 & 2.338
\end{tabular}

All runs of the setup 1-B show excellent agreement between the TITANIA and reference structure.

\section{$1-\mathrm{C}$}

Table S-37: Generalized angle $\beta$ enclosed by the reference structure of IPC and the structure obtained by TITANIA from the last optimization step of the runs 1-C. The data were obtained by transformation of the respective structures to a common reference frame, followed by recalculating the alignment tensors with the in-house software RDC@hotFCHT. ${ }^{[9]}$

\begin{tabular}{l|rrrrr} 
& \multicolumn{5}{|c}{$\boldsymbol{\beta} /{ }^{\circ}$} \\
Set & 1-C11 & 1-C17 & 1-C23 & 1-C31 & 1-C39 \\
\hline set 1 & 2.418 & 10.222 & 2.362 & 1.492 & 0.611 \\
set 2 & 4.152 & 20.642 & 2.716 & 1.466 & 1.028 \\
set 7 & 2.550 & 10.907 & 2.679 & 1.669 & 0.715 \\
set 8 & 4.793 & 23.999 & 2.816 & 1.463 & 1.129 \\
set 9 & 4.662 & 23.865 & 2.699 & 1.377 & 1.023 \\
set 10 & 4.029 & 20.034 & 2.702 & 1.457 & 1.021
\end{tabular}

The high $\beta$ angle found for 1-C17 shows a large value due to distortions of the methyl vectors $\mathrm{C} 6-\mathrm{C} 8$ and $\mathrm{C} 6-\mathrm{C} 9$. 


\section{$1-\mathrm{D}$}

Table S-38: Generalized angle $\beta$ enclosed by the reference structure of IPC and the structure obtained by TITANIA from the last optimization step of the runs 1-D. The data were obtained by transformation of the respective structures to a common reference frame, followed by recalculating the alignment tensors with the in-house software RDC@hotFCHT. ${ }^{[9]}$ The mean contribution of the random noise to the $\beta$ angles is reported in table S- 41 .

\begin{tabular}{l|rrrrr} 
& \multicolumn{5}{|c}{$\boldsymbol{\beta}$ / $^{\circ}$} \\
Set & 1-D11 & $\mathbf{1 - D 1 7}$ & $\mathbf{1 - D 2 3}$ & $\mathbf{1 - D 3 1}$ & $\mathbf{1 - D 3 9}$ \\
\hline set 1 & 10.116 & 7.150 & 5.541 & 39.437 & 15.202 \\
set 2 & 13.430 & 16.208 & 14.208 & 45.302 & 13.448 \\
set 3 & 11.460 & 12.745 & 10.916 & 27.275 & 15.252 \\
set 4 & 9.075 & 9.924 & 4.270 & 58.423 & 12.129 \\
set 5 & 5.162 & 10.557 & 7.233 & 57.837 & 8.629 \\
set 6 & 12.618 & 4.166 & 9.068 & 57.086 & 9.181 \\
set 7 & 9.845 & 7.009 & 4.444 & 57.993 & 16.812 \\
set 8 & 11.790 & 4.946 & 10.780 & 32.610 & 13.318 \\
set 9 & 11.158 & 11.279 & 17.303 & 52.909 & 16.708 \\
set 10 & 6.207 & 7.644 & 5.606 & 44.127 & 13.986 \\
set 11 & 14.393 & 5.905 & 4.101 & 52.062 & 8.597 \\
set 12 & 8.996 & 7.016 & 9.430 & 39.717 & 10.459 \\
set 13 & 9.181 & 9.410 & 7.701 & 42.717 & 8.083 \\
set 14 & 7.418 & 15.582 & 12.608 & 47.711 & 10.091 \\
set 15 & 9.178 & 8.688 & 4.663 & 52.551 & 15.287 \\
set 16 & 6.976 & 5.430 & 9.705 & 33.347 & 15.209 \\
set 17 & 13.728 & 9.042 & 4.330 & 60.949 & 10.506 \\
set 18 & 7.528 & 7.684 & 3.738 & 40.481 & 15.600 \\
set 19 & 7.279 & 4.436 & 4.824 & 31.517 & 14.840 \\
set 20 & 10.886 & 7.651 & 7.978 & 51.570 & 9.160
\end{tabular}

Apart from 1-D31, which did not converge using redundant internal coordinates, the $\beta$ angles show good agreement. The values are a combination of the errors added and the deviations in the final compared to the reference structure. The separation of these two effects is addressed later. 


\section{$1-\mathrm{E}$}

Table S-39: Generalized angle $\beta$ enclosed by the reference structure of IPC and the structure obtained by TITANIA from the last optimization step of the runs 1-E. The data were obtained by transformation of the respective structures to a common reference frame, followed by recalculating the alignment tensors with the in-house software RDC@hotFCHT. ${ }^{[9]}$ The mean contribution of the random noise to the $\beta$ angles is reported in table S- 42 .

\begin{tabular}{l|rrrrr} 
& \multicolumn{5}{|c}{$\boldsymbol{\beta} /{ }^{\circ}$} \\
Set & 1-E11 & 1-E17 & 1-E23 & 1-E31 & 1-E39 \\
\hline set 1 & 1.031 & 5.365 & 12.059 & 4.732 & 11.162 \\
set 2 & 8.733 & 2.112 & 10.428 & 2.994 & 11.562 \\
set 3 & 12.759 & 8.271 & 3.496 & 7.317 & 14.976 \\
set 4 & 13.415 & 5.349 & 6.096 & 4.400 & 16.701 \\
set 5 & 13.848 & 6.355 & 4.570 & 4.474 & 16.945 \\
set 6 & 11.343 & 8.382 & 4.536 & 10.023 & 7.351
\end{tabular}

The $\beta$ angles are a combination of the errors added and the deviations in the final compared to the reference structure. The separation of this two effects is addressed later.

\section{$1-\mathrm{F}$}

Table S-40: Generalized angle $\beta$ enclosed by the reference structure of IPC and the structure obtained by TITANIA from the last optimization step of the runs $\mathbf{1 - F}$. The data were obtained by transformation of the respective structures to a common reference frame, followed by recalculating the alignment tensors with the in-house software RDC@hotFCHT. ${ }^{[9]}$ The mean contribution of the random noise to the $\beta$ angles is reported in table S- 43 .

\begin{tabular}{l|rrrrr} 
& \multicolumn{5}{|c}{$\boldsymbol{\beta} /{ }^{\circ}$} \\
Set & 1-F11 & 1-F17 & $\mathbf{1 - F 2 3}$ & 1-F31 & 1-F39 \\
\hline set 1 & 37.217 & 22.950 & 103.611 & 57.157 & 55.099 \\
set 2 & 58.962 & 19.781 & 108.086 & 58.470 & 55.278 \\
set 7 & 36.418 & 25.518 & 115.432 & 63.649 & 61.063 \\
set 8 & 51.858 & 28.486 & 104.242 & 63.774 & 56.893 \\
set 9 & 58.467 & 21.751 & 103.605 & 60.611 & 55.627 \\
set 10 & 55.075 & 21.936 & 105.504 & 60.508 & 58.774
\end{tabular}

In the setup 1-F the error now shows the importance of linear independence in the alignment media when experimental error is present. None of 
the runs shown has converged.

\subsubsection{Change induced by error}

When adding error to the RDCs a change in the orientation is induced. This change is addressed in this section. In the following tables the generalized $\beta$ angle $\beta$ is calculated for the individual sets. Since a change in the orientation can be induced by the change of RDCs as well as by structural changes the following $\beta$ angles contain this information simultaneously. To address the pure change due to the variation of RDCs, the mean $\beta$ angle (and the corresponding standard deviation) of the individual runs is calculated using the reference structure.

\section{1-A}

Some $\beta$-angles in the $\mathbf{1 - A}$ vs. 1-D comparison have rather large magnitudes. This is especially true for the sets with small $A_{\mathrm{zz}}$ values (see table S-2). Therefore the rows of table $\mathrm{S}-41$ have a grayscale filling according to their $A_{\mathrm{zz}}$ value with smaller values being brighter and larger values being darker. 
Table S-41: Generalized angle $\beta$ enclosed by the orientations of IPC 1 obtained by TITANIA in the last optimization step of the runs $\mathbf{1 - A}$ and $\mathbf{1 - D}$. The last column contains the input $A_{\mathrm{zz}}$ values. Since the alignment parameters (see table S-2) were generated from random numbers some of the $A_{\mathrm{zz}}$ values are much lower than others. This in combination with the artificial error added in setup 1-D to larger deviations compared to the results of 1A. Therefore the grayscale of the rows are used for faster recognition of the respective $A_{\mathrm{zz}}$ (brighter: lower value).

\begin{tabular}{lrrrrrr} 
& \multicolumn{7}{|c}{$\boldsymbol{\beta} \boldsymbol{}^{\circ}$} \\
Set & $\mathbf{1 - X 1 1}$ & $\mathbf{1 - X 1 7}$ & $\mathbf{1 - X 2 3}$ & $\mathbf{1 - X 3 1}$ & $\mathbf{1 - X 3 9}$ & $\boldsymbol{A}_{\mathbf{z z}}$ \\
\hline set 1 & 13.754 & 8.389 & 4.391 & 37.700 & 8.956 & $6.502 \mathrm{e}-04$ \\
\hline set 2 & 56.361 & 24.603 & 13.036 & 73.498 & 52.239 & $8.426 \mathrm{e}-05$ \\
set 3 & 45.596 & 19.352 & 21.619 & 31.226 & 40.387 & $8.788 \mathrm{e}-05$ \\
\hline set 4 & 7.884 & 11.785 & 12.640 & 58.093 & 15.019 & $4.350 \mathrm{e}-04$ \\
set 5 & 9.210 & 15.631 & 4.966 & 62.720 & 18.800 & $3.411 \mathrm{e}-04$ \\
\hline set 6 & 7.223 & 5.349 & 10.551 & 57.126 & 7.043 & $8.659 \mathrm{e}-04$ \\
set 7 & 7.014 & 4.296 & 6.884 & 58.397 & 16.848 & $8.074 \mathrm{e}-04$ \\
\hline set 8 & 7.611 & 5.447 & 12.093 & 30.660 & 17.325 & $4.177 \mathrm{e}-04$ \\
\hline set 9 & 62.542 & 42.149 & 48.853 & 103.289 & 66.291 & $4.881 \mathrm{e}-05$ \\
\hline set 10 & 10.053 & 8.561 & 5.996 & 43.642 & 14.567 & $7.993 \mathrm{e}-04$ \\
\hline set 11 & 18.252 & 15.942 & 12.937 & 55.436 & 10.125 & $3.255 \mathrm{e}-04$ \\
set 12 & 18.496 & 9.949 & 3.941 & 39.637 & 19.294 & $4.468 \mathrm{e}-04$ \\
\hline set 13 & 16.268 & 10.687 & 8.240 & 50.801 & 21.353 & $3.196 \mathrm{e}-04$ \\
\hline set 14 & 22.749 & 27.244 & 36.312 & 49.772 & 27.672 & $6.600 \mathrm{e}-05$ \\
\hline set 15 & 13.067 & 5.993 & 7.141 & 53.089 & 14.881 & $7.857 \mathrm{e}-04$ \\
\hline set 16 & 12.608 & 7.207 & 11.477 & 38.565 & 25.334 & $4.146 \mathrm{e}-04$ \\
\hline set 17 & 8.950 & 7.007 & 7.185 & 59.096 & 10.198 & $6.562 \mathrm{e}-04$ \\
set 18 & 6.602 & 6.626 & 4.010 & 40.255 & 23.232 & $5.977 \mathrm{e}-04$ \\
\hline set 19 & 4.636 & 2.425 & 8.698 & 29.789 & 16.583 & $8.809 \mathrm{e}-04$ \\
\hline set 20 & 13.965 & 7.359 & 8.405 & 54.562 & 14.024 & $6.492 \mathrm{e}-04$ \\
\hline$\langle\beta\rangle^{\mathrm{a}}$ & 12.648 & 9.251 & 8.501 & 14.399 & 14.142 & \\
\hline$\sigma$ & 16.649 & 10.110 & 8.877 & 18.626 & 15.591 & \\
\hline
\end{tabular}

a) To separate the impact of the final structure from the variation of the RDCs, the $\beta$ angle was calculated from the reference structure using the RDC sets of setups 1-A and 1-D. The mean angle of all 20 sets (and the respective standard deviation $\sigma$ ) was calculated for the individual set sizes and is reported here. 


\section{1-B}

Table S-42: Generalized angle $\beta$ enclosed by the orientations of IPC $\mathbf{1}$ obtained by TITANIA in the last optimization step of the runs 1-B (see tables S-9 to S-13) and 1-E (see tables S-25 to S-29).

\begin{tabular}{l|rrrrr} 
& \multicolumn{5}{|c}{$\boldsymbol{\beta} /{ }^{\circ}$} \\
Set & $\mathbf{1 - X 1 1}$ & $\mathbf{1 - X 1 7}$ & $\mathbf{1 - X 2 3}$ & $\mathbf{1 - X 3 1}$ & $\mathbf{1 - X 3 9}$ \\
\hline set 1 & 4.969 & 3.238 & 10.145 & 3.410 & 8.860 \\
set 2 & 13.312 & 3.264 & 9.710 & 6.011 & 14.149 \\
set 3 & 16.851 & 10.181 & 5.003 & 7.905 & 17.732 \\
set 4 & 22.522 & 4.413 & 7.169 & 5.435 & 15.439 \\
set 5 & 19.989 & 7.013 & 3.581 & 8.476 & 13.816 \\
set 6 & 11.654 & 8.756 & 4.523 & 11.833 & 5.536 \\
$\langle\beta\rangle^{\mathrm{a})}$ & 2.826 & 2.347 & 2.068 & 3.334 & 4.802 \\
$\sigma$ & 0.961 & 0.863 & 0.516 & 1.371 & 1.628
\end{tabular}

a) To separate the impact of the final structure from the variation of the RDCs, the $\beta$ angle was calculated from the reference structure using the RDC sets of setups 1-B and 1-E. The mean angle of all 6 sets (and the respective standard deviation $\sigma$ ) was calculated for the individual set sizes and is reported here. 


\section{$1-\mathrm{C}$}

Table S-43: Generalized angle $\beta$ enclosed by the orientations of IPC 1 obtained by TITANIA in the last optimization step of the runs $\mathbf{1 - C}$ (see tables S-15 to S-19) and 1-F (see tables S-30 to S-34).

\begin{tabular}{l|rrrrr} 
& \multicolumn{5}{|c}{$\boldsymbol{\beta} \boldsymbol{~}^{\circ}$} \\
Set & $\mathbf{1 - X 1 1}$ & $\mathbf{1 - X 1 7}$ & $\mathbf{1 - X 2 3}$ & $\mathbf{1 - X 3 1}$ & $\mathbf{1 - X 3 9}$ \\
\hline set 1 & 39.620 & 37.102 & 101.492 & 60.392 & 58.817 \\
set 2 & 57.278 & 40.919 & 105.975 & 63.705 & 62.843 \\
set 7 & 37.742 & 41.943 & 114.199 & 64.808 & 63.499 \\
set 8 & 49.962 & 49.684 & 102.378 & 64.122 & 58.861 \\
set 9 & 59.757 & 44.155 & 102.100 & 61.099 & 59.238 \\
set 10 & 54.290 & 38.990 & 104.644 & 62.866 & 58.941 \\
$\langle\beta\rangle^{\text {a) }}$ & 3.329 & 2.416 & 2.070 & 2.868 & 4.384 \\
$\sigma$ & 1.279 & 0.775 & 0.613 & 0.732 & 0.770
\end{tabular}

a) To separate the impact of the final structure from the variation of the RDCs, the $\beta$ angle was calculated from the reference structure using the RDC sets of setups 1-C and 1-F. The mean angle of all 6 sets (and the respective standard deviation $\sigma$ ) was calculated for the individual set sizes and is reported here. 


\subsection{Random coordinates structure}

\subsubsection{Keyword adjustments}

The runs using random coordinates as input structure were adjusted by the TITANIA keywords as follows:

Table S-44: Keywords for the standard IPC runs (using redundant internal coordinates, stan) that changed when using random coordinates as input (rnd \#). Some keywords had to be adjusted for the individual runs to achieve convergence. This is especially true for the damping constants (see rnd 3 and 4 ) and the convergence threshold (rnd 2).

\begin{tabular}{l|rrrrr} 
Keyword & stan & rnd $\mathbf{1}$ & rnd $\mathbf{2}$ & rnd $\mathbf{3}$ & rnd 4 \\
\hline useInitialHolonomics & 1 & 0 & 0 & 0 & 0 \\
MaxTITANIAiterations & 200 & 1000 & 1000 & 1000 & 1000 \\
overoptimizationsteps & 10 & 0 & 0 & 0 & 0 \\
MeanAlignmentConvergence & 0 & $1 \mathrm{e}-6$ & $\mathbf{1 e}-\mathbf{5}$ & $1 \mathrm{e}-6$ & $1 \mathrm{e}-6$ \\
SigmaAlignmentConvergence & 0 & $1 \mathrm{e}-6$ & $1 \mathrm{e}-6$ & $1 \mathrm{e}-6$ & $1 \mathrm{e}-6$ \\
MeanAngleConvergence & 0 & $1 \mathrm{e}-3$ & $1 \mathrm{e}-3$ & $1 \mathrm{e}-3$ & $1 \mathrm{e}-3$ \\
SigmaAngleConvergence & 0 & $1 \mathrm{e}-3$ & $1 \mathrm{e}-3$ & $1 \mathrm{e}-3$ & $1 \mathrm{e}-3$ \\
SpreadAngleConvergence & 0 & $1 \mathrm{e}-5$ & $1 \mathrm{e}-5$ & $1 \mathrm{e}-5$ & $1 \mathrm{e}-5$ \\
useRedundantsOnlyAfter & 10 & 25 & 25 & 25 & 25 \\
redundantsDamping & 0 & 50 & 50 & $\mathbf{5}$ & $\mathbf{5}$ \\
StaticBondWeighting & - & 2.0 & 2.0 & 2.0 & - \\
StaticRDCWeighting & - & 2.0 & 2.0 & 2.0 & - \\
floatingRDCangles & 0 & 1 & 1 & 1 & 1
\end{tabular}

The change in rnd $\mathbf{2}$ was done to prevent instabilities in late iteration steps. When the initial holonomical terms (internal coordinates) cannot be used (no proper initial structure was defined) the MMFF94 equilibrium parameters are used. These do not contain the optimal values for IPC and thereby can lead to instabilities in the late optimization steps. The second changes to the damping / weighting of the redundant internal coordinates (rnd 3/4) are due to slow progression in the structure generation overall. Since no proper input structure is used, the structure generation algorithm needs not only to update the coordinates according to RDC information but also to build a chemically meaningful structure. For some structures this was not possible due to high damping constants. 


\subsubsection{Output orientations}

\section{Isopinocampheol run 1-A}

\section{Random start coordinates}

The orientations when starting from random coordinates are summarized in the tables below. Note that the Euler angles might again be different due to completely different molecular frames as random coordinates are used. An additional change is observed when $(+)$-IPC is obtained instead of the reference structure $((-)$ IPC). These differences are addressed in table S-60 (section 2.2.4).

Table S-45: Orientational data of IPC $\mathbf{1}$ obtained by TITANIA on the last optimization iteration for the runs 1-A11 starting from random coordinates.

\begin{tabular}{l|rcrrr} 
Set & \multicolumn{1}{c}{$\boldsymbol{A}_{\mathrm{zz}}$} & $\boldsymbol{R}$ & \multicolumn{1}{c}{$\boldsymbol{\alpha} /{ }^{\circ}$} & \multicolumn{1}{c}{$\boldsymbol{\beta} /{ }^{\circ}$} & \multicolumn{1}{c}{$\boldsymbol{\gamma} /{ }^{\circ}$} \\
\hline set 1 & $4.839 \mathrm{e}-04$ & $4.592 \mathrm{e}-01$ & 118.016 & 54.440 & 167.509 \\
set 2 & $6.072 \mathrm{e}-05$ & $5.690 \mathrm{e}-01$ & 36.003 & 91.575 & 31.163 \\
set 3 & $8.278 \mathrm{e}-05$ & $3.771 \mathrm{e}-01$ & 89.705 & 46.222 & 60.765 \\
set 4 & $2.986 \mathrm{e}-04$ & $3.379 \mathrm{e}-01$ & 167.382 & 96.818 & 81.982 \\
set 5 & $3.295 \mathrm{e}-04$ & $1.487 \mathrm{e}-01$ & 125.607 & 141.502 & 74.481 \\
set 6 & $7.976 \mathrm{e}-04$ & $3.053 \mathrm{e}-01$ & 82.157 & 115.741 & 151.396 \\
set 7 & $5.172 \mathrm{e}-04$ & $2.636 \mathrm{e}-01$ & 40.703 & 24.521 & 134.680 \\
set 8 & $3.827 \mathrm{e}-04$ & $3.915 \mathrm{e}-01$ & 125.158 & 74.802 & 69.539 \\
set 9 & $4.189 \mathrm{e}-05$ & $2.190 \mathrm{e}-01$ & 50.612 & 101.735 & 124.954 \\
set 10 & $-6.537 \mathrm{e}-04$ & $6.091 \mathrm{e}-01$ & 39.394 & 40.145 & 41.577 \\
set 11 & $3.437 \mathrm{e}-04$ & $5.113 \mathrm{e}-01$ & 101.841 & 79.313 & 111.505 \\
set 12 & $-3.720 \mathrm{e}-04$ & $1.885 \mathrm{e}-01$ & 147.554 & 72.250 & 16.675 \\
set 13 & $-3.539 \mathrm{e}-04$ & $6.249 \mathrm{e}-01$ & 119.260 & 159.058 & 154.246 \\
set 14 & $-5.881 \mathrm{e}-05$ & $5.639 \mathrm{e}-01$ & 82.922 & 150.163 & 136.916 \\
set 15 & $5.238 \mathrm{e}-04$ & $3.291 \mathrm{e}-02$ & 22.832 & 49.074 & 159.015 \\
set 16 & $3.679 \mathrm{e}-04$ & $5.108 \mathrm{e}-01$ & 93.376 & 48.331 & 16.745 \\
set 17 & $5.279 \mathrm{e}-04$ & $2.085 \mathrm{e}-01$ & 77.281 & 91.186 & 99.244 \\
set 18 & $4.404 \mathrm{e}-04$ & $5.078 \mathrm{e}-01$ & 106.080 & 20.323 & 34.049 \\
set 19 & $8.224 \mathrm{e}-04$ & $5.358 \mathrm{e}-02$ & 159.485 & 60.825 & 54.351 \\
set 20 & $-6.506 \mathrm{e}-04$ & $4.306 \mathrm{e}-01$ & 81.562 & 141.746 & 126.907
\end{tabular}


Table S-46: Orientational data of IPC 1 obtained by TITANIA on the last optimization iteration of run 1-A17 starting from random coordinates.

\begin{tabular}{l|ccrrr} 
Set & $\boldsymbol{A}_{\text {zz }}$ & $\boldsymbol{R}$ & \multicolumn{1}{c}{$\boldsymbol{\alpha} /{ }^{\circ}$} & \multicolumn{1}{c}{$\boldsymbol{\beta} /{ }^{\circ}$} & \multicolumn{1}{c}{$\boldsymbol{\gamma} /{ }^{\circ}$} \\
\hline set 1 & $6.954 \mathrm{e}-04$ & $2.925 \mathrm{e}-01$ & 55.852 & 45.461 & 92.973 \\
set 2 & $7.192 \mathrm{e}-05$ & $3.067 \mathrm{e}-01$ & 63.428 & 87.206 & 49.690 \\
set 3 & $8.369 \mathrm{e}-05$ & $3.467 \mathrm{e}-01$ & 88.792 & 136.879 & 32.374 \\
set 4 & $4.394 \mathrm{e}-04$ & $5.280 \mathrm{e}-01$ & 76.282 & 128.625 & 113.355 \\
set 5 & $3.096 \mathrm{e}-04$ & $4.440 \mathrm{e}-01$ & 83.525 & 140.058 & 165.625 \\
set 6 & $8.205 \mathrm{e}-04$ & $3.652 \mathrm{e}-01$ & 100.024 & 84.337 & 171.141 \\
set 7 & $7.679 \mathrm{e}-04$ & $6.009 \mathrm{e}-02$ & 85.965 & 24.599 & 123.780 \\
set 8 & $4.368 \mathrm{e}-04$ & $2.228 \mathrm{e}-01$ & 55.951 & 118.123 & 82.199 \\
set 9 & $4.315 \mathrm{e}-05$ & $3.677 \mathrm{e}-01$ & 127.728 & 99.390 & 148.306 \\
set 10 & $8.236 \mathrm{e}-04$ & $5.626 \mathrm{e}-01$ & 62.944 & 52.580 & 94.645 \\
set 11 & $2.988 \mathrm{e}-04$ & $8.436 \mathrm{e}-02$ & 91.269 & 102.299 & 116.056 \\
set 12 & $3.924 \mathrm{e}-04$ & $4.363 \mathrm{e}-01$ & 18.298 & 138.857 & 140.021 \\
set 13 & $2.906 \mathrm{e}-04$ & $1.559 \mathrm{e}-01$ & 10.538 & 80.777 & 109.112 \\
set 14 & $6.557 \mathrm{e}-05$ & $2.034 \mathrm{e}-01$ & 153.116 & 55.799 & 101.029 \\
set 15 & $8.246 \mathrm{e}-04$ & $9.017 \mathrm{e}-02$ & 79.230 & 38.832 & 103.451 \\
set 16 & $4.197 \mathrm{e}-04$ & $4.848 \mathrm{e}-01$ & 60.351 & 117.667 & 18.093 \\
set 17 & $6.405 \mathrm{e}-04$ & $5.571 \mathrm{e}-01$ & 99.292 & 118.392 & 121.652 \\
set 18 & $5.426 \mathrm{e}-04$ & $3.757 \mathrm{e}-01$ & 175.003 & 34.915 & 174.085 \\
set 19 & $9.052 \mathrm{e}-04$ & $2.190 \mathrm{e}-01$ & 68.135 & 123.906 & 59.326 \\
set 20 & $6.510 \mathrm{e}-04$ & $5.326 \mathrm{e}-01$ & 151.801 & 49.558 & 100.747
\end{tabular}


Table S-47: Orientational data of IPC 1 obtained by TITANIA on the last optimization iteration of run 1-A23 starting from random coordinates.

\begin{tabular}{l|ccrrr} 
Set & $\boldsymbol{A}_{\text {zz }}$ & $\boldsymbol{R}$ & \multicolumn{1}{c}{$\boldsymbol{\alpha} /{ }^{\circ}$} & \multicolumn{1}{c}{$\boldsymbol{\beta} /{ }^{\circ}$} & \multicolumn{1}{c}{$\boldsymbol{\gamma} /{ }^{\circ}$} \\
\hline set 1 & $6.033 \mathrm{e}-04$ & $2.867 \mathrm{e}-01$ & 99.829 & 92.800 & 172.167 \\
set 2 & $5.710 \mathrm{e}-05$ & $3.844 \mathrm{e}-01$ & 47.847 & 49.606 & 30.652 \\
set 3 & $7.409 \mathrm{e}-05$ & $3.771 \mathrm{e}-01$ & 171.832 & 25.581 & 127.866 \\
set 4 & $3.917 \mathrm{e}-04$ & $5.529 \mathrm{e}-01$ & 31.508 & 148.272 & 88.814 \\
set 5 & $2.641 \mathrm{e}-04$ & $4.018 \mathrm{e}-01$ & 31.760 & 115.606 & 54.323 \\
set 6 & $6.652 \mathrm{e}-04$ & $4.241 \mathrm{e}-01$ & 41.383 & 78.696 & 96.816 \\
set 7 & $6.525 \mathrm{e}-04$ & $1.111 \mathrm{e}-01$ & 62.783 & 62.530 & 165.362 \\
set 8 & $4.005 \mathrm{e}-04$ & $2.210 \mathrm{e}-01$ & 83.485 & 162.710 & 138.647 \\
set 9 & $3.439 \mathrm{e}-05$ & $3.918 \mathrm{e}-01$ & 10.578 & 103.737 & 100.210 \\
set 10 & $7.593 \mathrm{e}-04$ & $5.503 \mathrm{e}-01$ & 94.367 & 99.391 & 169.314 \\
set 11 & $2.654 \mathrm{e}-04$ & $1.425 \mathrm{e}-01$ & 50.969 & 131.943 & 125.173 \\
set 12 & $3.195 \mathrm{e}-04$ & $2.656 \mathrm{e}-01$ & 97.557 & 132.151 & 63.327 \\
set 13 & $2.704 \mathrm{e}-04$ & $8.378 \mathrm{e}-02$ & 133.997 & 119.266 & 146.055 \\
set 14 & $5.835 \mathrm{e}-05$ & $1.896 \mathrm{e}-01$ & 6.372 & 101.064 & 162.659 \\
set 15 & $6.978 \mathrm{e}-04$ & $1.381 \mathrm{e}-01$ & 68.681 & 83.013 & 167.492 \\
set 16 & $3.333 \mathrm{e}-04$ & $5.258 \mathrm{e}-01$ & 0.205 & 43.602 & 99.840 \\
set 17 & $5.499 \mathrm{e}-04$ & $5.853 \mathrm{e}-01$ & 25.915 & 138.686 & 101.145 \\
set 18 & $5.014 \mathrm{e}-04$ & $4.104 \mathrm{e}-01$ & 112.684 & 48.102 & 140.910 \\
set 19 & $7.785 \mathrm{e}-04$ & $2.432 \mathrm{e}-01$ & 28.363 & 6.408 & 54.599 \\
set 20 & $5.526 \mathrm{e}-04$ & $5.397 \mathrm{e}-01$ & 4.534 & 95.004 & 164.913
\end{tabular}


Table S-48: Orientational data of IPC 1 obtained by TITANIA on the last optimization iteration of run 1-A31 starting from random coordinates.

\begin{tabular}{l|ccrrr} 
Set & $\boldsymbol{A}_{\text {zz }}$ & $\boldsymbol{R}$ & \multicolumn{1}{c}{$\boldsymbol{\alpha} /{ }^{\circ}$} & \multicolumn{1}{c}{$\boldsymbol{\beta} /{ }^{\circ}$} & \multicolumn{1}{c}{$\boldsymbol{\gamma} /{ }^{\circ}$} \\
\hline set 1 & $5.816 \mathrm{e}-04$ & $2.622 \mathrm{e}-01$ & 128.497 & 140.473 & 127.589 \\
set 2 & $6.657 \mathrm{e}-05$ & $3.357 \mathrm{e}-01$ & 109.515 & 86.475 & 120.877 \\
set 3 & $7.503 \mathrm{e}-05$ & $3.132 \mathrm{e}-01$ & 163.076 & 40.204 & 79.321 \\
set 4 & $4.083 \mathrm{e}-04$ & $5.320 \mathrm{e}-01$ & 99.437 & 89.367 & 48.098 \\
set 5 & $2.866 \mathrm{e}-04$ & $4.141 \mathrm{e}-01$ & 78.630 & 85.431 & 10.624 \\
set 6 & $7.250 \mathrm{e}-04$ & $4.594 \mathrm{e}-01$ & 108.136 & 42.574 & 172.090 \\
set 7 & $7.079 \mathrm{e}-04$ & $7.607 \mathrm{e}-02$ & 149.386 & 24.014 & 1.585 \\
set 8 & $3.996 \mathrm{e}-04$ & $2.401 \mathrm{e}-01$ & 86.601 & 84.302 & 74.384 \\
set 9 & $3.837 \mathrm{e}-05$ & $3.540 \mathrm{e}-01$ & 124.136 & 126.470 & 25.324 \\
set 10 & $7.200 \mathrm{e}-04$ & $5.582 \mathrm{e}-01$ & 126.070 & 137.606 & 115.109 \\
set 11 & $2.834 \mathrm{e}-04$ & $1.036 \mathrm{e}-01$ & 119.228 & 114.256 & 61.285 \\
set 12 & $3.610 \mathrm{e}-04$ & $3.491 \mathrm{e}-01$ & 21.212 & 85.312 & 27.877 \\
set 13 & $2.799 \mathrm{e}-04$ & $9.792 \mathrm{e}-02$ & 32.208 & 127.620 & 79.352 \\
set 14 & $5.843 \mathrm{e}-05$ & $2.693 \mathrm{e}-01$ & 24.481 & 140.351 & 109.510 \\
set 15 & $7.053 \mathrm{e}-04$ & $1.168 \mathrm{e}-01$ & 175.364 & 150.632 & 138.425 \\
set 16 & $3.401 \mathrm{e}-04$ & $4.516 \mathrm{e}-01$ & 112.591 & 39.681 & 118.886 \\
set 17 & $5.858 \mathrm{e}-04$ & $5.259 \mathrm{e}-01$ & 116.788 & 101.720 & 48.082 \\
set 18 & $5.262 \mathrm{e}-04$ & $4.133 \mathrm{e}-01$ & 124.178 & 18.500 & 63.275 \\
set 19 & $7.706 \mathrm{e}-04$ & $1.749 \mathrm{e}-01$ & 108.004 & 66.201 & 82.070 \\
set 20 & $5.686 \mathrm{e}-04$ & $6.139 \mathrm{e}-01$ & 36.801 & 144.694 & 120.895
\end{tabular}


Table S-49: Orientational data of IPC 1 obtained by TITANIA on the last optimization iteration of run 1-A39 starting from random coordinates.

\begin{tabular}{l|ccrrr} 
Set & $\boldsymbol{A}_{\mathrm{zz}}$ & $\boldsymbol{R}$ & \multicolumn{1}{c}{$\boldsymbol{\alpha} /{ }^{\circ}$} & \multicolumn{1}{c}{$\boldsymbol{\beta} /{ }^{\circ}$} & \multicolumn{1}{c}{$\boldsymbol{\gamma} /{ }^{\circ}$} \\
\hline set 1 & $5.891 \mathrm{e}-04$ & $2.767 \mathrm{e}-01$ & 124.469 & 95.506 & 94.653 \\
set 2 & $7.370 \mathrm{e}-05$ & $3.083 \mathrm{e}-01$ & 146.883 & 121.502 & 144.053 \\
set 3 & $8.000 \mathrm{e}-05$ & $3.692 \mathrm{e}-01$ & 9.604 & 41.840 & 41.012 \\
set 4 & $3.734 \mathrm{e}-04$ & $6.173 \mathrm{e}-01$ & 29.536 & 162.189 & 5.752 \\
set 5 & $3.042 \mathrm{e}-04$ & $3.889 \mathrm{e}-01$ & 159.437 & 54.078 & 149.638 \\
set 6 & $7.730 \mathrm{e}-04$ & $4.809 \mathrm{e}-01$ & 40.553 & 95.076 & 18.405 \\
set 7 & $7.396 \mathrm{e}-04$ & $4.554 \mathrm{e}-02$ & 125.614 & 65.652 & 80.895 \\
set 8 & $3.975 \mathrm{e}-04$ & $2.062 \mathrm{e}-01$ & 161.735 & 163.330 & 127.964 \\
set 9 & $4.088 \mathrm{e}-05$ & $3.344 \mathrm{e}-01$ & 14.808 & 118.521 & 23.686 \\
set 10 & $7.327 \mathrm{e}-04$ & $5.647 \mathrm{e}-01$ & 112.000 & 104.634 & 93.147 \\
set 11 & $2.841 \mathrm{e}-04$ & $1.503 \mathrm{e}-01$ & 60.778 & 142.216 & 58.064 \\
set 12 & $3.699 \mathrm{e}-04$ & $3.622 \mathrm{e}-01$ & 96.588 & 36.266 & 154.075 \\
set 13 & $2.937 \mathrm{e}-04$ & $7.459 \mathrm{e}-02$ & 171.093 & 127.724 & 75.773 \\
set 14 & $6.029 \mathrm{e}-05$ & $2.910 \mathrm{e}-01$ & 18.774 & 106.474 & 87.298 \\
set 15 & $7.092 \mathrm{e}-04$ & $2.583 \mathrm{e}-02$ & 98.649 & 85.551 & 86.981 \\
set 16 & $3.625 \mathrm{e}-04$ & $4.759 \mathrm{e}-01$ & 6.220 & 61.131 & 20.463 \\
set 17 & $5.491 \mathrm{e}-04$ & $6.128 \mathrm{e}-01$ & 32.972 & 150.627 & 27.701 \\
set 18 & $5.574 \mathrm{e}-04$ & $4.539 \mathrm{e}-01$ & 130.699 & 59.096 & 57.961 \\
set 19 & $7.986 \mathrm{e}-04$ & $1.237 \mathrm{e}-01$ & 178.354 & 24.726 & 2.195 \\
set 20 & $5.839 \mathrm{e}-04$ & $6.620 \mathrm{e}-01$ & 18.101 & 97.941 & 87.799
\end{tabular}

\section{Isopinocampheol run 1-B}

\section{Random start coordinates}

The the orientations when starting from random coordinates are summarized in the tables below. Note that the Euler angles might again be different due to completely different molecular frames as random coordinates are used. An additional change is observed when $(+)$-IPC is obtained instead of the reference structure $((-)$ IPC). These differences are addressed in table S-61 (section 2.2.4). 
Table S-50: Orientational data of IPC 1 obtained by TITANIA on the last optimization iteration for the runs 1-B11 starting from random coordinates.

\begin{tabular}{l|rcrrr} 
Set & \multicolumn{1}{|c}{$\boldsymbol{A}_{\mathrm{zz}}$} & \multicolumn{1}{c}{$\boldsymbol{\alpha} /{ }^{\circ}$} & \multicolumn{1}{c}{$\boldsymbol{\beta} /{ }^{\circ}$} & \multicolumn{1}{c}{$\boldsymbol{\gamma} /^{\circ}$} \\
\hline set 1 & $8.761 \mathrm{e}-04$ & $1.028 \mathrm{e}-01$ & 147.085 & 100.577 & 114.383 \\
set 2 & $1.070 \mathrm{e}-03$ & $3.061 \mathrm{e}-01$ & 87.256 & 105.065 & 94.030 \\
set 3 & $-1.620 \mathrm{e}-03$ & $3.932 \mathrm{e}-01$ & 104.900 & 91.068 & 62.665 \\
set 4 & $7.724 \mathrm{e}-04$ & $5.369 \mathrm{e}-01$ & 171.971 & 155.873 & 165.391 \\
set 5 & $-6.737 \mathrm{e}-04$ & $4.671 \mathrm{e}-01$ & 119.095 & 83.488 & 81.372 \\
set 6 & $-1.028 \mathrm{e}-03$ & $4.308 \mathrm{e}-01$ & 35.464 & 95.488 & 48.392
\end{tabular}

Table S-51: Orientational data of IPC 1 obtained by TITANIA on the last optimization iteration of run 1-B17 starting from random coordinates.

\begin{tabular}{l|rrrrr} 
Set & \multicolumn{1}{|c}{$\boldsymbol{A}_{\mathrm{zz}}$} & \multicolumn{1}{c}{$\boldsymbol{R}$} & \multicolumn{1}{c}{$\boldsymbol{\alpha} /{ }^{\circ}$} & \multicolumn{1}{c}{$\boldsymbol{\beta} /{ }^{\circ}$} & \multicolumn{1}{c}{$\boldsymbol{\gamma}{ }^{\circ}$} \\
\hline set 1 & $-7.681 \mathrm{e}-04$ & $4.511 \mathrm{e}-01$ & 52.180 & 79.659 & 137.478 \\
set 2 & $8.362 \mathrm{e}-04$ & $5.444 \mathrm{e}-01$ & 1.126 & 149.778 & 38.602 \\
set 3 & $-1.536 \mathrm{e}-03$ & $3.932 \mathrm{e}-01$ & 99.348 & 57.215 & 177.915 \\
set 4 & $6.410 \mathrm{e}-04$ & $6.529 \mathrm{e}-01$ & 99.700 & 37.107 & 8.100 \\
set 5 & $6.322 \mathrm{e}-04$ & $5.876 \mathrm{e}-01$ & 76.542 & 37.249 & 35.177 \\
set 6 & $-8.246 \mathrm{e}-04$ & $3.698 \mathrm{e}-01$ & 22.922 & 54.446 & 160.331
\end{tabular}

Table S-52: Orientational data of IPC 1 obtained by TITANIA on the last optimization iteration of run 1-B23 starting from random coordinates.

\begin{tabular}{l|rcrrr} 
Set & \multicolumn{1}{c}{$\boldsymbol{A}_{\mathrm{zz}}$} & $\boldsymbol{R}$ & \multicolumn{1}{c}{$\boldsymbol{\alpha} /{ }^{\circ}$} & \multicolumn{1}{c}{$\boldsymbol{\beta} /{ }^{\circ}$} & \multicolumn{1}{c}{$\boldsymbol{\gamma} /^{\circ}$} \\
\hline set 1 & $-7.930 \mathrm{e}-04$ & $3.734 \mathrm{e}-01$ & 5.657 & 67.226 & 174.047 \\
set 2 & $8.394 \mathrm{e}-04$ & $5.928 \mathrm{e}-01$ & 23.021 & 99.508 & 75.947 \\
set 3 & $1.412 \mathrm{e}-03$ & $6.666 \mathrm{e}-01$ & 73.765 & 38.931 & 65.043 \\
set 4 & $6.853 \mathrm{e}-04$ & $4.852 \mathrm{e}-01$ & 90.762 & 36.795 & 67.020 \\
set 5 & $7.149 \mathrm{e}-04$ & $3.957 \mathrm{e}-01$ & 104.323 & 38.666 & 48.798 \\
set 6 & $-7.124 \mathrm{e}-04$ & $1.450 \mathrm{e}-01$ & 7.410 & 114.197 & 32.245
\end{tabular}


Table S-53: Orientational data of IPC 1 obtained by TITANIA on the last optimization iteration of run 1-B31 starting from random coordinates.

\begin{tabular}{l|rrrrr} 
Set & \multicolumn{1}{|c}{$\boldsymbol{A}_{\mathrm{zz}}$} & \multicolumn{1}{c}{$\boldsymbol{\alpha}$} & \multicolumn{1}{c}{$\boldsymbol{\alpha} /{ }^{\circ}$} & \multicolumn{1}{c}{$\boldsymbol{\beta} /{ }^{\circ}$} & \multicolumn{1}{c}{$\boldsymbol{\gamma} /^{\circ}$} \\
\hline set 1 & $-7.388 \mathrm{e}-04$ & $4.551 \mathrm{e}-01$ & 173.016 & 104.800 & 66.725 \\
set 2 & $8.174 \mathrm{e}-04$ & $5.442 \mathrm{e}-01$ & 17.093 & 103.766 & 148.573 \\
set 3 & $-1.362 \mathrm{e}-03$ & $4.058 \mathrm{e}-01$ & 120.420 & 91.006 & 107.575 \\
set 4 & $5.493 \mathrm{e}-04$ & $6.586 \mathrm{e}-01$ & 9.564 & 35.637 & 26.572 \\
set 5 & $5.732 \mathrm{e}-04$ & $5.565 \mathrm{e}-01$ & 16.734 & 17.548 & 15.394 \\
set 6 & $-6.977 \mathrm{e}-04$ & $3.266 \mathrm{e}-01$ & 28.779 & 103.340 & 100.809
\end{tabular}

Table S-54: Orientational data of IPC 1 obtained by TITANIA on the last optimization iteration of run 1-B39 starting from random coordinates.

\begin{tabular}{l|rcrrr} 
Set & \multicolumn{1}{|c}{$\boldsymbol{A}_{\text {zz }}$} & \multicolumn{1}{c}{$\boldsymbol{c} \boldsymbol{\alpha} /{ }^{\circ}$} & \multicolumn{1}{c}{$\boldsymbol{\beta} /{ }^{\circ}$} & \multicolumn{1}{c}{$\boldsymbol{\gamma} /^{\circ}$} \\
\hline set 1 & $-7.563 \mathrm{e}-04$ & $4.713 \mathrm{e}-01$ & 37.021 & 109.846 & 128.910 \\
set 2 & $8.074 \mathrm{e}-04$ & $5.659 \mathrm{e}-01$ & 149.439 & 115.065 & 16.169 \\
set 3 & $-1.246 \mathrm{e}-03$ & $4.302 \mathrm{e}-01$ & 162.465 & 75.020 & 149.525 \\
set 4 & $5.184 \mathrm{e}-04$ & $6.173 \mathrm{e}-01$ & 33.891 & 74.574 & 60.898 \\
set 5 & $5.356 \mathrm{e}-04$ & $5.123 \mathrm{e}-01$ & 35.672 & 59.957 & 48.165 \\
set 6 & $-6.684 \mathrm{e}-04$ & $3.549 \mathrm{e}-01$ & 64.153 & 89.393 & 152.698
\end{tabular}

\section{Isopinocampheol run 1-C}

\section{Random start coordinates}

The the orientations when starting from random coordinates are summarized in the tables below. Note that the Euler angles might again be different due to completely different molecular frames as random coordinates are used. An additional change is observed when $(+)$-IPC is obtained instead of the reference structure $((-) \mathrm{IPC})$. These differences are addressed in table S-62 (section 2.2.4). 
Table S-55: Orientational data of IPC 1 obtained by TITANIA on the last optimization iteration for the runs $\mathbf{1 - C 1 1}$ starting from random coordinates.

\begin{tabular}{l|rrrrr} 
Set & \multicolumn{1}{|c}{$\boldsymbol{A}_{\mathrm{zz}}$} & \multicolumn{1}{c}{$\boldsymbol{R}$} & \multicolumn{1}{c}{$\boldsymbol{\alpha} /^{\circ}$} & \multicolumn{1}{c}{$\boldsymbol{\beta} /{ }^{\circ}$} & \multicolumn{1}{c}{$\boldsymbol{\gamma}{ }^{\circ}$} \\
\hline set 1 & $-8.138 \mathrm{e}-04$ & $5.915 \mathrm{e}-01$ & 154.862 & 100.130 & 157.192 \\
set 2 & $1.164 \mathrm{e}-03$ & $3.456 \mathrm{e}-01$ & 177.718 & 73.079 & 78.584 \\
set 7 & $-5.766 \mathrm{e}-04$ & $3.076 \mathrm{e}-01$ & 146.160 & 110.585 & 142.667 \\
set 8 & $1.286 \mathrm{e}-03$ & $3.201 \mathrm{e}-01$ & 2.008 & 75.336 & 79.152 \\
set 9 & $1.339 \mathrm{e}-03$ & $2.998 \mathrm{e}-01$ & 1.006 & 73.883 & 77.707 \\
set 10 & $1.133 \mathrm{e}-03$ & $3.579 \mathrm{e}-01$ & 177.227 & 72.971 & 78.373
\end{tabular}

Table S-56: Orientational data of IPC 1 obtained by TITANIA on the last optimization iteration of run 1-C17 starting from random coordinates.

\begin{tabular}{l|rcrrr} 
Set & \multicolumn{1}{|c}{$\boldsymbol{A}_{\mathrm{zz}}$} & \multicolumn{1}{c}{} & $\boldsymbol{\alpha} /{ }^{\circ}$ & \multicolumn{1}{c}{$\boldsymbol{\beta} /{ }^{\circ}$} & \multicolumn{1}{c}{$\boldsymbol{\gamma} /{ }^{\circ}$} \\
\hline set 1 & $-7.702 \mathrm{e}-04$ & $4.737 \mathrm{e}-01$ & 64.511 & 81.273 & 130.223 \\
set 2 & $9.101 \mathrm{e}-04$ & $5.175 \mathrm{e}-01$ & 36.811 & 140.863 & 71.257 \\
set 7 & $-6.302 \mathrm{e}-04$ & $6.271 \mathrm{e}-01$ & 58.117 & 88.763 & 116.314 \\
set 8 & $9.454 \mathrm{e}-04$ & $5.545 \mathrm{e}-01$ & 38.761 & 137.280 & 73.684 \\
set 9 & $9.951 \mathrm{e}-04$ & $5.617 \mathrm{e}-01$ & 43.329 & 139.714 & 73.651 \\
set 10 & $8.845 \mathrm{e}-04$ & $5.322 \mathrm{e}-01$ & 35.748 & 141.047 & 70.758
\end{tabular}

Table S-57: Orientational data of IPC 1 obtained by TITANIA on the last optimization iteration of run 1-C23 starting from random coordinates.

\begin{tabular}{l|rcrrr} 
Set & \multicolumn{1}{c}{$\boldsymbol{A}_{\mathrm{zz}}$} & $\boldsymbol{R}$ & $\boldsymbol{\alpha} /{ }^{\circ}$ & \multicolumn{1}{c}{$\boldsymbol{\beta} /{ }^{\circ}$} & \multicolumn{1}{c}{$\boldsymbol{\gamma} /^{\circ}$} \\
\hline set 1 & $-7.716 \mathrm{e}-04$ & $3.711 \mathrm{e}-01$ & 49.994 & 71.696 & 124.149 \\
set 2 & $8.494 \mathrm{e}-04$ & $5.940 \mathrm{e}-01$ & 43.828 & 134.097 & 71.188 \\
set 7 & $-6.154 \mathrm{e}-04$ & $5.683 \mathrm{e}-01$ & 45.892 & 78.545 & 112.278 \\
set 8 & $8.794 \mathrm{e}-04$ & $6.532 \mathrm{e}-01$ & 44.086 & 130.952 & 72.765 \\
set 9 & $-9.314 \mathrm{e}-04$ & $6.665 \mathrm{e}-01$ & 54.650 & 56.982 & 125.656 \\
set 10 & $8.252 \mathrm{e}-04$ & $6.120 \mathrm{e}-01$ & 42.917 & 134.094 & 70.534
\end{tabular}


Table S-58: Orientational data of IPC 1 obtained by TITANIA on the last optimization iteration of run 1-C31 starting from random coordinates.

\begin{tabular}{l|rcrrr} 
Set & \multicolumn{1}{c}{$\boldsymbol{A}_{\mathrm{zz}}$} & $\boldsymbol{R}$ & $\boldsymbol{\alpha} /{ }^{\circ}$ & \multicolumn{1}{c}{$\boldsymbol{\beta} /{ }^{\circ}$} & \multicolumn{1}{c}{$\boldsymbol{\gamma} /^{\circ}$} \\
\hline set 1 & $-7.127 \mathrm{e}-04$ & $4.347 \mathrm{e}-01$ & 66.768 & 93.187 & 144.325 \\
set 2 & $7.422 \mathrm{e}-04$ & $6.212 \mathrm{e}-01$ & 17.020 & 151.744 & 71.691 \\
set 7 & $-5.896 \mathrm{e}-04$ & $6.126 \mathrm{e}-01$ & 56.044 & 100.473 & 132.928 \\
set 8 & $-7.607 \mathrm{e}-04$ & $6.520 \mathrm{e}-01$ & 60.696 & 79.329 & 148.973 \\
set 9 & $-8.116 \mathrm{e}-04$ & $6.491 \mathrm{e}-01$ & 63.640 & 78.859 & 144.652 \\
set 10 & $7.235 \mathrm{e}-04$ & $6.365 \mathrm{e}-01$ & 15.911 & 151.761 & 70.907
\end{tabular}

Table S-59: Orientational data of IPC 1 obtained by TITANIA on the last optimization iteration of run 1-C39 starting from random coordinates.

\begin{tabular}{l|rcrrr} 
Set & \multicolumn{1}{|c}{$\boldsymbol{A}_{\mathrm{zz}}$} & \multicolumn{1}{c}{$\boldsymbol{c} \boldsymbol{\alpha} /{ }^{\circ}$} & \multicolumn{1}{c}{$\boldsymbol{\beta} /{ }^{\circ}$} & \multicolumn{1}{c}{$\boldsymbol{\gamma}{ }^{\circ}$} \\
\hline set 1 & $-7.491 \mathrm{e}-04$ & $4.611 \mathrm{e}-01$ & 57.038 & 105.795 & 168.339 \\
set 2 & $8.255 \mathrm{e}-04$ & $5.360 \mathrm{e}-01$ & 163.157 & 153.147 & 71.950 \\
set 7 & $-6.307 \mathrm{e}-04$ & $6.278 \mathrm{e}-01$ & 66.413 & 119.704 & 169.448 \\
set 8 & $8.396 \mathrm{e}-04$ & $5.873 \mathrm{e}-01$ & 170.099 & 155.721 & 78.907 \\
set 9 & $8.940 \mathrm{e}-04$ & $5.913 \mathrm{e}-01$ & 163.086 & 153.570 & 76.169 \\
set 10 & $8.035 \mathrm{e}-04$ & $5.522 \mathrm{e}-01$ & 162.967 & 153.085 & 71.148
\end{tabular}




\subsubsection{Change in Orientation}

\section{1-A}

Table S-60: Generalized angle $\beta$ enclosed by the reference structure of IPC and the structure obtained by TITANIA from the last optimization step of the runs 1-A using random start coordinates. The data were obtained by transformation of the respective structures to a common reference frame, followed by recalculating the alignment tensors with the in-house software RDC@hotFCHT. ${ }^{[9]}$

\begin{tabular}{l|rrrrr} 
& \multicolumn{5}{|c}{$\boldsymbol{\beta} /{ }^{\circ}$} \\
Set & $\mathbf{1 - A 1 1}$ & $\mathbf{1 - A 1 7}$ & $\mathbf{1 - A} 23$ & $\mathbf{1 - A 3 1}$ & $\mathbf{1 - A 3 9}$ \\
\hline set 1 & 54.278 & 3.333 & 2.103 & 8.992 & 2.518 \\
set 2 & 33.157 & 7.799 & 3.040 & 5.246 & 0.623 \\
set 3 & 44.832 & 5.824 & 7.441 & 7.603 & 1.603 \\
set 4 & 67.560 & 3.752 & 5.225 & 3.246 & 2.627 \\
set 5 & 114.715 & 6.124 & 2.802 & 4.747 & 2.401 \\
set 6 & 17.816 & 9.912 & 5.349 & 7.789 & 0.994 \\
set 7 & 39.553 & 11.291 & 6.148 & 6.288 & 2.845 \\
set 8 & 15.250 & 7.295 & 4.019 & 3.667 & 2.447 \\
set 9 & 12.462 & 9.949 & 4.959 & 6.350 & 2.163 \\
set 10 & 54.355 & 2.660 & 4.665 & 8.359 & 1.921 \\
set 11 & 46.911 & 8.392 & 4.098 & 4.104 & 2.232 \\
set 12 & 48.198 & 6.275 & 3.873 & 2.715 & 2.152 \\
set 13 & 84.427 & 4.763 & 2.814 & 6.104 & 1.421 \\
set 14 & 107.470 & 4.669 & 3.671 & 7.301 & 3.134 \\
set 15 & 72.467 & 6.875 & 2.946 & 7.378 & 3.417 \\
set 16 & 18.298 & 6.513 & 6.743 & 10.020 & 1.128 \\
set 17 & 21.621 & 8.970 & 4.400 & 4.014 & 3.573 \\
set 18 & 26.787 & 7.159 & 5.825 & 7.009 & 2.015 \\
set 19 & 16.181 & 7.160 & 5.214 & 6.231 & 1.532 \\
set 20 & 97.894 & 6.811 & 3.671 & 6.642 & 3.426
\end{tabular}

The $\beta$ angles of 1-A17 to 1-A39 enclosed by the alignment tensors obtained from the two structures (TITANIA optimized and reference) in a common frame are comparable to those reported in table S-35 (C3 epimer starting structure). The small differences are due to structural changes elicited by experimental errors and the change of holonomic terms. The standard approach is to extract them from the input structure. This is not possible for random coordinates. 


\section{1-B}

Table S-61: Generalized angle $\beta$ enclosed by the reference structure of IPC and the structure obtained by TITANIA from the last optimization step of the runs 1-B using random start coordinates. The data were obtained by transformation of the respective structures to a common reference frame, followed by recalculating the alignment tensors with the in-house software RDC@hotFCHT. ${ }^{[9]}$

\begin{tabular}{l|rrrrr} 
& \multicolumn{5}{|c}{$\boldsymbol{\beta}{ }^{\circ}$} \\
Set & 1-B11 & 1-B17 & 1-B23 & 1-B31 & 1-B39 \\
\hline set 1 & 81.835 & 5.163 & 15.189 & 5.888 & 7.866 \\
set 2 & 73.678 & 6.842 & 20.081 & 5.335 & 7.010 \\
set 3 & 76.385 & 4.555 & 27.076 & 2.144 & 6.420 \\
set 4 & 82.391 & 3.093 & 37.250 & 2.605 & 4.194 \\
set 5 & 94.694 & 4.626 & 32.549 & 1.936 & 4.072 \\
set 6 & 48.408 & 5.425 & 20.847 & 3.985 & 9.193
\end{tabular}

As in 1-A11 the example 1-B11 shows large differences (large $\beta$ angles) when using 11 RDCs. In addition 1-B23 shows large $\beta$ angles since the inverse $\mathrm{C} 2-\mathrm{C} 10 \mathrm{RDC}$ vector is found for the final structure. The $\beta$ angles of all other runs originate from the use of different holonomic terms (see above).

\section{$1-\mathrm{C}$}

Table S-62: Generalized angle $\beta$ enclosed by the reference structure of IPC and the structure obtained by TITANIA from the last optimization step of the runs 1-C using random start coordinates. The data were obtained by transformation of the respective structures to a common reference frame, followed by recalculating the alignment tensors with the in-house software RDC@hotFCHT. ${ }^{[9]}$

\begin{tabular}{l|rrrrr} 
& \multicolumn{5}{|c}{$\boldsymbol{\beta} /{ }^{\circ}$} \\
Set & 1-C11 & 1-C17 & 1-C23 & 1-C31 & 1-C39 \\
\hline set 1 & 46.844 & 8.346 & 14.694 & 3.082 & 8.199 \\
set 2 & 84.687 & 7.423 & 15.061 & 3.329 & 8.165 \\
set 7 & 41.721 & 6.795 & 10.885 & 3.281 & 8.517 \\
set 8 & 84.353 & 7.065 & 12.458 & 3.510 & 8.208 \\
set 9 & 86.778 & 6.977 & 20.640 & 3.813 & 8.081 \\
set 10 & 83.159 & 7.470 & 14.322 & 3.213 & 8.183
\end{tabular}


As before run 1-C11 shows large differences (large $\beta$ angles) when using 11 RDCs. In addition 1-C23 shows large $\beta$ angles, for the same reason as in 1-B23. The inverse $\mathrm{C} 2-\mathrm{C} 10 \mathrm{RDC}$ vector is found for the final structure. The $\beta$ angles of all other runs originate from the use of different holonomic terms (see above). 


\subsubsection{Optimization Trajectories}
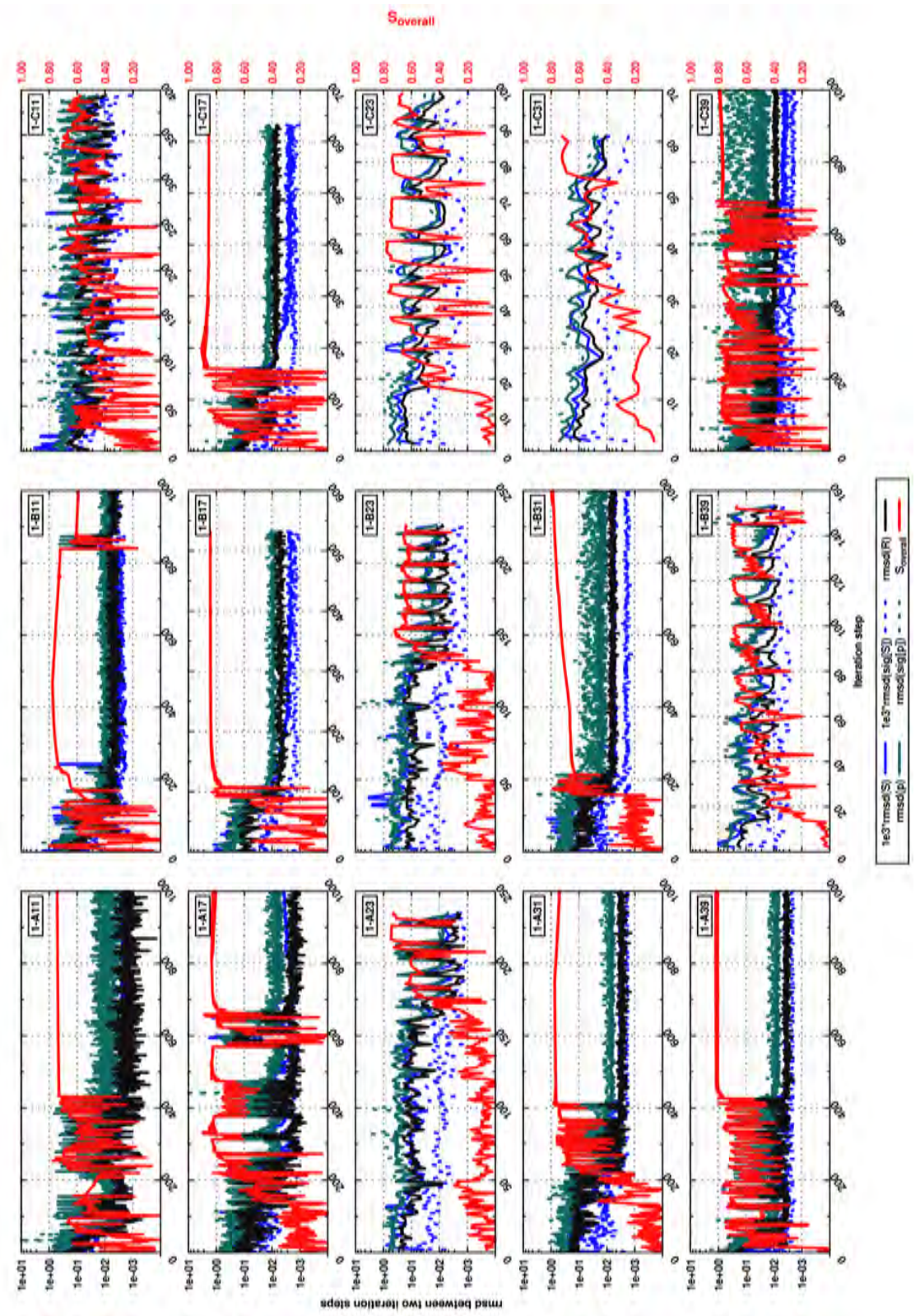

Figure S-14: Trajectory of the Monte-Carlo $\mathrm{rmsds}$ obtained for the individual runs of the setups $\mathbf{1 - A}, \mathbf{B}$ and $\mathbf{C}$. Shown are the data obtained by the default redundant internal coordinates algorithm starting from random coordinates. 

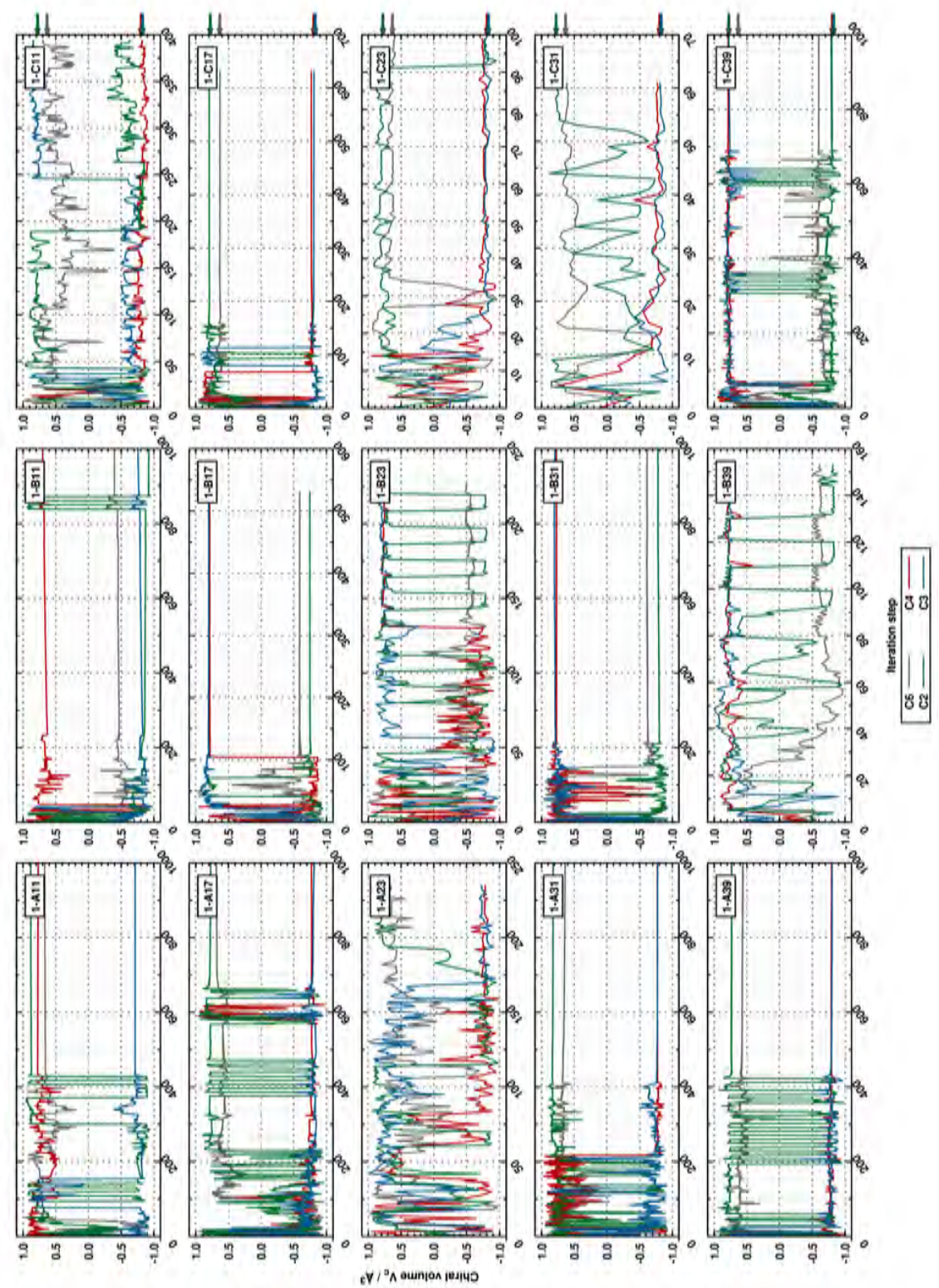

Figure S-15: Trajectory of the normalized chiral volumes obtained for the individual runs of the setups $\mathbf{1 - A}, \mathbf{B}$ and $\mathbf{C}$. Shown are the data obtained by the default redundant internal coordinates algorithm starting from random coordinates. The arrows at the top indicate the values of the reference structure (correct configuration). Note that in contrast to the runs starting from the C3 epimer also the enantiomeric solution is obtained (1-B17, 31, 39 and 1-C39). 
As before none of the runs with 11 RDCs converges to the correct relative configuration in all cases. This behavior is discussed in the main text. The two runs 1-B23 and 1-B39 do not show a stable configuration on C2 and the final structure has the wrong configuration at this center. 1-B23 can easily be recognized as inverse vector solution (see fig. S-16).

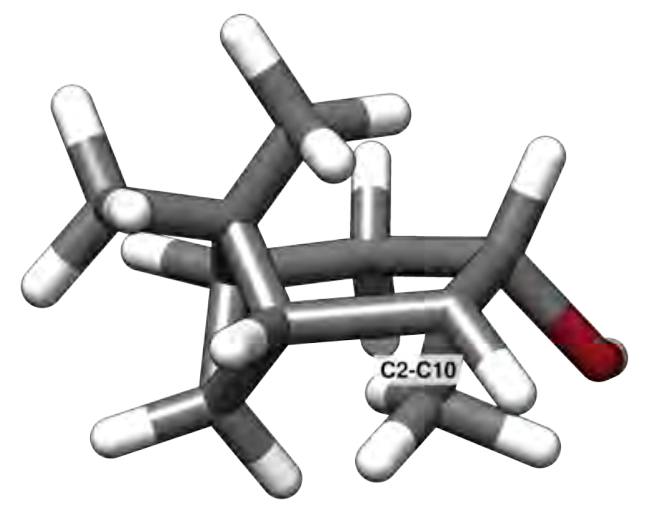

Figure S-16: Final 3D structure for the run 1-B23. The $\mathrm{C} 2-\mathrm{C} 10$ bond is represented by the inverse RDC solution. This can be identified with some basic chemistry knowledge.

The wrong configuration of 1-B39 can be identified by the very short C2$\mathrm{C} 10$ bond $(1.13 \AA)$. Both structures were rerun while forcing 1000 iteration steps, ending in a stable trajectory with the correct relative configuration.

\subsection{Vector addition algorithm}

\subsubsection{Output orientations}

\section{Isopinocampheol run 1-D}

\section{Structure generation via vector addition}

Additionally to the redundant internal coordinates algorithm the optimization was performed using the non-default vector addition algorithm. The orientations obtained are summarized in the tables below. Changes of the Euler angles compared to the literature data listed above are due to the change of the initial reference frames (TITANIA used the principle axis system of the molecule) and the added errors. These differences are addressed in table S-78 (section 2.4.2). 
Table S-63: Orientational data of IPC 1 obtained by TITANIA on the last optimization iteration for the run 1-D11 when using the non-default vector addition algorithm.

\begin{tabular}{l|rcrrr} 
Set & \multicolumn{1}{c}{$\boldsymbol{A}_{\mathrm{zz}}$} & $\boldsymbol{R}$ & \multicolumn{1}{c}{$\boldsymbol{\alpha} /{ }^{\circ}$} & \multicolumn{1}{c}{$\boldsymbol{\beta} /{ }^{\circ}$} & \multicolumn{1}{c}{$\boldsymbol{\gamma}{ }^{\circ}$} \\
\hline set 1 & $5.373 \mathrm{e}-04$ & $2.042 \mathrm{e}-01$ & 174.491 & 39.947 & 75.871 \\
set 2 & $1.038 \mathrm{e}-04$ & $2.681 \mathrm{e}-01$ & 133.318 & 75.386 & 133.293 \\
set 3 & $-1.221 \mathrm{e}-04$ & $3.855 \mathrm{e}-01$ & 23.708 & 87.733 & 109.974 \\
set 4 & $-5.633 \mathrm{e}-04$ & $1.638 \mathrm{e}-01$ & 105.955 & 44.595 & 30.299 \\
set 5 & $2.840 \mathrm{e}-04$ & $6.189 \mathrm{e}-01$ & 144.284 & 97.006 & 97.110 \\
set 6 & $9.636 \mathrm{e}-04$ & $4.520 \mathrm{e}-01$ & 156.527 & 143.191 & 152.451 \\
set 7 & $9.946 \mathrm{e}-04$ & $1.361 \mathrm{e}-01$ & 39.369 & 62.490 & 52.623 \\
set 8 & $-4.378 \mathrm{e}-04$ & $6.335 \mathrm{e}-01$ & 54.994 & 42.280 & 33.234 \\
set 9 & $-8.343 \mathrm{e}-05$ & $6.136 \mathrm{e}-01$ & 142.655 & 138.007 & 16.961 \\
set 10 & $7.712 \mathrm{e}-04$ & $1.712 \mathrm{e}-01$ & 134.452 & 13.446 & 108.690 \\
set 11 & $3.913 \mathrm{e}-04$ & $3.432 \mathrm{e}-01$ & 81.937 & 150.351 & 32.083 \\
set 12 & $4.075 \mathrm{e}-04$ & $4.888 \mathrm{e}-01$ & 152.925 & 119.223 & 73.971 \\
set 13 & $3.653 \mathrm{e}-04$ & $1.550 \mathrm{e}-01$ & 27.367 & 155.243 & 0.254 \\
set 14 & $9.404 \mathrm{e}-05$ & $2.873 \mathrm{e}-01$ & 36.080 & 27.308 & 174.843 \\
set 15 & $8.313 \mathrm{e}-04$ & $1.613 \mathrm{e}-01$ & 174.945 & 48.744 & 55.599 \\
set 16 & $4.463 \mathrm{e}-04$ & $1.898 \mathrm{e}-01$ & 112.773 & 116.491 & 170.623 \\
set 17 & $-8.387 \mathrm{e}-04$ & $4.194 \mathrm{e}-01$ & 92.152 & 54.816 & 43.156 \\
set 18 & $7.629 \mathrm{e}-04$ & $4.672 \mathrm{e}-01$ & 45.210 & 62.128 & 36.137 \\
set 19 & $7.171 \mathrm{e}-04$ & $5.343 \mathrm{e}-01$ & 105.610 & 77.381 & 169.026 \\
set 20 & $-5.477 \mathrm{e}-04$ & $4.438 \mathrm{e}-01$ & 110.761 & 121.855 & 117.043
\end{tabular}


Table S-64: Orientational data of IPC 1 obtained by TITANIA on the last optimization iteration of run 1-D17 when using the non-default vector addition algorithm.

\begin{tabular}{l|rcrrr} 
Set & \multicolumn{1}{c}{$\boldsymbol{A}_{\mathrm{zz}}$} & $\boldsymbol{R}$ & \multicolumn{1}{c}{$\boldsymbol{\alpha} /{ }^{\circ}$} & \multicolumn{1}{c}{$\boldsymbol{\beta} /{ }^{\circ}$} & \multicolumn{1}{c}{$\boldsymbol{\gamma} /{ }^{\circ}$} \\
\hline set 1 & $5.419 \mathrm{e}-04$ & $2.683 \mathrm{e}-01$ & 9.021 & 36.607 & 90.171 \\
set 2 & $9.144 \mathrm{e}-05$ & $4.008 \mathrm{e}-01$ & 138.048 & 80.914 & 136.202 \\
set 3 & $-1.167 \mathrm{e}-04$ & $5.925 \mathrm{e}-01$ & 158.072 & 92.805 & 114.997 \\
set 4 & $4.143 \mathrm{e}-04$ & $4.151 \mathrm{e}-01$ & 88.219 & 128.259 & 45.551 \\
set 5 & $2.706 \mathrm{e}-04$ & $3.798 \mathrm{e}-01$ & 145.308 & 106.148 & 89.560 \\
set 6 & $8.609 \mathrm{e}-04$ & $5.053 \mathrm{e}-01$ & 170.674 & 137.355 & 146.594 \\
set 7 & $7.984 \mathrm{e}-04$ & $1.426 \mathrm{e}-01$ & 28.327 & 57.799 & 56.824 \\
set 8 & $4.434 \mathrm{e}-04$ & $1.455 \mathrm{e}-01$ & 80.734 & 122.264 & 12.327 \\
set 9 & $7.443 \mathrm{e}-05$ & $2.305 \mathrm{e}-01$ & 124.121 & 150.843 & 150.070 \\
set 10 & $7.200 \mathrm{e}-04$ & $4.211 \mathrm{e}-01$ & 10.869 & 24.973 & 105.082 \\
set 11 & $3.641 \mathrm{e}-04$ & $2.843 \mathrm{e}-01$ & 25.436 & 155.844 & 36.503 \\
set 12 & $4.131 \mathrm{e}-04$ & $5.149 \mathrm{e}-01$ & 8.788 & 116.895 & 70.597 \\
set 13 & $3.238 \mathrm{e}-04$ & $2.873 \mathrm{e}-01$ & 17.705 & 20.404 & 174.540 \\
set 14 & $7.652 \mathrm{e}-05$ & $5.849 \mathrm{e}-01$ & 85.755 & 12.407 & 109.289 \\
set 15 & $7.089 \mathrm{e}-04$ & $3.531 \mathrm{e}-02$ & 5.030 & 41.847 & 71.173 \\
set 16 & $3.885 \mathrm{e}-04$ & $3.207 \mathrm{e}-01$ & 150.003 & 117.563 & 173.687 \\
set 17 & $6.435 \mathrm{e}-04$ & $5.210 \mathrm{e}-01$ & 77.645 & 142.149 & 51.667 \\
set 18 & $6.205 \mathrm{e}-04$ & $4.784 \mathrm{e}-01$ & 45.213 & 57.659 & 36.705 \\
set 19 & $7.955 \mathrm{e}-04$ & $1.423 \mathrm{e}-01$ & 70.305 & 101.398 & 10.529 \\
set 20 & $-5.876 \mathrm{e}-04$ & $4.930 \mathrm{e}-01$ & 104.722 & 121.034 & 114.784
\end{tabular}


Table S-65: Orientational data of IPC 1 obtained by TITANIA on the last optimization iteration of run 1-D23 when using the non-default vector addition algorithm.

\begin{tabular}{l|rcrrr} 
Set & \multicolumn{1}{c}{$\boldsymbol{A}_{\mathrm{zz}}$} & $\boldsymbol{R}$ & \multicolumn{1}{c}{$\boldsymbol{\alpha} /{ }^{\circ}$} & \multicolumn{1}{c}{$\boldsymbol{\beta} /{ }^{\circ}$} & \multicolumn{1}{c}{$\boldsymbol{\gamma}{ }^{\circ}$} \\
\hline set 1 & $5.422 \mathrm{e}-04$ & $2.953 \mathrm{e}-01$ & 6.547 & 40.663 & 93.785 \\
set 2 & $7.777 \mathrm{e}-05$ & $3.952 \mathrm{e}-01$ & 137.781 & 81.261 & 141.378 \\
set 3 & $1.192 \mathrm{e}-04$ & $5.651 \mathrm{e}-01$ & 2.096 & 70.688 & 22.769 \\
set 4 & $4.288 \mathrm{e}-04$ & $3.641 \mathrm{e}-01$ & 84.188 & 125.257 & 40.916 \\
set 5 & $2.845 \mathrm{e}-04$ & $3.795 \mathrm{e}-01$ & 137.694 & 109.125 & 82.773 \\
set 6 & $8.685 \mathrm{e}-04$ & $4.910 \mathrm{e}-01$ & 166.888 & 140.944 & 138.662 \\
set 7 & $7.966 \mathrm{e}-04$ & $1.002 \mathrm{e}-01$ & 14.489 & 56.143 & 52.502 \\
set 8 & $4.357 \mathrm{e}-04$ & $1.245 \mathrm{e}-01$ & 90.698 & 117.437 & 9.536 \\
set 9 & $7.061 \mathrm{e}-05$ & $3.380 \mathrm{e}-01$ & 94.943 & 143.508 & 128.948 \\
set 10 & $7.164 \mathrm{e}-04$ & $5.193 \mathrm{e}-01$ & 2.455 & 30.800 & 109.095 \\
set 11 & $3.439 \mathrm{e}-04$ & $2.539 \mathrm{e}-01$ & 17.640 & 153.142 & 29.169 \\
set 12 & $4.369 \mathrm{e}-04$ & $4.309 \mathrm{e}-01$ & 7.727 & 116.793 & 65.726 \\
set 13 & $3.089 \mathrm{e}-04$ & $1.993 \mathrm{e}-01$ & 22.045 & 22.709 & 163.635 \\
set 14 & $-8.449 \mathrm{e}-05$ & $4.659 \mathrm{e}-01$ & 86.259 & 104.079 & 109.945 \\
set 15 & $7.079 \mathrm{e}-04$ & $2.872 \mathrm{e}-02$ & 97.047 & 41.276 & 71.833 \\
set 16 & $3.968 \mathrm{e}-04$ & $3.593 \mathrm{e}-01$ & 143.684 & 120.315 & 170.488 \\
set 17 & $6.474 \mathrm{e}-04$ & $5.077 \mathrm{e}-01$ & 74.058 & 139.950 & 47.386 \\
set 18 & $6.324 \mathrm{e}-04$ & $4.819 \mathrm{e}-01$ & 41.151 & 56.150 & 31.643 \\
set 19 & $8.226 \mathrm{e}-04$ & $7.408 \mathrm{e}-02$ & 71.928 & 96.851 & 8.674 \\
set 20 & $-6.087 \mathrm{e}-04$ & $5.421 \mathrm{e}-01$ & 100.258 & 124.743 & 106.130
\end{tabular}


Table S-66: Orientational data of IPC 1 obtained by TITANIA on the last optimization iteration of run 1-D31 when using the non-default vector addition algorithm.

\begin{tabular}{l|rcrrr} 
Set & \multicolumn{1}{c}{$\boldsymbol{A}_{\mathrm{zz}}$} & $\boldsymbol{R}$ & \multicolumn{1}{c}{$\boldsymbol{\alpha} /{ }^{\circ}$} & \multicolumn{1}{c}{$\boldsymbol{\beta} /{ }^{\circ}$} & \multicolumn{1}{c}{$\boldsymbol{\gamma} /^{\circ}$} \\
\hline set 1 & $5.808 \mathrm{e}-04$ & $3.500 \mathrm{e}-01$ & 152.618 & 36.719 & 129.517 \\
set 2 & $8.500 \mathrm{e}-05$ & $3.771 \mathrm{e}-01$ & 84.661 & 81.637 & 3.749 \\
set 3 & $-8.143 \mathrm{e}-05$ & $3.999 \mathrm{e}-01$ & 150.775 & 44.056 & 152.961 \\
set 4 & $3.267 \mathrm{e}-04$ & $5.536 \mathrm{e}-01$ & 91.801 & 116.906 & 80.228 \\
set 5 & $2.282 \mathrm{e}-04$ & $2.315 \mathrm{e}-01$ & 105.807 & 108.839 & 105.951 \\
set 6 & $8.329 \mathrm{e}-04$ & $5.664 \mathrm{e}-01$ & 144.445 & 149.298 & 164.489 \\
set 7 & $6.220 \mathrm{e}-04$ & $5.170 \mathrm{e}-02$ & 162.979 & 54.598 & 105.010 \\
set 8 & $2.573 \mathrm{e}-04$ & $4.079 \mathrm{e}-01$ & 100.349 & 106.334 & 41.365 \\
set 9 & $6.041 \mathrm{e}-05$ & $1.480 \mathrm{e}-01$ & 100.168 & 176.397 & 45.151 \\
set 10 & $6.206 \mathrm{e}-04$ & $4.546 \mathrm{e}-01$ & 150.536 & 21.461 & 145.007 \\
set 11 & $2.886 \mathrm{e}-04$ & $2.668 \mathrm{e}-01$ & 137.185 & 147.350 & 63.502 \\
set 12 & $4.487 \mathrm{e}-04$ & $3.509 \mathrm{e}-01$ & 163.675 & 113.105 & 97.211 \\
set 13 & $2.899 \mathrm{e}-04$ & $3.908 \mathrm{e}-01$ & 109.262 & 156.869 & 13.863 \\
set 14 & $-7.818 \mathrm{e}-05$ & $4.865 \mathrm{e}-01$ & 99.147 & 112.849 & 156.148 \\
set 15 & $7.136 \mathrm{e}-04$ & $1.681 \mathrm{e}-01$ & 160.598 & 41.065 & 117.970 \\
set 16 & $3.379 \mathrm{e}-04$ & $3.937 \mathrm{e}-01$ & 14.660 & 58.488 & 14.345 \\
set 17 & $5.650 \mathrm{e}-04$ & $4.201 \mathrm{e}-01$ & 69.620 & 131.382 & 84.382 \\
set 18 & $-3.220 \mathrm{e}-04$ & $3.770 \mathrm{e}-01$ & 52.916 & 127.830 & 17.946 \\
set 19 & $7.162 \mathrm{e}-04$ & $2.887 \mathrm{e}-01$ & 90.691 & 88.466 & 25.740 \\
set 20 & $5.993 \mathrm{e}-04$ & $5.382 \mathrm{e}-01$ & 98.260 & 39.378 & 132.572
\end{tabular}


Table S-67: Orientational data of IPC 1 obtained by TITANIA on the last optimization iteration of run 1-D39 when using the non-default vector addition algorithm.

\begin{tabular}{l|rcrrr} 
Set & \multicolumn{1}{c}{$\boldsymbol{A}_{\mathrm{zz}}$} & $\boldsymbol{R}$ & \multicolumn{1}{c}{$\boldsymbol{\alpha} /{ }^{\circ}$} & \multicolumn{1}{c}{$\boldsymbol{\beta} /{ }^{\circ}$} & \multicolumn{1}{c}{$\boldsymbol{\gamma} /{ }^{\circ}$} \\
\hline set 1 & $6.537 \mathrm{e}-04$ & $1.980 \mathrm{e}-01$ & 176.084 & 57.511 & 103.435 \\
set 2 & $1.168 \mathrm{e}-04$ & $1.209 \mathrm{e}-01$ & 131.923 & 115.122 & 128.867 \\
set 3 & $1.329 \mathrm{e}-04$ & $6.661 \mathrm{e}-01$ & 21.177 & 91.627 & 20.967 \\
set 4 & $4.322 \mathrm{e}-04$ & $5.670 \mathrm{e}-01$ & 64.569 & 128.320 & 28.137 \\
set 5 & $2.641 \mathrm{e}-04$ & $3.147 \mathrm{e}-01$ & 110.795 & 125.524 & 70.914 \\
set 6 & $9.346 \mathrm{e}-04$ & $4.090 \mathrm{e}-01$ & 171.640 & 154.657 & 153.294 \\
set 7 & $8.031 \mathrm{e}-04$ & $5.941 \mathrm{e}-02$ & 176.832 & 66.736 & 65.927 \\
set 8 & $4.661 \mathrm{e}-04$ & $2.270 \mathrm{e}-01$ & 91.020 & 113.544 & 1.088 \\
set 9 & $-8.924 \mathrm{e}-05$ & $2.382 \mathrm{e}-01$ & 59.742 & 67.508 & 47.813 \\
set 10 & $6.985 \mathrm{e}-04$ & $5.202 \mathrm{e}-01$ & 6.170 & 47.530 & 108.602 \\
set 11 & $3.230 \mathrm{e}-04$ & $1.719 \mathrm{e}-01$ & 164.057 & 34.955 & 178.525 \\
set 12 & $4.940 \mathrm{e}-04$ & $1.481 \mathrm{e}-01$ & 149.735 & 131.613 & 61.722 \\
set 13 & $3.401 \mathrm{e}-04$ & $2.454 \mathrm{e}-01$ & 64.761 & 33.170 & 129.886 \\
set 14 & $8.389 \mathrm{e}-05$ & $4.301 \mathrm{e}-01$ & 114.177 & 37.535 & 90.348 \\
set 15 & $8.199 \mathrm{e}-04$ & $1.158 \mathrm{e}-01$ & 97.962 & 57.061 & 86.443 \\
set 16 & $3.179 \mathrm{e}-04$ & $6.111 \mathrm{e}-01$ & 164.189 & 117.542 & 175.132 \\
set 17 & $6.448 \mathrm{e}-04$ & $5.742 \mathrm{e}-01$ & 62.558 & 148.610 & 46.049 \\
set 18 & $-5.197 \mathrm{e}-04$ & $6.493 \mathrm{e}-01$ & 155.032 & 65.944 & 142.232 \\
set 19 & $9.158 \mathrm{e}-04$ & $1.450 \mathrm{e}-01$ & 61.396 & 96.706 & 5.354 \\
set 20 & $-7.089 \mathrm{e}-04$ & $6.287 \mathrm{e}-01$ & 96.120 & 141.355 & 105.311
\end{tabular}

\section{Isopinocampheol run 1-E}

\section{Structure generation via vector addition}

Additionally to the redundant internal coordinates algorithm the optimization was performed using the non-default vector addition algorithm. The orientations obtained are summarized in the tables below. Changes of the Euler angles compared to the literature data listed above are due to the change of the initial reference frames (TITANIA used the principle axis system of the molecule) and the added errors. These differences are addressed in table S-79 (section 2.4.2). 
Table S-68: Orientational data of IPC 1 obtained by TITANIA on the last optimization iteration for the run 1-E11.

\begin{tabular}{l|rrrrr} 
Set & \multicolumn{1}{|c}{$\boldsymbol{A}_{\mathrm{zz}}$} & \multicolumn{1}{c}{$\boldsymbol{R}$} & \multicolumn{1}{c}{$\boldsymbol{\alpha} /{ }^{\circ}$} & \multicolumn{1}{c}{$\boldsymbol{\beta} /{ }^{\circ}$} & \multicolumn{1}{c}{$\boldsymbol{\gamma}{ }^{\circ}$} \\
\hline set 1 & $-8.045 \mathrm{e}-04$ & $4.224 \mathrm{e}-01$ & 96.654 & 45.307 & 141.473 \\
set 2 & $9.842 \mathrm{e}-04$ & $3.760 \mathrm{e}-01$ & 102.637 & 119.671 & 163.307 \\
set 3 & $-1.559 \mathrm{e}-03$ & $4.380 \mathrm{e}-01$ & 0.144 & 86.486 & 141.153 \\
set 4 & $6.483 \mathrm{e}-04$ & $5.398 \mathrm{e}-01$ & 164.882 & 72.865 & 55.053 \\
set 5 & $7.506 \mathrm{e}-04$ & $5.074 \mathrm{e}-01$ & 165.107 & 94.059 & 51.050 \\
set 6 & $-8.986 \mathrm{e}-04$ & $3.824 \mathrm{e}-01$ & 60.266 & 78.975 & 150.058
\end{tabular}

Table S-69: Orientational data of IPC 1 obtained by TITANIA on the last optimization iteration of run 1-E17.

\begin{tabular}{l|rrrrr} 
Set & \multicolumn{1}{|c}{$\boldsymbol{A}_{\mathrm{zz}}$} & \multicolumn{1}{c}{$\boldsymbol{R}$} & \multicolumn{1}{c}{$\boldsymbol{\alpha}{ }^{\circ}$} & \multicolumn{1}{c}{$\boldsymbol{\beta} /{ }^{\circ}$} & \multicolumn{1}{c}{$\boldsymbol{\gamma} /^{\circ}$} \\
\hline set 1 & $-7.506 \mathrm{e}-04$ & $4.306 \mathrm{e}-01$ & 95.625 & 44.765 & 138.420 \\
set 2 & $9.079 \mathrm{e}-04$ & $4.336 \mathrm{e}-01$ & 101.463 & 118.080 & 164.331 \\
set 3 & $-1.433 \mathrm{e}-03$ & $3.668 \mathrm{e}-01$ & 172.173 & 83.773 & 137.762 \\
set 4 & $6.241 \mathrm{e}-04$ & $4.948 \mathrm{e}-01$ & 164.533 & 65.655 & 53.280 \\
set 5 & $6.060 \mathrm{e}-04$ & $5.223 \mathrm{e}-01$ & 167.754 & 84.704 & 48.102 \\
set 6 & $-8.748 \mathrm{e}-04$ & $5.243 \mathrm{e}-01$ & 59.604 & 76.589 & 145.749
\end{tabular}

Table S-70: Orientational data of IPC 1 obtained by TITANIA on the last optimization iteration of run 1-E23.

\begin{tabular}{l|rrrrr} 
Set & \multicolumn{1}{|c}{$\boldsymbol{A}_{\mathrm{zz}}$} & \multicolumn{1}{c}{$\boldsymbol{R}$} & \multicolumn{1}{c}{$\boldsymbol{\alpha} /{ }^{\circ}$} & \multicolumn{1}{c}{$\boldsymbol{\beta} /{ }^{\circ}$} & \multicolumn{1}{c}{$\boldsymbol{\gamma}{ }^{\circ}$} \\
\hline set 1 & $-7.473 \mathrm{e}-04$ & $4.975 \mathrm{e}-01$ & 100.985 & 42.990 & 139.459 \\
set 2 & $9.475 \mathrm{e}-04$ & $4.088 \mathrm{e}-01$ & 97.794 & 116.732 & 163.104 \\
set 3 & $-1.476 \mathrm{e}-03$ & $3.611 \mathrm{e}-01$ & 168.306 & 85.335 & 138.283 \\
set 4 & $6.407 \mathrm{e}-04$ & $5.536 \mathrm{e}-01$ & 166.678 & 65.822 & 51.849 \\
set 5 & $6.173 \mathrm{e}-04$ & $5.787 \mathrm{e}-01$ & 169.087 & 83.561 & 46.772 \\
set 6 & $-8.716 \mathrm{e}-04$ & $4.251 \mathrm{e}-01$ & 62.549 & 78.948 & 149.451
\end{tabular}


Table S-71: Orientational data of IPC 1 obtained by TITANIA on the last optimization iteration of run 1-E31.

\begin{tabular}{l|rrrrr} 
Set & \multicolumn{1}{|c}{$\boldsymbol{A}_{\mathrm{zz}}$} & \multicolumn{1}{c}{$\boldsymbol{\alpha}$} & \multicolumn{1}{c}{$\boldsymbol{\alpha} /{ }^{\circ}$} & \multicolumn{1}{c}{$\boldsymbol{\beta} /{ }^{\circ}$} & \multicolumn{1}{c}{$\boldsymbol{\gamma}{ }^{\circ}$} \\
\hline set 1 & $-7.730 \mathrm{e}-04$ & $5.076 \mathrm{e}-01$ & 105.411 & 46.667 & 138.334 \\
set 2 & $9.805 \mathrm{e}-04$ & $3.926 \mathrm{e}-01$ & 104.582 & 120.649 & 167.947 \\
set 3 & $-1.415 \mathrm{e}-03$ & $3.527 \mathrm{e}-01$ & 171.479 & 90.805 & 139.949 \\
set 4 & $-5.611 \mathrm{e}-04$ & $6.561 \mathrm{e}-01$ & 160.802 & 109.762 & 139.541 \\
set 5 & $5.613 \mathrm{e}-04$ & $6.531 \mathrm{e}-01$ & 159.936 & 90.942 & 46.574 \\
set 6 & $-8.536 \mathrm{e}-04$ & $3.913 \mathrm{e}-01$ & 69.350 & 81.978 & 148.908
\end{tabular}

Table S-72: Orientational data of IPC 1 obtained by TITANIA on the last optimization iteration of run 1-E39.

\begin{tabular}{l|rrrrr} 
Set & \multicolumn{1}{|c}{$\boldsymbol{A}_{\mathrm{zz}}$} & \multicolumn{1}{c}{$\boldsymbol{\alpha}$} & \multicolumn{1}{c}{$/^{\circ}$} & \multicolumn{1}{c}{$\boldsymbol{\beta} /{ }^{\circ}$} & \multicolumn{1}{c}{$\boldsymbol{\gamma} /^{\circ}$} \\
\hline set 1 & $-7.039 \mathrm{e}-04$ & $4.508 \mathrm{e}-01$ & 106.614 & 54.081 & 132.169 \\
set 2 & $8.750 \mathrm{e}-04$ & $5.101 \mathrm{e}-01$ & 104.966 & 126.999 & 161.873 \\
set 3 & $-1.280 \mathrm{e}-03$ & $3.819 \mathrm{e}-01$ & 173.038 & 101.759 & 132.569 \\
set 4 & $-5.380 \mathrm{e}-04$ & $5.827 \mathrm{e}-01$ & 170.093 & 118.722 & 130.653 \\
set 5 & $-5.554 \mathrm{e}-04$ & $5.716 \mathrm{e}-01$ & 3.628 & 117.657 & 126.624 \\
set 6 & $-7.847 \mathrm{e}-04$ & $2.740 \mathrm{e}-01$ & 82.142 & 91.507 & 139.637
\end{tabular}

\section{Isopinocampheol run 1-F}

\section{Structure generation via vector addition}

Additionally to the redundant internal coordinates algorithm the optimization was performed using the non-default vector addition algorithm. The orientations obtained are summarized in the tables below. Changes of the Euler angles compared to the literature data listed above are due to the change of the initial reference frames (TITANIA used the principle axis system of the molecule) and the added errors. These differences are addressed in table S-80 (section 2.4.2). 
Table S-73: Orientational data of IPC 1 obtained by TITANIA on the last optimization iteration for the run 1-F11 when using the non-default vector addition algorithm.

\begin{tabular}{l|rcrrr} 
Set & \multicolumn{1}{|c}{$\boldsymbol{A}_{\mathrm{zz}}$} & \multicolumn{1}{c}{} & \multicolumn{1}{c}{$\boldsymbol{\alpha} /{ }^{\circ}$} & \multicolumn{1}{c}{$\boldsymbol{\beta} /{ }^{\circ}$} & \multicolumn{1}{c}{$\boldsymbol{\gamma}{ }^{\circ}$} \\
\hline set 1 & $-7.986 \mathrm{e}-04$ & $3.230 \mathrm{e}-01$ & 2.066 & 137.336 & 110.782 \\
set 2 & $1.001 \mathrm{e}-03$ & $3.944 \mathrm{e}-01$ & 101.511 & 87.663 & 6.560 \\
set 7 & $-7.037 \mathrm{e}-04$ & $2.935 \mathrm{e}-01$ & 166.537 & 124.515 & 109.441 \\
set 8 & $1.025 \mathrm{e}-03$ & $4.540 \mathrm{e}-01$ & 73.463 & 90.888 & 176.144 \\
set 9 & $1.028 \mathrm{e}-03$ & $4.314 \mathrm{e}-01$ & 69.241 & 88.352 & 178.191 \\
set 10 & $9.506 \mathrm{e}-04$ & $4.538 \mathrm{e}-01$ & 106.320 & 88.585 & 5.534
\end{tabular}

Table S-74: Orientational data of IPC 1 obtained by TITANIA on the last optimization iteration of run 1-F17 when using the non-default vector addition algorithm.

\begin{tabular}{l|rcrrr} 
Set & \multicolumn{1}{|c}{$\boldsymbol{A}_{\mathrm{zz}}$} & \multicolumn{1}{c}{} & $\boldsymbol{\alpha} /{ }^{\circ}$ & \multicolumn{1}{c}{$\boldsymbol{\beta} /{ }^{\circ}$} & \multicolumn{1}{c}{$\boldsymbol{\gamma}{ }^{\circ}$} \\
\hline set 1 & $5.172 \mathrm{e}-04$ & $3.569 \mathrm{e}-01$ & 100.110 & 168.760 & 103.421 \\
set 2 & $-4.900 \mathrm{e}-04$ & $6.643 \mathrm{e}-01$ & 114.304 & 71.029 & 126.469 \\
set 7 & $4.518 \mathrm{e}-04$ & $3.707 \mathrm{e}-01$ & 115.334 & 175.073 & 82.319 \\
set 8 & $-6.387 \mathrm{e}-04$ & $3.458 \mathrm{e}-01$ & 116.715 & 66.886 & 135.240 \\
set 9 & $-6.327 \mathrm{e}-04$ & $4.583 \mathrm{e}-01$ & 108.846 & 65.639 & 135.803 \\
set 10 & $-5.175 \mathrm{e}-04$ & $5.732 \mathrm{e}-01$ & 110.411 & 70.972 & 127.709
\end{tabular}

Table S-75: Orientational data of IPC 1 obtained by TITANIA on the last optimization iteration of run 1-F23 when using the non-default vector addition algorithm.

\begin{tabular}{l|rcrrr} 
Set & \multicolumn{1}{c}{$\boldsymbol{A}_{\text {zz }}$} & $\boldsymbol{R}$ & $\boldsymbol{\alpha} /{ }^{\circ}$ & \multicolumn{1}{c}{$\boldsymbol{\beta} /^{\circ}$} & \multicolumn{1}{c}{$\boldsymbol{\gamma}{ }^{\circ}$} \\
\hline set 1 & $-7.187 \mathrm{e}-04$ & $3.226 \mathrm{e}-01$ & 44.621 & 30.151 & 72.340 \\
set 2 & $8.070 \mathrm{e}-04$ & $6.100 \mathrm{e}-01$ & 64.226 & 105.260 & 28.398 \\
set 7 & $-4.776 \mathrm{e}-04$ & $5.014 \mathrm{e}-01$ & 80.383 & 37.385 & 58.352 \\
set 8 & $8.589 \mathrm{e}-04$ & $6.540 \mathrm{e}-01$ & 67.644 & 101.645 & 25.270 \\
set 9 & $8.942 \mathrm{e}-04$ & $6.283 \mathrm{e}-01$ & 68.959 & 103.619 & 24.495 \\
set 10 & $7.606 \mathrm{e}-04$ & $6.647 \mathrm{e}-01$ & 65.988 & 104.884 & 27.058
\end{tabular}


Table S-76: Orientational data of IPC 1 obtained by TITANIA on the last optimization iteration of run 1-F31 when using the non-default vector addition algorithm.

\begin{tabular}{l|rcrrr} 
Set & \multicolumn{1}{|c}{$\boldsymbol{A}_{\text {zz }}$} & \multicolumn{1}{c}{$\boldsymbol{c}$} & \multicolumn{1}{c}{$\boldsymbol{\alpha}{ }^{\circ}$} & \multicolumn{1}{c}{$\boldsymbol{\beta} /{ }^{\circ}$} & \multicolumn{1}{c}{$\boldsymbol{\gamma} /^{\circ}$} \\
\hline set 1 & $-6.325 \mathrm{e}-04$ & $1.540 \mathrm{e}-01$ & 164.453 & 57.070 & 135.066 \\
set 2 & $6.561 \mathrm{e}-04$ & $4.912 \mathrm{e}-01$ & 39.950 & 85.192 & 31.400 \\
set 7 & $-4.383 \mathrm{e}-04$ & $1.960 \mathrm{e}-01$ & 134.982 & 69.883 & 146.908 \\
set 8 & $6.903 \mathrm{e}-04$ & $6.131 \mathrm{e}-01$ & 48.772 & 89.446 & 33.466 \\
set 9 & $6.647 \mathrm{e}-04$ & $5.898 \mathrm{e}-01$ & 48.406 & 85.773 & 31.000 \\
set 10 & $6.459 \mathrm{e}-04$ & $5.499 \mathrm{e}-01$ & 40.665 & 86.226 & 31.143
\end{tabular}

Table S-77: Orientational data of IPC 1 obtained by TITANIA on the last optimization iteration of run 1-F39 when using the non-default vector addition algorithm.

\begin{tabular}{l|rcrrr} 
Set & \multicolumn{1}{|c}{$\boldsymbol{A}_{\text {zz }}$} & \multicolumn{1}{c}{} & \multicolumn{1}{c}{$\boldsymbol{\alpha}{ }^{\circ}$} & \multicolumn{1}{c}{$\boldsymbol{\beta} /{ }^{\circ}$} & \multicolumn{1}{c}{$\boldsymbol{\gamma}{ }^{\circ}$} \\
\hline set 1 & $-6.493 \mathrm{e}-04$ & $2.931 \mathrm{e}-01$ & 83.540 & 45.197 & 133.570 \\
set 2 & $7.685 \mathrm{e}-04$ & $4.741 \mathrm{e}-01$ & 107.215 & 120.702 & 156.138 \\
set 7 & $-5.236 \mathrm{e}-04$ & $3.958 \mathrm{e}-01$ & 89.022 & 59.785 & 145.760 \\
set 8 & $8.758 \mathrm{e}-04$ & $4.584 \mathrm{e}-01$ & 106.274 & 116.057 & 156.547 \\
set 9 & $8.684 \mathrm{e}-04$ & $4.575 \mathrm{e}-01$ & 103.184 & 116.835 & 158.978 \\
set 10 & $7.645 \mathrm{e}-04$ & $5.018 \mathrm{e}-01$ & 110.056 & 117.807 & 159.900
\end{tabular}




\subsubsection{Change in Orientation}

\section{$1-\mathrm{D}$}

Table S-78: Generalized angle $\beta$ enclosed by the reference structure of IPC and the structure obtained by TITANIA (using the non-default vector addition algorithm) from the last optimization step of the runs 1-D. The data were obtained by transformation of the respective structures to a common reference frame, followed by recalculating the alignment tensors with the inhouse software RDC@hotFCHT. ${ }^{[9]}$

\begin{tabular}{l|rrrrr} 
& \multicolumn{5}{|c}{$\boldsymbol{\beta} /{ }^{\circ}$} \\
Set & 1-D11 & $\mathbf{1 - D 1 7}$ & $\mathbf{1 - D 2 3}$ & $\mathbf{1 - D 3 1}$ & $\mathbf{1 - D 3 9}$ \\
\hline set 1 & 30.946 & 15.144 & 9.845 & 9.685 & 7.362 \\
set 2 & 50.402 & 20.464 & 29.090 & 20.140 & 14.040 \\
set 3 & 33.763 & 12.271 & 4.070 & 25.837 & 8.092 \\
set 4 & 39.991 & 7.758 & 6.975 & 15.387 & 8.185 \\
set 5 & 15.312 & 7.121 & 7.499 & 13.342 & 9.721 \\
set 6 & 17.512 & 5.726 & 8.002 & 25.098 & 8.446 \\
set 7 & 24.971 & 11.795 & 12.735 & 36.093 & 6.718 \\
set 8 & 41.949 & 6.681 & 3.395 & 14.813 & 10.582 \\
set 9 & 60.050 & 23.234 & 16.183 & 56.141 & 9.593 \\
set 10 & 38.598 & 15.734 & 8.275 & 19.042 & 6.990 \\
set 11 & 22.213 & 8.194 & 5.626 & 23.012 & 10.864 \\
set 12 & 17.809 & 8.714 & 11.250 & 17.496 & 8.847 \\
set 13 & 12.005 & 15.378 & 7.849 & 23.905 & 11.418 \\
set 14 & 23.741 & 25.161 & 18.718 & 24.281 & 12.408 \\
set 15 & 38.101 & 16.293 & 13.371 & 15.462 & 6.005 \\
set 16 & 18.983 & 11.337 & 10.441 & 8.505 & 9.386 \\
set 17 & 37.170 & 8.983 & 8.302 & 12.882 & 6.654 \\
set 18 & 17.004 & 7.385 & 6.051 & 29.541 & 11.212 \\
set 19 & 44.102 & 11.605 & 5.659 & 12.636 & 8.456 \\
set 20 & 13.838 & 13.730 & 10.206 & 19.869 & 10.911
\end{tabular}




\section{$1-\mathrm{E}$}

Table S-79: Generalized angle $\beta$ enclosed by the reference structure of IPC and the structure obtained by TITANIA (using the non-default vector addition algorithm) from the last optimization step of the runs 1-E. The data were obtained by transformation of the respective structures to a common reference frame, followed by recalculating the alignment tensors with the inhouse software RDC@hotFCHT. ${ }^{[9]}$

\begin{tabular}{l|rrrrr} 
& \multicolumn{5}{|c}{$\boldsymbol{\beta} /{ }^{\circ}$} \\
Set & 1-E11 & 1-E17 & 1-E23 & 1-E31 & 1-E39 \\
\hline set 1 & 6.038 & 10.846 & 4.915 & 4.829 & 12.146 \\
set 2 & 4.647 & 11.171 & 7.822 & 5.393 & 8.713 \\
set 3 & 9.215 & 9.386 & 2.881 & 2.354 & 15.258 \\
set 4 & 7.580 & 5.549 & 4.465 & 3.928 & 17.104 \\
set 5 & 11.577 & 3.545 & 4.774 & 2.920 & 11.867 \\
set 6 & 7.147 & 11.098 & 2.417 & 1.879 & 16.664
\end{tabular}

\section{$1-\mathrm{F}$}

Table S-80: Generalized angle $\beta$ enclosed by the reference structure of IPC and the structure obtained by TITANIA (using the non-default vector addition algorithm) from the last optimization step of the runs $\mathbf{1 - F}$. The data were obtained by transformation of the respective structures to a common reference frame, followed by recalculating the alignment tensors with the inhouse software RDC@hotFCHT. ${ }^{[9]}$

\begin{tabular}{l|ccccc} 
& \multicolumn{5}{|}{$\boldsymbol{\beta} /{ }^{\circ}$} \\
Set & 1-F11 & 1-F17 & 1-F23 & 1-F31 & 1-F39 \\
\hline set 1 & 123.585 & 83.564 & 81.269 & 30.297 & 23.402 \\
set 2 & 72.325 & 59.705 & 80.800 & 64.861 & 23.772 \\
set 7 & 113.151 & 82.807 & 99.972 & 38.546 & 22.152 \\
set 8 & 64.246 & 64.948 & 73.213 & 57.898 & 18.615 \\
set 9 & 75.126 & 62.949 & 80.406 & 58.654 & 21.404 \\
set 10 & 75.531 & 63.720 & 78.106 & 60.904 & 21.482
\end{tabular}

As before for the redundant internal coordinates algorithm 1-F also does not converge when using the non-default vector addition algorithm.

The shown $\beta$ angles describe the difference of the alignment tensors calculated using the reference structure and the final structure of the TITANIA runs. When comparing the results to the $\beta$ angles obtained from the redundant internal coordinates algorithm it becomes clear that the angles are 
larger for the vector addition algorithms. The reason for this behavior are the missing restraints for the vector addition algorithms. This can lead to a collective motion of the RDC vector orientations as discussed in the main text for run 1-E39. In such a case the differences in the orientations of the structures compared will always be larger due to structural distortions. 


\subsubsection{Optimization Trajectories}

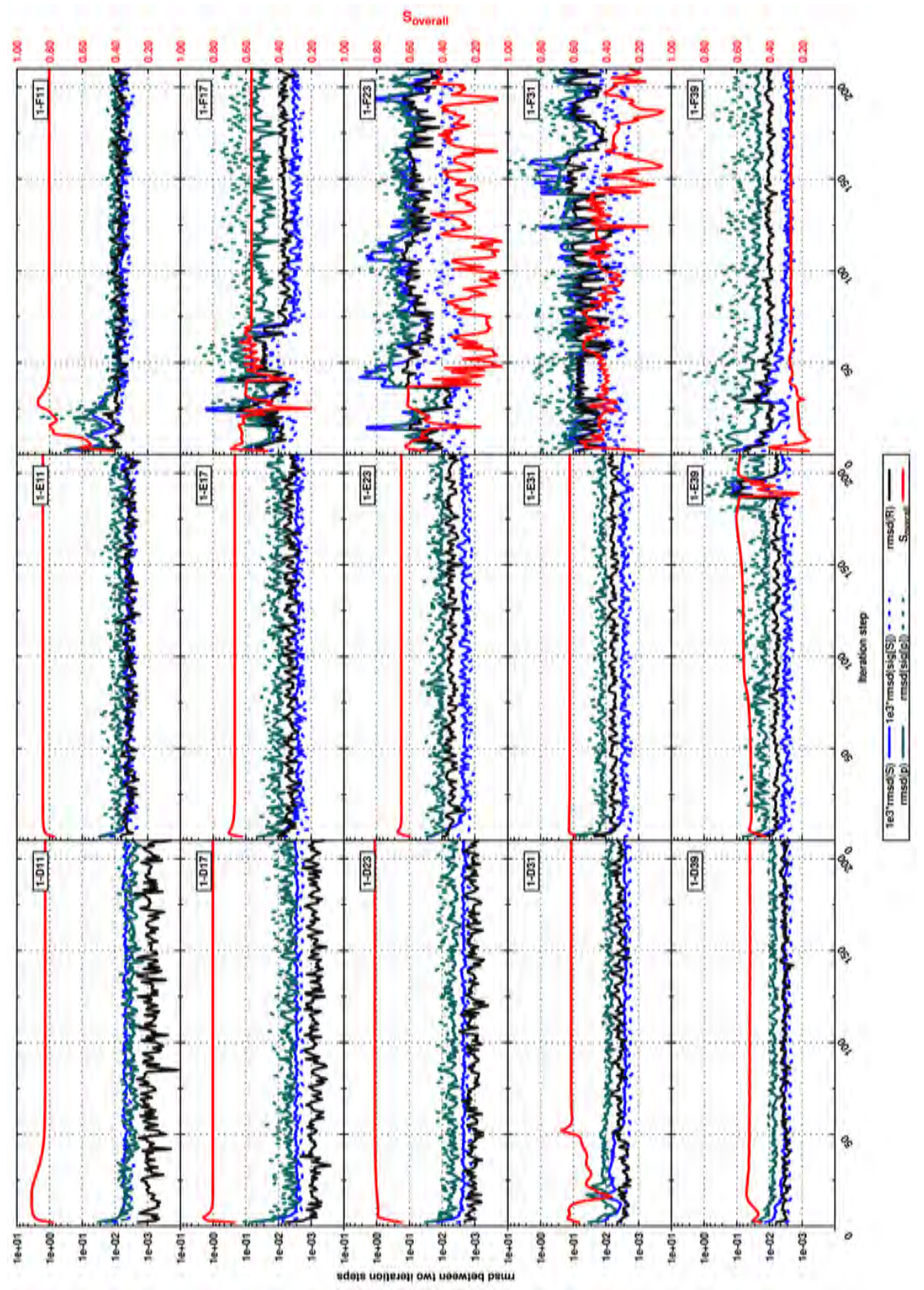

Figure S-17: Trajectory of the Monte-Carlo $\mathrm{rmsds}$ obtained for the individual runs of the setups 1-D, E and F. Shown are the data obtained by the vector addition algorithm. 


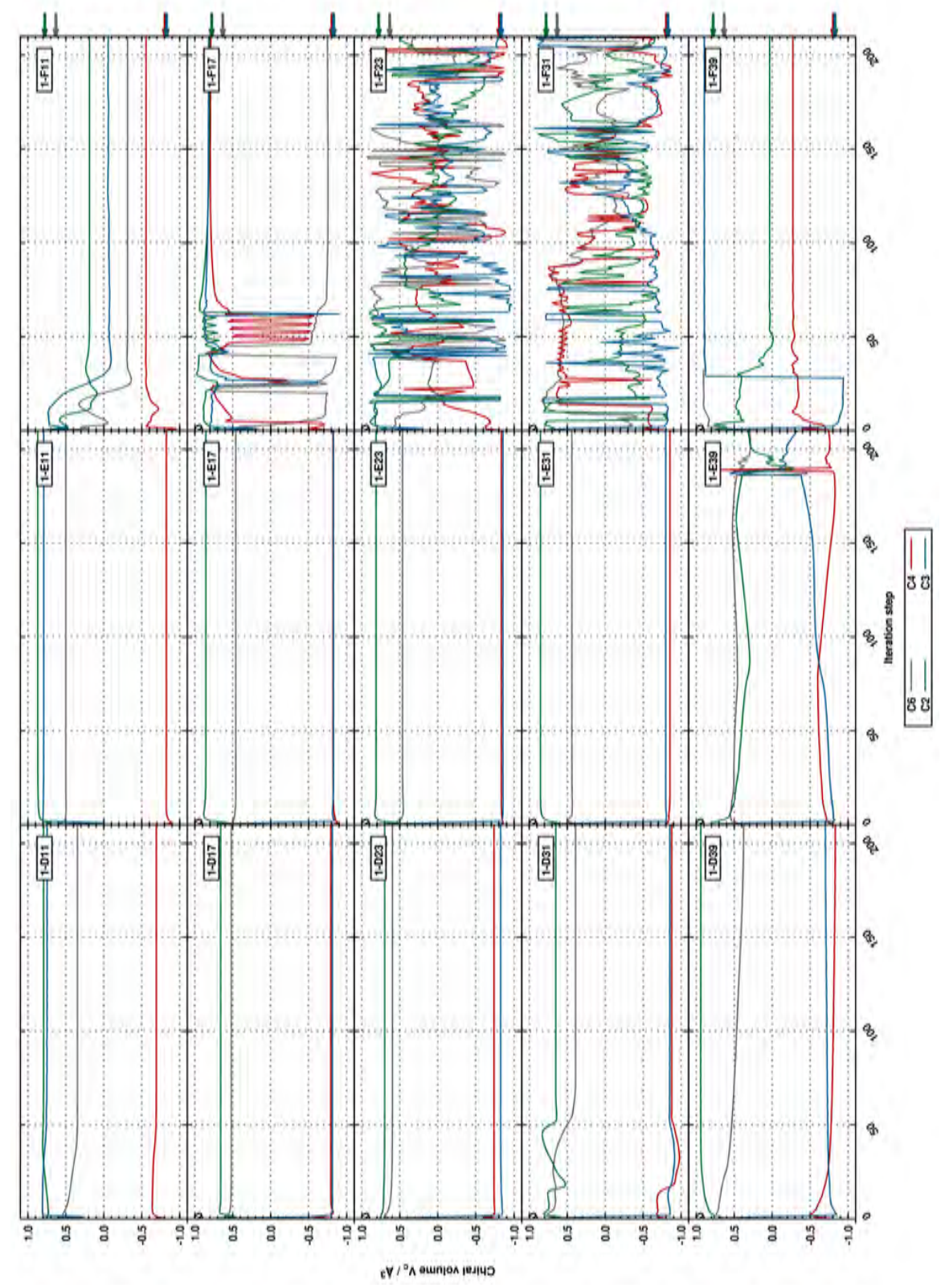

Figure S-18: Trajectory of the normalized chiral volumes obtained for the individual runs of the setups $\mathbf{1 - D}, \mathbf{E}$ and $\mathbf{F}$. Shown are the data obtained by the vector addtion algorithm. The arrows at the top indicate the values of the reference structure (correct configuration). 
The vector addition algorithm shows rather smooth trajectories for the setups 1-D and 1-E. The reason for this is that only RDC vectors are optimized and the scaffold is retained. In the case of 1-E39 this can lead to a strange behavior, where the RDC vectors show a collective movement around the fixed structure. At some point (around iteration 190) this leads to an instant rearrangement. Still the correct relative configuration was found for this run.

As expected the setup 1-F does not show convergence. A change of algorithms cannot help if the data quality is not sufficient. 


\subsection{RDC list}

Table S-81: List of all RDCs used in the runs of $\mathbf{1 - A}, \mathbf{B}$ and $\mathbf{C}$.

\begin{tabular}{|c|c|c|c|c|c|}
\hline RDC pair & $11 \mathrm{RDCs}$ & 17 RDCs & $23 \mathrm{RDCs}$ & $31 \mathrm{RDCs}$ & 39 RDCs \\
\hline C1-H1 & $\mathrm{X}$ & $\mathrm{x}$ & $\mathrm{X}$ & $\mathrm{X}$ & $\mathrm{X}$ \\
\hline C2-H2 & $\mathrm{X}$ & $\mathrm{X}$ & $\mathrm{X}$ & $\mathrm{X}$ & $\mathrm{X}$ \\
\hline C3-H3 & $\mathrm{x}$ & $\mathrm{x}$ & $\mathrm{x}$ & $\mathrm{x}$ & $\mathrm{x}$ \\
\hline C4-H4a & $\mathrm{X}$ & $\mathrm{X}$ & $\mathrm{x}$ & $\mathrm{x}$ & $\mathrm{x}$ \\
\hline C4-H4s & $\mathrm{x}$ & $\mathrm{x}$ & $\mathrm{X}$ & $\mathrm{x}$ & $\mathrm{x}$ \\
\hline C5-H5 & $\mathrm{X}$ & $\mathrm{X}$ & $\mathrm{X}$ & $\mathrm{X}$ & $\mathrm{X}$ \\
\hline C7-H7a & $\mathrm{X}$ & $\mathrm{X}$ & $\mathrm{x}$ & $\mathrm{x}$ & $\mathrm{x}$ \\
\hline C7-H7s & $\mathrm{x}$ & $\mathrm{x}$ & $\mathrm{x}$ & $\mathrm{x}$ & $\mathrm{x}$ \\
\hline C10-C2 & $\mathrm{X}$ & $\mathrm{X}$ & $\mathrm{x}$ & $\mathrm{x}$ & $\mathrm{X}$ \\
\hline C6-C8 & $\mathrm{X}$ & $\mathrm{x}$ & $\mathrm{x}$ & $\mathrm{x}$ & $\mathrm{x}$ \\
\hline C6-C9 & $\mathrm{x}$ & $\mathrm{x}$ & $\mathrm{x}$ & $\mathrm{x}$ & $\mathrm{x}$ \\
\hline H1-H2 & & $\mathrm{x}$ & $\mathrm{x}$ & $\mathrm{x}$ & $\mathrm{x}$ \\
\hline H1-H3 & & & & $\mathrm{x}$ & $\mathrm{x}$ \\
\hline H1-H4a & & & & & $\mathrm{x}$ \\
\hline H1-H4s & & & & $\mathrm{x}$ & $\mathrm{x}$ \\
\hline H1-H5 & & & & $\mathrm{x}$ & $\mathrm{x}$ \\
\hline H1-H7a & & & $\mathrm{x}$ & & $\mathrm{x}$ \\
\hline H1-H7s & & $\mathrm{X}$ & $\mathrm{x}$ & $\mathrm{x}$ & $\mathrm{x}$ \\
\hline H2-H3 & & $\mathrm{x}$ & $\mathrm{x}$ & $\mathrm{x}$ & $\mathrm{x}$ \\
\hline H2-H4a & & & & $\mathrm{x}$ & $\mathrm{x}$ \\
\hline H2-H4s & & & & & $\mathrm{x}$ \\
\hline H2-H5 & & & & & $\mathrm{x}$ \\
\hline H2-H7a & & & & & $\mathrm{x}$ \\
\hline H2-H7s & & & & $\mathrm{x}$ & $\mathrm{x}$ \\
\hline H3-H4a & & & $\mathrm{x}$ & $\mathrm{x}$ & $\mathrm{x}$ \\
\hline H3-H4s & & $\mathrm{X}$ & $\mathrm{x}$ & $\mathrm{x}$ & $\mathrm{x}$ \\
\hline H3-H5 & & & & & $\mathrm{x}$ \\
\hline H3-H7a & & & & $\mathrm{x}$ & $\mathrm{x}$ \\
\hline H3-H7s & & & & & $\mathrm{x}$ \\
\hline H4a-H4s & & & $\mathrm{x}$ & $\mathrm{x}$ & $\mathrm{x}$ \\
\hline H4a-H5 & & & $\mathrm{x}$ & $\mathrm{x}$ & $\mathrm{x}$ \\
\hline H4a-H7a & & & & $\mathrm{x}$ & $\mathrm{x}$ \\
\hline H4a-H7s & & & & & $\mathrm{X}$ \\
\hline H4s-H5 & & $\mathrm{X}$ & $\mathrm{x}$ & $\mathrm{x}$ & $\mathrm{x}$ \\
\hline H4s-H7a & & & & $\mathrm{x}$ & $\mathrm{x}$ \\
\hline H4s-H7s & & & & $\mathrm{x}$ & $\mathrm{x}$ \\
\hline H5-H7a & & $\mathrm{X}$ & $\mathrm{x}$ & $\mathrm{x}$ & $\mathrm{x}$ \\
\hline H5-H7s & & & $\mathrm{x}$ & $\mathrm{x}$ & $\mathrm{x}$ \\
\hline H7a-H7s & & & $\mathrm{x}$ & $\mathrm{x}$ & $\mathrm{x}$ \\
\hline
\end{tabular}


2.6 Final structures
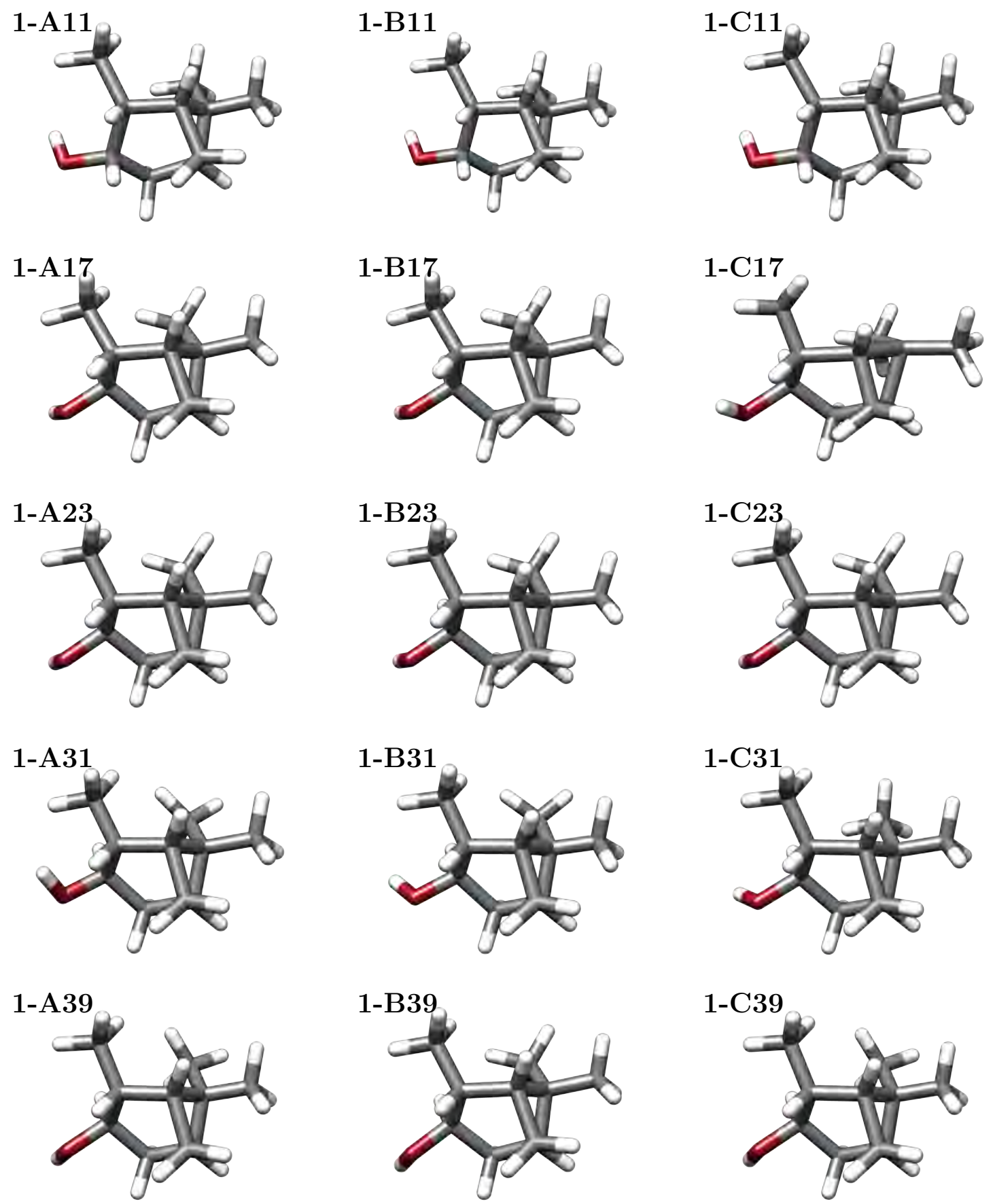

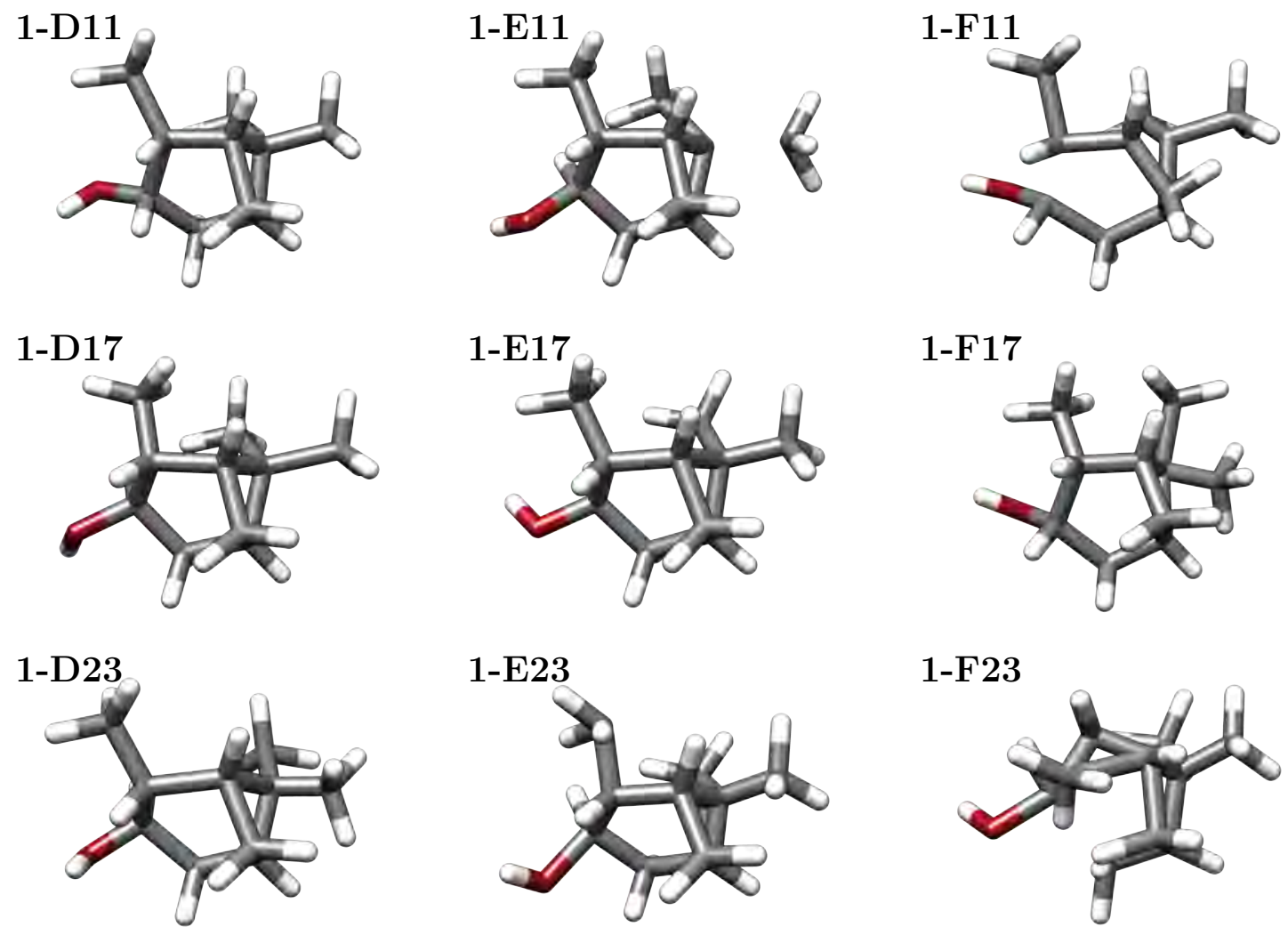

$1-\mathrm{E} 23$

$1-\mathrm{F} 23$
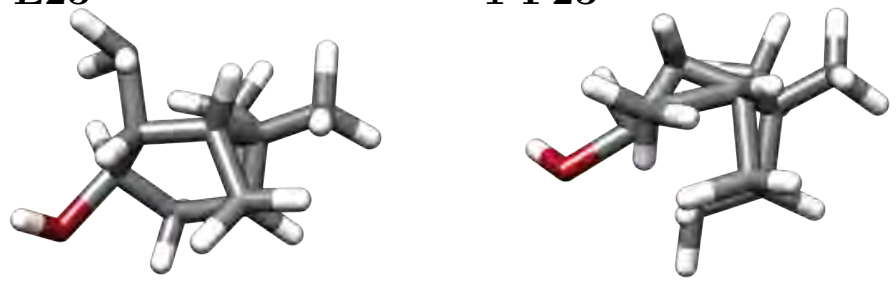

1-D31

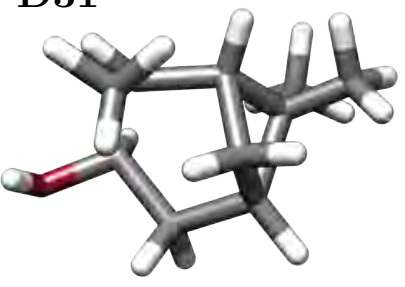

1-E31

1-F31
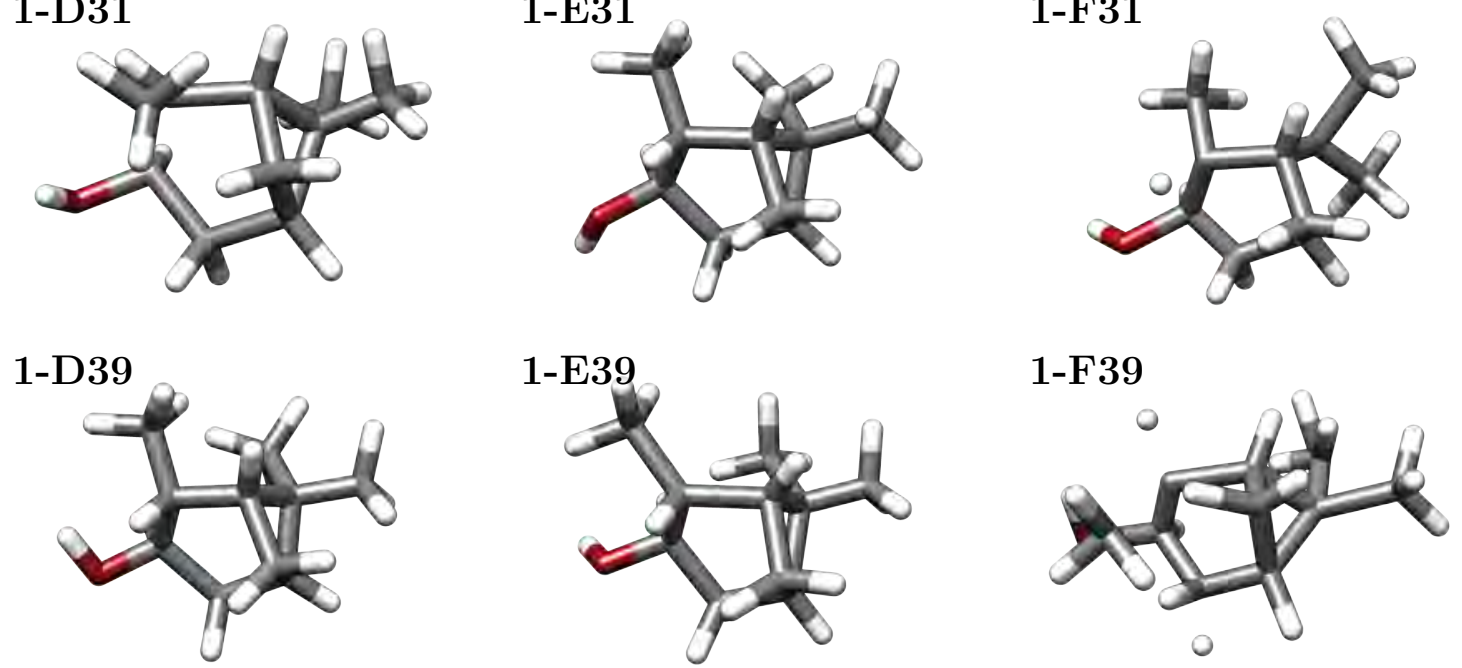

The xyz coordinates of the final structures are all available in the supplementary material. 


\section{Tubocurarine (2)}

Tubocurarine was optimized in two setups, which were built on the same eight randomly generated alignment tensors. The goal was to investigate the possibility of optimizing a rather complex structure with conformational flexibility and to investigate the impact of heterogeneities on the result. Therefore the data will differ from those in section 2. For a better understanding the structure containing all RDCs (in addition to the respective RDC list in section 3.3) is given here.

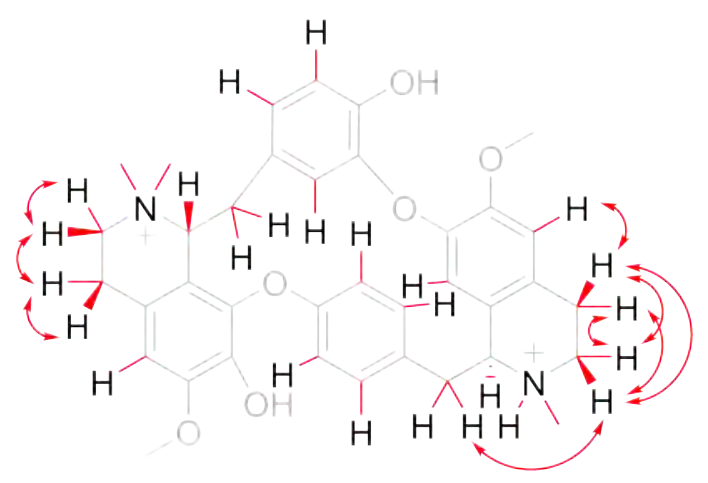

2

Figure S-19: Structure of tubocurarine $\mathbf{2}$ with all RDCs used for the TITANIA optimization marked in red. 


\subsection{SECONDA eigenmodes}

The eigenmodes of $\mathbf{2}-\mathbf{A}$ and $\mathbf{2}-\mathbf{B}$ give additional insights into the homogeneity and heterogeneity of the RDC data. As expected from literature the eigenmodes 1 to 5 obtained from the homogeneous setup 2-A have equally distributed elements with no spikes. This is also expected from figure 10 in the main text showing the SECONDA plot of $\mathbf{2 - A}$ and $\mathbf{2 - B}$, in which the collectivities of $\mathbf{2 - A}$ are all larger than $50 \%$. This behavior is changed drastically by adding heterogeneity in setup 2-B. Here most eigenmodes contain large spikes, which can all be assigned to ${ }^{1} D_{\mathrm{CN}}$ couplings. These couplings have the largest relative error in the data set. This is due to the fact that the added random numbers were not scaled down for these RDCs despite the low magnitude of the corresponding $D_{\max }$. The effect of the heterogeneity is even large enough that the principle variance $\lambda_{4, \mathbf{2}-\mathbf{A}}$ and the corresponding eigenmode $\left|4_{2-\mathbf{A}}\right\rangle$ coincide with $\lambda_{5, \mathbf{2}-\mathbf{B}}$ and $\left|5_{\mathbf{2}-\mathbf{B}}\right\rangle$ (see gray trace in fig. S20). This means that a principle variance and eigenmode which is mainly elicited by the synthetic error $\left(\lambda_{4, \mathbf{2}-\mathbf{B}}\right)$ is within the first five eigenvalues, thus implying a shift of eigenvalues. Additionally $\lambda_{5, \mathbf{2}-\mathbf{A}}$ and $\left|5_{\mathbf{2}-\mathbf{A}}\right\rangle$ are very similar to $\lambda_{7, \mathbf{2}-\mathbf{B}}$ and $\left|7_{\mathbf{2}-\mathbf{B}}\right\rangle$ (see gray trace in fig. S-20). 


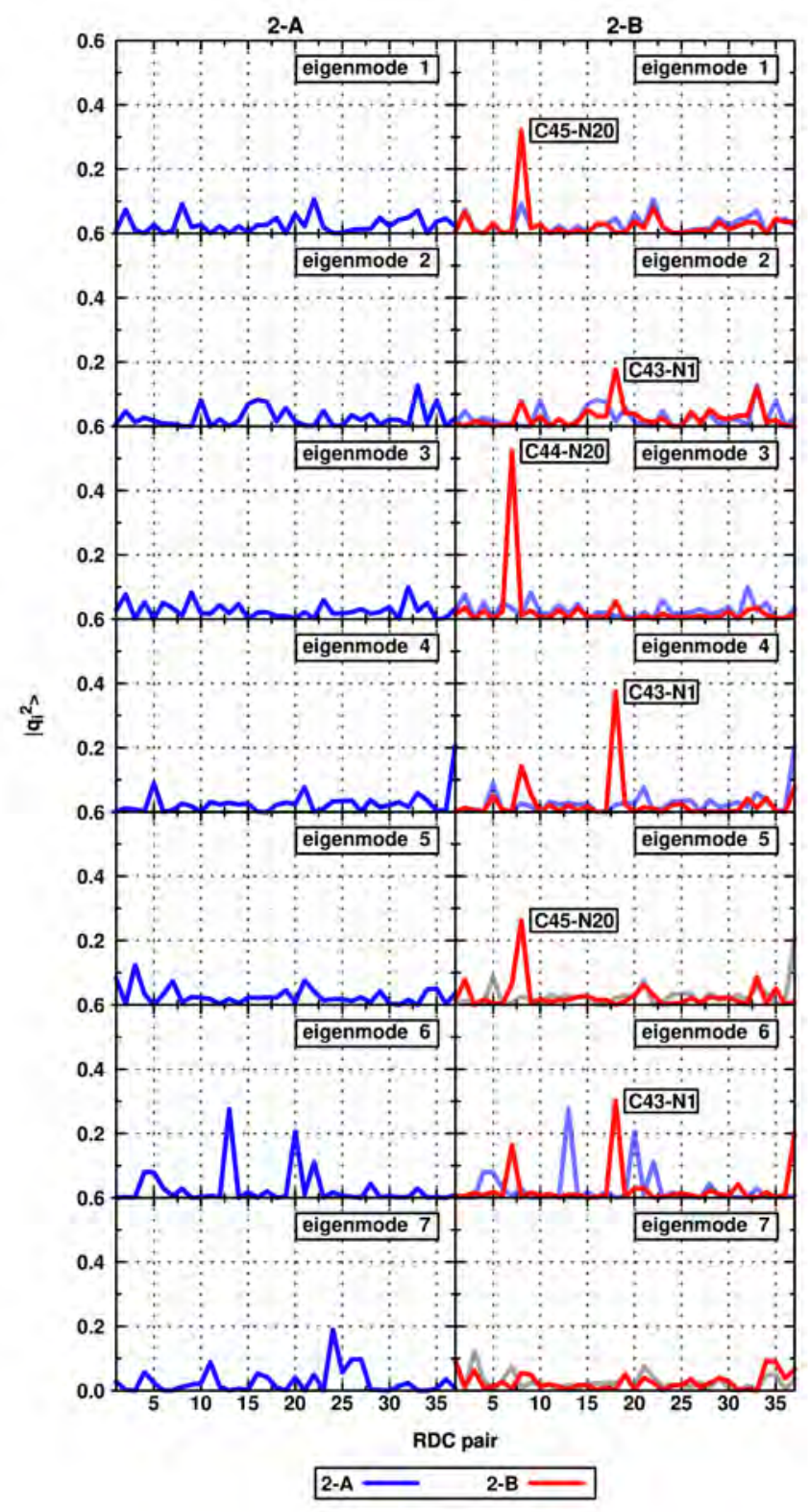

Figure S-20: Eigenmodes of the setups 2-A (blue) and 2-B (red) obtained from SECONDA. Note that in the panel on the right (2-B) a superposition with the corresponding eigenmodes of $\mathbf{2}-\mathbf{A}$ is shown. For eigenmodes $\left|5_{\mathbf{2}-\mathbf{B}}\right\rangle$ and $\left|7_{\mathbf{2}-\mathbf{B}}\right\rangle$ the corresponding eigenmode of $\mathbf{2 - \mathbf { A }}$ is $\left|4_{\mathbf{2}-\mathbf{A}}\right\rangle$ and $\left|5_{\mathbf{2}-\mathbf{A}}\right\rangle$, respectively. These are shown in gray. 


\subsection{Orientations}

\subsubsection{Tubocurarine run 2-A}

The RDCs of run 2-A were calculated by the orientations summarized in table S-82. These orientations were generated from random numbers, where $A_{\mathrm{zz}}$ was constrained to be lower than $1 \mathrm{e}-2$.

Table S-82: Orientational data used for the artifical RDC sets of run 2-A and $2-\mathbf{B}$.

\begin{tabular}{l|rcrrr} 
Set & \multicolumn{1}{c}{$\boldsymbol{A}_{\mathrm{zz}}$} & $\boldsymbol{R}$ & \multicolumn{1}{c}{$\boldsymbol{\alpha} /{ }^{\circ}$} & \multicolumn{1}{c}{$\boldsymbol{\beta} /{ }^{\circ}$} & \multicolumn{1}{c}{$\boldsymbol{\gamma} /{ }^{\circ}$} \\
\hline set 1 & $-2.522 \mathrm{e}-03$ & $2.534 \mathrm{e}-01$ & 19.620 & 117.810 & 39.700 \\
set 2 & $7.391 \mathrm{e}-04$ & $4.661 \mathrm{e}-01$ & 71.810 & 75.570 & 146.000 \\
set 3 & $-7.096 \mathrm{e}-04$ & $6.177 \mathrm{e}-01$ & 66.370 & 138.590 & 116.000 \\
set 4 & $-8.076 \mathrm{e}-04$ & $5.195 \mathrm{e}-01$ & 61.600 & 154.440 & 119.000 \\
set 5 & $-1.580 \mathrm{e}-03$ & $3.812 \mathrm{e}-01$ & 148.030 & 117.480 & 149.500 \\
set 6 & $-8.315 \mathrm{e}-04$ & $6.143 \mathrm{e}-01$ & 142.480 & 103.210 & 156.400 \\
set 7 & $-7.961 \mathrm{e}-04$ & $3.370 \mathrm{e}-01$ & 45.600 & 118.300 & 131.000 \\
set 8 & $6.659 \mathrm{e}-04$ & $5.547 \mathrm{e}-01$ & 164.010 & 71.820 & 72.200
\end{tabular}

\section{Output orientations}

All orientiations of setup 2-A obtained from the full TITANIA optimization runs are listed in the following section. Changes of the Euler angles, compared to the input data listed above, are due to the change of the reference frames (TITANIA used the principle axis system of the molecule). The comparison of the input and output data is summarized in table S-85 (section 3.2.3). 
Table S-83: Orientational data of tubocurarine $\mathbf{2}$ obtained by TITANIA on the last optimization iteration for the runs $\mathbf{2}-\mathbf{A}$.

\begin{tabular}{l|rcrrr} 
Set & \multicolumn{1}{c}{$\boldsymbol{A}_{\text {zz }}$} & \multicolumn{1}{c}{$\boldsymbol{\alpha}$} & \multicolumn{1}{c}{$/^{\circ}$} & \multicolumn{1}{c}{$\boldsymbol{\beta} /{ }^{\circ}$} & \multicolumn{1}{c}{$\boldsymbol{\gamma}{ }^{\circ}$} \\
\hline set 1 & $-2.645 \mathrm{e}-03$ & $3.105 \mathrm{e}-01$ & 27.927 & 60.902 & 131.974 \\
set 2 & $7.445 \mathrm{e}-04$ & $4.327 \mathrm{e}-01$ & 67.523 & 112.378 & 33.395 \\
set 3 & $6.992 \mathrm{e}-04$ & $6.290 \mathrm{e}-01$ & 69.164 & 135.421 & 34.727 \\
set 4 & $-7.588 \mathrm{e}-04$ & $5.840 \mathrm{e}-01$ & 64.918 & 33.967 & 59.359 \\
set 5 & $-1.457 \mathrm{e}-03$ & $4.094 \mathrm{e}-01$ & 139.165 & 70.484 & 30.973 \\
set 6 & $7.758 \mathrm{e}-04$ & $6.192 \mathrm{e}-01$ & 171.343 & 134.193 & 107.367 \\
set 7 & $-7.646 \mathrm{e}-04$ & $3.274 \mathrm{e}-01$ & 49.797 & 70.676 & 46.730 \\
set 8 & $6.092 \mathrm{e}-04$ & $5.309 \mathrm{e}-01$ & 172.420 & 115.822 & 109.699
\end{tabular}

\subsubsection{Tubocurarine run 2-B}

The RDCs of run 2-B were generated from the $\mathbf{2}-\mathbf{A}$ RDCs with addition of Gaussian error $(\sigma=0.5 \mathrm{~Hz})$. All orientiations of setup 2-B obtained from the full TITANIA optimization runs are listed in the following section. Changes of the Euler angles compared to the literature data listed above are due to the change of the reference frames (TITANIA used the principle axis system of the molecule). The comparison of the input and output data is summarized in table S-85 (section 3.2.3).

Table S-84: Orientational data of tubocurarine $\mathbf{2}$ obtained by TITANIA on the last optimization iteration of run 2-B.

\begin{tabular}{l|rcrrr} 
Set & \multicolumn{1}{c}{$\boldsymbol{A}_{\mathrm{zz}}$} & \multicolumn{1}{c}{$\boldsymbol{\alpha}$} & \multicolumn{1}{c}{$\boldsymbol{\alpha}{ }^{\circ}$} & \multicolumn{1}{c}{$\boldsymbol{\beta} /{ }^{\circ}$} & \multicolumn{1}{c}{$\boldsymbol{\gamma} /^{\circ}$} \\
\hline set 1 & $-2.822 \mathrm{e}-03$ & $3.615 \mathrm{e}-01$ & 17.286 & 88.193 & 145.503 \\
set 2 & $6.736 \mathrm{e}-04$ & $4.686 \mathrm{e}-01$ & 34.365 & 92.548 & 32.461 \\
set 3 & $6.277 \mathrm{e}-04$ & $6.485 \mathrm{e}-01$ & 40.373 & 115.349 & 21.292 \\
set 4 & $-6.981 \mathrm{e}-04$ & $6.483 \mathrm{e}-01$ & 17.325 & 30.906 & 105.648 \\
set 5 & $-1.792 \mathrm{e}-03$ & $4.060 \mathrm{e}-01$ & 123.030 & 52.257 & 38.777 \\
set 6 & $9.544 \mathrm{e}-04$ & $6.394 \mathrm{e}-01$ & 137.002 & 141.092 & 78.246 \\
set 7 & $-7.975 \mathrm{e}-04$ & $2.826 \mathrm{e}-01$ & 24.163 & 64.957 & 64.493 \\
set 8 & $7.868 \mathrm{e}-04$ & $5.060 \mathrm{e}-01$ & 147.746 & 137.159 & 90.780
\end{tabular}




\subsubsection{Change in Orientations}

To quantify the similarity of the input and the output orientations a common reference frame has to be used. Therefore an all atom rmsd structure alignment was performed to match the TITANIA output structure with the reference structure (or its enantiomer). The alignment tensors of the transformed structures are recalculated and the differences in the orientations (TITANIA vs. reference) are expressed by the $\beta$ angle.

Table S-85: Generalized angle $\beta$ enclosed by the reference structure of tubocurarine and the structure obtained by TITANIA from the last optimization step of the runs $\mathbf{2 - A}$ and $\mathbf{2 - B}$. The data were obtained by transformation of the respective structures to a common reference frame, followed by recalculating the alignment tensors with the in-house software RDC@hotFCHT. ${ }^{[9]}$

\begin{tabular}{l|cc} 
& \multicolumn{2}{|c}{$\boldsymbol{\beta} /{ }^{\circ}$} \\
Set & 2-A & 2-B \\
\hline set 1 & 8.986 & 26.982 \\
set 2 & 14.368 & 43.365 \\
set 3 & 10.712 & 40.292 \\
set 4 & 14.001 & 48.061 \\
set 5 & 5.750 & 23.571 \\
set 6 & 9.489 & 36.148 \\
set 7 & 9.769 & 35.040 \\
set 8 & 12.558 & 30.525
\end{tabular}

The $\beta$ angles for comparison of the reference and final structure in run 2-A are induced by structural change. Additionally to fluctuations of the spherical angles (angular part of the RDC definition) the bond lengths and distances (radial part of the RDC definition) show deviations from the reference structure. These changes lead to differences in the alignment parameters. 2-B shows even higher $\beta$ angles, which are caused by the same fluctuations, but additionally heterogeneities in the RDCs come into play. This is even more pronounced by the fact, that the final $\mathbf{2 - B}$ structure shows large distortions in the methoxy groups, leading to a change in the process of finding a proper common reference frame. These distortions are marked in fig. S-21. 


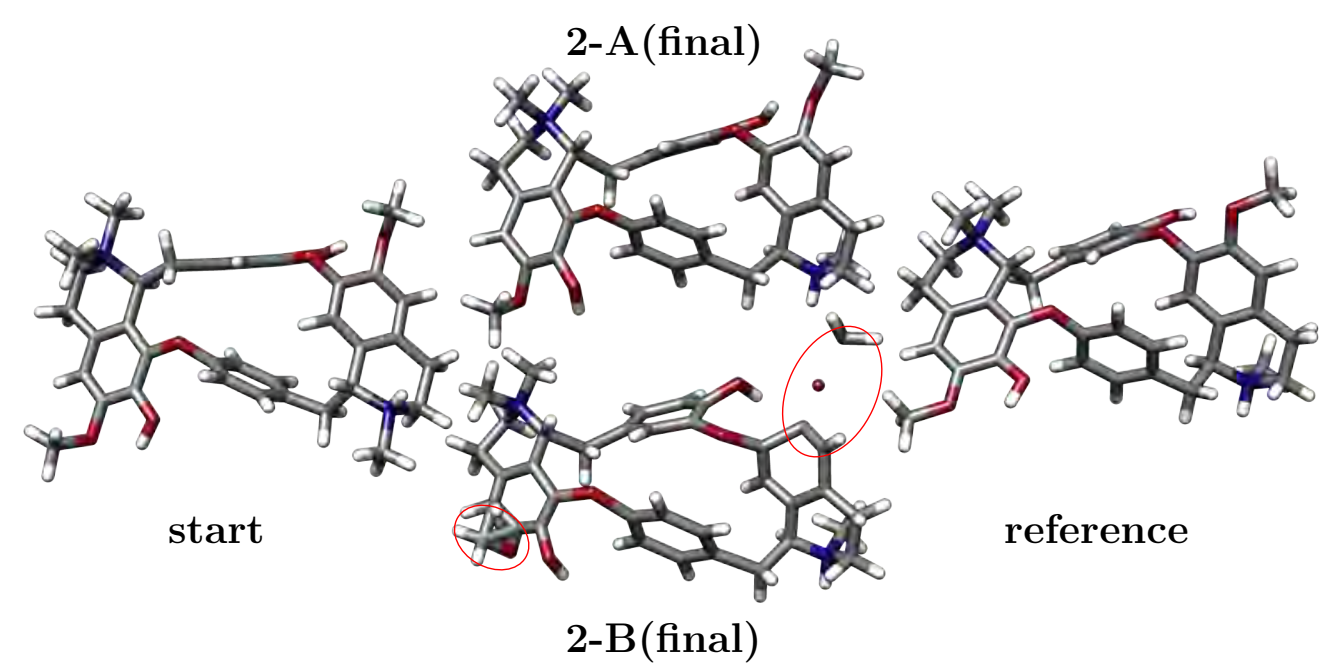

Figure S-21: Comparison of tubocurarine $\mathbf{2}$ structures. Shown are the start structure of the optimizations (left), the reference structure used of the prediction of the RDCs (right) and the final structures obtained from the TITANIA optimization runs (middle). In the structure of $\mathbf{2}-\mathbf{B}$ the methoxy groups marked showed large distortions which did not disturb the overall structure optimization.

Table S-86: Generalized angle $\beta$ enclosed by the orientations of tubocurarine $\mathbf{2}$ obtained by TITANIA in the last optimization step of the runs $\mathbf{2}-\mathbf{A}$ and 2-B. The structures were transformed into a common reference frame by all atom rmsd alignment. Additionally the $\beta$ angle was calculated from the reference structure to separate the change induced by errors.

\begin{tabular}{l|cc} 
& \multicolumn{2}{|c}{$\boldsymbol{\beta} /{ }^{\circ}$} \\
Set & TITANIA & Reference \\
\hline set 1 & 27.9157 & 10.545 \\
set 2 & 40.1463 & 12.704 \\
set 3 & 34.3988 & 6.933 \\
set 4 & 31.1403 & 14.173 \\
set 5 & 23.0264 & 2.6737 \\
set 6 & 33.5241 & 6.9610 \\
set 7 & 32.2838 & 4.0627 \\
set 8 & 18.0189 & 3.0128
\end{tabular}

The previously discussed $\beta$ angles in table S-85 (2-B) can again be found in table S-86 when comparing the final TITANIA structures. Separating the influence of the structure, as above, from the heterogeneities by using the 
reference structure (see column Reference in table S-86) relatively small $\beta$ angles are found. This means, that the difference of the individual $\mathbf{2 - A}$ and 2-B sets is mainly elicited by the changes in the mean structure.

Comparing the final structure of $\mathbf{2 - A}$ and the reference structure it becomes clear, that TITANIA is capable of a simultaneous interpretation of the RDC data in the context of conformational and configurational structure determination, as long as sufficient data is available.

\subsection{RDC list}

The RDC lists of the IPC $\mathbf{2}$ runs were equal for the respective sets of alignment media. All RDCs used for $\mathbf{2 - A}$ and $\mathbf{2 - B}$ are listed in table S-81.

Table S-87: List of all RDCs used in the runs of $\mathbf{2 - A}$ and $\mathbf{B}$.

\begin{tabular}{l|l} 
RDC pair & RDC pair \\
C21-H21a & C21-H21e \\
C22-H22a & C22-H22e \\
C25-H25 & C19-H19 \\
C44-N20 & C45-N20 \\
C18-H18r & C18-H18s \\
C31-H31 & C32-H32 \\
C34-H34 & C35-H35 \\
C36-H36r & C36-H36s \\
C6-H6 & C43-N1 \\
H1-N1 & C2-H2a \\
C2-H2e & C3-H3a \\
C3-H3e & C10-H10 \\
C7-H7 & C17-H17 \\
C15-H15 & C14-H14 \\
H21a-H21e & H22a-H22e \\
H21e-H22a & H3e-H7 \\
H2e-H3e & H2e-H3a \\
H2a-H3e & H2a-H3a \\
\hline H2a-H36r &
\end{tabular}




\section{Strychnine (3)}

Strychnine $\mathbf{3}$ is an example used to demonstrate the optimization of a highly fused carbon scaffold. Since previous examples already have shown that optimizations can still be performed when heterogeneities are present in the RDC data, we refrain from repeating to take this discussion again and perform the optimization only with artificial RDCs.

\subsection{SECONDA plot}

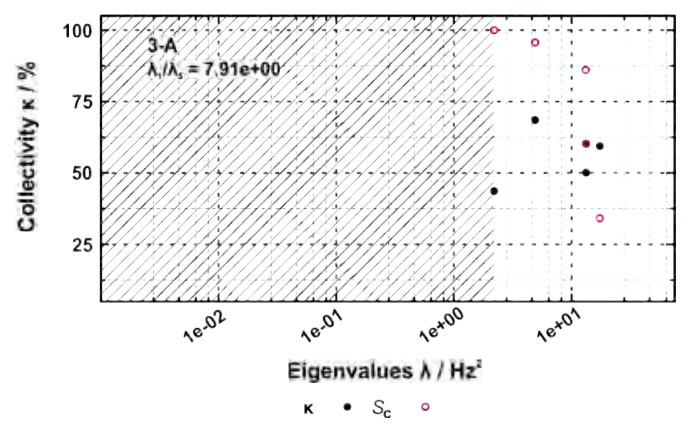

Figure S-22: SECONDA plot of strychnine run 3-A. Plotted are the collectivities $\kappa$ in respect to the eigenvalues $\lambda$ (black) of the RDC covariance matrix. In addition the cumulative sum (red) of the eigenvalues were plotted to measure the participation of the principle variances to the overall variance of the RDC matrix.

The SECONDA plot shows a linear independence of the setup, which is comparable to $\mathbf{1 - A}\left(\lambda_{1, \mathbf{1}-\mathbf{A}} / \lambda_{5, \mathbf{1}-\mathbf{A}}\right.$ between 5 and 9$)$. As expected no additional non-zero eigenvalues $\lambda_{\mathrm{n}}(\mathrm{n}>5)$ are observed since homogeneous back-calculated RDC data was used.

\subsection{Orientations}

\subsubsection{Strychnine run 3-A}

\section{Input orientations}

The RDCs of run 3-A were calculated by the orientations summarized in table S-88. 
Table S-88: Orientational data used for the artifical RDC sets of run 3-A.

\begin{tabular}{l|ccrrr} 
Set & $\boldsymbol{A}_{\mathrm{zz}}$ & $\boldsymbol{R}$ & \multicolumn{1}{c}{$\boldsymbol{\alpha} /{ }^{\circ}$} & \multicolumn{1}{c}{$\boldsymbol{\beta} /{ }^{\circ}$} & \multicolumn{1}{c}{$\boldsymbol{\gamma} /^{\circ}$} \\
\hline set 1 & $4.702 \mathrm{e}-04$ & $249 \mathrm{e}-01$ & 101.85 & -126.97 & -138.66 \\
set 2 & $5.529 \mathrm{e}-04$ & $281 \mathrm{e}-01$ & 90.67 & -11.21 & 87.58 \\
set 3 & $1.761 \mathrm{e}-04$ & $478 \mathrm{e}-01$ & 148.27 & -63.18 & 110.96 \\
set 4 & $9.310 \mathrm{e}-04$ & $408 \mathrm{e}-01$ & 22.86 & 122.49 & -12.90 \\
set 5 & $2.507 \mathrm{e}-04$ & $329 \mathrm{e}-01$ & 178.66 & -142.29 & 136.69 \\
set 6 & $2.094 \mathrm{e}-04$ & $517 \mathrm{e}-01$ & 132.02 & 62.69 & -147.29 \\
set 7 & $2.134 \mathrm{e}-04$ & $246 \mathrm{e}-01$ & 140.50 & -45.14 & -84.16 \\
set 8 & $4.079 \mathrm{e}-04$ & $628 \mathrm{e}-01$ & 115.52 & -87.76 & -135.31 \\
set 9 & $4.750 \mathrm{e}-04$ & $578 \mathrm{e}-01$ & 142.36 & 106.33 & -25.05 \\
set 10 & $5.166 \mathrm{e}-04$ & $486 \mathrm{e}-01$ & 71.08 & 173.66 & 91.56 \\
set 11 & $2.898 \mathrm{e}-04$ & $310 \mathrm{e}-01$ & 159.43 & -1.56 & -35.12
\end{tabular}

\section{Output orientations}

All orientiations of run 3-A obtained from the full TITANIA optimization runs. The orientations differ from table S-88 due to the change of the reference frame (TITANIA used the PAS of the inertia tensor). The comparison in the same reference frame can be found in table S-90 (section 4.2.2).

Table S-89: Orientational data of strychnine $\mathbf{3}$ obtained by TITANIA on the last optimization iteration for the runs $\mathbf{3 - A}$.

\begin{tabular}{l|ccrrr} 
Set & $\boldsymbol{A}_{\text {zz }}$ & \multicolumn{1}{c}{$\boldsymbol{R}$} & \multicolumn{1}{c}{$\boldsymbol{\alpha} /{ }^{\circ}$} & \multicolumn{1}{c}{$\boldsymbol{\beta} /{ }^{\circ}$} & \multicolumn{1}{c}{$\boldsymbol{\gamma} /^{\circ}$} \\
\hline set 1 & $5.681 \mathrm{e}-04$ & $3.097 \mathrm{e}-01$ & 100.083 & 127.472 & 126.526 \\
set 2 & $4.648 \mathrm{e}-04$ & $2.561 \mathrm{e}-01$ & 135.899 & 97.251 & 86.745 \\
set 3 & $1.724 \mathrm{e}-04$ & $4.639 \mathrm{e}-01$ & 21.351 & 76.388 & 140.959 \\
set 4 & $9.215 \mathrm{e}-04$ & $3.958 \mathrm{e}-01$ & 75.032 & 126.756 & 47.192 \\
set 5 & $2.600 \mathrm{e}-04$ & $3.487 \mathrm{e}-01$ & 79.325 & 153.659 & 57.116 \\
set 6 & $2.064 \mathrm{e}-04$ & $5.263 \mathrm{e}-01$ & 82.353 & 93.409 & 75.199 \\
set 7 & $2.105 \mathrm{e}-04$ & $2.432 \mathrm{e}-01$ & 118.222 & 4.328 & 148.826 \\
set 8 & $4.264 \mathrm{e}-04$ & $5.862 \mathrm{e}-01$ & 150.163 & 62.968 & 67.569 \\
set 9 & $4.755 \mathrm{e}-04$ & $5.694 \mathrm{e}-01$ & 3.303 & 124.333 & 24.379 \\
set 10 & $5.356 \mathrm{e}-04$ & $4.846 \mathrm{e}-01$ & 79.299 & 132.038 & 128.516 \\
set 11 & $2.938 \mathrm{e}-04$ & $3.565 \mathrm{e}-01$ & 145.537 & 139.275 & 125.117
\end{tabular}




\subsubsection{Change in Orientations}

To quantify the similarity of the input orientations with the output orientations a common reference frame is defined by an all atom rmsd structure alignment. The differences in the respective orientations are quantified by the generalized $\beta$ angle.

Table S-90: Generalized angle $\beta$ enclosed by the orientations of strychnine $\mathbf{3}$ obtained by TITANIA in the last optimization iteration of the runs $\mathbf{3}-\mathbf{A}$ (see table S-89) and the orientations of RDC@HотFCHT (see table S-88). ${ }^{[9]}$

\begin{tabular}{l|r} 
& \multicolumn{1}{|c}{$\boldsymbol{\beta}{ }^{\circ}$} \\
Set & 3-A \\
\hline set 1 & 12.852 \\
set 2 & 3.791 \\
set 3 & 9.654 \\
set 4 & 7.449 \\
set 5 & 12.323 \\
set 6 & 8.967 \\
set 7 & 18.427 \\
set 8 & 11.460 \\
set 9 & 8.322 \\
set 10 & 13.211 \\
set 11 & 11.352
\end{tabular}

The $\beta$ angles show rather low differences in between the reference and final structure of TITANIA. The main reason for deviations can be found in the strychnine structures shown in the main text figure 14. Here the plane of the aromatic ring is tilted. This leads to the differences in the calculated alignment tensors.

\subsection{RDC list}

The RDC list of the strychnine $\mathbf{3}$ run are listed in table S-91. 
Table S-91: List of all RDCs used in the runs of $\mathbf{3 - A}$.

\begin{tabular}{ll} 
RDC pair & RDC pair \\
C1-H1 & C2-H2 \\
C3-H3 & C4-H4 \\
H1-H2 & H2-H3 \\
H3-H4 & C8-H8 \\
C11-H11a & C11-H11b \\
H11a-H11b & C12-H12 \\
H11a-H12 & H11b-H12 \\
C13-H13 & H12-H13 \\
C14-H14 & H13-H14 \\
C15-H15a & C15-H15b \\
H15a-H15b & H14-H15a \\
\hline H14-H15b & C16-H16 \\
\hline H15a-H16 & H15b-H16 \\
C17-H17a & C17-H17b \\
\hline H17a-H17b & C18-H18a \\
\hline C18-H18b & H18a-H18b \\
\hline H17a-H18a & H17b-H18a \\
\hline H17b-H18b & C20-H20a \\
\hline C20-H20b & H20a-H20b \\
\hline C22-H22 & C23-H23a \\
\hline C23-H23b & H23a-H23b \\
\hline H22-H23a & \\
\hline
\end{tabular}




\section{Content of Supplementary Material}

The supplementary material is available as a zip-archive which contains all information used to perform the TITANIA runs with the respective outputs. A README.md file was added to the archive using the markdown syntax. This is also added here:

\# TITANIA: Model Free Interpretation of Residual

Dipolar Couplings in the context of Organic Compounds - Supplementary Material

\#\# Felix A. Roth, Volker Schmidts and

Christina M. Thiele

\#\# Top Level Directories

- 1-A_IPC_20_sets

- 1-B_IPC_6_indep_sets

- 1-C_IPC_6_dep_sets

- 1-D_IPC_20_sets_err

- 1-E_IPC_6_indep_sets_err

- 1-F_IPC_6_dep_sets_err

All directories listed above (IPC setups) contain individual subdirectories for the individual runs:

- ipc_xx_rdcs

- yy_ipc_xx_rdcs

where $\mathrm{xx}$ is the number of $\operatorname{RDCs}(11,17,23,31$ and 39) and yy are are variations of the runs with different settings (random [coordinates] for the input structure and non_redundant for the structure generation algorithm).

The individual subdirectories contain the input files (input.tna), output files (input.tna.out), files for alignment media specific information (input.tna.out.medium_label.ali), the trajectory file (input.tna.out.trj) and the Cartesian coordinates of every iteration step (input.tna.out.xyz). Additional directories for tubocurarine and strychnine directly contain the respective files mentioned above. These directories are:

- 2-A_tubocurarine_8_sets

- 2-B_tubocurarine_8_sets_err

- 3-A_strychnine_11_sets 
Some individual runs use different keywords, RDC data and structures. These are located in the remaining directories:

- keywords

- rdes

- structures

For more information on the syntax of the input and output files see Supporting Information section 1.2 


\section{References}

[1] T. A. Halgren, Journal of Computational Chemistry 1996, 17, 490519.

[2] J. R. Tolman, Journal of the American Chemical Society 2002, 124, 12020-12030.

[3] V. Bakken, T. Helgaker, Journal of Chemical Physics 2002, 117, 91609174 .

[4] C. Peng, P. Y. Ayala, H. B. Schlegel, M. J. Frisch, Journal of Computational Chemistry 1996, 17, 49-56.

[5] K. V. Mardia in Statistics of Directional Data, (Ed.: K. V. Mardia), Probability and Mathematical Statistics: A Series of Monographs and Textbooks, Academic Press, 1972, pp. 18-38.

[6] S. Hansmann, T. Larem née Montag, C. M. Thiele, European Journal of Organic Chemistry 2016, 2016, 1324-1329.

[7] M. Schwab, D. Herold, C. M. Thiele, Chemistry - A European Journal 2017, 23, 14576-14584.

[8] A. Marx, V. Schmidts, C. M. Thiele, Magnetic Resonance in Chemistry 2009, 47, 734-740.

[9] R. Berger, C. Fischer, M. Klessinger, Journal of Physical Chemistry A 1998, 102, 7157-7167.

[10] J.-C. Hus, R. Brüschweiler, Journal of Biomolecular NMR 2002, 24, $123-132$. 